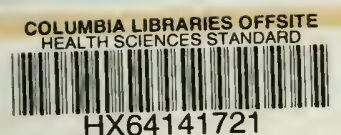

QP519.N85

Laboratory work in $p$

RECAP 


\section{QP519 N85}

(Cndumbia Entuersity in the Citu of a

College of

Zlibrary
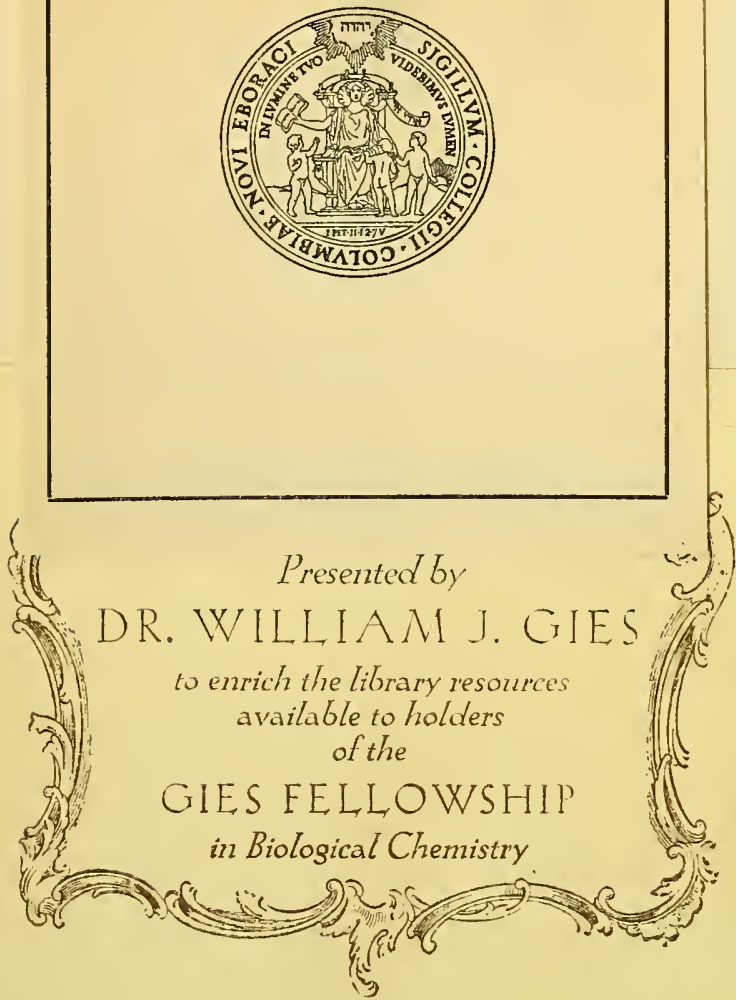

Digitized by the Internet Archive in 2010 with funding from Columbia University Libraries 



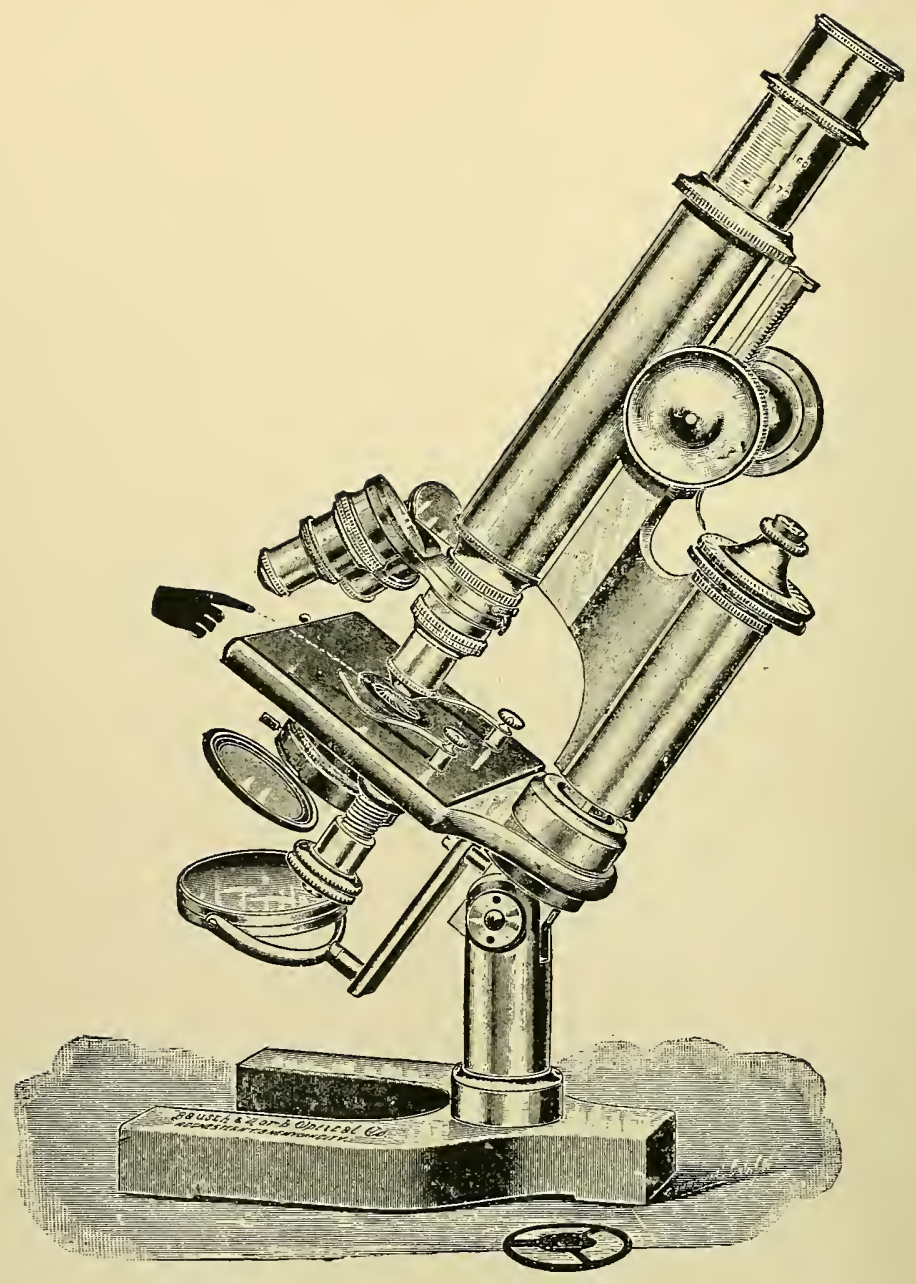




\title{
LABORATORY WORK
}

IN

\section{PHYSIOLOGICAL CHEMISTRY.}

\author{
BY \\ FREDERICK G. NOVY, Sc.D., M.D., \\ JUNIOR PROFESSOR OF HYGIENE AND PHYSIOLOGICAL CHEMISTRY, \\ UNIVERSITY OF MICHIGAN.
}

SECOND EDITION REVISED AND ENLARGED.

WITH FRONTISPIECE AND TWENTY-FOUR ILLUSTRATIONS.

ANN ARBOR :

George Wahr, Publisher, 1898 . 
Copyright, I 898 , by F. G. Novy.

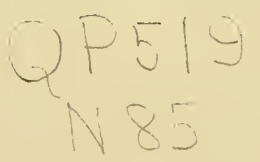

The Inland Press, Ann Arbor, Mich, 


\section{PREFACE.}

This edition is greatly enlarged and indeed wholly re-written. The directions for laboratory work cover the three great food-stuffs, and the fluids and secretions of the body. Brief explanatory descriptions of the various substances and secretions studied are added in order to give the student a better survey of the subject matter as a whole.

Every medical student should receive thorough drill in the laboratory, not merely in so-called urine analysis, but in the broader field of physiological chemistry. He should be taught to observe and to reason; to correlate the facts brought ont in the laboratory in their relation to physiology, hygiene and disease. These notes have been prepared with the object of furthering such study, and it is hoped that they will prove as useful to others as to the author's own classes in the University of Michigan. The experimental work laid down in the following pagres has been repeatedly verified in actual student work and the directions given are such as to insure results. The author's classes devote the entire afternoon, daily, for three months to this work. Even with this amount of time at their disposal certain parts of physiological chemistry must be left untouched.

The works of Salkowski, Hammarsten, v. Noorden and others bave been freely drawn upon for suggestions in the 
preparation of these directions. Thanks are due to the Bausch and Lomb Optical Co., of Rochester, for kindly supplying several cuts. I wish also to acknowledge the invaluable help rendered me by way of suggestions, verification of experimental details, and proof-reading by my able assistant, Mr. C. L. Bliss.

F. G. NOVY.

AnN ArBor, Mich., August 1, 1898. 


\section{CONTENTS.}

CHAPTER I. TAGE

FATS

7

CHAPTER IT.

CARBOHYDRATES

CHAPTER III.

Proteins

CHAPTER IV.

Saliva

CHAPTER $T$,

GASTRIC JUICE

CHAPTER VI.

PANCREATIC SECRETION

CHAPTER TII.

BILE

CHAPTER VIII,

BI,OOD

CHAPTER IX.

MILK.

CHAP'TEL $\mathrm{X}$. 
CONTENTS.

CHAPTER XI.

QUANTITATIVE ANALYSIS-

Urine, Milk, Gastric Juice, Blood . . . . . 217

CHAPTER XII.

TABLES FOR EXAMINATION OF URINE . . . . . . . 283

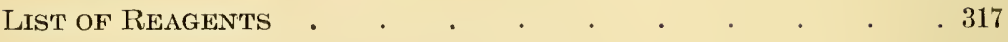

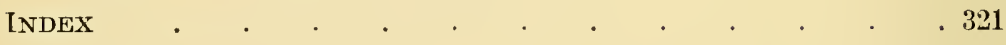




\section{CHAPTER I.}

FATS.

Fats are widely distributed in the animal and in the vegetable kingdom. Indeed it may be said that protoplasm always contains some fat, and every cell, therefore, has more or less of these compounds. In animals the fat is usually stored up in the subcutaneous tissue, or about the abdominal organs. In plants the seed, or fruit, or even the roots are rich in fats. Many bacteria contain an appreciable amount of fat stored up within their walls. This is especially true of the tubercle bacillus, which in the dry state may contain as much as 40 per cent. of fat. Its characteristic behavior to stains is probably due to this large amount of fat.

Fats are neutral compounds, or esters, resulting from the union of glycerin, $\mathrm{CH}_{2} \mathrm{OH}$. $\mathrm{CHOH} . \mathrm{CH}_{2} \mathrm{OH}$, a triatomic alcohol, with a mono-basic fatty acid. The hydrogen of each of the hydroxyl groups in glycerin is replaced by a fatty acid radical. If the fatty acid is stearic acid the resulting fat is tri-stearin, or stearin. Similarly with palmitic and oleic acids, we have the corresponding fats palmitin and olein.

Ordinary fats are really mixtures of several fats. Thus the animal fats are mixtures of variable amounts of stearin, palmitin and olein. Stearin has the higher melting point and when present in appreciable quantity it makes a relatively hard fat as tallow, or suet. On the other hand, olein is a liquid fat at ordinary temperature and mixtures of this with palmitin yield a soft fat as lard. Olive oil and other vegetable oils can be considered as nearly pure olein, although glycerides of various other fatty acids may be 
present. Milk-fat or butter consists of the three glycerides mentioned above and small quantities of glycerides of butyric and other fatty acids. The butyric acid and lower fatty acids are soluble in water and volatile, and constitute about six per cent. of the butter fat. Inasmuch as lard and tallow practically have no volatile fatty acids, this fact is utilized in the analysis of butter for adulteration.

Fats when pure are colorless, or nearly so; tasteless and odorless, and are insoluble in and lighter than water. They are soluble in boiling absolute alcohol but recrystallize on cooling. They are readily soluble in ether, chloroform, and benzol. They are emulsified or brought into a fine state of division by soap, gum-arabic, and by albuminous bodies.

Furthermore, fats when pure are neutral in reaction. As the result of exposure to physical, chemical and living agents they are readily split into their component parts, that is glycerin and fatty acids. The change that takes place is one of hydration, and whenever an ester of any kind is thus decomposed it is said to undergo saponification. This change for stearin is indicated by the following equation:-

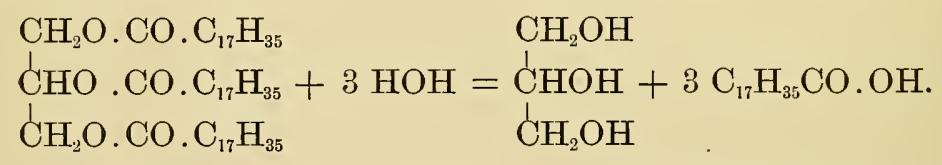

This hydration of a neutral fat can be brought about by steam under pressure; by boiling with water containing a small amount of an acid, as sulphuric acid (manufacture of glycerin, and of stearic acid or "commercial stearin" used in making of candles); by boiling with an alkali, as potash, soda, lime or lead oxide (manufacture of soap, lead plaster); by the action of air and light (rancidity of fats); by the action of bacteria; and by the action of enzymes, as that contained in the pancreatic secretion. 
The fat taken in as food is not absorbed until it reaches the intestines, where it is acted upon by the" pancreatic juice. Owing to the action of the fat-splitting ferment contained in this secretion, a portion of the fat undergoes cleavage into glycerin and fatty acid. The latter combines with the sodium carbonate present to form a sodium soap. A small amount of this soap emulsifies a large amount of fat, the fat is thus divided into very minute globules and in this form it becomes absorbed. Many bacteria exercise a similar fat-splitting action and hence fatty acids may be formed in the intestines by their activity. The bile secretion assists in the absorption of fat, and when bile cannot pass into the intestines a large amount of fat, chiefly as fatty acids, is excreted with the feces. The cells in the intestinal wall seem to have power of synthesizing fats, out of glycerin and fatty acids. This action is analogous to the synthesis by these same cells of pepton to albumin.

The fat deposited in the body is derived in part directly from the fat present in the food and absorbed as such. In addition to this some fat is made in the body out of the protein and carbohydrate molecules. The formation of fat out of albuminous material is seen in the fatty degeneration of various organs and tissues in disease. Moreover fatty acids can be obtained from albuminous substances by cleavage in the laboratory, and by bacterial decomposition. The mass of fat, or rather of fatty acids and soaps, known as adipocire and found in decomposing cadavers, probably owes its origin to the latter form of decomposition.

The fat deposited in the tissues serves as a reserve food to generate heat and energy. The large amount of carbon and hydrogen contained in the fat molecule explains the large amount of heat generated when fat is oxidized and the need of much fat as food in a cold climate. Fat, moreover, is a non-conductor of heat and thus serves to prevent undue loss of heat from the body. In starvation fat disappears rapidly from the tissues. 
The chyle and lymph may contain from 0.5 to 5 per cent. of fat. In certain diseases, as in fevers, tuberculosis, etc., the amount of fat present in the blood is increased. The blood, lymph and transsudates contain an appreciable quantity of fatty acids as a soap. Stearic and palmitic acids are met with as needle-shaped crystals in decomposing pus, in sputum from gangrene of the lung, and from tuberculosis. For the occurrence of fats and fatty acids in urine, see that chapter.

A mixture of stearic and palmitic acids is commonly called margaric acid, and a similar mixture of stearin and palmitin is designated as margarine. Oleo-margarine, a substitute for butter, is essentially purified animal fat.

1.-Preparation of Pure Fat.-For this experiment the students will use, alternately, pork-fat and suet. Cut up $10 \mathrm{~g}$. of subcutaneous pork fat, or of suet, into as small pieces as possible. Place

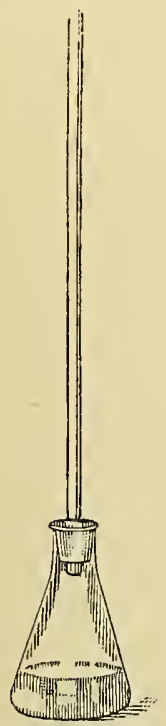

Fig, 1. into a small evaporating dish, $2 \frac{1}{2}-3$ inches in diameter, and cautiously heat over a small flame, stirring continually with a thermometer. Keep the temperature at $120^{\circ}-130^{\circ} *$ for about 10 minutes. Then strain through a small piece of muslin and squeeze thoroughly, receiving the clear fat into an evaporating dish (4 inch). Transfer the residue to a small mortar, add about 5 c.c. of strong alcohol and rub up to a fine powder. Transfer the suspension to a 75 c.c. Erlenmeyer flask, and rinse out the mortar with several successive small portions of alcohol. Insert into the neck of the flask a stopper provided with a glass tube (Fig. 1) about 24 inches long (condensing tube). Heat on the water-bath to boiling for 10 minutes. Then set aside and, when the suspended particles have settled, decant the clear alcohol onto a small filter and receive the alcoholic filtrate in the evaporating dish containing the bulk of the fat. To the insoluble residue remaining in the flask, add 20 c.c. of ether; insert the condensing tube, and cautiously boil on the water bath for about 5 minutes. Then transfer the entire contents of the flask to the filter previously used and receive

* The temperature figures given in this work are Centigrade. Unless otherwise indicated, distilled water is always to be used for dilution and solution. The reactions are to be carried out in a test-tabe unless specified otherwise. 
the ethereal filtrate in the evaporating dish. Finally wash the residue with a little ether, close the filter and squeeze out most of the ether. Then spread the filter and allow the remaining ether to evaporate spontaneously. Save this yellowish residue of connective tissue for subsequent experiments. (Chapter III, 1, 2, 3).

The evaporating dish now contains the strained fat, also the alcoholic and ethereal filtrates. Place it on a water-bath and heat carefully till all the alcohol, ether and water have been driven off and only the pure fat remains.

2.-Place a little of the pure fat, obtained as above, on a slide, corer and examine under the microscope. Observe that the little round bodies are composed of minute crystals. These are more distinct in beef fat.

3.- Transfer a piece of fat, size of a pea, by means of a glass rod to a test-tube. Add 5 c.c. of a mixture of equal parts of alcohol and ether and warm gently till dissolved. Then set aside for an hour or more and when a deposit forms transfer some of it by means of a pipette to a glass slide, cover and examine under the microscope. Sketch the crystals obtained thus from tallow and from lard. Which of these two fats crystallizes more rapidly? Why?

4.- Transfer a piece of fat to a test-tube, add 5 c.c. of alcohol and heat till dissolved. Then introduce a strip of blue litmus paper, or add a drop of an aqueous solution of litmus. Better still, to some of the alcoholic solution add a drop of alcoholic rosolic acid; a yellowish color indicates an acid reaction. What is the reaction of normal fat? Why do fats become rancid on standing for some time?

5.-Place a small piece of fat on a filter paper and warm gently over a flame, or on a heated plate till the fat melts and is absorbed. Note the transparent condition of the paper. Other substances such as glycerin, paraffin, which fill the pores of the paper have a similar action.

6.-Rub up thoroughly in a mortar a piece of fat with some $\mathrm{KHSO}_{4}$. Transfer the mixture to a $d r y$ test-tube and heat cautiously. The peculiar irritating odor or sensation is due to acrolein or acrylic aldehyde, which is formed by dehydration from the glycerin of the fat.

Glycerin, $\mathrm{CH}_{2} \mathrm{OH}$. $\mathrm{CHOH} . \mathrm{CH}_{2} \mathrm{OH}$.

Acrolein, $\mathrm{CH}_{2}$ : $\mathrm{CH}$. $\mathrm{COH}$.

7.-To a small piece of fat in a test-tube add about 10 c.c. of a semi-saturated solution of sodium carbonate, warm and shake thoroughly. The liquid becomes milky, but on standing, most of the fat 
collects on the surface. The liquid below shows but slight cloudiness. Neither emulsion, solution, or saponification has taken place.

8.-Saponification.-Melt the fat that is left from the preceding experiments and transfer it to a 150 c.c. Erlenmeyer flask. Then add 20-30 c.c. of alcohol and $3 \mathrm{~g}$. of $\mathrm{KOH}$. Insert a condensing tube and heat on the water-bath for about a half an hour. Saponification takes place rapidly. To ascertain if the change is complete pour a little of the alcoholic fluid into a few c.c. of water. The liquid must remain clear. If it becomes cloudy it is due to oil drops and shows that the saponification is incomplete. The solution eventually contains soap, glycerin, and excess of alkali and alcohol.

9. - Separation of the Fatty Acids. - To about 100 c.c. of water in a small beaker add 3 c.c. of $\mathrm{H}_{2} \mathrm{SO}_{4}$. Then warm to about $50^{\circ}$. Pour the soap solution, gradually, and with constant stirring, into the warm acid liquid. The fatty acids are set free and rise to the surface, forming a clear, oily liquid. Place the beaker on a water-bath and heat till the aqueous liquid below the fatty acid layer becomes almost clear, and all the fatty acid has risen to the surface. At the same time prepare about 400 c.c. of boiling water.

Then transfer the contents of the beaker to a small filter, previously moistened with hot water. The fatty acids, while still liquid, are washed on the filter with hot water (10-12 times) till the wash water ceases to give with $\mathrm{BaCl}_{2}$ a test for $\mathrm{H}_{2} \mathrm{SO}_{4}$. Collect the aqueous filtrate and wash-water and set it aside to be examined later for glycerin.

The funnel containing the washed fatty acids is now placed upright in a small beaker containing cold water, the level of which should correspond to that of the fatty acid on the filter. The fatty acids solidify. The product thus obtained is a mixture of oleic acid, $\mathrm{C}_{18} \mathrm{H}_{34} \mathrm{O}_{2}$, palmitic acid $\mathrm{C}_{16} \mathrm{H}_{32} \mathrm{O}_{2}$, and stearic acid $\mathrm{C}_{18} \mathrm{H}_{36} \mathrm{O}_{2}$. Commercial stearin which is used in the manufacture of candles is a mixture of palmitic and stearic acids.

Butter fat when treated as above yields fatty acids, a part of which ( 7 per cent, or more) are soluble in hot water; the remainder (not over 88 per cent), consists of insoluble fatty acids and remains on the filter. All other animal fats, as lard, tallow, etc., yield 95.5 per cent. of insoluble fatty acids (Hehner and Angell's method for analysis of butter).

\section{Reactions with Fatty Acids.}

10. - To some of the solid fatty acid in a test-tube add about 10 c.c. of strong alcohol and warm till the acid dissolves. Divide into 
three portions. To one portion add a drop of rosolic acid; to another portion 1-2 drops of aqueous litmus solution; to a third portion a strip of blue litmus paper. What is the reaction, and which reagent is most delicate?

11.- To a portion of the fatty acid apply the test given under Exp. 5.

12.-To a portion of the fatty acid apply the test given under Exp. 6. What is the result?

13. - To about 10 c.c. of a semi-saturated $\mathrm{Na}_{2} \mathrm{CO}_{3}$ solution add a small portion of the fatty acids and heat. An effervescence results, carbonic acid is given off. The fatty acids dissolve and a sodium soap is formed. Place the tube in a beaker of cold water, a soap jelly results.

Warm the tube again till the contents are liquid, then add $1-2$ drops of cotton-seed or olive oil and shake. An opalescent liquid, or emulsion forms. Transfer a drop of this to a slide, cover and examine under the microscope. Note the highly refracting fat globules. Soap solutions emulsify neutral fats. Importance of this fact in the physiological absorption of fat.

14.-Place some of the fatty acid in a small beaker, add about 50 c.c. of water and warm gently till the fatty acids melt. Then add dilute $\mathrm{NaOH}$, drop by drop, and stir thoroughly after each addition. Continue addition of alkali till the fatty acid just dissolves. With this sodium soap solution make the following tests:

(a). To some of the solution add a few drops of $\mathrm{CaCl}_{2}$ solution An insoluble calcium stearate, etc., forms. This calcium soap is formed when hard water is used with soap. What is meant by hardwater?

(b). To another portion add some lead acetate and warm gently. The white sticky precipitate which forms is lead soap. It is known and used medicinally as lead plaster. Oleate of lead is soluble in ether-distinction from palmitin and stearin.

15. - Separation of Glycerin, $\mathrm{C}_{3} \mathrm{H}_{5}(\mathrm{OH})_{3}$. - The combined aqueous filtrate and washings from the fatty acids (Exp. 9), should, if oily globules are present, be filtered through a wet filter. The filtrate is then carefully neutralized with $\mathrm{NaOH}$ and concentrated in an evaporating dish, first over a flame, and finally on a water-bath almost to dryness. To the residue add about 25 c.c. of alcohol, stir thoroughly and allow the mixture to stand for $1 / 4-1 / 2$ hour, then filter. To the residue add another portion of about 15 c.c. of alcohol, stir well and transfer this washing to the filter. Evaporate the alcoholic filtrate and washings, 
on the water-bath, to dryness. Take up the residue with about 15 c.c. of absolute alcohol and transfer this entire mixture to a large testtube; then add an equal volume of ether, cork and shake, and set aside in a beaker of cold water for about a half an hour. Filter off the salts which are thus thrown out of solution and cautiously evaporate the alcoholic-ethereal liquid on a slightly warmed water-bath. A syrupy residue remains,-glycerin.

16. - Taste the yellowish syrup that is left.

17.-Place a drop of the residue on a slide and add a little powdered borax. Then touch the mixture with a platinum wire and place this in a Bunsen flame. Note the green color.

18.-Mix a drop or two of the syrup with some powdered $\mathrm{KHSO}_{4}$ and heat in a dry test-tube. Compare the sesult with that obtained in experiments 6 and 12.

19. - To some glycerin solution add a little sodium hydrate then a few drops of copper sulphate. Instead of a precipitate a blue solution results.

Glycerin on treatment with a mixture of sulphuric and nitric acids yields an ester, $\mathrm{C}_{3} \mathrm{H}_{5}\left(\mathrm{O} . \mathrm{NO}_{2}\right)_{3}$, commonly known as nitro-glycerin. When mixed with sand or infusorial earth it is known as dynamite. 


\section{CHAPTER II.}

\section{CARBOHYDRATES.}

In this group are usually placed those substances which contain $\mathrm{H}$ and $\mathrm{O}$ in the same proportion as does water $(2: 1)$ and 6 carbon atoms or a multiple of 6 . Recent investigations have shown that we may have carbohydrates containing from 4 to 9 or more carbon atoms. There are, furthermore, unquestionable sugars, such as rhamnose, which do not have $\mathrm{H}$ and $\mathrm{O}$ in the proportion of 2 to 1 .

Carbohydrates are present in comparatively small amounts in the animal body, either free or as constituents of certain complex proteids. They constitute, however, the greater part of the solids of plants, just as proteids make up the greater part of the solids of the animal body. They are aldehyde or ketone derivatives of certain alcohols.

The following condensed classification is adapted from Tollens:

\section{Mono-Saccharides or Glycoses.}

This includes besides others, pentoses, $\mathrm{C}_{5} \mathrm{H}_{10} \mathrm{O}_{5}$, and hexoses, $\mathrm{C}_{6} \mathrm{H}_{12} \mathrm{O}_{6}$, such as dextrose, laevulose; and rhamnose, $\mathrm{C}_{6} \mathrm{H}_{12} \mathrm{O}_{5}$.

II. Di-Saccharides, or Saccharoses, $\mathrm{C}_{12} \mathrm{H}_{22} \mathrm{O}_{11}$.

Cane-sugar, milk-sugar, maltose, iso-maltose.

\section{Poly-Saccharides.}

A few of these compounds are crystallizable, but most of them are amorphous. The latter group includes pentosanes, which have the same relation to pentoses as starch bears to glucose. Also, starch, and its derivatives amylodextrin, erythrodextrin, and achroodextrin. Also glycogen, dextran, and others; cellulose.

IV. Mannite, $\mathrm{C}_{6} \mathrm{H}_{14} \mathrm{O}_{6}$.

The compounds of this group are related to the true carbohydrates. 


\section{Inosite, $\mathrm{C}_{6} \mathrm{H}_{12} \mathrm{O}_{6}$.}

This is a derivative of hexamethylene $\mathrm{C}_{6} \mathrm{H}_{12}$.

As shown from the following formulæ, dextrose or glucose contains an aldehyde group, whereas laevulose or fructose contains a ketone group:

Dextrose $=\mathrm{CH}_{2} \mathrm{OH} . \mathrm{CHOH} . \mathrm{CHOH} . \mathrm{CHOH} . \mathrm{CHOH} . \mathrm{CHO}$.

Laevulose $=\mathrm{CH}_{2} \mathrm{OH} . \mathrm{CHOH} \cdot \mathrm{CHOH} . \mathrm{CHOH} \cdot \mathrm{CO} \cdot \mathrm{CH}_{2} \mathrm{OH}$.

On treatment with nascent hydrogen the aldehyde or ketone group is readily reduced to the corresponding alcohol group $\mathrm{CH}_{2} \mathrm{OH}$, or $\mathrm{CHOH}$ yielding mannite $\mathrm{C}_{6} \mathrm{H}_{14} \mathrm{O}_{6}$. The pentoses in a similar way yield corresponding pentites.

The mono-saccharides, like aldedydes, readily reduce salts of silver, copper, mercury, etc. It should be remembered that these salts are also reduced by other substances, as lactose, maltose, glucuronic acid, "alkapton."

\section{PENTOSES, $\mathrm{C}_{5} \mathrm{H}_{10} \mathrm{O}_{5}$.}

In plants there are substances, pentosanes, which yield on hydration pentoses just as starch on similar treatment yields glucose. A pentose has been met with in the decomposition of a glyco-proteid obtained from the pancreas. It has been found recently in several urines; also in the urine of ordinary and of experimental diabetes.

The pentoses are strong reducing agents, but are not fermentable by yeasts. With phenyl-hydrazin they yield osazons which melt at $157^{\circ}-160^{\circ}$. On distillation with hydrochloric acid they yield furfurol, which colors anilin acetate paper bright red.

\section{HEXOSES, $\mathrm{C}_{6} \mathrm{H}_{12} \mathrm{O}_{6}$.}

Cane-sugar, $\mathrm{C}_{12} \mathrm{H}_{22} \mathrm{O}_{11}$, which yields dextrose and laevulose, $\mathrm{C}_{6} \mathrm{H}_{12} \mathrm{O}_{6}$, on hydration, can therefore be considered as an anhydride of these hexoses. The hexoses, dextrose and laevulose, are widely distributed in plants, especially in acid fruits; and furthermore, readily form on hydration of 
starch, cane-sugar, glucosides as phloridzin, etc. Another hexose, galactose, results on hydration of lactose, and of other carbohydrates, and also of cerebrin.

The three hexoses mentioned are fermentable by yeast. On heating with dilute mineral acids they yield laevulinic acid, $\mathrm{C}_{5} \mathrm{H}_{3} \mathrm{O}_{3}$, and humous substances.

Dextrose or glucose, also known as grape-sugar or starchsugar, is formed during digestion. It is present in small amount, $0.1-0.2$ per cent., in the blood; in still less amount in normal urine. In diabetes it is present sometimes in considerable quantities as the characteristic constituent of urine. After the digestion of large quantities of canesugar, lactose or glucose, a reducing substance appears in the urine (alimentary glycosuria). A part of the canesugar may appear in the urine as such. Glucose appears in the urine after administration of phloridzin, uranium salts, hydrocyanic acid; also when the oxygen supply is diminished, and hence in CO poisoning. Reducing substances, presumably glucose, are formed in the decomposition of cartilage, nucleinic acid, paranuclein, nucleoproteid of the pancreas, etc.

It can be obtained as minute crystals which are either anhydrous or contain one molecule of water. It is only about $3-5$ as sweet as cane-sugar. It is soluble in about an equal part of water; insoluble in absolute alcohol. The solutions are dextro-rotatory. The melting-point is at $144^{\circ}-146^{\circ}$; above $200^{\circ}$ caramel forms.

\section{Glucose.}

In the following experiments, unless otherwise indicated, a two per cent. solution of glucose is employed:

1.-Molisch's Reaction.-To about $1 / 2$ c.c. of the dilute sugar solution add 1-2 drops of an alcoholic solution (15 per cent.) of $a$-naphthol. Then add slowly about 1 c.c. of concentrated ${ }_{1} \mathrm{H}_{2} \mathrm{SO}_{4}$ so that it runs down the side of the inclined tube and forms a laver. A beautiful reddish violet ring forms at the zone of contact. 
This is a general reaction due to the formation of furfurol and is given by all carbohydrates. Apply this test to some normal urine; and to urine diluted with 5 parts of water. If the test is given by the latter it indicates that the carbohydrates of the urine are increased.

2.-Place some of the dry glucose in a tube and heat gently over a flame. It melts, then turns yellow and finally dark-brown. The peculiar odor is that of burnt-sugar. Allow the tube to cool, then add water and warm slightly. Note the dark-yellow or brownish color of the solution. Caramel is a harmless coloring matter and is employed extensively for coloring liquors, vinegars, etc.

3.- To some dry glucose add cold, concentrated $\mathrm{H}_{2} \mathrm{SO}_{4}$ and let stand. The liquid remains colorless, or at most is light yellow. Distinction from cane-sugar. See experiment 3 under cane-sugar. After comparing this with the corresponding experiment with cane-sugar, gently heat the glucose tube. It promptly blackens, due to humin substances. Laevulinic acid is formed at the same time.

4.- To the sugar golution add some strong $\mathrm{KOH}$ solution and heat. The liquid becomes yellow, then dark-brown. The sugar readily undergoes oxidation in alkaline solution. With solid $\mathrm{KOH}$, because of the heat generated, the reaction is sometimes violent.

This test when applied to urine is known as MooreHeller's test. In that case the precipitate that forms is due to earthy phosphates. The test is not particularly delicate and is certainly not reliable since other substances may yield dark solutions under similar conditions, namely, alkapton, lactose, maltose, etc.

Compare this reaction with experiment 4 under canesugar.

5. - To the sugar solution add $1 / 2$ volume of $\mathrm{Na}_{2} \mathrm{CO}_{3}$ solution, then 1-2 drops of a freshly prepared solution of potassium ferricyanide, and boil. The liquid becomes colorless-due to a reduction of the salt to a ferrocyanide.

6. - To the sugar solution add a little ammoniacal silver nitrate and a few drops of $\mathrm{KOH}$ and warm gently. A mirror of metallic sil- 
ver forms, especially if the solutions are dilute. The silver has been reduced.

The ammoniacal silver nitrate is prepared by adding ammonium hydrate to silver nitrate till the precipitate just disappears.

7.-To the sugar solution add one drop of a freshly prepared solution of sodium indigo sulphate, also add a little $\mathrm{Na}_{2} \mathrm{CO}_{3}$ solution and heat. The blue color changes first to violet, then to red, yellow, and finally the liquid is colorless. The indigo has been reduced to indigo-white. Cool the tube under the hydrant and shake. The indigo-white is oxidized to indigo-blue. On heating the blue is again reduced. Litmus and other coloring agents are reduced in a similar manner.

The following reactions should be applied, side by side, to the aqueous solution of glucose and to diabetic urine, or to urine containing about one per cent. of sugar.

8.-Trommer's Test.--Render the solution or urine strongly alkaline with $\mathrm{KOH}$ and boil, then add a few drops of copper sulphate solution and warm again: a reddish-yellow precipitate of cuprous oxide forms. If excess of copper be added, the copper hydrate precipitate will mask small amounts of the red precipitate. If too little copper has been added, a white precipitate of uric acid and nuclein bases (alloxuric bodies) may form.

8r.-Fehling's Test.-Boil some Fehling's solution in a test-tube and then add the sugar solution, or the suspected urine, and boil. Cuprous oxide is thrown down.

The urine if strongly acid should be rendered alkaline. This is the test commonly employed when examining urine for sugar. It should be remembered that it is not an absolute test, since the urine, in rare cases, may contain other reducing substances (alkapton). A small amount of sugar may, moreover, escape detection, since the cuprous oxide may be held in solution by creatinin and other urine constituents. 
It should furthermore be remembered that Fehling's solution deteriorates on keeping, so that on heating the solution itself, a red precipitate of cuprous oxide may form. It is advisable, therefore, to keep the two constituents of Fehling's solution in separate bottles and to mix equal volumes just before use.

Pavy's solution, employed for the same purpose, is a solution of copper hydrate in ammonium chloride.

9.-To some Barfoed's solution add some glucose solution and boil. The cuprous oxide precipitate forms. Milk-sugar, cane-sugar, maltose and dextrin do not reduce this solution.

Barfoed's reagent is an acid solution of copper. It is prepared by dissolving one part of copper acetate in 15 parts of water. To 200 c.c. of this solution, 5 c.c. of a 38 per cent. acetic acid solution are added.

10.-Boettger's Test.--Render the specimen alkaline with sodium or potassium hydrate, then add a minute quantity of basic bismuth nitrate and heat-a black color or precipitate, due to reduced bismuth, forms. Albumin if present must be removed. Owing to the action of the alkali on the sugar the solution may color. This can be avoided by substituting sodium carbonate for the alkali.

10a.-Nylander's Test.-Dissolve $10.33 \mathrm{~g}$. of 'sodium hydrate in 100 c.c. of water; add $2 \mathrm{~g}$. of basic bismuth nitrate, and $4 \mathrm{~g}$. Rochelle salts; warm and filter. This reagent keeps better than Fehling's solution.

To 10 volumes of the sugar solution or urine add one volume of the reagent and boil $2-3$ minutes. Then let stand for $10-15$ minutes.

Concentrated urines may become blackish with this reagent; this may also occur if chrysophanic acid is present in the urine. On the other hand the reaction is more delicate than Fehling's solution, where, as pointed out, small amounts of cuprous oxide may be held in solution.

Alkaline solutions of mercury salts are also employed in testing for glucose (Knapp, Sachsse). 
11. - Phenyl-hydrazin Test.-Phenyl-hydrazin on heating with sugar forms phenyl-glucosazon, $\mathrm{C}_{6} \mathrm{H}_{10} \mathrm{O}_{4}\left(\mathrm{~N}_{2} \mathrm{H} . \mathrm{C}_{6} \mathrm{H}_{5}\right)_{2}$. This forms bundles of yellow needles which melt at $204-205^{\circ}$.

$$
\mathrm{C}_{6} \mathrm{H}_{12} \mathrm{O}_{6}+2 \mathrm{C}_{6} \mathrm{H}_{5} \cdot \mathrm{N}_{2} \mathrm{H}_{3}=\mathrm{C}_{18} \mathrm{H}_{22} \mathrm{~N}_{4} \mathrm{O}_{4}+2 \mathrm{H}_{2} \mathrm{O}+2 \mathrm{H}
$$

Application to the urine.-Place in a small beaker about 50 c.c. of the clear urine add $1-2 \mathrm{~g}$. of phenyl-hydrazin hydrochloride and about $2-4 \mathrm{~g}$. of sodium acetate, cover with a watch-glass and warm on the water-bath for $1 / 2-1$ hour, then turn off the light and allow the solution to cool on the water-bath. Examine under the microscope the deposit which forms. If amorphous, or if it is desirable to purify the crystals, dissolve on the filter in hot alcohol. To the filtrate add water and boil till the alcohol is expelled-on cooling, the characteristic yellow crystals appear. Filter, wash, dry and determine the melting-point (see urea).

The phenyl-hydrazin reaction with sugars is of very great importance in their identification. It forms with sugars, when heated sufficiently long on the water-bath, osazones. The various sugars yield, therefore, correspond. ing osazones, which are yellowish, and differ in crystalline form, melting-point, solubility, and optical behavior. The determination of the melting-point is especially valuable.

12.-Fermentation Test-Rub up some of the solution or of the suspected urine with a little yeast. Fill the mixture into a large, wide test-tube provided with a perforated stopper through which passes a tube bent into a $U$ shape-the free arm being longer than the one that passes through the cork. Care should be taken to fill the tube so that no air is present in the test-tube when it is inverted. Set the tube aside in an inverted position in a warm place for 24 hours and observe the accumulation of gas-fermentation.

When the fermentation is completed place the tube in an upright position in a dish of water, remove the stopper and by means of a bent pipette introduce a little potassium hydrate solution. What is the result?

$$
\mathrm{C}_{6} \mathrm{H}_{12} \mathrm{O}_{6}=2 \mathrm{C}_{2} \mathrm{H}_{5} \mathrm{OH}+2 \mathrm{CO}_{2} \text {. }
$$

Under the influence of certain bacteria, glucose readily undergoes lactic acid, butyric acid, or viscous fermentation. 
Laevulose, also known as fruit-sugar or fructose, occurs widely distributed in the plant kingdom. It is also present with dextrose in honey. While starch on hydration yields dextrose, there are analogous substances, as inulin, $\mathrm{C}_{6} \mathrm{H}_{10} \mathrm{O}_{5}$, which on similar decomposition yield laevulose. In exceptional cases it has been met with in the urine of diabetes. When administered in diabetes a part may be changed to glucose and to glycogen, and a part may be eliminated as such (Haycraft).

This sugar crystallizes with great difficulty and for that reason it is ordinarily met with as a thin syrup. It is readily soluble in water, insoluble in cold absolute alcohol. The solutions are laevo-rotatory.

The rotation is greater, and in opposite direction, than that of cane-sugar. Hence on hydration of cane-sugar the resulting mixture is laevo-rotatory, and is therefore called invert-sugar. Inversion, as applied to complex carbohydrates, is synonymous with hydration.

Like glucose, it readily reduces metallic oxides; is fermented by yeast and forms the same osazon.

Galactose is formed with dextrose on hydration of milksugar, and other carbohydrates; also of cerebrin. In yellow lupine a compound, galactite (Ritthausen), is present which yields galactose on hydration. It crystallizes in needles or plates which melt at $168^{\circ}$. It is dextro-rotatory. It reduces Fehling's solution and is said to ferment with yeast. It forms an osazone which melts at $193^{\circ}$. On oxidation it yields mucic acid-distinction from dextrose. The origin of galactose as a constituent of milk-sugar is not known. It may be derived from antecedents in the plant food, and on the other hand may be formed from glycogen or even glucose in the body.

CANE SUGAR, $\mathrm{C}_{12} \mathrm{H}_{22} \mathrm{O}_{11}$.

Saccharose, Sucrose.-This sugar is widely distributed in 
plants in the leaves of which it is formed, under the influence of light and possibly of chlorophyll. It is then trans. ported to different parts of the plant and may be stored up in the roots as in the case of beet-root, or in the stalk, as in sugar cane. In acid liquid it very readily undergoes inversion and for that reason it is not present in strongly acid fruit juices but is represented there by dextrose and laevulose. In moderately acid fruits as nuts, apples, melons, bananas, sweet oranges, it is present as such with more or less glucose. The cane sugar which is removed from the flower by the bee becomes almost wholly inverted when made into honey.

It forms large monoclinic crystals which dissolve in 1.5 parts of water at $20^{\circ}$. The solution is strongly dextrorotatory. It melts at $160^{\circ}$ and on further heating it yields caramel. It is decomposed by dilute acids, slowly in the cold, very rapidly on heating. The change is as follows;

$$
\mathrm{C}_{12} \mathrm{H}_{22} \mathrm{O}_{11}+\mathrm{H}_{2} \mathrm{O}=\underset{\text { Dextrose. }}{\mathrm{C}_{6} \mathrm{H}_{12} \mathrm{O}_{6}}+\underset{\text { Laevulose. }}{\mathrm{C}_{6} \mathrm{H}_{12} \mathrm{O}_{6}}
$$

This hydration is also brought about by many ferments, such as the invertin of yeast; by bacteria and moulds; also by the acid gastric juice but not by the pancreas. Once inverted the resultant invert-sugar is readily subject to various fermentations such as alcoholic, viscous, lactic acid, etc.

Before inversion it is strongly dextro-rotatory and does not reduce Fehling's solution. After inversion it is less dextro-rotatory, or even laevo-rotatory, and reduces Fehling's solution. With phenyl-hydrazin it does not form a corresponding osazon, but does form, owing to inversion, the phenyl-glucosazon. This behavior and the non-reduction of metallic oxides distinguishes cane-sugar from maltose and lactose. The latter, therefore, still show the aldehyde character. 
Apply the following reactions, with the exception of 2 and 3 , to a 2 per cent. aqueous solution of cane-sugar, and compare these, side by side, with the corresponding reactions of glucose:

1.-Molisch's Reaction.-Apply as given in Exp. 1, under glucose

2.-Caramel Reaction.-Apply as given in Exp. 2, under glucose.

3.-Sulphuric Acid Reaction.-Apply as given in Exp. 3, under glucose. The cold acid in a few minutes colors yellow, then becomes black-distinction from dextrose. Humin substances are formed.

4.--Potassium Hydrate Reaction.-Apply as given in corresponding test under glucose and carefully note the difference.

5.-Apply Fehling's solution as in Exp. $8 a$ under glucose.

6.-Test with Barfoed's reagent, as in Exp. 9 for glucose.

7.-Test with Nylander's reagent, as in Exp. $10 a$ under glucose.

8.-Apply the fermentation test as in Exp. 12 under glucose, and compare the rapidity of fermentation with that of glucose.

9.-Place 50 c.c. of the cane-sugar solution in a small beaker, add 6-8 drops of concentrated $\mathrm{HCl}$ and boil for $2-3$ minutes. Then cool, render alkaline with sodium or potassium hydrate. To this solution now apply tests $4,5,6,7$, as given above. Note the results.

\section{Lactose, $\mathrm{C}_{12} \mathrm{H}_{22} \mathrm{O}_{11}+\mathrm{H}_{2} \mathrm{O}$.}

Lactose, or milk-sugar, occurs probably in the milk of all animals. The amount present varies from $3-5-6$ per cent. It has been found in the urine during the later stages of pregnancy and immediately after birth. It is said to occur in one plant.

It forms large rhombic crystals which are soluble in 6 parts of cold water and $2 \frac{1}{2}$ parts of boiling water. The solution is dextro-rotatory. When heated to $170-180^{\circ}$ it forms a lacto-caramel, $\mathrm{C}_{6} \mathrm{H}_{10} \mathrm{O}_{5}$; melts at $203.5^{\circ}$. On heating with acids, hydration takes place according to the equation:

$$
\mathrm{C}_{12} \mathrm{H}_{22} \mathrm{O}_{11}+\mathrm{H}_{2} \mathrm{O}=\underset{\text { Galactose. }}{\mathrm{C}_{6} \mathrm{H}_{12} \mathrm{O}_{6}}+\underset{\text { Dextrose. }}{\mathrm{C}_{6} \mathrm{H}_{12} \mathrm{O}_{6}} .
$$

On further heating with acids, humin and formic and laevulinic acids form. On oxidation with nitric acid, inversion first takes place as above, and then the galactose is oxi- 
dized to mucic acid, whereas the dextrose forms saccharic acid. It reduces Fehling's solution but is only $2 / 3$ as strong as dextrose. Unlike the latter, it is not fermented by yeast. Bacteria readily bring about lactic acid fermenta tion. In kephir and kumyss the sugrar is changed to alcohol and lactic acid.

With phenyl-hydrazin it combines to form a lactosazon which crystallizes on cooling as round aggregates of yellow needles which melt at $200^{\circ}$. Its behavior to cold concen trated sulphuric acid and to alkalis also serves to distinguish it from dextrose and cane-sugar respectively. Alkalis yield lactic acid and pyrocatechin.

To a two per cent. solution of lactose apply the tests $1-8$ as given under cane-sugar. For the preparation of milk-sugar see Milk. Lactose is estimated according to the method given under Milk analysis (Chapter XI).

\section{Maltose, $\mathrm{C}_{12} \mathrm{H}_{22} \mathrm{O}_{11}+\mathrm{H}_{2} \mathrm{O}$.}

This sugar is formed by the action of the ferment dias. tase, contained in malt or sprouting barley, on starch. It is also formed by the ferments of the saliva, pancreas and liver. The formation of dextrin precedes that of maltose. When starch is heated with $\mathrm{H}_{2} \mathrm{SO}_{4}$, maltose is temporarily produced. Consequently crude glucose and glucose syrup will contain maltose in small amounts.

It forms fine white needles, grouped in little masses. It is soluble in water and in dilute alcohol. On oxidation with nitric acid it yields saccharic acid. On heating with sulphuric acid it yields two molecules of dextrose. This change is also accomplished by ferments.

$$
\mathrm{C}_{12} \mathrm{H}_{22} \mathrm{O}_{11}+\mathrm{H}_{2} \mathrm{O}=\mathrm{C}_{6} \mathrm{H}_{12} \mathrm{O}_{6}+\mathrm{C}_{6} \mathrm{H}_{12} \mathrm{O}_{6} \text {. }
$$

Like dextrose it is easily fermented by yeast, and readily reacts with potassium hydrate, and with Fehling's solution. It reduces the latter more weakly than does dextrose; 10 
c.c. of Fehling's solution represents $77.8 \mathrm{mg}$. maltose. The reaction with Barfoed's reagent serves to distinguish it from dextrose. With phenyl-hydrazin it forms an osazon-maltosazon. This forms yellow separate needles, which melt at $206^{\circ}$. It dissolves, or can hold in solution, ferric hydrate. It is dextro-rotatory.

Iso-Maltose, is an isomer of maltose. It is amorphous and is formed by the action of acids and ferments on starch. It has been prepared synthetically from glucose by the action of concentrated $\mathrm{HCl}$. Unlike maltose, it is more difficultly fermentable, and forms an osazon which melts at $153^{\circ}$ It readily reduces Fehling's solution. It is converted by diastase into maltose.

The relation of the three di-saccharides can be seen from the following:

$$
\begin{aligned}
& \text { Cane-sugar }+\mathrm{H}_{2} \mathrm{O}=\text { glucose }+ \text { laevulose. } \\
& \text { Milk-sugar }+\mathrm{H}_{2} \mathrm{O}=\text { glucose }+ \text { galactose. } \\
& \text { Maltose }+\mathrm{H}_{2} \mathrm{O}=\text { glucose }+ \text { glucose. }
\end{aligned}
$$

1. - To 100 c.c. of boiling water add $10 \mathrm{~g}$. of starch and stir till an even starch paste forms. Then cool to $60^{\circ}$ and add $1 \mathrm{~g}$. of powdered malt, immerse in a water-bath at $60^{\circ}$ for one hour. At intervals of 10 minutes test $1--2$ c.c. of the liquid with iodine for dextrin (see page 29) Then boil and filter. Evaporate $1 / 2$ of the filtrate to a thick syrup and set this aside for several days to crystallize. The addition of a thread, or of a crystal of maltose will favor crystallization. Note the taste of the syrup.

To the remaining $1 / 2$ of the filtrate apply the tests $4-8$ inclusive as given under cane-sugar.

\section{Starch, $\left(\mathrm{C}_{6} \mathrm{H}_{10} \mathrm{O}_{5}\right)_{n}$.}

Starch, or amylum, is a highly complex carbohydrate and the value of $n$ in the above formula is not determined. It is placed by some at as high as 200. Starches are also known as glucosins, since on hydration they yield as a final product glucose or dextrose, whereas the inulins or laevulins, which correspond to starch, yield laevulose. The 
inulins are comparatively rare, whereas starch is a most widely distributed plant constituent. It is evidently formed from $\mathrm{CO}_{2}$ by chlorophyll in the presence of water. In plants the excess of sugar is stored up as starch, while in animals it is stored up as glycogen. In the body of animals starch can unquestionably be converted into and deposited as fat. It is known that bacteria acting on starch can give rise to certain fatty acids.

Starch is contained in the so-called starch-granules, which have a characteristic appearance and can be readily recognized under the microscope. The form of the granules as obtained from one plant differs from that obtained from other plants. The size of the granules varies greatly even in starch of the same variety. The starch proper is deposited in these granules in layers around one or more nuclei. Some cellulose is present. Frequently, as a result, concentric rings will be observed in the starch granule.

On heating to $150-170^{\circ}$ it becomes yellowish, and also soluble in water; that is, dextrin is formed. Commercial dextrin, which is used extensively as a mucilage, is prepared in this way.

Starch is insoluble in cold water. In the presence of chloride of zinc and other salts it swells up and dissolves. On heating with water to $60-70^{\circ}$ it swells to a paste but does not form a true solution. At a higher temperature it does dissolve, forming soluble starch and hydrolytic products described below. With glycerin, especially on heating, it forms soluble starch. On heating with dilute acids it dissolves readily, or is rather hydrated, forming soluble products. The final product of the action of an acid is dextrose. $\mathrm{HCl}$ acts more rapidly than $\mathrm{H}_{2} \mathrm{SO}_{4}$. Diastatic enzymes, such as are contained in malt, saliva, pancreas, dissolve starch, forming a number of intermediate products and finally maltose, not dextrose. Starch is not fermented by yeast but is affected by bacteria, such as lactic acid and butyric acid bacilli; also by moulds. Nitric acid first 
inverts starch, then oxidizes; the products are saccharic, tartaric, and oxalic acids.

The products formed by the hydration of starch, brought about by water under pressure, by acids, or by ferments, are presented in the following table:

\begin{tabular}{|c|c|c|c|c|c|}
\hline $\begin{array}{l}\text { Starch ........... } \\
\text { Soluble Starch .... }\end{array}$ & Iodine, & blue. & Fehling, & $\begin{array}{l}0 \\
0\end{array}$ & Tasteless \\
\hline Erythrodextrin . & " & red. & 66 & + very slight. & "6 \\
\hline & " & 0. & "6 & + slight. & ،6 \\
\hline Malt & 6 & 0 . & 6 & & Sweetish. \\
\hline Iso & 6 & 0. & “ & + & Sweet. \\
\hline Malto & 6 & 0. & 6 & + Barfoed, 0 & $" 6$ \\
\hline Dextrose & 6 & 0. & 6" & + & 66 \\
\hline
\end{tabular}

1.-Examine microscopically and sketch the granules of the following starches: potato, wheat, buckwheat, corn, arrowroot and rice. Note the shape of granules, the number of rings if any, and the cleft or hilum.

2.-Place a little starch in a test-tube, add water and shake thoroughly, then filter. To the filtrate add a drop of iodine solution. No color is formed, since starch is insoluble. Add a drop or two of iodine to the residue on the filter - a blue color results.

3.-Soluble Starch.--Place 100 c.c. of water in a beaker and boil, then add $\mathrm{lg}$. of powdered starch and continue boiling for $2-3$ minutes, stirring constantly. A starch paste forms.

4.--Place some of the starch solution obtained in experiment 3 in a tube and add a drop of iodine solution. A deep-blue color results Now heat the contents of the tube; the color disappears, to reappear on cooling. The blue color is due to the so-called starch iodide, which possesses a variable composition.

5.-To some of the starch solution add an excess of tannic acid. A yellowish white precipitate forms or the liquid becomes highly opaque.

6.-Boil some of the starch solution with Fehling's solution. No reaction takes place.

7.- To about 50 c.c. of the starch solution in a beaker add $1 / 2$ c.c. of $\mathrm{H}_{2} \mathrm{SO}_{4}$, cover with a watch-glass and boil for 15 minutes. Replace the water that may be lost by evaporation. Now place some of the 
liquid in a tube, render alkaline with sodium or potassium hydrate, add some Fehling's solution and boil. If no reduction takes place, continue heating the contents of the beaker for another 15 minutes and test as before. Inversion has taken place; a reducing sugar (glucose) has been formed, as in the similar experiment with cane-sugar, This experiment is the basis of the commercial manufacture of glucose.

\section{Dextrin, $\left(\mathrm{C}_{6} \mathrm{H}_{10} \mathrm{O}_{5}\right)_{\mathrm{n}}$.}

As explained above, a number of compounds are included under this head. They are the first hydration products of starch. The commercial dextrin is prepared by heating starch to $150-160^{\circ}$ with or without water; also by drying starch at $100^{\circ}$ previously suspended in very dilute nitric acid; or by treatment with acids or malt and subsequent precipitation with alcohol. The behavior of the several varieties of dextrin to iodine has been indicated above and will be demonstrated in connection with the work on saliva.

Unlike starch, dextrin is very readily soluble in water. The solution is not fermented by yeast, but must first be changed by hydration to maltose. Test a one per cent. solution as follows:

1.--To some of the solution add tannic acid-no precipitate: dis tinction from starch. gelatin, albumin.

2.-To some of the solution add a drop or two of iodine solution. What is the result? And to what is it due?

3.- To some of the solution add Fehling's solution and boil. Ordinary dextrin contains more or less of reducing substances. If pure, no reaction would take place.

\section{Glycogen, $\left(\mathrm{C}_{6} \mathrm{H}_{10} \mathrm{O}_{5}\right)_{\mathrm{n}}$.}

This carbohydrate was first discovered in the liver and has since been shown to be present, in greater or less amount, in all the tissues of the animal kcay. The anoun 
of glycogen in the liver will vary according to the food. Ordinarily it constitutes from $1-4$ per cent. but may, after a rich carbohydrate diet, amount to 12-16 per cent. In fresh muscle it amounts to about 0.6 per cent., and disappears from the muscle as a result of work or starvation. It is present in Liebig's meat extract ( $1-1.5$ per cent.). Although present in small amounts in normal blood, it is considerably increased after extirpation of the pancreas. It is present in larger amount in pus, and in leucocytes. Undoubtedly it is a constituent of all living animal cells. It is abundant in embryonic tissue, and the liver of a new-born dog has been found to contain as much as 11 per cent. It is present in considerable quantity in molluscs, notably in oysters. It has been found in certain plants; fungi, as truffles; also in mucor and in the yeast.

Glycogen is related to dextrin and to amylodextrin or soluble starch. It has the same percentage composition as starch or dextrin. The exact formula is not known. It would seem that the multiple $n$ in the above formula is 6 , although some place it at 10 . Glycogen is derived from the food, more especially the carbohydrates. The excess of carbohydrates, whether starch or various sugars, is promptly stored up in the liver to be given off under the influence of ferments according to the need of the body. Exclusively proteid diet likewise gives rise to glycogen.

Various diastatic ferments, such as are present in malt, saliva, pancreas, blood, liver, etc., invert glycogen. The change is similar to that which starch or soluble starch undergoes under like conditions. That is to say, erythrodextrins, achroodextrin, isomaltose, maltose, and eventally glucose form. On heating with water at high temperature, or with dilute acids, a similar hydration results. Owing to the action of these ferments of the body, it follows that in dead liver or muscle the amount of glycogen rapidly decreases, and is replaced by a dextrin body, or by maltose or glucose. 
The following table shows the relation of glycogen to sugar in the liver of a rabbit at different periods after death (Girard):

\begin{tabular}{|c|c|c|c|}
\hline & $10 \mathrm{~min}$. & 24 hrs. & 48 hrs. \\
\hline $\begin{array}{l}\text { Sugar, per cent.......... } \\
\text { Glycogen, per cent....... }\end{array}$ & $\begin{array}{l}0.75 \\
9.56\end{array}$ & $\begin{array}{l}3.58 \\
6.35\end{array}$ & $\begin{array}{l}3.85 \\
4.28\end{array}$ \\
\hline
\end{tabular}

The action of the diastatic ferments is most marked in neutral or very slightly acid solutions. A one per cent. solution of sodium carbonate inhibits the change and so does the acidity of a solution of $\mathrm{CO}_{2}$. It is possible that the carbonic acid prevents or retards the hydration of glycogen in the body. Glycogen is not affected by yeast.

Glycogen is an amorphous, white, tasteless powder which dissolves in warm water to form an opalescent liquid. The opalescence disappears on the addition of an acid or alkali. On the addition of iodine the solution becomes red or brown (erythrodextrin). The color, like that of starch iodide, disappears on heating. The solutions are strongly dextro-rotatory. It is precipitated from impure solution by alcohol.

1.--Isolation of Glycogen.-The following method gives the best results. It may be applied to $50 \mathrm{~g}$. of fresh liver, or to $1 / 2$ pint of oysters. The material is cut up as fine as possible. If liver is used it can be put through a sausage machine. To the material then add 10 parts of boiling water slightly acidulated with acetic acid. Strain the opalescent liquid through muslin. This liquid contains, besides glycogen, some proteids and gelatin. To remove the latter, first concentrate to a small volume, then add alternately a few drops of $\mathrm{HCl}$ and of potassium mercuric iodide till a precipitate ceases to form. Finally filter off a little of the liquid and test it with acid and reagent to make sure that all the proteids are precipitated. If this is the case, strain the liquid through muslin, then filter through paper, and to the filtrate add two volumes of alcohol and stir thoroughly. Allow the glycogen to settle, then filter off, wash with dilute alcohol (2 parts alcohol to 1 part water). Finally transfer to a beaker, cover with absolute alcohol and let stand an hour or more. Then filter off the 
glycogen, fold the filter and gently squeeze off excess of alcohol, finally press between several layers of filter paper till dry. Powder, if necessary.

The reagent employed above is prepared. by adding mercuric iodide to a warmed 5 per cent. solution of KI till it ceases to dissolve. The liquid is then cooled and filtered.

With glycogen isolated as above make the following tests:

1.- To some glycogen in a small beaker add 20-30 c.c. of water and warm. The glycogen dissolves, forming an opalescent liquid. Resemblance to soluble starch.

2. - To a portion of the solution just obtained add a few drops of iodine solution (in potassium iodide). A reddish-brown color forms. Then heat the contents of the tube. The color disappears, to reappear on cooling. Resemblance to erythrodextrin and to starch-iodide. The presence of pepton interferes.

3.-Boil another portion of the glycogen solution with Fehling's solution. Note the result.

4.-To some of the glycogen solution add a few drops of $\mathrm{HCl}$ and boil a few minutes. Glycogen-dextrin, dextrose. Then cool and neutralize, and test a portion with iodine; another portion with Fehling's solution. Conpare with Exp. 2 and 3.

5.-To some of the glycogen solution add about 1 c.c. of saliva and mix. At the end of 10 minutes examine a portion with iodine: another portion with Fehling's solution. What is the result?

\section{Cellulose, $\left(\mathrm{C}_{6} \mathrm{H}_{10} \mathrm{O}_{5}\right)_{\mathrm{n}}$.}

Cellulose, or wood-fiber, is present in all higher plants and as a rule in the lower plants, including fungi and bacteria. It largely makes up the walls of the cell. Cellulose is probably formed by the protoplasm of the cell out of the carbohydrates that result from the assimilation of the carbonic acid of the air. The molecule of cellulose is probably much more complex than that of starch. Moreover, it is probable that there are various distinct cellulose bodies. Tunicin or animal cellulose is found in some lower 
animals, as the tunicata, and is identical with plant celluose and yields on decomposition dextrose. Cellulose has 'been reported in the lungs, blood and pus of tuberculous patients (Freund).

Cellulose is characterized by its difficult solubility. It is insoluble in water, alcohol, dilute acids or alkalis. It is soluble in an ammoniacal solution of copper oxide or Scliweizer's reagent, and from this solution it can be precipitated, unaltered, in an amorphous form by acids, alco. hol or water. Cellulose is furthermore characterized by its reaction with iodine and concentrated sulphuric acid. Treated with concentrated sulphuric acid and with iodine it gives a blue color. This is due to a so-called amyloid substance which, however, is not identical with the amyloid found in the animal body. Indeed the latter is not a carbohydrate but probably a proteid. In place of $\mathrm{H}_{2} \mathrm{SO}_{4}$, zinc chloride can be used.

It does not melt on heating but turns brown and event. ually decomposes, yielding various products, some of which have considerable commercial importance. Thus, there is formed methyl alcohol (wood-spirit), acetic acid (woodvinegar), and creosote (wood-tar).

Concentrated sulphuric acid dissolves cellulose, and if this solution is treated at once with water a gelatinous precipitate of soluble cellulose or amyloid forms. If the acid is allowed to act longer, or the solution is heated, no precipitation takes place on dilution. When paper is rapidly immersed in concentrated sulplunic acid to which $1 / 4$ its volume of water has been added, and is then washed in water, amyloid which is first formed is precipitated on the paper. The result is the tough parchment paper.

When the solution of cellulose in sulphuric acid is allowed to stand for some time, then diluted with water and boiled, glucose forms. Some kinds of cellulose yield mannose. Unlike starch, boiling with dilute $\mathrm{H}_{2} \mathrm{SO}_{4}$ lias but little effect. 
With concentrated nitric acid, or a mixture of nitric and sulphuric $(1-3)$ acids, it forms various so-called nitrocelluloses. These compounds are made use of in several important preparations. Thus, collodium, which is used in surgery and in photography, is a mixture of tri- and tetranitro-cellulose dissolved in ether. Gun-cotton or pyroxylin is a mixture of the tetra- and hexa-nitrates. Smokeless powder, which has revolutionized modern warfare, may be pure gun-cotton, or gun-cotton mixed with nitrate of barium and potassium, or gun-cotton mixed with nitro-glycerin in different proportions (nobelite, cordite, explosive gelatin). Powders are also made out of nitro-phenol (picric acid) and out of nitro-naphthalens.

A mixture of nitro-cellulose and cellulose can be drawn out into long glistening threads resembling silk (woodsilk). The cellulose which is the basis of ordinary paper is obtained from wood by heating it with calcium sulphite under pressure.

Cellulose has been obtained in the shape of sphaero crystals or minute needles. Cotton and linen threads and Swedish filter paper are practically pure cellulose. In the dry condition it is permanent but in the presence of water it readily undergoes, under the influence of bacteria, fer mentative decomposition giving rise to marsh gas. This bacterial decomposition takes place in the intestines and marsh gas, acetic and butyric acids are formed. The cellu. lose of the food increases the peristaltic action of the intestines and consequently considerable nitrogen may escape absorption.

1.-Examine under the microscope and sketch, cotton, linen, silk and wool fibers, also hair. The linen fibers are a hollow tube with a thick wall and hence retain their shape, whereas the cotton fibers have thin walls which readily collapse and produce the twisted character.

2.-Tear up a little "washed" filter paper into small shreds (or use cotton) and warm with fresh Schweizer's reagent. The cellulose 
dissolves. Acidulate the solution with acetic acid when it precipitates in an amorphous form. Schweizer's reagent is obtained by adding sodium hydrate to a solution of copper sulphate in the presence of $\mathrm{NH}_{4} \mathrm{Cl}$. The copper hydrate precipitate is filtered off, washed and dissolved in $20 \%$ ammonium hydrate.

3.--Immerse some shreds of "washed" filter paper, or cotton, in a strong solution of potassium hydrate (1-1). Allow the reagent to act for 10-15 minutes till the paper becomes gummy. Then transfer to a dish of water, and wash thoroughly, then acidulate with a little dilute hydrochloric acid and add some iodine solution. A blue color, due to amyloid, results.

4. - To some cotton or shreds of paper add 5-10 c.c. of cold sulphuric acid. As soon as solution results take a portion of it, cool and dilute with water. A gummy precipitate of amyloid forms. Add iodine solution, it colors blue.

Allow the remainder of the acid solution to then stand for some time, then dilute with water and boil for $1 / 2$ hour; cool, neutralize with potassium hydrate and test with Fehling's solution for sugar.

5.-Dilute some sulphuric acid with one-half its volume of water, and cool the mixture. Then immerse, for a few seconds, an ordinary filter paper: remove at once and wash in tap-water. The tough parchment-paper results.

\section{The Mitscherlich Polarimeter.}

The instrument consists of two Nicol prisms, the polarizer and analyzer, enclosed in brass tubes and supported in such a way that they can be rotated; the tube containing the analyzer has a pointer attached which measures the amount of its rotation upon a circle graduated in degrees. Between the two, Nicols is placed the observation tube, a brass tube exactly $200 \mathrm{~m} . \mathrm{m}$. long, the ends of which are closed with glass plates; this holds the solution to be tested.

\section{Adjust the instrument as follows:}

Place a lamp behind the polarizer and fill the observation tube with distilled water; set the pointer at $0^{\circ}$, and then rotate the polarizer until the field becomes darkest. The polarizer must not be moved again.

As the instrument now stands, the two Nicols have their section at right angles. If the analyzer is rotated, one way or the other, the field gradually becomes brighter and is brightest when the pointer is 
at $90^{\circ}$, the sections of the prisms now being parallel. The field will be dark again at $180^{\circ}$.

Starting with the instrument adjusted as above, fill the observation tube with the solution to be tested. If this has the power of rotating the plans of polarization, the field appears bright. The analyzer must now be turned to the right or left till the field again becomes darkest, thus compensating for the rotatory power of the solution. This shows whether the substauce is dextro or lavo-rotatory. By knowing the length of the tube, the concentration of the solution, and the number of degrees through which the analyzer was turned, the Specific Rotatory Power can be calculated.

\section{The Soleil-Ventzke Saccharimeter.}

This instrument is used only for the purpose of determining the percentage of cane sugar in a given sample.

It consists of two Nicol prisms, the analyzer and polarizer, and the observation tube placed between them. Between the polarizer and source of light is the regulator, a Nicol prism and a quartz plate, for the purpose of changing the colors. Between the analyzer and tube is the compensator: this consists of two wedge-shaped plates, one fixed, and the other capable of being slid over it, thus increasing or diminishing the thickness of the crystal through which the polarized ray passes. Fastened to the movable plate is a scale graduated so that it can be read to tenths of one per cent; the reading is done by means of a vernier and telescope. The source of light is a lamp placed back of the polarizer.

With the scale reading at $0^{\circ}$, and the tube filled with distilled water, the field appears as a colored circle divided vertically, and both halves of exactly the same shade of color. This color may be changed by simply rotating the regulator. For most persons the "sensitive tint" is a rose violet.

Now fill the tube with a solution of cane sugar, prepared as given below. The plane of polarization is deviated, and the two disks are of different colors. Then turn the screw of the compensator till the disks are again of the same shade, thus compensating for the deviating effect of the sugar. The percentage of cane sugar can now be read distinctly from the scale.

The instrument is so made that with a solution of pure cane sugar containing 26,048 grms. in 100 c.c. at $17.5^{\circ}$ c. the reading will be $100 \%$. Consequently, in making a determination, dissolve 26,048 grms. of the substance in distilled water at $17.5^{\circ}$, and dilute to 100 c.c. The reading obtained will be the percentage of cane sugar in the substance. 


\section{CHAPTER III}

\section{PROTEINS.}

Representatives of this group are found in every living organism, animal or vegetable. They are present within the cell as an essential, integral part of protoplasm, and are likewise always present in the fluids without the cell. In addition to $\mathrm{C}, \mathrm{H}$, and $\mathrm{O}$ they contain $\mathrm{N}$ and $\mathrm{S}$, and some have $\mathrm{P}$ and even $\mathrm{Fe}$. In the plant these substances are made from the inorganic compounds, ammonia, nitrates, nitrites, sulphates, etc., whereas in herbivorous animals they are derived from the vegetable food in which, during life, these bodies have been elaborated. The carnivorous, or omnivorous animal in turn builds up these products from those contained in the meat, or mixed diet respectively. The animal organism cannot make protoplasm, hence live and grow, on inorganic nitrogen, sulphur and phosphorus. These elements are supplied only through the proteins existing ready made in our food. However, not all the members of this group are capable of sustaining life; this is notably true of the albuminoids. Those members are of utility as real food which, when acted upon by the digestive fluids, yield peptons which in turn can be reconstructed into serum albumin and globulin.

The members of this group constitute by far the most complex bodies known to the chemist. While their percentage composition can often be determined quite readily it is otherwise with their molecular composition. Undoubtedly thousands of atoms may be contained in one molecule. On complete cleavage with acids they yield final products as ammonia; organic bases as lysin, histidin, arginin; and amido acids as leucin, tyrosin, etc.

It may be said in this connection that considerable con. 
fusion exists regarding the usage of certain terms. In English works the term proteid is used; first, as a general designation for the entire group, i. e., in place of protein; second, to denote one of the subgroups, namely, the albuminous bodies. On the other hand German writers use it to designate the more complex albuminous bodies.

The following table is essentially that of Wroblewski.

\section{CLASSIFICATION OF PROTEINS.}

I. Albuminous Bodies; these contain $\mathrm{C}, \mathrm{H}, \mathrm{N}, \mathrm{O}, \mathrm{S}$; some have $\mathrm{P}$.

1.-Albumins = serum albumin, egg albumin,, lactalbumin, muscle albumin, plant albumin, etc.

2.-Globulins = serum globulin, egg globulin, lactoglobulin, fibrinogen, myosin, plant globulins, vitellin (?) etc.

3. - Soluble in alcohol $=$ chiefly found in plants.

4.-Albuminate.

5. - Acid albumin $=$ syntonin, etc.

6.-Coagulated albuminous bodies = fibrin, paracasein, heat coagulated albumins, etc.

II. Proteids, or complex albuminous bodies, which on cleavage yield members of the preceding group.

1. - Glycoproteids = mucin, mucoids.

2.-Hæmoglobins.

3.-Nucleo-albumins.

4.-Caseins.

5.-Nucleins.

6.-Amyloid.

7.-Histon (?).

III. Albuminoids, or albumin-like bodies.

a. Skeletal or support substances.

1.--Keratins.

2.-Elastins.

3. - Collagens $=$ collagen, gelatin, etc.

b. Albumoses and peptons.

c. Enzymes.

1.-Proteolytic = pepsin, trypsin, papayotin, etc.

2.-Amylolytic = diastase, invertin, etc.

3.-Fat-splitting = steapsin.

4.-Glucoside-splitting.

5.-Amid-splitting = urase, etc.

6.-Coagulating $=$ rennet. 


\section{Egg Albumin.}

Apply the following tests which are, more or less, general reactions for proteins to a $2 \%$ solution, unless otherwise indicated, of egg albumin. A white of an egg is carefully poured into an evaporating dish, then cut up with scissors, and 20 c.c. of the liquid is diluted to 1 liter $(1-50=2 \%)$. After thorough shaking in a cylinder the liquid is filtered and the clear filtrate employed for the tests. Observe the frothing of the liquid on shaking.

Dilute another portion of 2 c.c. of the egg albumin to 10 c.c. and shake thoroughly $(1-5,=20 \%)$; also, dilute 2 c.c. to 20 c.c. and shake till thoroughly mixed $(1-10,=10 \%)$.

\section{COLOR REACTIONS OF PROTEIDS.}

The following color tests $(1-6)$ are general reactions for proteids:

1.-Biuret test.-To the albumin solution $(1-50)$ add an equal volume of strong sodium or potassium hydrate. Then heat to boiling and add 1-2 drops of very dilute $\mathrm{CuSO}_{4}$ solution. The solution becomes colored, pink to violet, according to the amount of copper sulphate used. An excess of copper must be avoided. Salts of nickel give a similar reaction.

Repeat the test omitting the heat. What is the result?

All proteids give the biuret test, some more readily than others. The hydrated proteids, albumoses and peptons, give the test in the cold. Gelatin gives in the cold, a bluish violet color, not purple red as in the case of peptons.

The biuret reaction would indicate that proteids contain the biuret or urea group. Diamids, such as oxamid and its derivatives, however, give similar biuret reactions, and it is possible that such diamid groups are present in the proteid molecule. It is possible to remove the diamid group and the modified proteid that results no longer gives the biuret reaction (Schiff).

2. - Millon's reaction. - To some of the albumin solution $(1-50)$ add a few drops of Millon's reagent. A white precipitate forms which on 
boiling for $2-3$ minutes becomes colored red. The liquid may become likewise red.

This reaction is due to the aromatic nucleus contained in the proteid molecule. It is given by phenol, tyrosin, etc.

Millon's reagent is prepared by dissolving in the cold one part of mercury in one part by weight of concentrated $\mathrm{HNO}_{3}$ (1.40). Gentle heat is finally applied and when all is dissolved two volumes of water are added. The mixture is allowed to stand for some hours and the clear liquid is then decanted from any crystalline sediment that may be deposited.

3.-Xanthoproteic reaction. - To some of the albumin solution $(1-50)$ add an equal volume of concentrated $\mathrm{HNO}_{3}$. Then heat to boiling till the precipitate turns yellow or gives a yellow solution. Cool, and add an excess of $\mathrm{NH}_{4} \mathrm{OH}$ or $\mathrm{NaOH}$. The color changes to an orange yellow.

This test can be always incidentally applied to the precipitate or liquid obtained in Heller's test, or in the nitric acid and heat test (I, 9 and $9 a)$.

4.-Adamkiewicz's reaction.-To 2 c.c. of concentrated $\mathrm{H}_{2} \mathrm{SO}_{4}$ add about 4 c.c. (two volumes) of glacial acetic acid and mix. To the mixture add one drop of the undiluted egg albumin. The liquid changes, slowly on standing, more rapidly when slightly warmed to a beautiful reddish violet color. The reaction is not given by gelatin or gelatin pepton.

The presence of water interferes with the reaction. It is therefore desirable to use the dry proteid or one drop of a concentrated solution. The spectrum of the solution resembles that of urobilin.

5.-Liebermann's reaction. - To about 3 c.c. of concentrated $\mathrm{HCl}$ add 1-2 drops of undiluted egg albumin. Boil the liquid for several minutes. A pink to a violet color develops. Too much water interferes with the reaction.

6.-Heat some albumin with concentrated $\mathrm{H}_{2} \mathrm{SO}_{4}$ and a little sugar. A red color results. Excess of sugar interferes by imparting a dark caramel color to the liquid.

The proteid molecule contains one or more aromatic groups. This is seen in the fact that on decomposition 
three distinct groups of aromatic bodies form. Thus we may have 1st, the oxy-phenyl group, represented in phenol and in tyrosin; 2nd, the phenyl group, represented in phenylacetic acid; and $3 \mathrm{rd}$, the indol group represented by indol and skatol. The xanthoproteic reaction is due to the formation of nitro-products out of the members of the 1st group. Millon's reaction is also due to the presence of the 1st group of compounds. It is not given by the $2 \mathrm{nd}$ or 3rd groups. The Adamkiewicz reaction is due to the 3rd group of products. On the other hand the Liebermann's reaction is apparently not due to the aromatic group.

\section{PRECIPITATION REACTIONS OF PROTEIDS.}

7.-Take four test-tubes, label and equip as follows: To tube 1 add 1-2 c.c. of the undiluted egg albumin; to tube 2 add 5 c.c. of the egg albumin solution, $1-5$; to tube 3 add 5 c.c. of the egg albumin solution, 1-10: and, to tube 4 add 5 c.c. of the solution, $1-50$. Immerse the four tubes in a boiling water-bath for 5-10 minutes, after which examine and note the results. Test the reaction of tubes $2,3,4$. Tube 1 coagulates solid, whereas tubes $2,3,4$, are more or less opalescent but far from coagulation. Dilution of egg albumin with water renders it non-coagulable by heat. Compare with similar test with blood-serum.

8.-In each of four test-tubes place 5 c.c. of the egg albumin solution $(1-50)$. To tubes 1 and 2 add respectively 1 c.c. and 0.2 c.c. of a $10 \%$ $\mathrm{NaCl}$ solution. To the tubes 3 and 4 add respectively one and five drops of a $1 \%$ ace tic acid solution (1 c.c. of a glacial ace tic diluted to 100 c.c.) To a fifth tube containing 5 c.c. of egg albumin solution $(1-10)$ add 1 c.c. of a $10 \% \mathrm{NaCl}$ solution. Immerse the five tubes in a boiling water-bath for about five minutes, then examine and note the results. Test the reactions of tubes 3 and 4 . In experiment 7 , above, tube 4 , which can be considered as a control for this experiment, on exposure to $100^{\circ}$ shows only a very slightopalescence. The addition of a small amount of $\mathrm{NaCl}$ increases the opalescence (tube 2 ); the same amount of $\mathrm{NaCl}$ as in tube 1 , added to a stronger solution of albumin (tube 5 ) brings on coagulation on heating; and a larger amount brings on partial coagulation on the walls of the tube (tube 1). Now add one or two drops of the $1 \%$ ace tic acid to tubes $1,2,5$, and to tube 4 add 1 c.c. of $10 \% \mathrm{NaCl}$ and heat argain. Prompt and complete coagulation results. The liquid is 
clear. In tube 3 the addition of one drop of the diluted acid, thus changing the liquid to a very slight acid reaction, suffices to produce on heating a precipitate. A very slight excess of the acid (as in tube 4) prevents coagulation by heat. If $\mathrm{NaCl}$, however, is added coagulation promptly results.

In attempting to remove albumin completely from a solution, as in the case of urine, it should be remembered that very dilute solutions must be barely acidulated with acetic acid. Furthermore, that the presence of NaCl favors coagulation on subsequent heating.

Albumin coagulates in a slightly acid or neutral solution, especially in the presence of a neutral salt as $\mathrm{NaCl}$. Globulin requires a neutral salt to keep it in solution and this moreover favors coagulation on heating. Hæmoglobin on heating decomposes into hæmatin and globulin; the latter as just stated coagulates on heating in the presence of a neutral salt. Nucleo-albumin is coagulated or thrown out of solution by acetic acid alone. The albumoses, as will be seen later, are precipitated by $\mathrm{NaCl}$ and the precipitate unlike albumin and globulin dissolves on heating. Peptons are not coagulated by heat.

9. - To about 5 c.c. of the albumin solution $(1-50)$ add an equal volume of concentrated $\mathrm{HNO}_{3}$ so that the two liquids do not mix. This is done by allowing the acid to slowly run down the side of the inclined tube. A white cloud forms at the zone of contact of the two layers (Heller's test). Now mix the two liquids and gently warm. A flocculent precipitate separates. Now heat the mixture to boiling. In a short time the precipitate dissolves, acid albumin forms, and the liquid is colored yellow. Cool the liquid and add an excess of $\mathrm{NH}_{4} \mathrm{OH}$. An orange yellow color results (Xanthoproteic reaction).

$\mathrm{Egg}$ albumin is therefore coagulated by $\mathrm{HNO}_{3}$. The solution of this precipitate on boiling shows a distinction between this and the serum proteids.

Mineral acids, such as $\mathrm{HNO}_{3}$, coagulate albumin and globulin. The albumoses are precipitated by $\mathrm{HNO}_{3}$ especially if $\mathrm{NaCl}$ is present, but the precipitate readily dissolves on the application of heat and reappears on cooling. Peptons are not precipitated by acids. 
9a.-The test employed most often for the detection of albumin (and globulin) in the filtered urine is the corrgulation, or nitric acid and hert test. The reaction when properly carried out is exceedingly delicate. The best procedure is as follows: To the urine add some concentrated $\mathrm{HNO}_{3}$ so as to form two layers (see above). A precipitate or cloud indicates albumin. Now mix the two liquids and heat. A persistent flocculent precipitate is due to albumin, or globulin, or both. Should it be necessary to decide whether this precipitate is due in part or whole to albumin, it can be done by saturating the urine with $\mathrm{MgSO}_{4}$ according to directions given under Globulin Test 5.

If heat is applied direct to the urine a precipitate of phosphates may form. This, however, dissolves readily in $\mathrm{HNO}_{3}$. If the urine is alkaline the $\mathrm{HNO}_{3}$ should be added first to prevent formation of alkali-albuminate.

Apply the test as just given to some albuminous urine.

10. - To about 5 c.c. of the albumin solution $(1-50)$ add $1-2$ drops of strong acetic acid, then add 1-2 drops of potassium ferrocyanide. A roluminous precipitate forms.

This is a very delicate test for proteins. It is not given, however, by peptons. The presence of $\mathrm{NaCl}$ favors the precipitation of the albumoses. Moreover the albumose precipitate dissolves on heating and reappears on cooling.

This test and the nitric acid heat test, given above, are commonly employed for the detection of albumin in the urine.

If, in the case of urine, the amount of the precipitate is small and its nature doubtful it should be transferred to a filter and washed. The precipitate can be transferred by means of a glass rod to a test-tube and Millon's reagent added. If on heating a reddish coloration forms it indicates the presence of a proteid. Another procedure is to add $1 / 2$ c.c. of the boiling Millon's reagent direct to the precipitate on the filter (Winternitz).

11.-Strongly acidulate some of the albumin solution $(1-50)$ with HC'l, then add a few drops of phosphotungstic acid. A heavy white precipitate results. It is given by all proteids. Phosphomolybdic acid behaves in a similar manner. 
12.- Acidulate another portion as above and add a few drops of a solution of potassium mercuric iodide. Note the results. Why was this reagent used in the preparation of glycogen?

13. - To a portion of the albumin solution (1-50) add 1-2 drops of tannic acid. What is the behavior of tannic acid to starch? To dextrin?

14. - To another portion of the solution add a few drops of picric acid. A yellow voluminous precipitate forms. This reagent is used in Esbach's method for the estimation of albumin in urine.

The reagents employed in tests $10-14$ inclusive are sometimes spoken of as alkaloidal regents because of their reactions with the vegetable alkaloids and other bases. They are general reagents for proteids.

15. - To 3 c.c. of the albumin solution $(1-50)$ add one drop of mercuric chloride. A heavy white cloud or precipitate results. Divide the cloudy liquid into two portions.

(a). To one add an equal volume of a $10 \%$ solution of $\mathrm{NaCl}$. The precipitate promptly dissolves even if mercury is in large excess.

(b). To the other portion add two volumes of the undiluted egg albumin solution and mix. The precipitate dissolves if too much mercury has not been added.

16. - To another small portion of the egg albumin solution add 1-2 drops of dilute lead acetate and note the result.

17. - To a portion of the solution add 1-2 drops of silver nitrate. A voluminous white precipitate forms which on the addition of $\mathrm{NH}_{4} \mathrm{OH}$ dissolves.

Experiments 15, 16, 17, are made with the salts of the heavy metals which precipitate most of the proteids. Why is the white of eggs administered in case of poisoning with corrosive sublimate or with salts of other heavy metals? Why should a stomach pump be subsequently used?

18. - To about 3 c.c. of the albumin solution $(1-50)$ add $10-15$ c.c. of strong alcohol and mix. If no precipitate forms, but merely a cloudiness, then add $1 / 4-1 / 2$ c.c. of a $10 \%$ solution of $\mathrm{NaCl}$. A voluminous white precipitate results. 
Alcohol added in large excess (10 volumes or more) precipitates all proteids. The presence of $\mathrm{NaCl}$ favors the precipitation.

19. - Place 10 c.c. of egg albumin solution $(1-50)$ in a small beaker or test-tube on foot. Add about $7 \mathrm{~g}$. of powdered $\left(\mathrm{NH}_{4}\right)_{2} \mathrm{SO}_{4}$ and immerse in a water-bath at about $35^{\circ}$ for half an hour. Stir frequently till the salt ceases to dissolve. Notice the heavy white precipitate that forms (albumin and globulin). When saturated transfer the contents to a dry filter. Test the filtrate:

(a). By acetic acid and heat.

(b). By the biuret test, in the cold.

Are the results positive or negative? Explain.

20 .-Place 10 c.c. of the egg albumin solution $(1-50)$ in a small beaker, as above, add about $12 \mathrm{~g}$. of $\mathrm{MgSO}_{4}$ and digest, with frequent stirring, at $35^{\circ}$ for about half an hour. Observe that only a very slight cloud or precipitate forms (globulin). Filter through a dry filter and to the filtrate apply tests $\alpha$ and $b$ as in Experiment 19. What proteid is present in the filtrate? In the biuret test a large excess of $\mathrm{NaOH}$ should be added owing to the precipitate of $\mathrm{Mg}(\mathrm{OH})_{2}$ that forms.

On saturation with $\mathrm{MgSO}_{4}$ globulin is precipitated whereas albumin remains in solution. Saturation with $\left(\mathrm{NH}_{4}\right)_{2} \mathrm{SO}_{4}$ throws down albumin, globulin, and albumose, but not pepton.

21.-Determination of the coagulation point of albumin.-Place about 5 c.c. of the undiluted egg albumin in a test-tube. Close the tube with a stopper through which passes a thermometer. The bulb of the thermometer should nearly touch the bottom of the tube and should be completely immersed in the albumin. Suspend the tube thus equipped in a large beaker of water. Fully two-thirds of the tube should be immersed. Heat gradually the water in the beaker and stir continually by means of a glass rod bent at right angles. Note the temperature at which the albumin clouds. The albumin then becomes sticky; does not flow readily when inclined and finally becomes solid. Note the coagulating point of egg albumin. 
22. - To 20 c.c. of the $2 \%$ albumin solution add $2-3$ drops of concentrated $\mathrm{HCl}$ and boil. No precipitate forms owing to the formation of an acid albumin. Cool the solution.

(a). To a portion add an excess of concentrated $\mathrm{HCl}$, a precipitate forms that is difficulty soluble in excess.

(b). In the remainder of the solution place a litmus paper and add, drop by drop, very dilute NaOH. Mix the contents well after each addition of alkali. As soon as a precipitate or cloud forms note the reaction of the liquid. The precipitate of albuminate forms while the liquid is still acid. After the precipitate has formed add 2-3 drops more of the dilute $\mathrm{NaOH}$. It dissolves at once to form an alkali albuminate.

23. - To 10 c.c. of the albumin solution add 1-2 drops of $\mathrm{NaOH}$ solution and warm gently for a few minutes. An alkali albuminate forms. Raise the solution to boiling. It does not coagulate. Cool, add litmus paper and carefully neutralize. as above, with dilute $\mathrm{HCl}$. What is the result? What is the effect of a slight excess of $\mathrm{HCl}$ ?

Report the results obtained with egg albumin and with the proteids subsequently to be studied in a tabular form such as the following:

\begin{tabular}{l|l|l|l|l|l|l}
\hline \hline & \\
\end{tabular}




\section{Serum Albumin and Serum Globulin.}

Globulin.-It is usually associated with albumin, though it may sometimes, as in the urine, occur alone. The tests given for albumin, as well as the general proteid reactions, are also given by globulin. For the separate recognition of albumin and globulin, when both are present in solution, it is necessary to resort to precipitation by either of the following methods:

1.-Piecipitation with $\mathrm{MgSO}_{4}$.- To 10 c.c. of blood-serum, in a small beaker or test-tube on foot, add 10 c.c. of saturated $\mathrm{MgSO}_{4}$ and $15 \mathrm{~g}$. of powdered $\mathrm{MgSO}_{4}$. Immerse the beaker or tube in a water-bath at a temperature of $30-35^{\circ}$. Stir frequently for $1 / 2-1$ hour, until the $\mathrm{MgSO}_{4}$ ceases to dissolve and the liquid is saturated. The globulin is thrown out of solution. Transfer the liquid and precipitate to a small filter. Save the filtrate ( $r)$ which contains albumin. When the liquid has drained through wash the residue $2-3$ times with saturated $\mathrm{MgSO}_{4}$. Finally spread out the filter on a flat surface, transfer the precipitate by means of a spatula to about 20 c.c. of water. Globulin when pure does not dissolve in water, but in this case, owing to the presence of salts, it dissolves.

Filter the solution and the clear filtrate $(b)$ containing the globulin is reserved for experiment 3.

Apply to the original filtrate $(a)$ which contains serum albumin the tests enumerated in the table. Note the results. Wherein does egg albumin differ from serum albumin? Boil a portion of the serum albumin solution to coagulate the albumin. Filter and apply the biuret test to the filtrate. What is the result?

2.-Precipitation by semi-saturation with $\left(\mathrm{NH}_{4}\right)_{2} \mathrm{SO}_{4}$ - - To 10 c.c. of blood serum as above, add 10 c.c. of saturated $\left(\mathrm{NH}_{4}\right)_{2} \mathrm{SO}_{4}$. Immerse in a water-bath at $30-35^{\circ}$ for about $1 / 2$ hour and stir frequently. Then transfer the contents to a small filter. Save the filtrate (A) which contains albumin. Wash the residue on the filter $2-3$ times with semi-saturated $\left(\mathrm{NH}_{4}\right)_{2} \mathrm{SO}_{4}$. Finally spread out the filter on a flat surface, transfer the precipitate to about $20 \mathrm{c} . \mathrm{c}$. of water. The globulin precipitate dissolves for the reasons given above under 1.

Filter the solution and combine the clear filtrate (B) with the corresponding filtrate from experiment 1 . The resulting solution is used for experiment 3. 
The precipitate obtained by this method is larger than that obtained by the $\mathrm{IgSO}_{4}$ method. The liquid filters much easier.

3.- Separation of salts from globulin by dialysis.-Place the combined filtrates ( $b$ and $B$ ) in a dialyzer and dialyze against running water. Every day remove a few drops of the liquid from the dialyzer with a pipette and add this to some dilute $\mathrm{BaCl}_{2}$ solution. The dialysis should continue till the sulphates are completely removed. This may require $3-5$ days. In warm weather to prevent decomposition it is well to add a few drops of thymol.

When the sulphates have dialyzed out the globulin is thrown out of solution as a white granular precipitate. Now transfer the contents of the dialyzer to a small beaker. Pour into the dialyzer about 20 c.c. of a $2 \%$ solution of $\mathrm{NaCl}$ and gently agitate to dissolve any precipitated globulin. Add this saline solution to the contents of the beaker and stir till the globulin dissolves. Finally allow the liquid to stand for a while, then filter. The clear filtrate now contains pure globulin. Observe the frothing of the liquid on shaking.

To this solution of globulin apply the tests enumerated in the table on page 46. Make careful records of the results obtained. The presence of $\mathrm{NaCl}$ will interfere with tests 16 and 17.

Boil a portion of the globulin solution to coagulate the globulin. To the filtrate apply the biuret test.

4.-To 10 c.c. of blood-serum add an equal volume of saturated $\left(\mathrm{NH}_{4}\right)_{2} \mathrm{SO}_{4}$. Then add $8 \mathrm{~g}$. of powdered $\left(\mathrm{NH}_{4}\right)_{2} \mathrm{SO}_{4}$ and immerse in a water-bath at $30-35^{\circ}$, stirring frequently, for about $1 / 2$ hour. The liquid becomes saturated with $\left(\mathrm{NH}_{4}\right)_{2} \mathrm{SO}_{4}$ and a precipitate forms. Finally transfer to a filter. Notice the perfect clearness of the filtrate.

Test a portion of the filtrate by boiling; another portion with tannic acid.

To another portion apply the biuret test in the cold.

The absence of proteids in the filtrate demonstrates that albumin and globulin are completely precipitated on saturation with $\left(\mathrm{NH}_{4}\right)_{2} \mathrm{SO}_{4}$. The absence of a biuret reaction indicates the absence of a pepton.

$5^{*}$.-Detection of globulin in the urine.-As indicated under albumin (Experiment $9 a$, page 43), the ordinary tests for albumin are also given by globulin. In order to ascertain positively which of the two,

* Experiments or methods designated by an asterisk are omitted in the ordinary laboratory course. 
or if both are present, it is necessary to resort to the saturation method with $\mathrm{MgSO}_{4}$. For this purpose 100 c.c. of the urine can be taken and neutralized. $120 \mathrm{~g}$. of powdered $\mathrm{MgSO}_{4}$ are then added and the liquid kept at $30-35^{\circ}$, with frequent stirring, till the $\mathrm{MgSO}_{4}$ ceases to dissolve. If globulin is present a precipitate will form. This can be removed by filtration, washed with saturated $\mathrm{MgSO}_{4}$ solution, and finally dissolved in water (See page 47, Globulin Exp. 1). This solution of the $\mathrm{MgSO}_{4}$ precipitate can now be tested. It coagulates on heating especially if slightly acidified with acetic acid. It gives the nitric acid and heat tests, also the biuret reaction.

The filtrate from the $\mathrm{MgSO}_{4}$ precipitate contains albumin if any is present. The tests just given applied to this filtrate, if positive, prove the presence of albumin.

\section{Albumose.}

This compound, or rather group of compounds, can be readily prepared from Witte's or Schuchardt's commercial pepton since this consists largely of albumoses. Albumoses are precipitated by saturation with $\left(\mathrm{NH}_{4}\right)_{2} \mathrm{SO}_{4}$ or with $\mathrm{NaCl}$ in an acid solution.

A $20 \%$ solution of the commercial pepton is used. The powder readily dissolves especially if the liquid is warmed and thoroughly stirred.

1.-Place scme of the solution in a test-tube and heat to boiling. The liquid does not coagulate, thus indicating the absence of albumin and globulin.

2.-To 10 c.c. of the solution add 10 c.c. of saturated $\left(\mathrm{NH}_{4}\right)_{2} \mathrm{SO}_{4}$ solution and about $8 \mathrm{~g}$. of powdered $\left(\mathrm{NH}_{4}\right)_{2} \mathrm{SO}_{4}$. Saturate the liquid in a water-bath at about $30-35^{\circ}$ according to the directions given in experiment 4, page 48. Notice the sticky precipitate that adheres to the rod and to the sides of the beaker or tube. Since albumin and globulin are absent, as ascertained from preceding experiment, the precipitate that forms consists of albumoses. Transfer the precipitate to a filter and wash with about 10 c.c. of saturated $\left(\mathrm{NH}_{4}\right) \mathrm{SO}_{4}$ solution.

Save the filtrate (A) for subsequent tests for pepton. 
By means of a glass rod gather the sticky albumose precipitate and transfer it to about 20 c.c. of water in a test-tube. While stirring, heat the liquid carefully and the albumose dissolves completely.

With this aqueous solution of pure albumose make the following tests, employing small quantities of the liquid, about 1 c.c.

(a). Heat a portion to boiling. It does not coagulate.

(b). To a portion add $\mathrm{HNO}_{3}$, drop by drop. A slight precipitate may form which dissolves and gives a yellow solution. If there is no permanent precipitate add some saturated $\mathrm{NaCl}$, drop by drop, till a precipitate does form. Now gently heat the contents of the tube. The precipitate dissolves and on cooling reappears. This reaction is characteristic of albumoses.

In the absence of $\mathrm{NaCl}$ some of the albumoses, especially deuteroalbumose, do not give a precipitate with $\mathrm{HNO}_{3}$. An excess of $\mathrm{NaCl}$, however, should be avoided, since in that case the albumose precipitate does not dissolve completely on heating.

(c). To a portion of the solution add a few drops of acetic acid $(1-10)$ and $2-3$ drops of potassium ferrocyanide. If no precipitate forms add $\mathrm{NaCl}$ according to directions given above under $(b)$. A precipitate will then form and on heating gently it dissolves. On cooling the solution it reappears.

This reaction, like the preceding, is also characteristic of albumoses. A certain amount of $\mathrm{NaCl}$ is necessary as in the $\mathrm{HNO}_{3}$ test.

(d). To about 1 c.c. of the solution add 1-2 drops of dilute acetic acid and about 5 c.c. of a saturated $\mathrm{NaCl}$ solution. A precipitate or cloudiness results. On heating this disappears, to reappear on cooling.

To the remainder of the solution of albumose apply the tests given in the table (page 46) and note the result. In which of these reactions will the presence of chlorides and of ammonium salts interfere?

Apply the biuret test without the aid of heat. The hydration proteids give this reaction readily in the cold.

\section{$3 *$.-Detection of albumose in the urine.}

The reactions given above under 2 , especially $b, c$, and $d$, are characteristic of albumoses. To detect albumose in the urine, or in other liquids, it is necessary first to remove the albumin and globulin. This can be readily done by 
acidifying slightly with acetic acid and applying heat. The albumin and globulin coagulate. To the filtrate the biuret test can be applied. If the result is negative it indicates the absence of albumoses and also of pepton. If, however, the result is positive, it is due either to albumoses or to pepton, or to both. The tests given above under $2 b$, $c$, and $d$, can now be applied and if positive, the presence of albumose is demonstrated. If these tests fail the positive biuret reaction is due to pepton.

Another method for the detection of albumose consists in saturating the urine with salt, acidulating with acetic acid and boiling. The albumin and globulin coagulate; the albumose is in solution. Filter boiling hot. If the filtrate on cooling gives a precipitate it is due to albumose. To the filtrate apply the biuret reaction.

Compare methods of detection given under pepton.

\section{Pepton.}

Pepton is not precipitated by $\left(\mathrm{NH}_{4}\right)_{2} \mathrm{SO}_{4}$. The filtrate $(\mathrm{A})$ obtained in experiment 2 under albumose therefore contains pepton if it be present.

To this filtrate apply the biuret test in the cold. A positive reaction is due to pepton. As previously indicated the hydrated proteids, as a rule, do not require heat in order to give the biuret reaction.

To obtain a pure solution of the pepton it would be necessary to resort to dialysis, or to treatment with baryta on a water-bath to remove the $\left(\mathrm{NH}_{4}\right)_{2} \mathrm{SO}_{4}$.

To the original filtrate containing pepton apply the tests given in the table on page 46 and note the results. With which of these reactions will the $\left(\mathrm{NH}_{4}\right)_{2} \mathrm{SO}_{4}$ present interfere?

* Detection of pepton (Hofmeister).

To about 500 c.c. of the urine, or to an aqueous extract of the tissue to be examined, made at about $40^{\circ}$, add just enough lead acetate to give a strong precipitate and filter. 
This removes mucin. Test the filtrate for albumin and if present remove in the following manner: Add a little sodium acetate and then concentrated ferric chloride till the mixture is blood red in color. Then neutralize with potassium hydrate (or leave slightly acid), boil, cool and filter. The filtrate should give no precipitate with acetic acid and potassium ferrocyanide (absence of iron and of albumin). If it is perfectly free from albumin make the following tests:

1.-Add acetic acid and phosphotungstic acid-a cloudiness forms on standing if pepton is present.

2.-If pepton is indicated by the above trial it can be isolated by the following method: Add 0.1 volume of concentrated hydrochloric acid and then phosphotungstic acid, also acidulated with hydrochloric acid, as long as a precipitate continues to form. Filter at once and wash with dilute sulphuric acid ( 3 to 5 c.c. in 100 c.c. of water), till the filtrate is colorless. While the precipitate is still moist mix it with an excess of powdered barium hydrate, add a little water, gently warm for a short time and filter. To the filtrate which contains pepton apply the biuret test.

The Hofmeister method strictly speaking does not indicate true peptones, but rather albumose.

Another method for the detection of pepton is based upon its behavior to $\left(\mathrm{NH}_{4}\right)_{2} \mathrm{SO}_{4}$. The method as employed by Devoto is as follows; To 200-300 c.c. of the urine add $80 \%$ by weight of $\left(\mathrm{NH}_{4}\right)_{2} \mathrm{SO}_{4}$. This is added to urine even if albumin and globulin are absent in order to remove nucleoalbumin. Warm the mixture on the water-bath till the salt dissolves. This will occur in 10-15 minutes. Now place the beaker in a steam sterilizer for 30-40 minutes or longer. The albumin coagulates completely irrespective of the reaction of the fluid. The mixture is allowed to cool, then filtered. The filtrate can be tested for the biuret reaction. If positive pepton is present. It can further be precipitated 
with tannic acid. Under these conditions deutero-albumose is not completely precipitated and hence can be easily mistaken for true pepton.

The residue on the filter can be washed with hot water till the filtrate ceases to give a test for $\mathrm{BaSO}_{i}$. If the filter has previously been dried and weighed, and is now again dried and weighed the difference is due to the albumin and globulin. (See estimation of albumin and globulin).

The first portions of the hot wash-water are collected, combined and tested for the biuret reaction. If positive it is ordinarily said to be due to pepton (Devoto, Jaksch), but in reality it is due to the albumoses which are not rendered insoluble, like albumin and globulin, and dissolve on trea1ment with hot water.

The Hofmeister method it is evident will often give positive results where Devoto's method fails.

In reality the reaction in both cases as indicated above is due to albumoses. The true pepton which would be pres. ent in the filtrate from the cold saturated solution seems to be very rare, if at all, in urine.

As used in a clinical way the term "pepton" includes pepton and albumoses. Such pepton may be present, though not always, in the blood of the leukæmics during life. The blood obtained from deceased leukæmics, especially if decomposition has set in, is rich in such pepton. The normal liver does not contain pepton, whereas the spleen does. The liver and spleen of leukæmics are rich in such pepton.

A better process for the detection of true pepton is as follows:

Saturate the solution at the boiling point with ammonium sulphate and filter while boiling hot. Allow the filtrate to cool, decant the liquid from the crystals which separate, dilute strongly and precipitate the pepton by cautious addition of tannic acid. Let stand for 24 hours, then filter. 
Boil the precipitate for a few minutes with baryta water, filter, and from the filtrate remove the excess of barium by passing carbonic acid. Filter off the barium carbonate and test the filtrate for biuret.

\section{Gelatin.}

To study the reactions of gelatin a $2 \%$ solution of the best French gelatin (silver) is employed.

1.- Shake up some of the solution. Notice the foaming of the liquid.

2.- To a portion of the solution add some bromine water. An abundant, yellow, sticky precipitate forms.

3.-In each of two test-tubes add 1-2 drops of saturated $\mathrm{HgCl}_{2}$ solution. To tube 1 add about 5 c.c. of the gelatin solution. To tube 2 add about 5 c.c. of water. Then add to each tube some $\mathrm{H}_{2} \mathrm{~S}$ water and heat. Tube 1 is dark yellow, but contains no precipitate, whereas tube 2 has a blackish precipitate of $\mathrm{HgS}$ and the liquid is clear. Gelatin prevents the precipitation of many, otherwise insoluble, compounds. Glycogen possesses a similar property.

4.-To the gelatin solution apply the several tests given in the table on page 46 . Tabulate the results, and carefully note the differences.

Observe that the heavy metals do not precipitate gelatin, whereas the other proteids are precipitated. Also, that gelatin is not precipitated by ferrocyanide even in the presence of $\mathrm{NaCl}$, and in this respect it resembles pepton.

The xanthoproteic reaction is weak owing to the absence of the phenol group, $\mathrm{C}_{6} \mathrm{H}_{5} \mathrm{OH}$. The biuret reaction applied to the cold solution of gelatin gives a bluish violet color, whereas pepton gives a purple red. Millon's reagent gives a white precipitate which on heating becomes red, and the liquid becomes pink. It is probable that the other reactions are not strictly due to the gelatin but to an admixture of some pepton or albumose.

Whereas albumin contains three aromatic groups (see page 40) gelatin contains but one, since it yields phenyl amidopropionic acid or tyrosin on decomposition. 


\section{CHAPTER IV.}

\section{SALIVA.}

Saliva is a mixture of the secretions of the parotid, submaxillary, and sub-lingual glands. The reaction of mixed saliva is usually alkaline but may on fasting, also during the night toward morning, and 2-3 hours after meals, or after much talking, become acid. It also becomes acid on standing a few hours (Repin). It is more or less opalescent and viscid and foams readily. The character of the saliva will vary according to which gland furnishes the most of the secretion. The parotid gland yields a fluid secretion, whereas the submaxillary and sub-lingual glands yield slimy secretions. In febrile diseases the secretion of saliva may be diminished or wholly suppressed, and hence dryness of the mouth and throat, as well as altered taste. A decrease is also observed in diabetes, in severe diarrhœas, as in cholera. The administration of potassium iodide or of mercury produces an increased flow, or salivation, and the composition of the saliva itself becomes altered. Albumin is then present and the amount of salts in solution is increased. An increased flow of saliva (ptyalism) is also brought about by irritant poisons such as acids and alkalis; also by certain foods, lemon, etc., and occurs also in some diseases, especially in inflammatory conditions of the mouth, tonsils, and palate.

In icteric conditions the saliva does not contain bile constituents. In diabetes it does not contain sugar. In the latter case, however, the action may be acid because of lactic acid. In nephritis, urea may be present in the saliva, and uric acid has been found in uræmic conditions. Leucin has been found in the saliva of a hysteric case. 
Salivary calculi which are occasionally deposited in the salivary ducts consist chiefly of calcium carbonate and phosphate, cemented with organic matter. The tartar deposited on teeth has essentially the same composition, the phosphates however predominate. These calcium salts are held in solution in the saliva by carbonic acid. On exposure to the air this passes off and the salts are deposited.

The specific gravity of the mixed saliva varies from 1.002 to 1.008 . Such saliva contains $1 / 2-1 \%$ of solids which consist of albumin, mucin, ptyalin, traces of urea and other nitrogen compounds and mineral constituents. The amount of saliva secreted in the course of 24 hours is $1400-1500$ c.c. The flow is increased after meals and by pilocarpin. Atropin diminishes salivary secretion.

The chemical examination of saliva has at present but little clinical significance. Physiologically, however, the composition and action of saliva is of the greatest importance. The ferment or enzyme present in the saliva is known as ptyalin and possesses a diastatic or amylolytic action. That is, it converts starch into dextrin, then into iso-maltose and maltose. Eventually glucose forms probably, however, the result of the action of an inverting ferment. Ptyalin is not present in the saliva of all animals. The parotid saliva of new-born contains ptyalin, whereas the submaxillary saliva does not contain it for several months. In the saliva of some animals as the horse the ferment is not present in the free state but as a zymogen from which it readily forms in mastication. This, as well as the other enzymes, is dragged down mechanically by a precipitate of calcium phosphate and this fact is utilized to obtain the ferment in a comparatively pure state.

Although ptyalin resembles in its action the diastase of malt, it is different. This is seen in the fact that the former acts best at $40^{\circ}$, the latter at $50-60^{\circ}$. The amount of ptyalin present in the saliva is subject to variation. $\mathrm{HCl}$ not only prevents the action but it also destroys the 
ferment. The action of the ptyalin is most marked in neutral or very faintly acid saliva.

A microscopic examination of the saliva will always show epithelial cells from the mouth and tongue, also salivary and mucous corpuscles. Bacteria are always numerous, and certain species as the leptothrix, spirillum, and spirochæte are almost invariably present. Among the pathogenic forms found in the mouth in health or in disease may be mentioned the bacilli of diphtheria, tuberculosis and tetanus, Fraenkel's diplococcus, the micrococcus tetragenus and the pus-producing staphylococci and streptococci, the fungus of thrush and of actinomycosis. Blood or pus cells may be present in the saliva in inflammatory or suppurative conditions of the mouth, gums, etc.

Microscopic Examination.-Rub the tongue thoroughly over the inside of the mouth, teeth and gums, collect the saliva and examine under the microscope for epithelial cells, salivary corpuscles, etc.

The saliva necessary for the following experiments can be readily obtained by chewing a piece of pure paraffin. Commercial gum must not be used inasmuch as it contains sugar. Collect about 100 c.c. of saliva.

1. - Test the reaction of the mixed saliva with litmus paper. What is it?

2.-Nearly fill a 50 c.c. graduate with saliva. If there is any foam on the surface remove it with a piece of filter paper. Then immerse an urinometer and note the specific gravity of mixed saliva. What is the reading if immersed in pure water?

3. - To about 5 c.c. of saliva add a few drops of acetic acid (1-10) and gently agitate. A flocculent precipitate of mucin forms.

4.-To some saliva apply the biuret test (Exp. 1, p. 39). The result is due to mucin.

5.- To some saliva add a drop of nitric acid and boil. Is albumin present in saliva? 
6.-To the contents of the tube from the preceding experiment add $\mathrm{NH}_{4} \mathrm{OH}$. An orange yellow solution forms-xanthoproteic reaction.

7.--To some saliva add a few drops of Millon's reagent. A heavy yellowish precipitate forms, which on boiling becomes reddish. This is due to mucin.

8.- To some of the saliva add a drop of dilute $\mathrm{HCl}$, then, drop by drop, dilute ferric chloride till a red coloration results. This is due to the formation of ferric sulphocyanate. The reaction is more distinct if after the addition of $\mathrm{HCl}$ the liquid is filtered and the ferric chloride is added to the filtrate.

9. - To another portion of saliva add a little iodic acid and some starch solution. Iodine is liberated and colors the starch blue. This is due to a sulphocyanate. Explain the reaction.

10. - To some saliva add a few drops of dilute $\mathrm{H}_{2} \mathrm{SO}_{4}$, mix; then add a few drops of a colorless solution of potassium iodide and finally a few drops of starch solution. Iodine is liberated and colors the starch blue. This is due to nitrous acid. Explain.

11. - To some saliva add a drop or two of dilute $\mathrm{HCl}$, then $2-3$ drops of a saturated sulphanilic acid solution and mix. Now add a few drops of naphthylamine hydrochloride. A pink or red solution indicates the presence of nitrous acid. This test is employed in testing for nitrites in water analysis.

12.-Take a small dose of potassium iodide, rinse out the mouth thoroughly with water, and test some of the saliva at once for KI. This is done by adding to some of the saliva a little chlorine water, and then shaking with carbon bi-sulphide. A pink coloration of the latter indicates iodine. Iodine should be absent from the saliva after rinsing. After that collect a little of the saliva every 15 minutes, and test for iodine as above. How soon does KI appear in the saliva after being taken into the stomach?

13.- Separation of Mucin.-Pour 10 c.c. of the saliva slowly and with constant stirring, into 50 c.c. of absolute alcohol. A fibrinous light precipitate forms. Allow to settle over night in a covered beaker. Then filter, wash the precipitate on the filter twice with alcohol, then with ether. Spread out the filter to dry and finally with a spatula remove the white chalky powder of mucin.

(a). To a little of the powdered mucin in a tube add some water. It swells up, but does not pass into solution. Then add a drop or two 
of $\mathrm{KOH}$ when it dissolves, forming a milky solution. To this solution now apply the biuret test. What is the result?

(b). Place the remainder of the powder in a tube and add dilute $\mathrm{HCl}(1-3)$ and boil for some minutes. Transfer a portion to another tube, cool, render alkaline with $\mathrm{KOH}$ and boil with Fehling's solution. The formation of red cuprous oxide indicates the presence of a reducing substance. If this test is not given, boil again the remaining original liquid, and again test a portion as above.

Mucin is a complex proteid substance and on decomposition, as above, it yields a reducing compound which, however, is not sugar. What other substances on heating with an acid yield reducing substances?

14.-Action of Ptyalin.-Prepare a starch solution according to the directions given under starch (p. 28, Exp. 3). Into each of eight tubes place about 3 c.c. of Fehling's solution. Into each of another set of eight tubes place $1-2$ drops of dilute iodine solution.

(a). To 30 c.c. of the starch solution in a graduate add six drops of saliva, and at once mix thoroughly. Immediately after mixing pour 2-3 c.c. of the mixture into one of the tubes containing Fehling's solution, and also a portion into a tube containing the iodine. The latter colors deep blue-due to starch. Boil the tube with Fehling's solution. No reaction should take place-absence of sugar.

At intervals of two minutes apply the test with Fehling's solution and with iodine to the mixture in the manner just giren. Tabulate your results, noting the time when sugar appears in the mixture; when erythro-dextrin and achroo-dextrin appear. The time of appearance of the latter is spoken of as the achromic point. When this is reached boil some of the starch mixture with Barfoed's reagent. What is the result? What does this indicate?

At the conclusion of this experiment add to each of the iodine tubes 5c.c. of water. The characteristic color of the starch and of the several dextrins will be more apparent. Complete conversion should take place in about 15 minutes. If it does not, repeat the experiment using a larger amount of saliva.

(b). To 10 c.c. of starch solution add 5 c.c. of saliva, mix and make tests as rapidly as possible with iodine and with Fehling's solution. What is the result?

(c). Boil 5 c.c. of saliva in a tube for $1-2$ minutes, then add 10 c.c. of the starch solution and mix. Immediately test a portion as 
above and also at the end of $\mathbf{1 5}$ minntes. What is the result? What is the action of heat on ptyalin?

(d). To 10 c.c. of starch solution add 0.2 c.c. of a $1 \%$ acetic acid solution, mix and then add two drops of saliva. 'Test immediately with iodine and with Fehling's solution, and also at the end of 5, 10, and 15 minutes. The mixture contains about $0.02 \%$ acetic acid. What is the effect of this amount of acetic acid on the rate of inversion?

(e). To 10 c.c. of the starch solution add 0.6 c.c. of a dilute $\mathrm{HCl}$ $(0.3 \%)$. The latter is prepared by adding 8 c.c. of the concentrated acid to one litre of water. Mix, then add two drops of saliva and again mix thoroughly. This mixture now contains about $0.02 \%$ $\mathrm{HCl}$, about the same degree of acidity as in the preceding experiment, and about one-tenth of that of the gastric juice. Test the mixture at once with iodine and with Fehling's solution, and also at the end of 5, 10, and 15 minutes. Note carefully the result. How does the action of $\mathrm{HCl}$ compare with that of acetic acid. 


\section{- CHAPTER V.}

GASTRIC JUICE.

The sastric juice is the combined product of several glands, cardiac and pyloric, and normally possesses an intense acid reaction due to the presence of free hydrochloric acid. In certain diseases as a result of fermentative changes the acidity may be in part, or wholly, due to organic acids'such as lactic, acetic, butyric, etc. The material which is obtained from the stomach after a test breakfast, by means of lavage, usually contains besides remnants of food, more or less mucus. Before it is employed for tests it should therefore be filtered. The gastric juice proper is a watery fluid which filters easily and is not slimy. Its specific gravity varies from 1,002 to 1,010 .

The contents of the stomach may contain: (1) Microscopical constituents, such as remains of food; squamous epithelial cells, rarely columnar; blood cells; various microorganisms, such as bacilli, micrococci, rarely spirals; sarcines, yeasts and leptothrix threads are common. (2) Soluble chemical constituents.

The latter are of first importance physiologically. They include the proteolytic enzyme, pepsin; the milkcurdling ferment, rennin or chymosin; hydrochloric acid present either in free state, or loosely combined, or as ordinary chloride; organic acids, such as lactic acid, etc., resulting from bacterial fermentation; lastly acid phosphates, peptons, etc.

The secretion from the pyloric end of the stomach is usually said to be alkaline and to contain only pepsin. On the other hand, the secretion from the cardiac end is intensely acid and contains pepsin. 
The hydrochloric acid is unquestionably derived from the sodium chloride of the blood. As a result of mass action the large amount of carbon dioxide present in the blood reacts upon the sodium chloride. Dissociation results and a minute amount of free hydrochloric acid is present in the blood. Certain cells of the stomach by virtue of their selective power transmit this free acid to the gastric juice.

The amount of free hydrochloric acid present in the gastric secretion varies considerably, but in general is said to average about 0.2 per cent. On contact with albuminous substances the acid unites with these and gives rise to what is known as "loosely-combined hydrochloric acid." When in this combination the protein molecule is prepared for the action of the ferment pepsin. Consequently the loosely combined hydrochloric acid may be considered as the physiologically active acid. Furthermore, the hydrochloric acid probably unites with pepsin to form the socalled "pepsin-hydrochloric acid."

Hydrochloric acid is an effective germicide and, moreover, stops the diastatic action of ptyalin on starch. It does not follow, however, that the salivary digestion of starch ceases the moment the food reaches the stomach, or that all bacteria are destroyed in the stomach. With reference to starch conversion it may be said that this will continue until free hydrochloric acid has permeated the entire mass of food contained in the stomach. If the mass of food is large, or if the amount of hydrochloric acid secreted is small, this diastatic action may continue for a considerable time after the food has been taken into the stomach.

As to the germicidal action of the hydrochloric acid contained in the gastric juice it should be remembered that it is exerted against the vegetative form of bacteria and not against spores. Moreover, since ptyalin may continue to act on starch in the stomach for some time owing to the fact that the acid secreted must first neutralize basic con- 
stituents, unite with proteins, and finally permeate the entire mass as free acid, it follows that many bacteria introduced with the food may not be exposed to the free acid for a considerable period of time. Portions of the stomach contents are passed into the intestines at frequent intervals, and hence even weak, vegetative forms, such as the cholera vibrio may pass the stomach uninjured. It is not necessary to neutralize the stomach contents in man and in animals in order to produce experimental cholera infection.

An increased secretion of hydrochloric acid (hyper. acidity) results in more rapid solution of proteins, and in early inhibition of the diastatic action of saliva on starch. Such a stomach fluid though rich in free acid, if it remains in the stomach for some time, undergoes bacterial decomposition. Organic acids, gases, as marsh-gas, hydrogen, carbon dioxide, etc., form, but putrefaction of the albuminous substances does not take place.

Hydrochloric acid is frequently diminished in amount (hypo- or sub-acidity). This decrease may be considerable, but the total absence of free hydrochloric acid is rare. Such a decrease is met with commonly in dyspepsia, in cancer of the stomach, in febrile diseases, in cirrhosis of the liver and frequently in nephritis.

With the decrease in the amount of free acid present, naturally its inhibiting influence on bacteria is diminished, consequently bacterial growth in the stomach may become enormously developed. In such instances even the characteristic mouth bacteria as leptothrix, spirochætes, comma bacilli, etc., may develop in the stomach contents. As a result then, of a decrease in free hydrochloric acid, various fermentations may arise. At one time it may be lactic acid, at another time acetic or butyric acid, etc., that is especially being elaborated. The longer the food remains in the stomach, because of motor insufficiency, the more marked will such decomposition be.

A stomach will undoubtedly disinfect itself if a suffi- 
cient interval is allowed between meals. These intervals should be not less than 10 or 12 hours.

The enzymes, pepsin and rennin, are secreted by the stomach even in severe diseases, and it is only in general atrophy of the mucous membrane of the stomach that they are said to be absent. It is doubtful, however, if these enzymes are ever wholly absent from the gastric secretion. Pepsin, as well as a diastatic ferment, appear in the urine in starvation. These ferments are frequently increased in the urine in fevers, as typhoid fever, tuberculosis, pneumonia, etc., and are markedly increased in diabetes. In testing urine for enzymes the utmost care must be taken to eliminate the action of bacteria.

Pepsin is present in the gastric secretion of all vertebrates. It acts on proteins in acid, but not in neutral or alkaline solution. It has no action on fats, or on carbohydrates. There is reason to believe that the pepsin of one animal is different from that of another. In other words, that there is a group of pepsins, or proteolytic ferments just as there is a group of nucleins, of hæmoglobins, etc. Similar proteolytic ferments are met with in plants (papayotin, etc). Pepsin, when isolated in the purest condition possible, does not give most of the protein reactions. When impure it is soluble in water and in glycerin. It is precipitated by alcohol, though this precipitation is to be considered largely as a mechanical dragging down of the enzymes, as in similar precipitation of bacterial toxins. Pepsin, when in solution, is readily destroyed by heat, even at $55-60^{\circ}$; whereas when dry it can be heated to above $100^{\circ}$ without change.

The characteristic action of pepsin is its digestion of proteins in acid solutions. Nucleoproteids on digestion with pepsin leave a residue of nuclein (or pseudo-nuclein in the case of certain nucleo-albumins such as casein). Even fibrin leaves a residue of nuclein on digestion which has been called dyspepton. 
The albuminous substances are first brought into solution by the free hydrochloric acid. If this solution is neutralized a precipitate of syntonin or acid albumin forms. As a result of the action of the ferment the protein is converted into albumose. It is only when peptic digestion is protracted, as for some days, that the albumoses in turn are changed into peptons. In peptic digestion the cleavage does not go below the pepton stage, whereas in pancreatic digestion a portion of the pepton formed may be split up into leucin, tyrosin, etc.

The albumoses and peptons are to be considered as hydration products of proteins, corresponding to the dextrins and to glucose in the hydration of starches. Just as the hydration of starches, fats, etc., may result from the action of enzymes, or on heating with acids or alkalis, or by bacterial ferments, so the hydration of proteins may be accomplished by these same agencies. As a result of the action of bacteria, especially of the liquefying type, albumin or fibrin may be changed to albumose, or even to pepton. The bacterial proteids probably owe their poisonous properties to a mere admixture of the real toxin. Poisonous proteids (abrin, ricin) have been isolated from plants, and also from the venoms of serpents.

The first product of the hydration of proteins is an albumose, the final product is pepton. Strictly speaking there is not merely one albumose, but a group of albumoses. The following four types of albumose are dis. tinguished:

Hetero-albumose is insoluble in water, but soluble in dilute sodium chloride. Proto-albumose is soluble in water and in sodium chloride. Both of these are precipitated by sodium chloride in neutral solution, though incompletely. They are sometimes designated as primary albumoses. As a result of the action of salts, etc., hetero-albumose may become insoluble and is then known as rys-albumose. 
Deutero-culbumose is soluble in water and in dilute sodium chloride, but is not precipitated from neutral solution on saturation with sodium chloride. It is, however, precipitated incompletely out of acid solution. This albumose is closely related to pepton and is sometimes spoken of as secondary albumose.

Not only are there several albumoses resulting from the digestion of a given protein, but when these are com. pared with similar products formed from the digestion of other proteins, certain differences are manifest. It is customary to designate the albumoses formed from globulin, vitellin, casein, myosin, as globulinose, vitellose, caseose, myosinose, respectively. The word proteose is used as a general term, covering all albumoses whether derived from animal or vegetable protein matter.

Pepton is very hygroscopic and very soluble in water. It diffuses more readily than does albumose. It is not precipitated by picric acid or by potassium mercuric iodide; and is incompletely precipitated by phosphotungstic and by phosphomolybdic acids. It is precipitated by tannic acid, but the precipitate is soluble in excess.

The protein molecule on hydration yields two groups of compounds, one of which is more resistant than the other. The more resistant product is designated as anti-albumose, anti-pepton, whereas the less resistant product is termed hemi-albumose, hemi-pepton. The gastric juice when allowed to act for some days yields a mixture of anti- and hemi-pepton. This mixture is known as ampho-pepton If this is subjected to a more energetic ferment than pepsin, namely trypsin, the hemi-pepton is broken down into leucin and tyrosin, whereas anti-pepton remains constituting one-half of the original mixed peptons.

It should not be inferred from the preceding statement that all the hemi-pepton, under the influence of trypsin in the intestine, is broken down into leucin and tyrosin. It 
does undergo this decomposition in vitro, but it does not follow from this that the same complete cleavage results in the intestines. The fact that leucin and tyrosin are present in the intestinal contents in minimal amounts, and the further important fact that anti-pepton alone will not support life, indicate that hemi-pepton is absorbed and utilized. The hemi- and anti-peptons are regenerated to albumin, etc.

Proteins $\left\{\begin{array}{l}\text { Anti-albumose-Anti-pepton } \\ \text { Hemi-albumose-Hemi-pepton }\end{array}\right\} \begin{aligned} & \text { Ampho-pepton. } \\ & \ldots . . . . . . . . .(\text { (leucin, tyrosin). }\end{aligned}$

Antipepton possesses the formula $\mathrm{C}_{10} \mathrm{H}_{15} \mathrm{~N}_{3} \mathrm{O}_{5}$, and is identified with sarkinic acid, derived from phosphosarkinic acid of the muscle. It gives a strong biuret reaction in the cold, but does not give Millon's test.

When a hæmorrhage into the stomach takes place the hæmoglobin is acted upon by the gastric juice and is split into hæmatin and globulin. The former is the cause of the brown, or coffee color, of the stomach contents.

The milk curdling ferment, known as rennin or chymosin, is apparently a constant constituent of the gastric juice of vertebrates. It is especially abundant in the mucous membrane of the stomach of the calf (rennet), and this is used to curdle milk in the manufacture of cheese. It may be absent in cancer and in chronic catarrh of the stomach, and in atrophy of the mucous membrane of the stomach. When obtained in the purest condition possible it does not give the ordinary protein reactions. It is destroyed by 0.3 per cent. $\mathrm{HCl}$ in the presence of pepsin. It coagulates milk or solutions of casein containing lime salts. Calcium is necessary for the curdling of milk and for the coagulation of blood. In the latter instance the calcium is necessary in order to make fibrin ferment; in the former case it unites with the altered casein to make para-casein. 


\section{Recognition of Free Hydrochloric Acid.}

Three solutions of dilute HCl labelled 1, 2, and 3, will be found on the side table. Solution 1 approximates in strength that found in the gastric juice. It is prepared by diluting 6 c.c. of $\mathrm{HCl}$ (1.19 specific gravity) to one liter $(=0.25 \%)$. Solution 2 is prepared by diluting 200 c.c. of solution 1 to one liter $(=0.05 \%)$. Solution 3 is prepared by diluting 200 c.c. of solution 2 to one liter ( $=0.01 \%$ ).

A $2 \%$ pepton and a $1 \%$ lactic acid solution will also be found on the side table. If the pepton solution is slightly alkaline it should be faintly acidified with acetic or lactic acid.

The following tests are given in the order of their delicacy:

1.-Di-methyl-amido-azobenzol.-This reagent is used in a $0.5 \%$ alcoholic solution. Add 3-4 drops of the reagent to some of the solution to be examined. If a pink red color forms a free mineral acid is present. In the case of gastric juice it is $\mathrm{HCl}$. A yellow color indicates an absence of HCl. Certain substances, such as pepton and organic acids, tend to interfere in this as well as in the subsequent tests. Organic acids, if concentrated, may give a somewhat similar reaction.

Note the results obtained with solutions 1,2 , and 3 , in the first column. Then mix the same amount of these solutions with an equal volume of $2 \%$ pepton, and to this mixture apply the test and note the results in the second column. In the same way make a mixture of the three solutions with an equal volume of a $1 \%$ solution of lactic acid, test and note the results.

\begin{tabular}{|c|c|c|c|}
\hline & Pure $\mathrm{HCl}$. & $\mid \begin{array}{l}\mathrm{HCl} \text { and } \\
2 \% \text { Pepton àả. }\end{array}$ & $\begin{array}{c}\mathrm{HCl} \text { and } \\
\text { 1\% Lactic Acid āā. }\end{array}$ \\
\hline 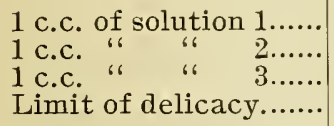 & & & \\
\hline
\end{tabular}

Apply the test to the solution of pepton, also to the solution of lactic acid. Report the results.

2.-Günzburg's reaction, or the phloroglucin vanillin test.-The reagent is prepared by dissolving $1 \mathrm{~g}$. of vanillin and $2 \mathrm{~g}$. of phloroglucin in 100 c.c. of alcohol. 
Place the solution to be tested in an evaporating dish, add $2-3$ drops of the reagent and carefully evaporate over a small flame to dryness. A purple or pinkish-red color indicates free $\mathrm{HCl}$. This has long been considered the most delıcate test for free $\mathrm{HCl}$.

Apply this test to solutions 1,2 and 3 , and to mixtures as indicated in the table given under experiment 1 . Carefully note the limit of delicacy of the reaction under the several conditions. Tabulate the results as above.

3.-Bocts reagent.-This is prepared by dissolving $10 \mathrm{~g}$. of resorcin, $3 \mathrm{~g}$. of cane sugar and $3 \mathrm{c.c}$. of alcohol in 100 c.c. of water. Place in an evaporating dish the solution to be tested, add $2-3$ drops of the reagent, and evaporate over a small flame to dryness. If a free mineral acid is present a rose or pink-red color develops and gradually fades on cooling.

Apply this test to solutions 1, 2 and 3 , and to mixtures as indicated in the table given in experiment 1 . Note the limit of delicacy and tabulate the results.

4.-Troporolin UO.-A solution of this reagent is prepared by dissolving $0.25 \mathrm{~g}$. of the reagent in $1.000 \mathrm{c} . \mathrm{c}$. of water. Instead of the solution tropaolin papers may be employed. They are, howerer, not so reliable as the solution. since with distilled water they sometimes give a pink color. To some of the acid solution add a drop of the reagent (or immerse a strip of the tropæolin paper). A pink color is due to free mineral acid. If the solution is eraporated carefully to dryness a bluish residue remains.

Apply this test to the solutions as given in experiment 1, and tabulate the results.

5.-Congo-red papers. - Tne color of these papers is changed on contact with mineral acids to a deep blue, whereas organic acids yield a violet. Immerse a strip of the paper in 1 c.c. of the solutions 1, 2 and 3, also lactic acid and distilled water, and report the results and the delicacy.

6.-Denzopupurin 6 B' paper.-These papers are turned to an intense dark brown color by mineral acids. With strips of this paper make similar tests as those given in experiment 5, and report the results.

7.-Wethyl virlet.-A solution of this reagent is prepared by dissolving $0.5 \mathrm{~g}$. in $1,000 \mathrm{c}$.c. of water. To the solution to be tested add 1-2 drops of the reagent. Free $\mathrm{HCl}$ gives a copper-blue color. 
Organic acids yield a violet blue. Apply this test, first to some distilled water, and note the color. Finally apply the test to the solutions 1, 2 and 3, and compare the results. Also test pepton and lactic acid mixtures and tabulate the results as under experiment 1 .

\section{Detection of Lactic Acid.}

Uffelmann's test.-The reagent is prepared by adding a drop of dilute ferric chloride to 10 c.c. of a $2.5 \%$ carbolic acid solution. The liquid is colored blue. This color is completely discharged by mineral acids, leaving a colorless solution, whereas organic acids discharge the color and leave a straw yellow solution. A $1 \%$ lactic acid solution is used.

1.-In each of three test-tubes place 5 c.c. of the reagent, then add to each about $1 / 2$ c.c. of the lactic acid solution. The blue is replaced by a straw yellow color.

To each of these tubes now add respectively an equal volume of the HCl solutions 1, 2 and 3 . Note the interference, if any, in the lactic acid reaction by the presence of variable amounts of free $\mathrm{HCl}$.

2.-In each of three tubes place 5 c.c. of the reagent, then add respectively an equal volume of the $\mathrm{HCl}$ solutions 1,2 and 3 . Compare the results with those obtained above when lactic acid is present.

3.- In each of six tubes place 5 c.c. of an almost colorless solution of $\mathrm{FeCl}_{3}$. To tube 1 add 1 c.c. of the $\mathrm{HCl}$ solution 1 . To tube 2 add 1 c.c. of the lactic acid solution. To tube 3 add 1 c.c. of the $2 \%$ pepton solution. To tube 4 add 1 c.c. of alcohol. To tube 5 add 1 c.c. of a $4 \%$ solution of cane sugar. Tube 6 remains blank and serves for a control. Carefully note the results.

It is evident from the above experiments that this test for lactic acid is not characteristic. In the first place free $\mathrm{HCl}$ if present in sufficient amount may interfere; and sec. ondly, a similar test is given by a number of substances which may at times be present in the stomach contents. In order to obtain a positive test for lactic acid it is necessary to isolate the lactic acid from the liquid by extraction with ether. The liquid must be extracted several times 
with ether. This is then distilled off, the residue dissolved in water and tested as above.

\section{Peptic Digestion.}

The following solutions will be found on the side table:

1.-A $0.25 \%$ solution of $\mathrm{HCl}$.- This solution is the same as solution 1 , used in connection with the tests for free $\mathrm{HCl}$. It corresponds to the normal acidity of the gastric juice.

2.-A solution of pepsin in wuter.-This is prepared by dissolving

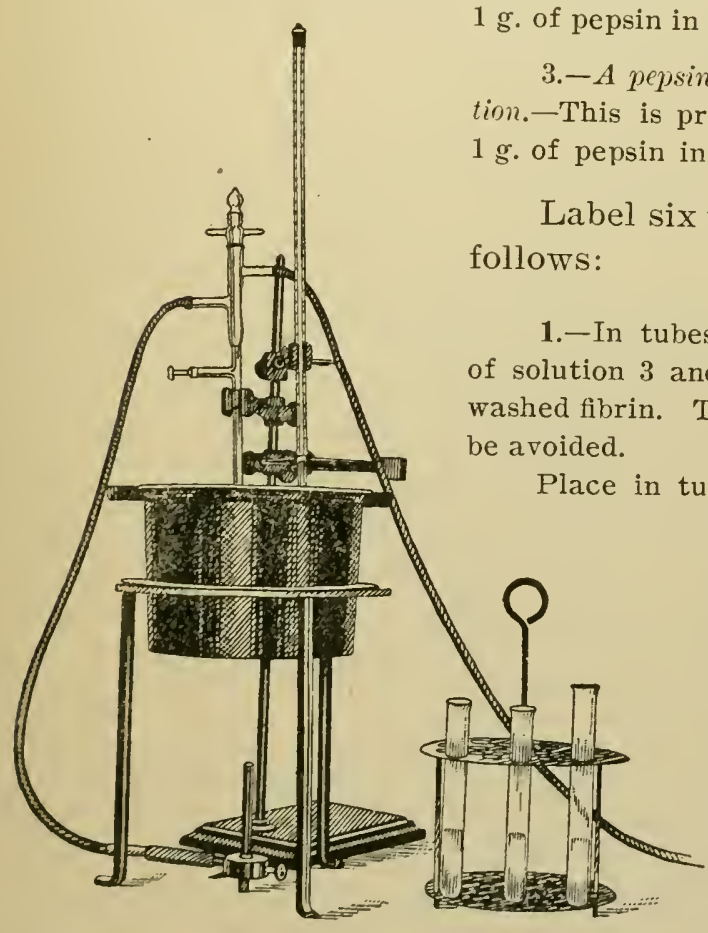

Fig. 2.

3.-A pepsin-hydrochloric acid solution.-This is prepared by dissolving $1 \mathrm{~g}$. of pepsin in 1 liter of solution 1 .

Label six tubes and equip as

1.-In tubes 1 and 2 place 20 c.c. of solution 3 and about $2 \mathrm{~g}$. of fresh, washed fibrin. Too much fibrin should

Place in tube 3,10 c.c. of solution 3. Immerse in boiling water for about 2-3 minutes; then cool to $40^{\circ}$, and add $2 \mathrm{~g}$. of fibrin.

Place in tube 4, 10 c.c. of solution 1 and about $2 \mathrm{~g}$. of fibrin.

Place in tube 5, 10 c.c. of solution 2 and about $2 \mathrm{~g}$. of fibrin.

Place in tube $6,2.5$ c.c. of solution 3 and 7.5 c.c. of solution 1 . Then add about $2 \mathrm{~g}$. of fibrin.

The tubes thus prepared are placed in an incubator at $40^{\circ}$ or $\underset{8}{i m m e r s e d ~ i n ~ a ~ w a t e r-b a t h ~ h a v i n g ~ t h a t ~ t e m p e r a t u r e ~(F i g . ~ 2) . ~ A t ~ t h e ~}$ 
end of 15 minutes the tubes are taken out and examined. Observe that in all the tubes, except tube 5, the fibrin has swelled up so that the contents of the tube are solid. Return the tubes to the incubator or water-bath and examine at the end of every hour for the next three hours. Observe the change that takes place in tubes 1 and 2 , and compare carefully with tubes 3,4 and 5 , and with tube 6 .

The tubes remain in the incubator till next day. If, however, tube 1 is completely digested in 2-3 hours, it should be treated at once according to experiment 2 . Next day carefully examine and note the condition of each tube. In tubes 1 and 2 , and possibly in tube 6 , the fibrin has disappeared. A finely granular, whitish or brownish sediment is left. What is it? Tubes 3 and 4 are about alike. The fibrin is gradually being dissolved by the dilute acid. The pepsin added to tube 3 evidently has been destroyed by boiling. No change in tube 5 .

Return tubes 2, 3 and 4, to the incubator, and keep there for 3-4 days longer.

2.-Filter the contents of tube 1 . Save a portion of the filtrate for Exp. B.

(A). To 10 c.c. of the filtrate, in a small beaker, or in a wide testtube on foot, add $8 \mathrm{~g}$. of powdered $\left(\mathrm{NH}_{4}\right)_{2} \mathrm{SO}_{4}$. Immerse for $\mathrm{t} / 2-1$ hour in a water-bath at a temperature of $30-35^{\circ}$. Stir frequently with a rod to bring the salt into solution. When the salt ceases to dissolve, $i . e$, when the liquid is saturated, the albumose present will be thrown out of solution as coarse floccules which rise to the surface forming a sticky or slimy layer. Transfer the liquid to a filter previously moistened with a little saturated $\left(\mathrm{NH}_{4}\right)_{2} \mathrm{SO}_{4}$ solution. Wash the residue with 10 c.c. of saturated $\left(\mathrm{NH}_{4}\right)_{2} \mathrm{SO}_{4}$ solution.

(a). The clear $\left(\mathrm{NH}_{4}\right)_{2} \mathrm{SO}_{4}$ filtrate contains pepton. Test this solution as follows:

1.-To a little of the liquid add an equal volume of strong $\mathrm{NaOH}$, then 1-2 drops of very dilute $\mathrm{CuSO}_{4}$ solution. A pink color results. The biuret test is given in the cold by the hydrated proteids.

2.-To another portion of the filtrate add 1-2 drops of a fresh tannic acid solution. Avoid an excess of reagent. A heavy white precipitate forms.

3.--To a portion add one to two drops of dilute acetic acid, then a drop or two of potassium ferrocyanide. What does the absence of a precipitate mean? 
(b). The $\left(\mathrm{NH}_{4}\right)_{2} \mathrm{SO}_{4}$ precipitate left on the filter is albumose. Transfer to a tube, add distilled water, warm gently and stir with a rod till dissolved. Test this solution as follows:

1.-Boil the solution. Absence of coagulation shows absence of albumin and globulin.

2. - To a portion apply the $\mathrm{HNO}_{3}$ and heat test for albumin (p. 42).

3.-To another portion apply the acetic acid and potassium ferrocyanide test for albumin (Exp. 10, p. 43).

(B). With the reserved portion of the filtrate from tube 1 make the following tests:

1.-Heat a portion to boiling. What does the absence of coagulation mean?

2. -To a little of the liquid (1 c.c.) apply the biuret test as given above under $a-1$ (p. 72).

Exactly neutralize the remainder of the solution with dilute $\mathrm{NaOH}$ and test for albumoses as follows:

3.-To a portion apply the $\mathrm{HNO}_{3}$ and heat test for albumose (p. $50, \operatorname{Exp} .2$ b).

4.-To the yellowish liquid obtained in preceding test (B 3 ) add an excess of $\mathrm{NH}_{4} \mathrm{OH}$. An orange yellow color results-the xanthoproteic reaction.

5.--To a portion add $\mathbf{1 - 2}$ drops of dilute acetic acid and a drop or two of ferrocyanide solution. If no precipitate forms add some $\mathrm{NaCl}$ solution according to directions given on p. 50, (Exp. $2 \mathrm{c}$ ).

6. - To another portion of the liquid add 1-2 drops of fresh tannic acid solution. Heavy white precipitate.

3.--After tube 2 has been kept for $3-5$ days at $40^{\circ}$, filter the contents. Saturate 10 c.c. of the liquid with $\left(\mathrm{NH}_{4}\right)_{2} \mathrm{SO}_{4}$ according to the directions given above ( $2 \mathrm{~A}, \mathrm{p} .72$ ). The liquid is cloudy, but very little albumose is precipitated? Why?

Filter the saturated liquid and to a portion of the filtrate apply the biuret test as given above under $a-1$ ( $p .72)$.

4.-The fibrin in tubes 3 and 4 , in a few days at $40^{\circ}$, is completely dissolved by the acid present. When this occurs unite the contents 
of the two tubes and filter. Exactly neutralize the filtrate according to directions given under Exp. 22, p. 46. A heavy white precipitate shows the presence of acid albumin, or as it is sometimes called syntonin. Pepton may also form but will remain in solution.

\section{Examination of Stomach Contents.}

* The stomach and contents of a recently fed rabbit (or larger animal) are cut up, diluted with about 500 c.c. of water and placed at $40^{\circ}$ for about one hour. The mixture is then filtered through muslin. This dilute gastric juice is used for the following experiments:

1.-Test the reaction with litmus paper. It is distinctly acid.

2.-Test portions of the liquid for free $\mathrm{HCl}$ according to I, 1 and $2,($ p. 68). (p. 70$)$.

3.-Test a portion for lactic acid with Uffelmann's reagent,

4. - To 10 c.c. of the solution add a shred of fibrin or a flake of coagulated egg albumin. Set aside at $40^{\circ}$ for $2-3$ hours. If not dissolved let the tube remain at this temperature over night. Then filter, and to the filtrate apply the biuret test in the cold ( $a 1$, p. 72).

5.-Apply the biuret test direct to a portion of the dilute gastric juice and compare the intensity of the reaction with that obtained in 4 .

6. -In each of three test-tubes place 10 c.c. of fresh milk. To tube 1 add 2 c.c. of the solution, previously carefully neutralized. To tube 2 add one drop of commercial rennet solution. Tube 3 serves as a control. Set the tubes aside at $40^{\circ}$ and examine occasionally during the next hour.

7.-Test for pepsin.-The following test is applicable to vomited matter, or to the liquid obtained from a stomach. Dilute 20 c.c. of the liquid, if necessary, and filter. To one-half of the filtrate in a testtube add a few shreds of washed fibrin, or a flake of coagulated egg albumin. Set aside at $40^{\circ}$ for $1 / 2-1$ hour. The fibrin should dissolve, the egg albumin requires more time. If no digestion takes place it may be due to the absence of $\mathrm{HCl}$, or of pepsin, or of both.

To the other half of the filtrate add an equal volume of $0.5 \% \mathrm{HCl}$. 
This is prepared by diluting 6 c.c. of concentrated $\mathrm{HCl}$ (1.19 specific gravity) with water to 500 c.c.

To the mixture of filtrate and acid add fibrin or egg albumin and set aside at $40^{\circ}$ as above. If in both of these tests the fibrin or albumin remains undissolved it is due to the absence of pepsin.

Each student will receive five "unknowns" and these are to be tested for lactic acid, free $\mathrm{HCl}$, pepsin, and rennet according to the directions given above under IV $1,2,3,6,7$. One or more of these may be expected in such an unknown. Report the results.

The flakes of coagulated egg albumin are best prepared by gradually pouring a dilute solution of the egg albumin, with constant stirring, into boiling water. 
CHAPTER VI.

\section{PANCREATIC SECRETION.}

The pancreatic secretion is a clear thick alkaline fluid, rich in solids and possesses very active ferment properties. It contains at least three distinct ferments, besides albumin, leucin, fats, soap and salts. These solid constituents make up about $10 \%$ of the secretion. After a pancreatic fistula has been in place for sometime the secretion is altered. It becomes thinner, strongly alkaline, and shows little or no proteolytic action. The amount of solids in this altered secretion scarcely exceeds two per cent. The quantity of the secretion given off in a period of 24 hours is not definitely known.

The ingestion of food stimulates the flow of the pancreatic fluid. There is, therefore, no secretion during starvation and it is intermittent in carnivorous animals where some time elapses between meals. On the other hand secretion is going on almost continually in herbivorous animals because digestion is uninterruptedly taking place.

As stated above the pancreatic secretion contains at least three distinct ferments or enzymes splitting up respectively fats, carbohydrates and proteids.

The neutral fat which is taken into the body with the food is acted upon by one of the ferments, steapsin or pialyn, and is split up by hydration or saponification into free fatty acids and glycerin. This ferment is very readily decomposed by acids and may be absent, therefore, from old pancreas. Only a small portion of the fat, however, undergoes this change. The free acids now combine with sodium carbonate to form soaps and the resulting soap solution readily 
emusifies the remaining neutral fat and thus brings it into a finely divided condition suitable for absorption. A considerable portion of the fat may at times be decomposed into free fatty acids through the activity of bacteria. The free fatty acids are not absorbed as such, but appear to be regenerated in the intestinal walls, by synthesis, into neutral fat. Only a very small amount of fat seems to be absorbed as soap.

The cleavage of fats by the pancreatic ferment and the subsequent emulsification is necessary to the proper absorption of fats. In addition to the pancreatic secretion, the bile plays an important part in the absorption of fat. It is well known that closure of the bile-duct, whether experimentally or in disease, as in icterus, is followed by diminished absorption and hence increased excretion of fat, more especially fatty acids, in the feces. Some fat, however, continues to be absorbed even in the absence of the bile secretion. The pancreatic secretion is necessary since no absorption of fat takes place when the pancreas is extirpated. In the latter case, however, milk continues to be absorbed owing to the already emulsified condition of the fat. Some fat may, at times, be absorbed even after total extirpation of the pancreas, since bacterial ferments may split up the fat and thus emulsification and hence absorption may result.

The second ferment of the pancreas acts on starches, splitting up the bodies into dextrin and iso-maltose. This ferment is spoken of as amylolytic or diastatic, and resembles in its action the ptyalin of the saliva. It is probably not identical with the saliva ferment. It is soluble in water and in glycerin; insoluble in alcohol. This diastatic ferment appears to be absent during the first few weeks of infant life. At the temperature of the body it acts rapidly on boiled starch, converting this into amylodextrin, erythrodextrin, achroo-dextrin, iso-maltose and maltose. By 
the action of a special inverting ferment the maltose then is converted into glucose in which form the carbohydrates are chiefly absorbed. Other mono-saccharides as lævulose and galactose may also be absorbed direct. It is possible for small amounts of dextrin and for milk sugar to reach absorption.

Sugar is absorbed very rapidly, so much so, indeed, that if a very large amount be ingested at one time it appears in the urine. This condition, known as alimentary glycosuria, does not occur when large quantities of starch are ingested. Although the pancreatic gland is necessary for the complete absorption of all the starch ingested, it is a noteworthy fact that about one-half of the starch ingested will still be absorbed after total extirpation of the pancreatic gland. This may be explained by the diastatic action possessed by many bacteria.

The third ferment of the pancreatic secretion is proteolytic in its action and is known as trypsin. This ferment does not exist as such in the substance of the gland, but is represented by a parent-substance trypsinogen, which is most abundant in the gland in from 14-18 hours after a meal. This zymogen during the process of secretion is converted into the enzyme, trypsin. Just how this takes place is not definitely known. This conversion can be accomplished artificially by the action of air, water, acids, very weak alkalis and various other substances. Stronger alkalis prevent cleavage of the zymogen. It is probable that, as in the case of pepsin, the pancreatic secretion of different animals contains slightly different trypsins.

Trypsin, like some other ferments, in its purest condition gives proteid reactions. It is soluble in water, insoluble in alcohol and in glycerin. When in an impure state, however, it may be dissolved by glycerin. This is true of the other enzymes. In neutral or slightly alkaline solution it is readily destroyed at $50^{\circ}$. It is also destroyed by gastric 
juice and unlike pepsin it digests fibrin in alkaline, neutral or even very faintly acid solutions. It is destroyed by mineral acids, but not as a rule by organic acids. The fibrin in tryptic digestion does not swell and is not irregularly eaten away as is the case in peptic digestion. The fibrin digestion with trypsin takes place most rapidly at about $40^{\circ}$, and in slightly alkaline solution $\left(0.3 \% \quad \mathrm{Na}_{2} \mathrm{CO}_{3}\right)$.

In view of the fact that trypsin acts best under the conditions mentioned, it is evident that the products of the tryptic digestion will be mixed with various bacterial products unless special attention is given toward inhibiting the growth of these micro-organisms.

In actual experiments, therefore, thymol or chloroform is added to suppress the bacteria. In the intestines, of course, during pancreatic digestion the bacteria are unhindered in their action. Among the products resulting from the action of trypsin proper on fibrin may be mentioned albumoses, pepton, leucin, tyrosin, asparaginic acid, lysin, ammonia and proteinochromogen. True pepton is formed much more readily in tryptic than in peptic digestion. This pepton, on prolonged digestion, is eventually of the kind known as anti-pepton, whereas the hemi-pepton has been decomposed yielding products such as leucin, tyrosin, etc. Trypsin dissolves gelatin yielding a gelatin-pepton. The collagens or gelatin-yielding connective tissues are not acted upon until they have been altered by heat or acids. Trypsin has no action on fats or carbohydrates.

Cut up the fresh pancreatic gland into very fine pieces, or better pass it through an Enterprise fruit-press. The pulp thus obtained can be used direct, or mixed with several volumes of water.

Place about 10 c.c. of the pulpy mixture in a small beaker, add 25 c.c. of water and boil for about 10 minutes. Crush the hard coagulated lumps in a mortar and return to the liquid. Reserve this for experiments $1 \mathrm{l}, 3 \mathrm{~b}$.

1.-Cleavage action on fats. - The fat or oil employed for this test should be strictly neutral. It can be obtained in this condition by the 
following process: Place about 10 c.c. of the oil (cotton-seed oil or butter) in a small separatory funnel, add 20 c.c. of water and render the mixture distinctly alkaline with $\mathrm{NaOH}$. Then add an equal volume of ether and shake till the fat dissolves. Draw off the aqueous liquid and to the ether add an equal volume of water, and shake again to wash the ether. Remove the aqueous layer and wash once more with water. Transfer the ether solution, filtered if need be, to a porcelain dish and allow the ether to evaporate. The neutral fat is left behind.

Place in each of two test-tubes about 3-4 c.c. of the neutral fat, 15 c.c. of water and a few drops of concentrated aqueous blue litmus solution.

(a). To one test-tube add about 5 c.c. of the fresh pancreatic pulp mixture and shake.

(b). To the second tube add one-half of the liquid containing the boiled pulp and shake. If the contents of the two tubes react acid add, drop by drop, a $\mathrm{Na}_{2} \mathrm{CO}_{3}$ solution (2\%) until the mixture is distinctly alkaline.

Place the tubes in an incubator at $40^{\circ}$ for $6-8$ hours, or less. Compare the reaction of the tubes. Reserve the two mixtures for the next experiment. If the mixtures remain too long at this temperature bacteria develop give rise to acids, and reduce the litmus. The two tubes will then be quite alike and will give the same results in the next experiment.

Under the influence of a ferment in the pancreas, known as steapsin, or pialyn, the neutral fat is, in part, decomposed into free fatty acid and glycerin.

2.-Emulsifying action on fats.-After digesting the two mixtures at $40^{\circ}$ for $6-8$ hours in the preceding experiment, shake thoroughly and take $1 / 3-1 / 2$ of the contents of each tube and treat as follows:

(a). To a portion of the mixture from Exp. $1 a$ add about 1 c.c. of $\mathrm{Na}_{2} \mathrm{CO}_{3}$ solution (2\%) and shake thoroughly. The liquid becomes milky and on standing the fat does not rise to the surface. Examine a drop of the emulsion under the microscope.

(b). To a portion of the mixture from Exp. 16 add $\mathrm{Na}_{2} \mathrm{CO}_{3}$ solution as above and shake. The liquid does not emulsify. The fat rises rapidly to the surface on standing. If bacterial decomposition has taken place, or if the fat was not neutral in the beginning, some emulsification will result.

Why is the fat emulsified in one case and not in the other? 
3.-Diastatic action.-Prepare a starch paste by boiling $1 \mathrm{~g}$. of powdered starch with 100 c.c. of water. Place in each of two testtubes 15 c.c. of the starch paste.

( 1 ). To one add about 2 c.c. of the pancreatic pulp mixture, mix and place in a water-bath at $40^{\circ}$.

(b). To the second add $1 / 4$ of the boiled pulp mixture and likewise set aside in the water-bath at $40^{\circ}$.

At intervals of about 15 minutes take out a portion, about 2 c.c., from each of the two tubes. Test one-half of each portion with iodine for starch and dextrin. Test the remainder for sugar by boiling with Fehling's solution. How soon does dextrin make its appearance? How early can sugar be recognized? When is the achromic point reached?

Note the change in the appearance of the two tubes and the difference in results. Compare this action of pancreas with that of saliva.

4.- Proteolytic action.-In a 50 c.c. Erlenmeyer flask, provided with a cork, place about $5 \mathrm{~g}$. of fresh fibrin and about $20 \mathrm{c}$.c. of chloroform water. This is prepared by adding 2 c.c. of chloroform to 50 c.c. of water and shaking thoroughly. To the fibrin and chloroform water add about 5 c.c. of the pancreatic pulp and mix well. Render the mixture distinctly alkaline by the addition of a few drops of $\mathrm{Na}_{2} \mathrm{CO}_{3}$ solution $(2 \%)$. Cork the flask and set aside at $40^{\circ}$ for $2-3$ days.

Occasionally examine the contents of the flask and compare the rate of digestion with that of gastric juice. Note that the fibrin does not swell up as in gastric digestion, and that the edges are evenly eaten away.

The contents of the flask are finally slightly acidulated with acetic acid, boiled and filtered. The filtrate may contain albumose, pepton, tyrosin and leucin.

(a). To a portion of the filtrate apply the biuret test in the cold.

(b). To another portion add a few drops of bromine water and shake. A pink to a purple-red color (proteinochrom) develops. This is known as the bromine reaction and is due to an unknown substance, proteinochromogen or tryptophan.

This substance in its composition resembles hæmatoporphyrin and bilirubin, and is also related to the animal pigments as melanin. It would seem as if this substance was utilized to build up hamoglobin and other pigments. 
(c). Concentrate the remainder of the filtrate on a watch-glass to a small volume (a few c.c.) and set aside in a cool place over night. Examine the deposit with the microscope for tyrosin which forms characteristic bundles of needles, and for leucin balls. If no deposit forms concentrate to a thin syrup and again set aside for examination.

\section{Leucin . $\mathrm{C}_{6} \mathrm{H}_{13} \mathrm{NO}_{2}$.}

This compound was formerly considered to be $a$-amidocaproic acid, but the more recent studies of Schulze and Likiernik have shown it to be $a$-amido isobutyl-acetic acid, $\left(\mathrm{CH}_{3}\right)_{2} \mathrm{CH} \cdot \mathrm{CH}_{2} \cdot \mathrm{CH}\left(\mathrm{NH}_{2}\right) \cdot \mathrm{CO}_{2} \mathrm{H}$. Leucin is readily formed and is a constant cleavage product in the decomposition of proteids, gelatin and horn. This decomposition occurs in pancreatic digestion; may be brought about by the action of acids and alkalis at high temperatures; and may also occur as a temporary bacterial product during putrefaction. Plant proteids, as well as animal proteids, can give rise to leucin. It can be readily prepared from proteids, or better white horn, by boiling with dilute $\mathrm{HCl}$. Leucin has been found, in diseased conditions, in various organs and glands of the body, in pus, blood, and in decomposed epidermis such as is found on the feet and between the toes. In the latter case the peculiar odor is largely due to decomposition products of leucin. This compound occurs with tyrosin, in the urine in liver diseases, especially in acute yellow atrophy. They are not present in normal urine.

Leucin is dextro-rotatory in acid or in alkaline solutions, but in neutral solutions is inactive. On heating with baryta it becomes inactive. By the action of Penicillium the inactive form is changed into a lævo-rotatory variety. Several isomeric leucins have been prepared synthetically.

In the pure condition leucin forms glistening white plates, which do not readily moisten when touched with water. As usually met with, however, it forms balls or aggregations of spherical bodies which often show light radial markings and are faintly refractive to light. When 
impure leucin is more readily soluble than when pure. It is readily soluble in water ( 27 parts); more readily in hot water. It is difficulty soluble in alcohol, but is readily soluble in acids and alkalis. It forms salts with acids and bases.

The following experiments are made with leucin furnished by the laboratory:

1.-To a drop of water on a slide add a little leucin, about the size of a pin-head. Observe the behavior on contact with water. Then mix, place on a cover-glass and examine under the microscope. Sketch the crystals observed.

Then add a drop of water to the edge of the cover-glass and gently heat over a flame until the leucin dissolves. Set aside to cool slowly, then examine and sketch the crystals.

2.-To a few c.c. of urine in a watch-glass add a little leucin, mix and heat till it dissolves. Concentrate on the water-bath to a small volume; cover with a beaker and set aside over night. Then place the watch-glass under the microscope and examine with a low power. 'Typical light yellow spherules or mulberry-like masses of leucin will be found. Transfer some of the deposit to a slide and examine with a higher objective.

3.-To about 1 c.c. of water in a test-tube add leucin and shake till it ceases to dissolve. Divide into two portions:

(a). To one add a drop of dilute $\mathrm{HCl}$.

(b). To the other add a drop of dilute $\mathrm{NH}_{4} \mathrm{OH}$.

4.--Place some leucin in a glass tube, about six inches long, and open at both ends, and heat gently in an inclined position. A portion of the leucin is sublimed as a woolly deposit. At the same time an odor of amylamin is given off.

5.-Dissolve some leucin in a little water and render the solution alkaline with $\mathrm{NaOH}$. Then add 1-2 drops of dilute $\mathrm{CuSO}_{4}$ solution. The cupric hydrate precipitate which forms at first, redissolves, since with leucin it forms a soluble compound. The solution is colored blue, and on heating does not reduce. This action of leucin is similar to that of glycocoll, of tartrates, and of bile.

6.-Place in a dry test-tube a piece of solid $\mathrm{KOH}$ about one-half an inch long; add some leucin and a drop of water. Heat cautiously 
till the $\mathrm{KOH}$ melts. Place a strip of moist red litmus paper near the mouth of the tube. What is the result and to what is it due? Pour the melted $\mathrm{KOH}$ into a small beaker, rinse the tube with a little water, and add this to the beaker. Place the beaker in cold water and add very cautiously, drop by drop, $\mathrm{H}_{2} \mathrm{SO}_{4}$ till the solution is acid. Then heat the beaker over a flame and note the peculiar odor of valerianic acid. If the odor is not marked pour the liquid into a test tube, cork and set aside for a day or two. On opening the tube the odor will be perceptible.

7.-Place some crystals of leucin on a platinum foil, add a drop or two of nitric acid ( 1.20 specific gravity) and evaporate carefully to dryness. A colorless scarcely visible residue remains. Now add a few drops of $\mathrm{NaOH}$ and warm. The residue dissolves forming a clear or slightly colored solution. On cautious concentration an oily drop remains which does not moisten the foil but rolls about readily. This is known as Scherer"s test and is rery characteristic.

For the detection of leucin in urine see p. 86.

\section{Tyrosin, $\mathrm{C}_{9} \mathrm{H}_{11} \mathrm{NO}_{3}$.}

Tyrosin has been prepared synthetically and is therefore known to be para-oxyphenyl-a-amidopropionic acid,

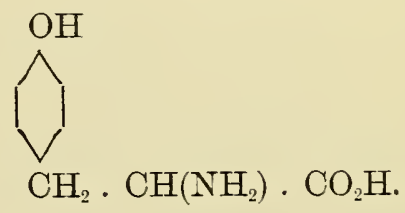

Tyrosin is a constant cleavage product resulting from the action of trypsin, bacteria, acids, or alkalis on animal or vegetable proteids or horn. It is not obtained from gelatin or gelatin-yielding tissues. It is as a rule accompanied by leucin. It is present in old cheese and its name refers to this source. It is present in the intestines during proteid digestion, but is not present in the tissues, blood or urine of the normal body. It is met with in the urine in phosphorous poisoning and in acute yellow atrophy of the liver. 
It forms delicate colorless silky needles which melt at $235^{\circ}$. The crystals often group in bundles and when very impure may form leucin-like balls. It is very difficulty soluble in cold water $(1-2,400)$, more soluble in hot water, insoluble in alcohol or ether. It is readily soluble in dilute alkalis and in dilute mineral acids. In acid solution tyrosin is lævo-rotatory, whereas the synthetic product or that prepared by the aid of alkalis is dextro-rotatory.

The tyrosin necessary for the following experiments is furnished by the laboratory:

1.-Treat a small portion in the same way as in Exp. 1, under Leucin. How can the bundles of fine needles of tyrosin be distinguished from similar bundles of needles of fatty acids? of calcium sulphate?

2.-Test the solubility of tyrosin according to the directions given in Exp. 3, under Leucin.

3.-To some water in a test-tube add a little tyrosin, then a few drops of Millon's reagent. Heat the liquid till it begins to boil. It colors rose-red, and on standing becomes dark-red and may yield a red precipitate. This is known as Hofmann's reaction and is due to the presence of the oxy-phenyl group in tyrosin. What other substances give this reaction with Millon's reagent?

4.-Place some tyrosin in a dry test-tube, add a few drops of concentrated $\mathrm{H}_{2} \mathrm{SO}_{4}$. Place the test-tube in a water-bath and heat at $100^{\circ}$ for about half an hour. Then cool and pour the contents into a small beaker containing some water. To this liquid now add $\mathrm{BaCO}_{3}$ in small portions while stirring, until the reaction ceases to be acid. Filter the liquid and concentrate the filtrate to a very small volume. To this concentrated liquıd add a drop or two of very dilute $\mathrm{FeCl}_{3}$. A beautiful violet color develops. This test is known as Piria's reaction.

5.-Place some crystals of tyrosin on a platinum foil, add nitric acid $(1.2 \mathrm{sp} . \mathrm{g}$.) and warm. The tyrosin becomes bright orange yellow and dissolves. Evaporate very cautiously to dryness when a deep yellow, transparent residue remains. Add a few drops of $\mathrm{NaOH}$ and a deep reddish-yellow solution results. This on evaporation leaves an intense blackish-brown residue (Scherer's test). 
A similar reaction is given by other substances and consequently it is not characteristic.

6.-To a boiling aqueous solution of tyrosin add some one per cent. acetic acid and then sodium nitrite solution, drop by drop. A beautiful red color develops (Wurster).

7.-To a hot aqueous solution of tyrosin add some dry quinone. The liquid becomes colored a ruby-red (Wurster).

\section{* Detection of Leucin and Tyrosin in Urine.}

Tyrosin because of its difficult solubility may occur in the sediment in urine, but if only a small amount is present it may be in solution. Inasmuch as leucin is more soluble it will be, as a rule, in solution in the urine.

Precipitate the urine with basic acetate of lead, filter and remove the lead from the filtrate by hydrogen sulphide. Then concentrate the solution as low as possible and set aside to crystallize. Examine under the microscope for crystals of leucin and tyrosin. If leucin is present it can be removed by means of warm alcohol. 


\section{CHAPTER VII.}

BILE.

Bile is a mixture of the secretion of liver cells and of mucin derived from the cells lining the bile-bladder and duct. It is a thick, tenacious fluid and is alkaline in reaction. The specific gravity ranges from 1.01 to 1.04 . The color of bile varies in different animals. It may be light yellow, brownish yellow, brownish green, green, and greenish blue. Human bile is yellowish, at times greenish. Bile possesses a pronounced bitter taste. It does not coagulate on heating. Human bile contains true mucin, whereas, ox . bile contains but traces of mucin and instead a nucleoalbumin.

The quantity of bile secreted in 24 hours is subject to considerable variation even in health. In the case of fistulæ, from 0.6 to 1 liter of bile has been observed to be secreted in 24 hours, but the secretion under these conditions can hardly be considered as normal bile. The actual quantity given off in a day is probably not less than half a liter. After a proteid diet the secretion is increased, whereas, with fats and carbohydrates it is less marked. The secretion is also decreased in starvation. The secretion is continuous but with variable intensity. Inasmuch as the bile flows from the bladder under very little pressure, a slight obstruction in the duct may lead to retention of the bile. As a result the bile constituents are absorbed and may appear in the urine. Human bile as it is found in the bladder after death has been found to contain from 7-18 per cent. of solids. The bile as it flows from the liver in a fistula, contains much less solids, $1-4$ per cent. 
The bile, therefore, becomes concentrated in the bladder by absorption of water.

Bile contains as characteristic constituents certain salts of bile acids, bile pigments, and small quantities of lecithin, cholesterin, soap, neutral fat, urea, and salts of calcium, magnesium, iron, and copper.

The bile acids are usually present as sodium salts. In some sea-fish they are in combination with potassium. It is customary to speak of two bile acids, glycocholic and taurocholic. The former on cleavage yields glycocoll and cholic acid; the latter taurin and cholic acid. Inasmuch as this cholic acid is but one of several cholic acids known, it follows that there is a group of glycocholic acids and a group of taurocholic acids. Human bile yields three bile acids. The bile of some animals may contain only glycocholic acid, or only taurocholic acid; whereas in some, variable mixtures of the two acids are present. 'Thus, the taurocholic acid predominates in the bile of carnivorous animals, birds, reptiles, and fish. The bile from the rabbit and the hog contains almost entirely glycocholic acid. That from herbivorous animals as a rule contains variable quantities of both. Both glycocoll and taurin are amido acids. Taurin contains $\mathrm{S}$ as a characteristic constituent. According to Hammarsten the bile of some animals contains a third group of bile acids which are rich in $\mathrm{S}$, and which in their behavior to mineral acids resemble ethereal sulphates.

The bile-acid salts are precipitated from their solution in alcohol, on the addition of ether, as fine needles. The bile acids and their salts are dextro-rotatory.

A large number of pile pigments are known, but in normal bile, as a rule, there are but two, bilirubin and biliverdin. The former can be obtained as a reddish yellow, the latter as a greenish powder. The color of bile is 
due to the preponderance of one or the other of these two pigments. Ox-bile has both pigments. The other bile pigments, as bilifuscin, biliprasin and bilicyanin, have been isolated from bile stones and altered bile.

The bile pigments are soluble in alkalis, insoluble in acids, and yield insoluble compounds with calcium and other metals. Bilirubin is slightly soluble in alcohol and in ether, readily soluble in chloroform. Biliverdin is insoluble in chloroform. Bilirubin, in addition to being in the bile, is met with in bile stones as a calcium compound; in old blood extravasations (hæmatoidin); and in urine and tissues during icterus.

The source of bilirubin is undoubtedly hamatin. On reduction it yields hydrobilirubin which is closely related, if not identical, with stercobilin (found in the intestines) and with urobilin of urine. On oxidation it yields biliverdin. The amount of pigment in the bile is usually only a few hundredths of a per cent., rarely 0.1 per cent.

As to the origin of these bile constituents it may be said that the bile acids are elaborated by the cells of the liver, not elsewhere in the body. The bile pigments, without doubt, can be formed in other parts of the body than in the liver, but under normal conditions the liver is the organ where they are formed. Taurin and glycocoll result from the decomposition of proteids in any part of the body.

1.-Place some dilute bile $(1-5)$ in a test-tube and heat to boiling. Immerse a strip of red litmus paper, then remove and wash with water. The reaction is distinctly alkaline.

2.-Place about 5 c.c. of bile in a test-tube, add 10 c.c. of water, mix and filter if necessary. To the clear liquid add acetic acid. A cloudiness or distinct precipitate of mucin or nucleo-albumin forms on standing. This is not marked in ox-bile.

3.-Filter the cloudy liquid obtained in Exp. 2, and apply the biuret test to the clear filtrate. Absence of proteids. Notice also, that the $\mathrm{Cu}(\mathrm{OH})_{2}$ precipitate which forms redissolves in the bile solution and yields a blue liquid which on heating gives a black precipi- 
tate. What is the cause of this black precipitate? What other substances redissolve $\mathrm{Cu}(\mathrm{OH})_{2}$ and yield blue solutions?

4. - To about 20 c.c. of bile in an evaporating dish add about $5 \mathrm{~g}$. of animal charcoal and evaporate on the water-bath, with frequent stirring, to complete dryness. Transfer the residue to a 150 c.c. Erlenmeyer flask, provided with a cork and condensing tube, add about 30 c.c. of absolute alcohol and boil on the water-bath for about half an hour. Cool and filter into a dry flask (or a 50 c.c. test-tube on foot). To the alcoholic filtrate add anhydrous ether till a permanent precipitate forms. Then cork and set aside in a cool place over night. The sodium salts of glycocholic and taurocholic acids crystallize out. Filter off the crystalline deposit and save the filtrate. Squeeze the crystals as dry as possible on the filter in the funnel, then dry between several sheets of filter paper. Save the crystals for subsequent tests.

5.-The alcohol-ether filtrate from the preceding experiment contains, among other things, cholesterin. Place this filtrate in an evaporating dish and allow the ether to evaporate spontaneously, then cautiously evaporate to dryness on the water-bath. Rub up the residue thoroughly, with some ether, filter the ethereal solution into a small beaker or watch-glass, and allow the ether to evaporate spontaneously. Examine the residue under the microscope for the characteristic crystals of cholesterin. Fatty crystals, in the form of needles, are likely to be present.

6.-Detection of bile acids.- On the side table are two sets of bile solutions-bile dissolved in water, and bile dissolved in urine. These solutions are made up in the following strengths: $1-10,1-100,1-500$, $1-1,000$. Apply the following tests first to the "bile-water" dilutions, then to corresponding dilutions of bile with urine. Tabulate the results.

Place about 5 c.c. of each of these solutions in test-tubes and apply the following test, noting carefully the delicacy of the reaction.

(a). To the liquid to be tested add about two-thirds its volume of concentrated sulphuric acid. The acid is allowed to run down the side of the tube slowly, so as not to mix. The temperature should not rise over $60-70^{\circ}$. If necessary, therefore, cool partly under the hydrant, then add 2-3 drops of a solution of cane-sugar (1-10) and tap the tube gently. A pink to a red or violet color develops according to the amount of bile acids present. The foam which forms on shaking is likewise colored pink. This is known as Pettenkofer's test, 
and depends upon the formation of furfurol. An excess of sugar and too much heat must be carefully aroided. Observe the difference in the delicacy of the reactions in aqueous and urine solutions of bile. Oleic acid gires a somewhat similar reaction.

To some water in a test-tube add sulphuric acid as above, then about fire drops of the sugar solution. Notice the yellow to darkbrown color that forms. Repeat this blank test with urine, acid, and fire drops of the sugar solution.

(b). Furfunl test.-Since Pettenkofer's test depends upon the formation of furfurol, out of the sugar added, it can therefore be made with a furfurol solution.

To a few c.c. of the solution to be tested add one drop of a $1.0 \%$ aqueous furfurol solution, then add slowly as in the preceding test about an equal rolume of concentrated sulphuric acid, cool somewhat if necessary, and aroid an excess of furfurol. The reaction is often less intense than in $6 u$.

Apply this test to some diluted bile. Dissolve a little of the crystallized bile acids obtained in Exp. 4 , in some water. Observe the foaming of the liquid when shaken. Divide this solution into two portions and test one according to Pettenkofer: the other with furfurol.

* (c). Detection of bile acids in the urine (Hoppe-Seyler's Method).-The test given above under $6 a$ is usually employed. It should be remembered, however, that substances may be present in urine which will give a reaction similar to that of bile acids. Moreover, in highly colored urines the reaction can be readily masked. In such cases the following method of Hoppe-Seyler, though somewhat long, will give good results. About 100 c.c. of the urine is evaporated to a syrup and the residue extracted with strong alcohol. The alcoholic filtrate is evaporated to dryness, and the residue obtained is dissolved in water. The aqueous solution is precipitated with lead acetate and ammonia. The precipitate is washed, then transferred to an evaporating dish or flask and extracted with boiling alcohol. The alcoholic solution is filtered while hot. A few drops of soda solution are added to the filtrate and this is then evaporated to dryness. 
The dry residue can now be dissolved in water, the solution slightly acidulated with $\mathrm{H}_{2} \mathrm{SO}_{4}$ and filtered. The aqueous filtrate can now be tested directly for bile acids according to $6 a$ or $b$.

7.-Detection of bile pigments. - On the side-table will be found five bottles containing urine diluted with bile in the following proportions: $1-10,1-20,1-50,1-100,1-500$. Apply the following tests to these solutions and tabulate the results.

(a). Gmelin's test.-Place some bile or the suspected urine in a small evaporating dish and add a drop or two of fuming $\mathrm{HNO}_{3}$ - a play of colors, green, blue to violet results. With ox-bile the colors are weak and rapidly change. In urine the green color is especially important since indican may also give a blue color. Various modifications of this test have been suggested and of these the following are especially useful.

(a 1). Filter the bile solution or suspected urine. Then add a drop or two of fuming nitric acid to the moist filter paper. The colored rings are very distinct. This test (Rosenbach's) is much more satisfactory than the preceding and is especially useful when the urine is highly colored.

(a 2). To a few c.c. of fuming $\mathrm{HNO}_{3}$ in a test-tube add slowly some dilute bile solution or the suspected urine, so that the two liquids do not mix. Colors develop at the zone of contact. Finally mix the contents; a decided green color forms, especially on standing.

(b). Huppert's reaction. - To about 10 c.c. of the diluted bile or suspected urine add a little calcium chloride, then an excess of ammonium or sodium carbonate. The bilirubin-calcium compound is precipitated. Filter, wash the precipitate, then transfer while moist to a test-tube and fill it half full of alcohol which has been acidulated with sulphuric acid. Immerse the tube for 10-15 minutes in a waterbath, heated so that the contents of the tube are kept near the boiling point. The solution becomes colored an emerald to a bluish green. Now cool the contents of the tube, then add fuming $\mathrm{HNO}_{3}$. The green color changes to blue, violet, and red. This test is very delicate and is especially useful when the urine is highly colored, or contains much indican or blood pigments.

(c). Iodine test.-Place the diluted bile or suspected urine in a test-tube, incline the tube and add cautiously $2-3$ c.c. of a dilute tincture of iodine so that it forms a layer. Immediately or after a 
few minutes a bright green ring forms at the zone of contact (RosinSmith). This reaction is almost as delicate as that of Huppert. After ingestion of antipyrin the urine will give a similar green ring with iodine.

* (d). Jolles' test. - This is claimed to be the most delicate test for bile pigments. Place 50 c.c. of the suspected urine or a mixture of bile and urine (1-500) in a glass stoppered cylinder, add a few drops of $10 \% \mathrm{HCl}$, then $\mathrm{BaCl}_{2}$ in excess and 5 c.c. of chloroform, and shake vigorously for several minutes. Set aside for about ten minutes for the precipitate and chloroform to settle. Transfer the chloroform and precipitate by means of a pipette to a test-tube. Immerse the tube in a water-bath having a temperature of about $80^{\circ}$. The chloroform evaporates in about 10 minutes. Remove the test-tube and after a few minutes when the precipitate has settled decant the supernatant liquid. The precipitate is colored yellow, if bile pigment is present. Allow three drops of concentrated $\mathrm{HNO}_{3}$ (to which about $1 / 3$ rolume of fuming $\mathrm{HNO}_{3}$ has been added) to run down the side of the tube. The characteristic play of colors develops.

(e). Acidulate some dilute bile with acetic acid, add a few c.c. of chloroform and shake. The chloroform dissolves the bilirubin and is colored yellow. Icteric urine treated in this manner likewise colors the chloroform.

\section{Bile Stones.}

The calculi found at times in the gall-bladder of man consist chiefly of cholesterin. They may be grayish or yellowish white, wax-like in appearance, or may be colored from a light red to a dark brown. The color depends upon the amount of bilirubin present. This pigment is not free but in combination with calcium. The number of stones present in the gall-bladder may vary from a few to several hundred. The size will, therefore, vary considerably, from that of a grain of wheat to stones from $1 / 2-1$ inch in diame- 
ter. As a result of friction the stones frequently show smooth triangular faces. The larger stones when cut in two and polished show generally a concentric arrangement. When they consist of pure cholesterin the stones will float on water. Small amounts of fat may also be present.

The bile-stones as usually found in the gall-bladder of cattle consist largely or wholly of the calcium-bilirubin compound. Similar calculi are met with occasionally in man. These pigment stones may contain metals such as iron and copper, and even at times zinc and manganese. Unlike the cholesterin stones they are always heavier than water.

A third form of bile-stones very rarely found in man consists chiefly of calcium carbonate and phosphate.

Examination of bile-stones.-Pulverize a small bile-stone and place the powder in a test-tube. Add a mixture of alcohol and ether, equal parts, and warm gently until the powder ceases to dissolve. Decant the ether-alcoholic solution into a watch-glass or evaporating dish and allow it to evaporate spontaneously. If the crystals are imperfect redissolve in hot alcohol and allow the solution again to evaporate spontaneously.

Save the crystals for the subsequent tests for cholesterin.

If there is a residue insoluble in the ether-alcoholic mixture add to it some dilute $\mathrm{HCl}$. An effervesence indicates a carbonate $\left(\mathrm{CaCO}_{3}\right)$. If an insoluble residue still remains wash it with water and examine for bile pigments.

Evaporate the $\mathrm{HCl}$ solution to dryness and ignite; then dissolve the residue in dilute $\mathrm{HCl}$ and add $\mathrm{NH}_{4} \mathrm{OH}$. A blue color indicates the presence of copper.

Cholesterin, $\mathrm{C}_{27} \mathrm{H}_{45} \mathrm{OH}$.

This is a common constituent, though in minute quantity, of the normal fluids and tissues of the body. It is very abundant in nervous tissue, especially in the white matter. Under pathological conditions it is met with especially in bile-stones. It is also present in 
atheroma nodules, in tubercular masses, tumors, sputum, pus transsudates and cystic fluids. It is rarely present in the urine and then in small amount. A rare urinary cholesterin calculus has been reported by Horbaczewski. Compounds closely resembling cholesterin, possibly isomers, are found in plants (phytosterins) and by some these have been regarded, though incorrectly, as the source of cholesterin in animals. The cholesterin present in bile passes into the intestines and a small portion may be excreted as such. Most of it, however, is reduced to a hydro-compound -stercorin or coprosterin.

Cholesterin forms white, glistening crystals which under the microscope appear as very thin transparent plates with a more or less notched corner. The crystals melt at $145^{\circ}$ whereas plant cholesterin melts at $133^{\circ}$. It is insoluble in water, in dilute acids, and in alkalis. It is readily soluble in boiling alcohol from which on cooling it recrystallizes. It is readily soluble in ether and chloroform.

1.-Examine under the microscope and sketch the characteristic crystals of cholesterin obtained from a bile-stone.

2.-To some crystals, on a slide under the microscope, add a drop of dilute $\mathrm{H}_{2} \mathrm{SO}_{4}$ (five parts of acid to one part of water). The edges of the crystals show a bright carmine-red color which changes to violet.

3. - To some crystals as in Exp. 2, add a drop of dilute $\mathrm{H}_{2} \mathrm{SO}_{4}$, then a drop of iodine solution. The crystals turn gradually violet, bluishgreen, then blue.

4.-Dissolve a few crystals in a little chloroform in a dry testtube, then add an equal volume of sulphuric acid and shake. The chloroform becomes blood-red, then cherry-red and purple. The acid liquid shows a green fluoresence (Salkowski). The color of the chloroform is quickly discharged if it is poured into a moist test-tube.

5.-Dissolve some cholesterin in 2 c.c. of chloroform, add 10 drops of acetic anhydride, and then drop by drop, concentrated $\mathrm{H}_{2} \mathrm{SO}_{4}$. The mixture becomes red, blue, and finally green (Liebermann's Cholestroul reaction). 
6.-To a little cholesterin in an evaporating dish add a few drops of $\mathrm{HCl}$, and a drop of very dilute $\mathrm{FeCl}_{3}$. On evaporating to dryness a blue color results.

7.-Place a little of the dry cholesterin in a dry test-tube, add 2-3 drops of propionic anhydride and carefully heat over a small flame till melted. On gradually cooling the mass becomes violet, then green, blue and red.

* Detection of cholesterin in urine.-Inasmuch as cholesterin is lighter than water it will be found when present in urine, floating on the surface as a thin pellicle. A microscopic examination will often decide the nature of this film. This is also true of transsudates and other pathological fluids where the crystals are often well formed. Some crystals may be dragged mechanically to the bottom. In the absence of typical crystals it will be necessary to employ the following method:

Extract the urine with ether which takes up fat and cholesterin. Remove the ethereal layer and allow it to evaporate spontaneously. Examine the residue under the microscope for the characteristic crystals of cholesterin. If there is any doubt owing to the presence of fats these must be removed by saponification. For this purpose dissolve the residue in hot alcohol, add some strong alcoholic solution of potassium hydrate and heat on the water-bath for some time. Finally evaporate to dryness, and extract the residue of soaps with ether. This ethereal solution on evaporation will now give a residue free from fat. 
CHAPTER VIII

BLOOD.

Blood is usually a dark-red, thick, opaque fluid. The average specific gravity of normal blood is about 1.058 , and depends primarily upon the amount of hæmoglobin present. It consists of blood corpuscles (red and white) and bloodplates suspended in the liquid portion-the plasma. 'The solid blood corpuscles in man may constitute nearly one-half the weight of the blood. In some animals as the ox, they make up but one-third of the weight of the blood. The blood of adult man contains per cubic millimeter about 5,000,000 red, and 7,500 white blood cells and about 250,000 blood plates. The blood of women contains about $4,500,000$ red cells per cubic millimeter.

The blood possesses a distinct alkaline reaction, due chiefly to sodium carbonate. The alkalinity is decreased considerably in febrile conditions, diabetic coma, cancer, and after excessive muscular exercise. This decrease is due to the increased production of acids, such as sulphuric, phosphoric, and volatile fatty acids, which result from the increased disintegration of protein tissues.

The red blood corpuscles of man and mammals are round, bi-concave discs which contain no nucleus. The llama, camel and related species constitute an exception to the latter statement, since their blood in common with that of birds, amphibians, fish and reptiles, contains nucleated red blood cells which are bi-convex and more or less elliptical. The size of the red blood cell varies somewhat among the different species of mammals, but not sufficiently to enable one to distinguish humau from animal 
blood. This is especially true in the legal examination of blood stains. The presence of blood cells and the production of hæmin crystals justifies the assertion that the stain is due to blood, merely this and nothing more. The size of the cells and absence of nuclei may exclude the blood of certain animals, but it does not prove that it is human blood.

The average diameter of the red blood cells in the blood of man is $7.5 \mu$ or about $\frac{2}{3200}$ of an inch. The blood cells of the ox and horse are about $\frac{1}{2 \frac{1}{2}}$; sheep, about $\frac{1}{50 \pi}$; goat, about $\frac{1}{620}$. The blood corpuscles of but very few mammals are larger than those in human blood; as a rule they are smaller.

The opacity of the blood is due to the suspended blood corpuscles, just as that of milk is due to the suspended fat globules. The cells consist of a stroma or shell which holds in its meshes the hæmoglobin. If the stroma is dissolved or altered so as to allow the hæmoglobin to go into solution the blood then becomes transparent or "laky."

The white blood corpuscles, leucocytes or lymphoid cells, differ considerably in form and size. They are larger and lighter than the red blood cells and contain 1- 4 nuclei. They show amæboid motion. A marked increase in the number of white blood cells (leucocytosis) is observed in leukämia. The leucocytes consist largely of the complex proteid, nucleo-histon. After a rich proteid diet the blood may contain an appreciable amount of albumose, or socalled pepton. This albumose, however, is not in solution in the plasma but is contained within the leucocytes. When these undergo disintegration as in the case of large abscesses, the albumose is set free, and when this is now absorbed by the blood it is promptly excreted by the kidneys and appears in the urine. If albumose (or hæmoglobin) is injected directly into the blood it is excreted at once in the urine. If serum albumin is injected into the blood it is not excreted by the kidneys, but egg-albumin would be excreted. 
The blood plates are supposed by some to be derived from nuclei and hence consist chiefly of nuclein.

The plasma contains about 8.2 per cent. of solids. Of this amount 6.9 per cent. is due to proteins and about 0.87 per cent. due to inorganic constituents, such as chlorides, phosphates, and carbonates.

There are three albuminous substances contained in the plasma, namely: fibrinogen, serum globulin and serum albumin.

Fibrinogen is a most important constituent of plasma. It is, moreover, present in chyle, lymph, exsudates and transsudates. In general it resembles the globulins, but is distinguished from serum globulin especially by its behavior to $\mathrm{NaCl}$, which precipitates it on semi-saturation. Fibrinogen solutions (usually about 0.5 per cent.) coagulate when heated to $56^{\circ}$ or less.

The globulins, fibrinogen and serum globulin, make up most of the proteins of the blood. The relative amounts of globulin and albumin vary in different species and even in the same species of animal. Serum-or para-globulin is unchanged by the clotting of blood. It can be precipitated by a current of $\mathrm{CO}_{2}$, or by saturation with $\mathrm{MgSO}_{4}$. It coagulates at $75^{\circ}$.

Serum albumin is present in plasma, serum, lymph, exsudates and transsudates. Pathologically it may appear, accompanied by globulin, in the urine. It coagulates usually at about $70-75^{\circ}$, but if the solution is concentrated and little or no $\mathrm{NaCl}$ is present it may coagulate at $50^{\circ}$. Serum albumin, and possibly the other proteins of the blood, is made by the epithelial cells of the intestine out of the pepton prepared by the digestive fluids. The pepton that is made in the stomach and in the intestine is not absorbed and carried through the body as such, but is regenerated, synthesized, to serum albumin by the cells of the intestinal wall. The blood coming from the intestines does not contain pepton or albumose in solution. 
Coagulation of blood. - Within a few minutes after blood is taken from an animal it clots, forming a solid jelly. This clot is essentially a net-work of fibrin threads containing in its meshes the blood corpuscles and the fluid part of the blood. Eventually the clot shrinks and a light yellow fluid (blood-serum) is squeezed out. If the blood as soon as it is drawn from the animal is rapidly stirred with the hand, or whipped with a bundle of sticks, glass-rods, or wire, the solid clot will not form, but instead the hand or stirring rods will be covered with shreds of fibrin, or as it is sometimes called "blood fibre." The resulting fluid is spoken of as "defibrinated blood"; it is blood serum containing in suspension blood corpuscles. The fibrin shreds when thoroughly washed, are pure white, and in many respects resemble the coagulated white of an egg.

Coagulation of the blood implies, therefore, the formation of fibrin. This change according to Schmidt is brought about by the action of the fibrin ferment derived from leucocytes, on serum globulin and fibrinogen. Subsequently, Hammarsten showed that the serum globulin was not essential to clotting. That is to say, the fibrin ferment acting on fibrinogen yields fibrin.

When the blood is taken from an animal and received in a flask containing some potassium oxalate it will not clot. The absence of coagulation in this case is due to the precipitation of calcium as an oxalate. In other words, calcium is essential to the formation of fibrin (Arthus and Pagés). The rôle of calcium in coagulation has not been clearly explained until recently.

The fibrin ferment is apparently a globulin, not a nuclein. It is not present in fresh arterial blood (Jakowicki); nor is it present in pepton or histon plasma (Lilienfeld); nor is it present in oxalate plasma (Hammarsten, Pekelharing). The absence of fibrin ferment in the circulating blood has led Lilienfeld to believe that it was not the cause but rather the product of coagulation. This, 
however, is not correct, for while it is true that the fibrin ferment does not exist ready made in the blood the parent substance of the fibrin ferment is present. This zymogen has been designated as mothrombin. It yields the fibrin ferment when calcium is present. Calcium, therefore, is necessary to the formation of the fibrin ferment and not to the fibrin proper. This is seen in the fact that a fibrin ferment, free from calcium, on contact with a fibrinogen solution, likewise free from calcium, yields at once a typical coagulum of fibrin. In order to demonstrate this relation of calcium to fibrin formation it is not necessary to prepare pure fibrin ferment and pure fibrinogen. If ordinary bloodserum is treated with potassium oxalate, to remove calcium, and this serum which contains fibrin ferment is added to oxalate plasma, hence likewise free from calcium, a typical fibrin clot will result.

The present view regarding the clotting of blood may be summarized as follows:

1. - Calcium salts + Prothrombin (zymogen) $=$ Fibrin-ferment.

2. - Fibrin-ferment + Fibrinogen $=$ Fibrin.

Lilienfeld's belief that fibrinogen on contact with nuclein and other compounds yields a cleavage product, thrombosin, which with calcium yields fibrin, is incorrect. In the first place true fibrin contains mere traces of calcium, and secondly, his thrombosin has been shown to be fibrinogen which forms an insoluble calcium compound in slightly alkaline fluids, poor in salts. It is important to note in this connection that calcium is likewise essential to the coagulation of milk. The casein of the millk is changed by rennet to a modified form (paracasein) which unites with calcium to form the curd or cheese (see Exp. 8 under milk). 


\section{Defibrinated Blood.}

\section{Microscopic Examination.}

1.-Examine a drop of fresh blood under the microscope. Measure the diameter and sketch the red and white blood cells. What is the difference between the blood cells of mammals and of birds, reptiles, etc.

2.-Dilute some fresh blood with water and examine as before. Observe and sketch the crenated blood cells.

\section{Spectroscopic Examination.}

1.-Add 1 c.c. of defibrinated blood to 50 c.c. of distilled water and shake thoroughly. Place some of the dilute blood in a test-tube and suspend this about an inch before the slit of the spectroscope (Position No. 1). The test-tube should not be more than one-half inch in diameter. A fish-tail burner placed about three inches from the slit serves as a source of light.

Observe the two absorption bands of oxy-hcemoglotin and their position on the scale in the spectrum.

Place in the flame a platinum wire previously dipped in a solution of sodium chloride. Notice the characteristic yellow line of sodium, its position on the scale, and its relation to the two absorption bands.

2.-Now swing into position the little outside prism of glass so that it shuts off the lower half of the slit. Place a light about three inches in front of the left face of this prism. The spectrum of hæmoglobin appears in the lower half of the field of view while superposed above it is a clear spectrum. Place a tube of the blood, diluted and well shaken as above, between this second light and the left face of the prism (Position No. 2). The spectrum from this tube is now thrown above that from the tube in front of the slit. The two spectra of oxy-hæmoglobin coincide.

(a). To tube No. 1 before the slit, add $1-2$ drops of freshly prepared ammoniacal ferro-tartrate solution (Stokes' solution), and examine at once. The two bands of oxy-hæmoglobin soon disappear, giving place to the single wide band of reduced hremoglobin. Compare this spectrum with the superposed one of oxy-hrmoglobin. Note the change in the color of the blood.

The Stokes' solution is prepared as follows: Dissolve 2 parts of 
ferrous sulphate and 3 parts of tartaric acid in water, then render alkaline by addition of $\mathrm{NH}_{4} \mathrm{OH}$.

(b). To tube No. 2, the one on the left, now add 5-6 drops of strong ammonium sulphide and examine. In a few minutes the single band of reduced hremoglobin takes the place of the two bands of oxyhremoglobin. The spectra of the two tubes now coincide.

3.- To the tube of reduced hrmoglobin in position No. 1, obtained in Exp. $2 a$, add a few drops of concentrated $\mathrm{NaOH}$. The single absorption band becomes replaced by two bands, resembling those of oxy-hæmoglobin, but shifted a little to the right. The left band is the darker of the two. On standing a few minutes the spectrum increases in intensity so that the two bands merge together; in that case dilute with an equal volume of water, and examine again.

This spectrum is due to hemochromogen, or reduced hrematin. This test should be resorted to when the spectrum of hæmuglobin is doubtful.

Compare this spectrum with the superposed spectrum of reduced hæmoglobin $\left(\begin{array}{ll}2 & b\end{array}\right)$. Then substitute for the latter a tube of the diluted, well shaken blood, thus placing the spectrum of oxy-hæmoglobin above that of hæmochromogen.

4.-Dilute some defibrinated blood with about 15 parts of water and shake well. Place some of this solution in a test-tube, in position 1. Superpose the spectrum of oxy-hæmoglobin, using dilute blood $(1-50)$ as in Exp. 2. The upper spectrum of the very dilute blood shows the two bands of oxy-hæmoglobin, whereas the lower spectrum, that of tube No. 1 which contains a strong solution, is entirely dark to the right of the sodium line.

To the tube in front of the slit, position 1, now add 1-2 drops of a fresh, concentrated solution of potassium ferricyanide. The color of the liquid changes to a brown and the spectrum of methomoglobin appears. An intense dark band in the red with two less dark bands to the right. If the liquid is too concentrated dilute with $1 / 4-1 / 3$ its volume of water.

To the solution of methæmoglobin add a few drops of $\left(\mathrm{NH}_{4}\right)_{2} \mathrm{~S}_{2}$. The color and spectrum of oxy-hæmoglobin reappear, and in a short time give way to that of reduced hremoglobin $(26)$.

5.- - To about 10 c.c. of concentrated $\mathrm{H}_{2} \mathrm{SO}_{4}$ in a test-tube add about five drops of blood. Shake thoroughly after the addition of each drop of blood and keep the contents of the tube cool. Note the dark wine-red color of the solution. 
Dilute a portion of this liquid with 2--3 parts of water, cool and examine before the spectroscope (position 1) for the spectrum of hamatoporphyrin. Superpose as in Exp. 2, the spectrum of oxy-hæmoglobin (1-50) for comparison. Hæmatoporphyrin shows a dark narrow band to the left and a wider, darker band to the right of the left band of oxy-hæmoglobin:

Hæmatoporphyrin, $\mathrm{C}_{16} \mathrm{H}_{18} \mathrm{~N}_{2} \mathrm{O}_{3}$, is derived from hæmatin by the splitting off of iron. It results also from the action of $\mathrm{HBr}$ on hæmatin. It is an isomer of bilirubin and has been met with in urine.

6.-To 5 c.c. of diluted blood $(1-15)$ add 2 c.c. of concentrated $\mathrm{NaOH}$. The color changes to a cherry-red. Now heat the tube till the color changes to a brownish-green. Examine before the spectroscope, position 1 , for the spectrum of alkaline homatin. If necessary dilute the contents of the tube $1 / 4-1 / 3$ with water. Alkaline hæmatin shows a dark band through the middle of which passes the sodium line. Superpose the spectrum of oxy-hæmoglobin and compare the two spectra. Then convert the upper spectrum into reduced hæmoglobin as in Exp. $2 a$, and again compare.

7.-Pass a current of illuminating gas for a few minutes through some diluted blood $(1-50)$.

(a). Place a tube containing some of the blood thus treated before the spectroscope in position 1. Superpose the spectrum of oxy-hæmoglobin $(1-50)$. The lower spectrum, due to carbon monoxide hcemoglobin, is nearly the same as that of oxy-hæmoglobin. The two bands, however, are darker and are removed a trifle to the right, so that the two spectra are not exactly continuous. Compare the color of the two tubes.

(b). Now add to each tube $1-2$ drops of Stokes' solution. Carefully note the change in color of the two solutions and also the change in the spectra.

(c). Again superpose the spectrum of oxy-hæmoglobin above that of CO-hæmoglobin. Then add to each tube 5-6 drops of strong $\left(\mathrm{NH}_{4}\right)_{2} \mathrm{~S}_{2}$. Examine at once, and after the lapse of about five minutes.

(d). Again superpose the spectrum of oxy-hæmoglobin above that of CO-hæmoglobin. Then add to each tube one drop of a freshly prepared strong solution of potassium ferricyanide. Examine at once. The oxy-hæmoglobin spectrum changes in a few seconds to 
that of methemoglobin, whereas the spectrum of CO-hæmoglobin persists and is changed only after the lapse of several minutes. Owing to the dilution the spectrum of methæmoglobin will be faint.

CO-hæmoglobin is a much more stable compound than oxy-hæmoglobin and for that reason the color and the spectrum of the solution in experiments $b, c$, and $d$, will change slowly, if at all, whereas oxy-hæmoglobin is readily changed to reduced hæmoglobin in experiments $b$ and $c$, and to methæmoglobin in experiment $d$.

\section{General Reactions.}

1.- Test the reaction of some fresh defibrinated blood.

(a). Dip a moist red litmus paper for a few seconds into the blood, then wash at once in water.

(b). Place a drop of aqueous red litmus solution on a porous porcelain plate. When this has been absorbed apply a drop of blood to the spot and allow this to remain for about a minute. Then wash off with water. Owing to the coloring matter in the blood this method of testing is much more delicate than the preceding.

2.-To some water in a test-tube add a drop or two of blood and mix. Then add tincture of guajac till the liquid becomes cloudy, and finally add some old oil of turpentine. A blue color develops at the zone of contact of the liquids and is due to the oxidation of the guajac. The reaction fails with fresh oil of turpentine owing to the absence of ozone.

(a). This test may be applied to urine, suspected of containing blood, in the following manner: Place in a test-tube equal volumes of guajac and old oil of turpentine. The mixture must not be blue. Now add the urine cautiously so that it forms a layer. If blood is present a bluish-green ring will form at the zone of contact. This is known as Almen's Guajuc Test. The urine, if alkaline, should be neutralized or rendered faintly acid. Pus may give the test with guajac alone.

3.- To 2 c.c. of fresh blood in a test-tube add, without shaking, 2-j c.c. of hydrogen peroxide. Oxygen is liberated abundantly and the liquid foams; the hremoglobin is gradually decomposed. This is due to a so-called catalytic action. 
4. - To some fresh diluted blood $(1-5)$ in a test-tube add ether and gently agitate. The liquid becomes transparent because of the solution of blood cells-laky blood.

5.-Pass a current of illuminating gas for a few minutes through some dilute blood (1-50). Notice the cherry-red color of the solution. As shown above in Exp. 7 (p. 104), CO-hremoglobin is a much more stable compound than oxy-hæmoglobin. The following tests still further serve to demonstrate this fact, and are of great value in distinguishing between the two forms of hæmoglobin. The tests $c$ and $d$ are especially adapted for the detection of small amounts of COhæmoglobin in blood.

(a). In one test-tube place some dilute blood $(1-50)$; in another some of the CO-hæmoglobin solution $(1-50)$. To each of these solutions add half a volume of strong $\mathrm{NaOH}$ solution (1.34 specific grarity). The pure blood solution becomes brownish (due to hæmatin), whereas the CO-hæmoglobin solution is unaltered and retains its cherry-red or pink-red color (Hoppe-Seyler's test).

(b). Place 5 c.c. of the diluted blood $(1-50)$ in a test-tube. In another tube place 5 c.c. of the CO-hæmoglobin solution $(1-50)$. To each tube add an equal volume of fresh, saturated $\mathrm{H}_{2} \mathrm{~S}$-water and shake. The pure blood changes to a green, due to the formation of sulphur-methæmoglobin, whereas the color of the CO-hæmoglobin is unchanged or fades slowly.

(c). In one test-tube place 5 c.c. of the dilute blood $(1-50)$; in another tube 5 c.c. of the CO-hæmoglobin solution. To each of the tubes add 1-2 drops of dilute acetic acid, then one drop of potassium ferrocyanide solution $(1-5)$. The proteids in both solutions are precipitated, but the precipitate in the tube of pure blood is brownish in color, whereas that in the CO-hæmoglobin tube is pink. On standing a while the pink color changes and both precipitates are then alike.

(d). In one test-tube place 5 c.c. of the dilute blood $(1-50)$; in another 5 c.c. of CO-hæmoglobin solution (1-50). To each of these tubes add an equal volume of freshly prepared 1 per cent. solution of tannic acid. The proteids are again precipitated. The precipitate in the tube containing pure blood is colored a grayish brown, whereas that in the CO-hrmoglobin tube is pink. An excess of tannic acid may dissolve the precipitate and should therefore be avoided.

Make a mixture of 1 c.c. of CO-hæmoglobin solution (1-50) and 4 c.c. of oxy-hæmoglobin solution $(1-50)$, add an equal volume of the tannic acid solution and compare with the two tubes obtained above. 
Hamoglobin is readily decomposed on heating with acids or alkalis into globulin and a pigment. If oxy-hxmoglobin is acted upon the pigment that results is hrmertim, whereas with reduced hamoglobin the product is hamochromogen. The latter decomposition has been studied in Exp. 3 (p. 10:3), whereas the formation of hamatin has been observed in Exp. 6 (1. 104), and in Exp. 5 a (p. 106). Hxmatin combines with HCl to form hxmin. When hxmin crystals are dissolved in alkali hamatin is formed.

6. I'remation of homin crystals.-Place in a small Erlenmeyer flask (about 30 c.c. capacity) provided with a cork and condensing tube, 10 c.c. of glacial acetic acid and heat to boiling on the waterbath. Then add, gradually and with constant stirring, 3 c.c. of defibrinated blood. Continue heating on the water-bath for half an hour. Transfer to a small narrow beaker or test-tube and set aside over night. Examine the crystalline deposit microscopically and sketch the form of the hamin crystals.

To preserve the specimen decant the acetic acid; then add 10--20 c.c. of water, stir thoroughly and place aside to settle. Decant off the water and wash in a similar manner with alcohol; then stir up the crystals with ether and transfer to a small filter. Presis the crystals between filter paper till dry, then transfer to a specimen tube. The operation of washing can be greatly simplified by the use of a centrifugal apparatus.

The recognition of hxmin crystals is of the greatest importance in the identification of blood stains. Each student will receive a piece of fabric and a piece of wood stained with blood, or with some red dye. These are examined in the following manner and a report is to be made upon the nature of the stain:

(u). Scrape a little of the stain off the piece of wood. Place the scrapingson a glass slide, add a drop of 1 per cent. solution of $\mathrm{NaCl}$ and warm gently over a very small flame, avoiding ebullition, until the water is nearly driven off. Then, while still moist add $1-2$ drops of glacial acetic acid, cover with a cover-glass and again warm gently over a small flame till most of the acetic acid has evaporated. 
When cool, examine under the microscope for the characteristic light brown hæmin prisms. Sketch the form of the perfect crystals.

(b). Soak the cloth in a 1 per cent. solution of $\mathrm{NaCl}$ in a watchglass and squeeze out the coloring matter as thoroughly as possible. Concentrate the liquid, if it is but weakly colored, on the water-bath to a small volume. Then place $1-2$ drops of the liquid on a glass slide, warm gently, as above under $a$, until the liquid is nearly evaporated, then add 1-2 drops of glacial acetic acid, cover with a glass-slip and again heat till most of the acetic acid has evaporated. Cool and examine for hremin crystals.

7.- The formation of hæmatin and of hæmin crystals may be utilized for the detection of a small amount of blood or blood pigment in the urine. To the suspected urine add $\mathrm{NaOH}$ and boil. The earthy phosphates are precipitated and are colored brownish-red by the hæmatin (Heller's test). If there is doubt as to the nature of the coloring matter in the precipitate, this can be filtered off and subjected to the hæmin test according to the directions given above under $6 a$.

The urine may be precipitated with tannic acid and the precipitate can then be treated for hæmin crystals as above.

8.-Place about 20 c.c. of defibrinated blood in a beaker and add about 200 c.c. of water. Acidulate very slightly with acetic acid, boil and filter. The filtrate should be water-clear. Notice the brown color of the coagulum. To what is it due? Evaporate the filtrate to a small volume, about 20 c.c. If a precipitate forms during the evaporation it should be filtered off. Test the clear, concentrated liquid as follows:

(a). Boil some Fehling's solution in a test-tube, then add some of the liquid and boil again. A yellowish-red precipitate of cuprous oxide indicates the presence of sugar.

(b). Acidulate a little of the liquid with $\mathrm{HNO}_{3}$ and add some $\mathrm{AgNO}_{3}$. A heavy white precipitate soluble in $\mathrm{NH}_{4} \mathrm{OH}$ indicates the presence of $\mathrm{NaCl}$.

(c). Acidulate another portion with $\mathrm{HNO}_{3}$ and add some ammonium molybdate solution. On gentle warming a yellowish precipitate or coloration indicates phosphates. The test for phosphoric acid can be made by adding $\mathrm{NH}_{4} \mathrm{OH}$ to the liquid, then magnesia mixture. A white cloud or precipitate forms if phosphoric acid is present.

(d). Evaporate the remainder of the liquid in a watch-glass on a water-bath till only a few drops remain. Then set aside to cool and examine under the microscope for crystals of $\mathrm{NaCl}$. 


\section{Blood Serum.}

Preparation.-The blood is received directly from an animal into a wide cylindrical vessel or into a common fruit- or battery-jar. It clots in a short time, forming a solid coagulum. The vessel is then placed in an ice-chest for $36-48$ hours. As the clot shrinks the clear yellow serum is squeezed out and collects on top. This yellow serum is removed with a pipette and is used for the follow. ing experiments. It not infrequently happens that the serum as obtained is reddish, due to the presence of blood corpuscles. In that case it is best to place the serum in a tall, narrow beaker and set it aside in the ice-chest for 1-2 days when the corpuscles will subside and leave a straw. yellow, clear serum above.

Blood plasma, the liquid portion of the living blood, contains at least three proteids-fibrinogen, serum albumin, and serum globulin. In the process of clotting the fibrinogen is changed to fibrin and hence the blood serum contains the two proteids serum albumin and serum globulin, or para-globulin.

Carefully review in this connection the work done on the proteids of blood serum (p. 47). What is precipitated if blood serum is saturated with $\mathrm{MgSO}_{4}$ ? With $\left(\mathrm{NH}_{4}\right)_{2} \mathrm{SO}_{4}$ ?

1.-Determine the coagulating point of undiluted blood serum (5-10 c.c.) according to the method given under egg-albumin, Exp. 21, (p. 45). Note the temperature at which the contents of the tube become cloudy: when they gelatinize and when they become solid.

2. - In each of three tubes place 1 c.c. of blood serum. To tube 1 add nothing. To tubes 2 and 3 add 5 and 10 c.c. respectively of distilled water. Immerse in a boiling water-bath for 10 minutes. Note the result. No. 1 coagulates solid, whereas Nos. 2 and 3 do not.

Sufficient dilution of serum with water renders it noncoagrulable by heat. If tap-water is used, owing to the 
presence of calcium salts, partial coagulation will take place.

3.-To each of four tubes add 1 c.c. of blood serum; then add to each 10 c.c. of distilled water. To tubes 1 and 2 add one and five drops respectively of 1 per cent. acetic acid; to tube 3 add a couple of drops of $\mathrm{CaCl}_{2}$ solution; to tube 4 add two g. of $\mathrm{NaCl}$. Immerse the tubes in a boiling water-bath for 10 minutes. Note the results and explain the same.

As shown above in Exp. 2 blood serum diluted with 10 parts of water does not coagulate on heating. In Exp. 3 tube 1 does coagulate, whereas tube 2 does not. To tube 2 now add 1 c.c. of a 10 per cent. $\mathrm{NaCl}$ solution and boil; it coagulates at once. Tube 3 contains a fibrinous coagulum, whereas tube 4 coagulates solid.

What effect would the addition of $\mathrm{NaCl}$ to serum have on the coagulating point?

Compare carefully this and the preceding experiment with Exp. 7 and 8 (p. 41). As shown before even slight excess of acetic acid tends to prevent precipitation of albumin and globulin, whereas $\mathrm{NaCl}$ favors precipitation.

4.-To 5 c.c. of blood serum in a test-tube add 1 drop of formalin, mix and boil. The blood serum does not coagulate.

5. - To 45 c.c. of water in a small beaker add 5 c.c. of blood serum, $\operatorname{mix}$ and filter. Receive the filtrate in a 50 c.c. graduate and place this in a beaker of cold water. Pass a current of $\mathrm{CO}_{2}$ through the diluted serum for about 15 minutes. Then cork and set aside in cold water for some hours. Para-globulin is thrown out of solution as a fine cloud and eventually settles to the bottom as a white precipitate.

6. - To 50 c.c. of water add 1 c.c. of blood serum and mix. To this dilute blood serum apply the following tests:

(a). To about 10 c.c. of the diluted serum add $1-2$ drops of strong $\mathrm{HNO}_{3}$. The cloudiness that forms disappears on shaking. Now heat the contents of the tube to boiling. A yellowish color develops, but no coagulation takes place. Divide the liquid into two portions.

1.- Cool one portion, then add 5-6 drops of $\mathrm{HNO}_{3}$ and boil. Coagulation results.

2.-Raise the other portion to boiling, then add 5-6 drops of $\mathrm{HNO}_{3}$ and boil. Coagulation likewise results. 
(b). To 5 c.c. of the diluted serum $(1-50)$ add an equal volume of water. This gires a serum diluted 1-100. To this very dilute serum add a drop of strong $\mathrm{HNO}_{3}$ and boil. No coagulation. Diride the liquid into two portions.

1.- Cool one portion, then add 5-6 drops of $\mathrm{HNO}_{3}$ and boil. It coagulates.

2.-Raise the other portion to boiling, then add 5-6 drops of $\mathrm{HNO}_{3}$ and boil. The solution remains clear.

Compare these two experiments and explain the difference in results. The precaution that should be taken when testing for small amounts of albumin is emphasized in the next two experiments.

7.- - To 5 c.c. of the diluted serum $(1-50)$ add $5-6$ drops of strong $\mathrm{HNO}_{3}$. A cloudiness forms and on boiling coagulation takes place.

(a). Repeat this experiment with serum diluted as above under $b$ $(1-100)$. Coagulation takes place as in the case of the $(1-50)$ serum.

8.-Boil 5 c.c. of the dilute serum $(1-50)$ and while boiling hot add 5-6 drops of $\mathrm{HNO}_{3}$. Coagulation results. Compare the volume of the precipitate with that obtained in Exp. $6 a$ and 7 .

(a). Repeat this experiment with a serum diluted as above under $b(1-100)$. Only a slight precipitate forms. Compare the rolume of the precipitate with that obtained in Exp. $6 b$ and $i a$. Explain.

It is evident from the above experiments that in the beat and $\mathrm{HNO}_{3}$ test for albumin, in the urine or elsewhere, it is necessary to take into account the amount of $\mathrm{HNO}_{3}$ added and whether the solution is cold or hot. The best result is obtained therefore, when albumin is present in minute quantities, by adding to the cold solution an excess of $\mathrm{HNO}_{3}$ (5-6 drops) to a permanent cloudiness and then boiling. Maximum coagulation will then take place.

$\mathrm{HNO}_{3}$ and heat will coagulate albumin where heat alone will fail to do so. This may be the case if the urine tested has an alkaline reaction. An additional adrantage in the use of $\mathrm{HNO}_{3}$ is that it will dissolve any phospliates that may be thrown out of solution on heating the urine.

9.--To 5 c.c. of the diluted serum $(1-50)$ add 1 drop of 1 per cent 
acetic acid ( 1 c.c. of glacial acid diluted to 100 c.c. with water). A cloudiness results. Test the reaction of the liquid, then boil. A coagulum forms and the liquid is perfectly clear.

(a). Repeat this experiment, first raising the dilute serum to the boiling point and then adding one drop of the 1 per cent. acetic acid. What is the result?

10. - To 5 c.c. of the diluted serum $(1-50)$ add $3-4$ drops of the dilute acetic acid used above. Test the reaction of the liquid, then boil. No coagulation takes place, but the liquid is opalescent.

(a). Boil 5 c.c. of the diluted serum $(1-50)$, and while boiling hot add about 10 drops of 1 per cent. acetic acid and boil again. The cloudiness that forms at first redissolves.

In precipitating proteids, from urine or other solutions, by means of acetic acid and heat, care must therefore be taken to add the acetic acid to the cold solution to neutralization and after that to heat to boiling. Even a slight acidity due to acetic acid will keep albumin in solution.

Compare the behavior of acetic and nitric acids with the serum proteids.

11. - To some of the dilute serum (1-50) add 1-2 drops of $\mathrm{HgCl}_{2}$. A white precipitate forms. Shake up thoroughly and divide into two portions.

( $\iota$ ). To one portion add an equal volume of NaCl solution (1-10). The precipitate promptly dissolves.

(b). To the other portion add an equal volume of undiluted serum. The precipitate likewise promptly dissolves.

The precipitate of mercury and albumin is therefore soluble in $\mathrm{NaCl}$, also in excess of proteids. Of what importance is this fact in practical disinfection? Compare this test with the similar experiment on egg albumin (Exp. 15, p. 44). Note the difference in the behavior of the two proteid solutions.

12.- To some of the dilute serum $(1-50)$ add dilute $\mathrm{CuSO}_{4}$ solution till a precipitate forms. Then add a few drops of strong $\mathrm{NaOH}$ solution (1-3). The precipitate redissolves, yielding a blue solution. 
What other substances give similar solutions of cupric hydrate?

If silver nitrate or lead acetate be added to the dilute serum what would be the result? What is the behavior of the salts of heavy metals with proteids?

The reactions given by serum albumin and serum globulin as worked out in the table (p. 46) will of course be given by the dilute blood serum.

\section{Fibrin.}

The coagulum obtained by whipping freshly drawn blood is cut up into small pieces and washed in running water till perfectly white.

Fibrin on contact with dilute $\mathrm{HCl}$ at $40^{\circ}$ swells up and the contents of the tube become solid in a few minutes (Exp. 1, p. 71). Solution then gradually takes place so that in 2-3 days the fibrin disappears. An acid albumin results (Exp. 4, p. 73).

Fibrin swells up also in 5 per cent. oxalic acid solution, but does not dissolve readily. It is also soluble in dilute neutral salt solutions.

Place in each of two test-tubes about 5 c.c. of hydrogen peroxide. To one add a shred of fresh fibrin. Oxygen is set free especially on slight warming, through so-called catalytic action. This action is probably due to remnants of leucocytes (nucleo-histon).

To the other tube add some boiled fibrin. What is the result?

\section{* Fibrinogen.}

In a two liter flask, or cylinder, place 100 c.c. of a 6 per cent. solution of potassium oxalate. Collect into the flask about two liters of blood, direct from the animal, and mix thoroughly at once. This mixture now contains about 0.3 per cent. of the oxalate which precipitates the calcium salts present and thus prevents coagulation. 
Centrifugate the blood and keep the clear liquid (oxalate plasma) on ice for 24 hours or more. The deposit that forms is largely due to prothrombin, the parent-substance of the fibrin ferment. This is then filtered off. To one liter of clear filtered oxalate plasma add one-half its volume of a previously filtered saturated sodium chloride solution which contains $1 / 2-1$ per cent. of potassium oxalate. Filter off and discard the precipitate.

To the new filtrate add $1-1 \frac{1}{2}$ liters of the filtered saturated $\mathrm{NaCl}$ solution containing potassium oxalate. The liquid now contains for one volume of original plasma two volumes of the salt solution. The fibrinogen separates and floats on the top as a jelly-like mass. Remove this mass with the hand and squeeze as free of liquid as possible. Then place it in a 6 to 8 per cent. solution of salt containing oxalate as above. Most of the precipitate dissolves. Decant carefully the liquid, keeping back the foam and undissolved particles.

This solution of fibrinogen is now purified by a second precipitation by addition of an equal volume of the saturated salt solution containing oxalate. The fibrinogen that is thrown out of solution is removed as before, redissolved and again reprecipitated as above. These three precipitations of the fibrinogen will yield a pure product. The precipitate is finally squeezed, then dissolved in distilled water and filtered. The solution contains pure fibrinogen, free from calcium (Hammarsten).

\section{* Fibrin Ferment.}

Blood serum is saturated with magnesium sulphate. The filtrate is diluted with water and very dilute $\mathrm{NaOH}$ is slowly added, while constantly stirring, till a rather abundant flocculent precipitate of magnesium hydrate forms. This precipitate drags down mechanically the ferment. The precipitate is collected, washed, squeezed as dry as 
possible, then dissolved in water by addition of acetic acid to neutral reaction. The magnesium salts are removed by dialysis and finally the last traces of calcium and magnesium are remored by addition of potassium oxalate $(1 / 2-1$ per cent.). The filtered liquid contains the fibrin ferment, free from calcium, and when added to the fibrinogen solution prepared as above, likewise free from calcium, a precipitate of fibrin promptly forms. 
CHAPTER IX.

MILK.

Milk is a secretion of the mammary gland. It is composed of water, casein, globulin, albumin, fats, milk-sugar, and inorganic salts. The color of milk is due in part to the suspended fat globules, and in part to the casein which is held in solution by calcium phosphate. The specific gravity of milk, from a single animal, may vary considerably; usually from 1.028 to 1.035 , but may be as high as 1.039 . Market milk which is the mixture of the product of several animals always ranges from 1.029 to 1.034 . The average specific gravity of whole milk is placed at 1.029 .

The reaction of milk is usually alkaline or amphoteric. It may however be acid, and this is especially true of carnivorous animals. On standing milk becomes gradually acid owing to the formation of lactic acid by fermentation. Fresh milk does not coagulate on heating. After fermentation sets in milk will coagulate on heating, and later curdles without the application of heat. Sterilized milk, properly kept, will remain sweet indefinitely. The scum which forms on boiled milk is not coagulated albumin but a combination of casein and calcium. When removed a new scum forms on the milk when heated and this may be repeated, again and again. Solutions of casein under similar conditions become covered with scum.

The addition of rennet to milk produces in a short time a solid coagulum, the curd or cheese. The clear liquid remaining is the whey, or milk-serum. The reaction of the 
milk is not affected by this change. The presence of calcium is necessary to the formation of curd. The casein originally present in the milk is apparently changed by the ferment into two proteids. One of these unites with calcium to form the curd and is known as para-casein. The other proteid is formed in small amount, is related to the albumoses, and is known as whey-proteid.

Casein is a complex proteid belonging to the nucleoalbumins. It is insoluble in water but is readily dissolved in the presence of alkalis. A solution in calcium hydrate can be neutralized with phosphoric acid without precipitation of the casein. The milky liquid thus obtained contains, in solution or suspension, the casein and considerable calcium phosphate. Casein is thrown out of solution by dilute acids, or by saturation with $\mathrm{NaCl}$ or $\mathrm{MgSO}_{4}$. It is also precipitated by metallic salts. In the presence of calcium a solution of casein is coagulated by rennet. As in the case of milk, a solution of casein when boiled becomes covered with a scum. On digestion with pepsin it yields pseudo-nuclein which contains phosphorus. The casein in woman's milk is different from that in cow's milk. The former is more difficult to precipitate with acids, salts and rennet. When precipitated by an acid the coagulum is finely flocculent and dissolves readily in an excess of acid, whereas casein from cow's milk is coarsely flocculent and is less readily soluble in excess of acid. Unlike casein from cow's milk it does not yield pseudo-nuclein on digestion. Casein is derived apparently from a nucleo-proteid contained in the protoplasm of the cells of the gland.

The globulin of milk, or lacto-globulin of Sebelien, is probably identical with serum globulin. Lacto-albumin is related to, but not identical with, serum albumin. Like casein and milk-sugar it is a special product of the cells of the gland. Schlossmann found the three proteids present in milk in the following quantities: Casein, 3.19 per cent.; albumin, 0.37 per cent.; globulin, 0.15 per cent. Only traces 
of urea, creatin, etc., are normally present in milk, consequently all the nitrogen present can be considered as contained in the proteid substances.

The fat is present as an emulsion of fat globules. These vary in size in milk from the same species, and from different species. According to Woll they are on an average $3.7 \mu$ in diameter, and from 1 to 5.7 millions of these globules are contained in 1 c.c. of milk. The former belief that the fatty globules were surrounded by an albuminous envelope is no longer held. The fat is supposed to result from a degeneration of the protoplasm of the cells, but it is possible that a part, at least, is brought to the gland by the blood.

The sugar present in the milk, lactose, is a specific product of the gland cells and is not directly derived from the blood. It is possible that it is derived, like casein, from the nucleo-proteids in the cells. That these compounds can give rise to carbohydrates has been demonstrated. In exceptional cases milk-sugar may appear in the urine. Like glucose it is dextro-rotatory and reduces Fehling's solution. Although readily decomposed by bacteria it is not acted upon by pure yeast. This fact as well as its solubility, crystalline form and the formation of mucic acid on oxidation with nitric acid distinguishes lactose from glucose.

The colostrum corpuscles can be considered as epithelial cells'which have taken up fatty globules, rather than as degenerated cells. They are found in milk secreted just before and after delivery. And appear as nucleated, granular cells, containing numerous fatty granules. They are from 5 to $25 \mu$ in diameter. The milk at this time is yellowish in color, alkaline in reaction, and has a high specific gravity 1.046-1.080. When such milk is heated it coagulates solid owing to the presence of increased quantities of albumin and globulin. (See table giving composition of colostrum, Chapter XI). 
1.-Examine a drop of milk under the microscope. Sketch the different sized globules present and measure their diameter. They average about $5 \mu$, but some globules may attain a diameter of $18 \mu$ or more.

2.-Examine microscopically a drop of skimmed milk. What difference is observed between this and whole milk?

3.-Examine with a microscope colostrum milk. Sketch and measure the colostrum corpuscles.

4.-Place about 10 c.c. of milk in a test-tube and boil. Then immerse litmus paper in the hot milk for $1-2$ minutes, remove and examine. Under what conditions does milk become acid?

5.-Boil about 25 c.c. of milk in a small beaker for five minutes. No coagulation proper, but a scum may form. Remove the scum with a spoon or spatula and heat again; a new scum forms. This formation of scum will repeatedly take place. What is the nature of this scum? Casein is not coagulated by heat. Why does not the albumin in the milk coagulate? Save the milk for Exp. 13.

6. - To about 10 c.c. of milk in a test-tube add one drop of dilute acetic acid $(1-10)$, then boil. The casein is coagulated and carries down with it the fat. The serum is clear.

7.- Set aside in a test-tube some milk over night at ordinary room temperature. The next day heat the contents to boiling. Explain the result.

8.-Place 10 c.c. of milk in each of five test-tubes.

To No. 1 add $1 / 2$ c.c. of very dilute $\mathrm{HCl}(10$ drops of $\mathrm{HCl}$ to $50 \mathrm{c.c}$ of water).

To No. 2 add $1 / 2$ c.c. of 2 per cent. $\mathrm{Na}_{2} \mathrm{CO}_{3}$ solution.

To No. 3 add 1/4 c.c. of saturated $\left(\mathrm{NH}_{4}\right)_{2} \mathrm{C}_{2} \mathrm{O}_{4}$ solution $(1-20)$.

Then add to each of these three tubes and also to Nos. 4 and 5 two drops of rennet solution and mix. Heat the contents of tube No. 5 to boiling. Then place all the tubes in a water-bath at $40^{\circ}$ and examine every $3-5$ minutes.

The contents of tube 1 will coagulate in a few minutes; No. 4 next; Nos. 2, 3, and 5 will not coagulate. The latter does not because the heat has destroyed the ferment. The action of renuet is retarded or prevented by alkali, and is favored by acid, such as is present in the gastric juice. 
The coagulum which forms contains para-casein and the fat. The clear liquid that separates from the coagulum on standing is the whey, or milk-serum. Para-casein is different chemically from the casein obtained by the addition of an acid to milk. Calcium salts must be present in order that para-casein may form. Tube No. 3 does not coagulate because the calcium is thrown out of solution as the oxalate. Compare the change that takes place with that in the clotting of blood. If oxalate of sodium is added to freshly drawn blood what is the result?

8 a.-Continue heating tube 3 at $40^{\circ}$ for about $1 / 2$ hour. Then add $2-3$ drops of $\mathrm{CaCl}_{2}$ solution. The liquid instantly solidifies. This shows that the rennet has acted on the casein and changed it into the modification which, with calcium, yields para-casein.

Calcium is likewise necessary to the coagulation of blood, not however, for the formation of clot directly as in the case of the milk curd. Calcium-free blood plasma (oxalate plasma) and calcium-free fibrin ferment when mixed, promptly yield a clot of fibrin. The calcium is necessary to the formation of the fibrin ferment from a parent substance, prothrombin (Hammarsten).

9. -To some milk in a test-tube add $1-2$ volumes of ether, close and shake thoroughly. The fat globules do not dissolve; the milk remains opaque. Now add a few drops of $\mathrm{NaOH}$ and shake again. The ether now dissolves the fat and the liquid clears up. This reaction was taken at one time to indicate that the globules were surrounded by an albuminous envelope. Compare this test with the action of ether on blood.

10.- To some milk in a test-tube add a few drops of $\mathrm{NaOH}$ and heat. The liquid becomes yellow, then orange, and finally brown.

11.-To a 4 per cent. solution of lactose add a little $\mathrm{NaOH}$ and heat. The same color reaction is developed as in Exp. 10, which is due. to the sugar present in the milk.

12.-To some milk add tincture of guajac and mix; then pour on a layer of old turpentine. A deep blue color develops. This test is also given by blood. 
13.--Repeat the preceding experiment using, however, the boiled milk from Exp. 5. The color does not develop. Heat has changed the proteids so that they can no longer assist in the oxidation of the guajac resin.

14. - To about $10 \mathrm{c.c}$. of milk in a test-tube add $5 \mathrm{~g}$. of powdered $\mathrm{MgSO}_{4}$ and shake thoroughly. Then pour onto a filter, resting in a test-tube, and set aside to filter over night. Boil the clear filtratealbumin coagulates. The casein is precipitated almost completely by $\mathrm{MgSO}_{4}$.

15.-To 5 c.c. of milk add 4 volumes ( 20 c.c.) of strong alcohol, shake thoroughly and set aside. All the proteids present are precipitated.

16.-Dilute 10 c.c. of milk with about 30 c.c. of water and divide into three portions.

To 1 add $1-2$ c.c. of potassium alum solution $(1-10)$ and shake. The casein is precipitated and carries down with it the fat.

To 2 add $1-2$ c.c. of copper sulphate solution $(1-10)$ and shake. A voluminous greenish blue precipitate of the proteids present (and fat) results.

To 3 add about 2 c.c. of Almén's tannic acid solution and shake. The proteids are precipitated.

*17. - Moisten a few granules of pepsin with a drop of water, or better with a drop of a 0.7 per cent. $\mathrm{NaCl}$ solution. Then add 5 c.c. of milk, mix and set aside in a waterbath at $40^{\circ}$. Coagulation results in a few minutes. It will fail if more water or salt solution is added to the pepsin (Pekelharing). Chymosin on digestion with pepsin and 0.3 per cent. $\mathrm{HCl}$ is destroyed (Hammarsten).

18.-Add 50 c.c. of milk to about 400 c.c. of water, mix well and while stirring add dilute acetic acid (1-10), drop by drop, till the precipitate becomes coarsely flocculent and ceases to increase. Stir thoroughly and set aside over night. The reaction should be distinctly acid.

The precipitate consists of casein and fat. Filter off the precipitate and allow to drain well, then fold over half the filter in the funnel and apply gentle pressure with the fingers until no more water can be squeezed out. 
Transfer the precipitate to a small dry beaker, add about 30 c.c. of strong alcohol and stir thoroughly so as to dehydrate the casein. Then filter and again squeeze the contents of the filter as dry as possible. Transfer the precipitate to a small dry beaker, add about 50 c.c. of ether and heat on a warm water-bath with constant stirring for about 10 minutes. Owing to danger, the light should be very low or better turned out. Finally transfer the contents to a filter and squeeze as dry as possible.

Spread open the filter on the table, allow the remaining ether to evaporate, then powder. The white chalky powder is casein.

The ether filtrate received in a small beaker or evaporating dish and evaporated cautiously on the water-bath gives the milk-fat.

The aqueous filtrate from the casein and fat precipitate contains albumin and milk-sugar. Place it in a beaker and boil for $\mathbf{1 5}$ minutes. Filter off the precipitate of albumin and reserve for subsequent tests.

Concentrate the filtrate from the albumin, in a beaker on a wire gauze, till it becomes cloudy and bumps. Cool the liquid; the cloudiness disappears and is therefore due to phosphates. Heat again to boiling and filter hot. Concentrate the filtrate now on the waterbath to a syrupy consistency and set aside over night. Crystals of milk-sugar separate on standing.

To the casein obtained in the above, apply the biuret, the Mil lon, and the xanthoproteic reactions. Also dissolve a portion in water to which some $\mathrm{Na}_{2} \mathrm{CO}_{3}$ solution has been added. Observe the cloudiness of the solution. Heat a portion of the casein with alkaline lead acetate. What is the result? Apply the same tests to the specimen of albumin. Review carefully the reactions for lactose and fats. 


\section{CHAPTER X.}

URINE.

To some normal urine apply the following reactions and carefully note the results:

1.-Test the reaction with litmus-paper. What is it?

2. - Heat some urine with a strong acid as $\mathrm{HCl}, \mathrm{HNO}_{3}$, or $\mathrm{H}_{2} \mathrm{SO}_{4}$. Observe the peculiar odor that is emitted and the change in color.

3.- Concentrate some urine in a small dish or porcelain crucible on the water-bath, ignite and test the residue by means of a platinum wire and flame for potassium and sodium.

4.-Add a few drops of oxalic acid or ammonium oxalate solution to some urine. Examine the precipitate under the microscope and test its solubility with acetic and hydrochloric acids. What is it?

5.-To some urine acidified with nitric acid, add a few drops of silver nitrate solution. What is the nature of the precipitate? Test its solubility with ammonium hydrate and with nitric acid. Notice the size of the precipitate.

6.-To some urine acidified with hydrochloric acid, add barium chloride. What does the precipitate indicate? Test its solubility in acids. How large is the deposit?

7.- To some urine add uranium acetate solution. The yellowish white precipitate is uranium phosphate. Test its solubility in mineral acids, and in acetic acid.

8.-Add a few drops of ferric chloride to some urine. What does the precipitate consist of?

9.-Heat some of the urine in a test-tube. If strongly acid no change results, but if it is feebly acid or neutral the phosphate of calcium is precipitated. Why? Write the formula of this salt. To a portion of the urine with this precipitate add nitric acid; cool another portion. What is the result? 
10. - To some urine add ammonium hydrate-ammonium magnesium phosphate and calcium phosphate are thrown down. When the precipitate subsides examine it under the microscope. What is the form of the calcium phosphate? What is the form of the crystals of the ammonium magnesium phosphate? To a portion of the precipitate add acetic acid, to another portion hydrochloric acid. Note the result.

Filter a third portion and to the filtrate add a solution of magnesium sulphate. A precipitate of magnesium ammonium phosphate forms. How does it compare in bulk with that obtained above. Explain the formation of this precipitate. What is meant by earthy phosphates; by alkali phosphates?

11.-Render some urine alkaline with sodium or potassium hydrate. The phosphates of calcium and magnesium are precipitated. Examine the precipitate under the microscope; then test its solubility in acetic and hydrochloric acids. What are the results?

12.-Add a few drops of mercuric nitrate solution to some urine. Observe that the precipitate formed by the first drop redissolves on shaking; that when an excess is added the precipitate is permanent. To a portion of the precipitate add some sodium chloride solution; to another portion add nitric acid. Note the result. The precipitate contains urea.

13. - To about 100 c.c. of urine in a beaker add about 10 c.c. of hydrochloric acid and then set aside for 24 hours. The slight precipitate of reddish crystals consists of uric acid. Examine under the microscope.

14.-Boil some urine with Fehling's solution. What is the result?

15.-Add a few drops of picric acid solution to some urine. Note the presence, or absence of a precipitate. What is it?

16.-To some urine add acetic acid, then a few drops of potassium ferrocyanide. Examine the same as under 15.

17.--Heat some urine with Millon's reagent. A red color would indicate the presence of what substance?

18. - To 5 c.c. of sulphuric acid add about 10 c.c. of urine-a garnet red color is ascribed to "urophæin," a humin substance. 


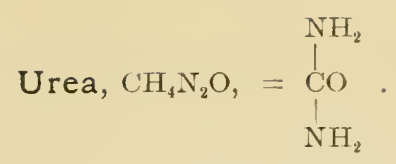

Urea can be considered as an amide of carbonic acid $\mathrm{CO}(\mathrm{OH})_{2}$, and is therefore spoken of as carbamide. It can be prepared artificially by the action of ammonia on carbonyl chloride $\left(\mathrm{CO} . \mathrm{Cl}_{2}\right)$; by heating solutions of ammonium cyanate; by hydration of proteins, creatin, uric acid, etc.

It is the chief form in which waste nitrogen leaves the body. The nitrogen present in the complex proteins, derived from the food and present in the fluids and cells of the body, when disintegration results passes through a series of successive clearage products, each one more simple than the preceding, and eventually appears in the urine as urea or as other waste nitrogenous substances. From 82-88 per cent. of the total nitrogen excreted is eliminated as urea. The remaining $12-18$ per cent. of nitrogen is contained in ammonia, creatinin, uric acid, xanthin bases, indol and a variety of other compounds. It follows therefore that the total amount of urea in a day's urine can be taken as a measure of tissue metabolism, and consequently is of great clinical significance.

While the above is true with reference to man and mammals in general, it does not hold true for birds, reptiles and amphibians, where uric acid may be considered as the chief nitrogenous waste product. Urea is found in minute amount in the blood, lymph, spleen, and liver, but not in muscles. When on account of a disease of the kidneys it cannot be eliminated from the body, it is greatly increased in the blood and elsewhere in the body and may then appear in the sweat, vomit, and in intestinal contents.

The original source of urea is the protein matter of the foods and tissues. In an individual possessing a constant weight, as an adult, the total nitrogen in the food, or an amount corresponding to that, is eliminated by the kidneys 
within 24 hours as waste nitrogen. Some of this waste nitrogen naturally results from the destruction and disintegration of the tissues of the body, and of hæmoglobin. The remainder probably results from the direct breaking down of circulating proteins. It has been supposed that this disintegration of proteins into urea could not be accomplished except through the aid of the living cell. The studies of Drechsel have shown, however, that it is possible to obtain urea by hydration from proteins, whether derived from animals, or from plants.

The immediate antecedents of urea have furnished a fruitful subject for investigation. Apart from the possibility of making urea, by hydration, directly from proteids and from creatin, it may be said that urea results from one of the following antecedents:

Amido acids.

Ammonium carbonate.

Ammonium carbamate.

It is well known that amido acids, as leucin, glycocoll, etc., when fed to animals, increase the urea in the urine. Furthermore, in certain diseases of the liver, amido acids are increased, whereas urea is decreased. When blood containing amido-acids is passed through a freshly removed liver urea is increased. It is certain therefore that these acids may be antecedents of urea. They are probably, however, not changed directly into urea, but first into ammonium carbonate. In general, salts of organic acids are oxidized in the body to the corresponding carbonates. Thus potassium acetate, if administered, appears in the urine as potassium carbonate. Ammonium acetate does not appear in the urine as the carbonate but as urea. Hence amido acids, as amido-acetic acid, are changed first into ammonium carbonate, then into urea.

As stated ammonium carbonate is likewise an antece- 
dent of urea. When this salt is administered urea is increased in the urine. The same is true of the ammonium salts of organic acids since these are oxidized in the body to the carbonate, and the result is therefore the same as if this compound was taken directly. Furthermore, if ammonium carbonate is passed through a perfectly fresh excised liver urea will be formed.

The formation of urea from amido acids, and from am. monium carbonate can further be explained by the formation of ammonium carbamate which may be considered as the immediate antecedent of urea. The relation of these three bodies can be seen from the following formulæ:

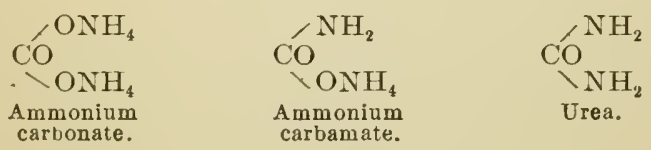

It would appear that the removal of the elements of one molecule of water from ammonium carbonate yields the carbamate, and the removal of a second molecule of water yields urea. Ammonium carbamate is present in the urine of certain animals, and is notably increased after administration of lime water. The lime, it seems, unites with carbamic acid in the body and protects this against conversion into urea. When the blood of the portal vein, instead of passing through the liver, is directed into the vena cava, the liver to all intentional purposes is removed from the body. Consequently the antecedents of urea are not all changed into urea and hence appear in the urine. Carbamic acid appears under these conditions in the urine and the symptoms of intoxication which eventually develop are probably due to carbamates.

The liver is probably the chief, if not the only organ in man where urea is made out of its antecedents. This is seen from the fact that amido acids or ammonium car. 
bonate when passed through an excised liver yield urea. Furthermore, in structural diseases of the liver, as in acute yellow atrophy, urea is diminished considerably and is replaced by its antecedents, ammonia, amido acids, carbamic acid.

The urea which is made in the liver is carried by the blood to the kidneys and there excreted. The epithelial cells lining the convoluted tubules are engaged in the active excretion of urea. If, as the result of inflammatory changes, these cells are altered or destroyed, urea cannot be eliminated from the blood and hence, with other waste products, accumulates in the blood and elsewhere and leads to intoxication-urcemia.

The amount of urea excreted in 24 hours by a healthy adult varies on an average from 25 to 30 grammes. Women excrete somewhat less; children, in proportion to their bodyweight, excrete relatively more than adults. In old age the excretion of urea is diminished.

The most important factor influencing the amount of urea excreted is the kind and quantity of food taken. Thus, with a meat diet a person may excrete $67 \mathrm{~g}$. of urea, whereas with a bread diet the amount may drop to $20 \mathrm{~g}$. It is for this reason that urea is most abundant in the urine of carnivorous animals, and is less abundant in that of herbivorous animals. In starvation, as long as the fats and carbohydrates stored up in the body last the amount of urea is kept low, as in a mixed diet. When these, however, have all been consumed the animal must live on the proteins of its tissues exclusively and urea is at once increased just as if an exclusively meat diet was given. Under these conditions the animal naturally soon perishes.

Urea is not increased by any ordinary amount of muscular exercise. In excessive exercise, pushed to the verge of exhaustion, urea is increased for much the same reasons as in starvation. 
Under pathological conditions urea may be increased considerably. This is especially seen in fevers where, although the amount of food taken is very small, the amount of urea excreted may reach $50 \mathrm{~g}$. per day or even more. The tissue proteins are being actively broken down and hence urea is formed. The fever cannot be considered as the cause of this rapid disintegration but is rather itself a result. Thus, whenever complex substances are broken down, as in the fermentation of sugar, heat is always liberated. In diabetes the urea may be increased; even $100 \mathrm{~g}$. may be daily excreted.

A pathological decrease of urea is of much greater importance. A decrease is met with in but one febrile disease, namely acute yellow atrophy of the liver. In this organ urea is made and hence when diseased the amount of urea in the urine at once falls. For this reason all structural diseases of the liver are accompanied by a decrease in urea. Since the kidney is the organ whereby urea is eliminated it follows that in structural diseases of the kid. neys this elimination will be decreased or even suppressed. In that case urea accumulates in the blood and tissues and is partially excreted by the sweat, vomit and intestinal discharges. Eventually marked intoxication (uræmia) and death result. Poisoning will follow either from non-elimination of urea and other waste products; or from non-formation of urea.

1.- Preparation from the urine.-Concentrate about 500 c.c. of urine on a water-bath to a thin syrup; cool this by immersion in icewater and then add, at the same time stirring well, about three times its volume of strong nitric acid (1.3 specific gravity) which previously has been boiled to expel nitrous acid and then cooled to $0^{\circ}$. Allow the mixture to stand several hours at a low temperature $-0^{\circ}$ is best. Transfer the crystalline mass of urea nitrate which separates out to an asbestos filter (glass-wool or sand), wash several times with small amounts of ice-cold, pure concentrated nitric acid, then dissolve in the smallest possible amount of hot water; cool again and precipitate with concentrated nitric acid. Drain the crystals on a filter as above; 
dissolve in hot water and treat with a small quantity of pure freshly precipitated barium carbonate until effervesence ceases and the solution reacts neutral. Evaporate on a water-bath to dryness, pulverize the residue and extract it repeatedly with cold absolute alcohol whereby the urea is dissolved and the barium salts are left behind. Filter, and, if necessary, decolor the alcoholic filtrate by boiling with animal charcoal, then concentrate to a small volume and set aside for crystals to form.

Write out equations for

1. Urea + nitric acid $=$

2. Urea nitrate + barium carbonate $=$

2.- Synthetic preparation.--Rub up in a mortar $10 \mathrm{~g}$. of thoroughly dehydrated potassium ferrocyanide with $3.75 \mathrm{~g}$. of anhydrous potassium carbonate, transfer to an iron crucible, cover and heat over a Bunsen burner or blast-lamp till perfect fusion results. To the somewhat cooled but still fluid mass add slowly and in small quantities $18.74 \mathrm{~g}$. of well dried red lead, then heat for about ten minutes, at times stirring thoroughly with a glass rod, and finally pour the mass out on an iron plate. Pulverize the cooled mass and dissolve in about 21 c.c. of water; filter the solution of potassium cyanate thus obtained, directly into an evaporating dish containing a solution of $10 \mathrm{~g}$. of ammonium sulphate in about 15 c.c. water. The potassium cyanate is converted into ammonium cyanate. Evaporate the combined aqueous solutions on a water-bath to dryness. As a result of the heating the ammonium cyanate undergoes molecular transposition and becomes converted into its isomer-urea or carbamide. Extract the residue several times with warm absolute alcohol, filter and evaporate the combined alcoholic filtrates on a water-bath almost to dryness, then set aside to crystallize. Purify, if necessary, by re-crystallizing several times from alcohol.

Write out the equations representing the three stages in the above process.

1. $4 \mathrm{~K}_{4} \mathrm{Fe}(\mathrm{CN})_{6}+4 \mathrm{~K}_{2} \mathrm{CO}_{3}+5 \mathrm{~Pb}_{3} \mathrm{O}_{4}=$

2. $2 \mathrm{CNOK}+\left(\mathrm{NH}_{4}\right)_{2} \mathrm{SO}_{4}=$

3. $\mathrm{CN} \cdot \mathrm{ONH}_{4}=$

Why do we add potassium carbonate? Why red lead?

Write equations showing the change that takes place when potassium ferrocyanide is heated by itself; when it is heated with potassium carbonate. 
*Synthetic preparation (Volhard, Ann. 259, 377).-Dissolve $3.9 \mathrm{~g}$. of potassium cyanide and $1 \mathrm{~g}$. of potassium hydrate in 100 c.c. of water, and add slowly a solution of $6.3 \mathrm{~g}$. of potassium permanganate in 100 c.c. of water; keep the temperature below $17^{\circ}$. Now add $10 \mathrm{~g}$. of ammonium sulphate and warm; then filter off the manganese dioxide, evaporate the filtrate to dryness and extract the residue with 95 per cent. alcohol; concentrate the alcoholic solution to crystallization. To remove traces of ammonium chloride dissolve the urea in a little water, add some barium carbonate, evaporate and extract the residue with absolute alcohol.

Why is potassium permanganate used in this method? Write the equation to represent the first stage.

$$
\cdot \mathrm{KCN}+\mathrm{KMnO}_{4}+\mathrm{KOH}+\mathrm{H}_{2} \mathrm{O}=
$$

PROPERTIES OF UREA.

Melting-point.-Determine the melting-point of the urea furnished by the laboratory; of that obtained from the urine and of that prepared synthetically. How do the results compare?

The determination is made as follows: Heat a piece of soft glass tubing in a blast-lamp and when thoroughly softened draw slowly apart. The narrow tube thus obtained is cut up into pieces about five inches long: each of these fused in the middle yields two tubes which are used in making the determination. Fill one of these tubes, sealed at one end, to a height of $1 / 4-1 / 2$ inch with the substance, then fasten this by means of a small rubber band cut from a piece of rubber tubing, to a thermometer so that the sealed end of the tube is on a level with the end of the bulb of the thermometer. Suspend the thermometer with the attached tube in a beaker of about 100 c.c. capacity, containing enough sulphuric acid to clear the substance in the tube as well as the bulb of the thermometer. Now heat with a small flame, stirring constantly, till the substance begins to melt. This is the melting-point; note the temperature.

Taste a specimen of pure urea? What is it like?

Examine the crystals of urea under the microscope; recrysta llize, if necessary. Sketch the form of the crystals observed.

Test the solubility of the urea in water, alcohol and ether. 
Urea nitrate, $\mathrm{CO}\left(\mathrm{NH}_{2}\right)_{2}$. $\mathrm{HNO}_{3}$. - To about $1 / 2$ c.c. of concentrated urea solution or to some urine concentrated to about one-third, add an excess of strong nitric acid free from nitrous acid. If the precipitate which forms does not show distinct crystals, redissolve by aid of heat and pour the contents of the test-tube out into a watchglass. Examine the crystalline form under the microscope and sketch the same. Test the solubility of the crystals in water, alcohol and ether.

Observe the formation of the crystals directly under the microscope, as follows: Place on a slide a drop or so of the urea solution, cover and apply a drop of nitric acid at the edge.

Urea nitrate obtained on decomposition of adenin and bromhypoxanthin does not crystallize in six-sided plates (Krüger).

Urea oxalate, $2 \mathrm{CO}\left(\mathrm{NH}_{2}\right)_{2} \cdot \mathrm{H}_{2} \mathrm{C}_{2} \mathrm{O}_{4}$. - To about $1 / 2$ c.c. of concentrated urea solution add some concentrated oxalic acid solution. Redissolve the precipitate which forms by the application of gentle heat and pour the solution into a watch-glass. Study and sketch the form of the crystals as observed under the microscope. Test the solubility the same as of nitrate.

Observe the formation of the crystals directly under the microscope in the manner given for the nitrate.

\section{MERCURIC NITRATE COMPOUNDS.}

*1. - $2 \mathrm{CO}\left(\mathrm{NH}_{2}\right)_{2} \cdot \mathrm{Hg}\left(\mathrm{NO}_{3}\right)_{2} \cdot \mathrm{HgO}$. - A compound having this composition is obtained when a nitric acid solution of mercuric nitrate is added to a moderately dilute solution of urea nitrate. Examine the crusts which form on standing and sketch the crystals.

*2. - $2 \mathrm{CO}\left(\mathrm{NH}_{2}\right)_{2}, \mathrm{Hg}\left(\mathrm{NO}_{3}\right)_{2} \cdot 2 \mathrm{HgO}$. - This is formed when mercuric nitrate is added to a urea solution as long as a precipitate forms and this then set aside for some time at a temperature of $40-50^{\circ}$. Study and sketch the crystals which result.

3. $-2 \mathrm{CO}\left(\mathrm{NH}_{2}\right)_{2} \cdot \mathrm{Hg}\left(\mathrm{NO}_{3}\right)_{2}$. 3HgO.-A compound possessing this formula is formed when a faintly acid, approximately 7 per cent. 
solution of mercuric nitrate is added to a 2 per cent. solution of urea. Note that the flocculent precipitate which forms becomes granular on standing. One of the methods for the quantitative estimation of urea depends upon the production of this precipitate.

Treat a portion of the precipitate with nitric acid. Note the effect.

Test another portion with salt solution. Observe the result and explain.

Write the equation showing the formation of this precipitate.

Calculate the ratio existing between the molecular weight of urea and that of mercuric oxide from the composition of the above precipitate.

\section{REACTIONS.}

Furfurol test.-Place a few crystals of urea in a porcelain dish, add 1-2 drops of a concentrated aqueous solution of furfurol and 1-2 drops of concentrated hydrochloric acid-a faint yellow color appears which in a few minutes changes to a splendid purple. Intermediate tints of green, blue and riolet may precede (Schiff). This test is also given by allantoin but not by uric acid.

Benzoyl chloride test.-To about $1 / 2$ c.c. of a concentrated aqueous solution of urea, add about 2 c.c. of sodium hydrate solution and $1 / 2$ c.c. of benzoyl chloride, then close the tube with a stopper and shake thoroughly. The tube becomes hot and benzoyl urea, $\mathrm{CO}\left(\mathrm{NH} . \mathrm{COC}_{6} \mathrm{H}_{5}\right)_{2}$, forms. Test a specimen of urine (about 2 per cent. urea) in this way. Note the result and explain. Write the equation representing the reaction between urea and benzoyl chloride $\left(\mathrm{COCl}, \mathrm{C}_{6} \mathrm{H}_{5}\right)$.

This test applied to urine which contains much ammonia will give a precipitate of benzamide.

Bloxam's test.-Acidulate a few crystals with hydrochloric acid and eraporate in a dish to dryness, then heat till white vapors (biuret) cease to be given off. Cool and test according to directions giren under Exp. $d$, p 135.

\section{DECOMPOSITIONS.}

1.-Into ammonia and cyanate.-Urea, as shown, is prepared synthetically from ammonium cyanate and on decomposition it readily yields the compounds from which it is formed. Evaporate to dryness on the water-bath a few c.c. of a solution of urea to which some silver nitrate has been added-silver cyanate and ammonium nitrate form. 
Test a portion of the residue for ammonium by warming with potassium hydrate. Test another portion for silver cyanate as follows: Treat the residue with cold water and filter-silver cyanate is sparingly soluble in water and hence remains on the filter. To prove that this residue consists of silver cyanate, $(1)$ to a portion add ammonium hydrate. What is the result? (2) To another portion add dilute nitric acid-note the effervescence. What is it due to? Write an equation to represent the decomposition of urea in the presence of silver nitrate, also an equation showing the action of nitric acid on silver cyanate.

2.-Into Biuret, $\mathrm{C}_{2} \mathrm{H}_{5} \mathrm{~N}_{3} \mathrm{O}_{2}$. - - Heat some urea in a test-tube till it melts and keep it at that temperature until gas bubbles are freely given off from the fused mass. Test the odor of the gas evolved. What is it? Now set the tube aside to cool, then dissolve in a little water and test as follows for biuret: To the aqueous solution add some potassium hydrate, then a drop or less of a dilute copper sulphate solution. Note the bright pink color (Biuret reaction). What is the result when more copper sulphate is added? The conversion of urea into biuret can best be represented thus:

$$
\begin{aligned}
& \mathrm{CO}<\underset{\mathrm{NH}_{2}}{\mathrm{NH}_{2}} \\
& \mathrm{CO}<\underset{\mathrm{NH}_{2}}{\mathrm{NH}_{2}}=\mathrm{CO}<\underset{\mathrm{NH}_{2}}{\mathrm{NH}}+\mathrm{NH}_{3}
\end{aligned}=\underset{\mathrm{NH}_{3}}{\mathrm{NO}<}
$$

What compounds heretofore studied give a biuret reaction? Biuret unites with metals, as potassium, mercury, copper, nickel and cobalt. With copper and alkali it gives the violet or red color, known as the biuret reaction. If nickel is substituted in this test for copper a yellow or orange color results. The biuret reaction is given by substances containing two amido-carbonyl groups $\left(-\mathrm{CO} . \mathrm{NH}_{2}\right)_{2}$, united to a $\mathrm{C}$ or $\mathrm{N}$ atom, or to a - CO. NH group, or directly to one another, as in oxamide. The proteid molecule contains probably diamides, though not necessarily the biuret group. (Schiff).

3.-Into cyanuric acid, $\mathrm{C}_{3} \mathrm{H}_{3} \mathrm{~N}_{3} \mathrm{O}_{3}$. - Heat some urea, as just given under biuret, till gas ceases to be evolved and the contents solidify to a white chalky mass. Test this for cyanuric acid as follows: 
( $\iota)$. Insoluble in cold, soluble in hot water from which it recrystallizes in prisms.

(b). Dissolve a portion of the residue in cold concentrated sodium hydrate, then heat. The sodium salt which has formed recrystallizes in needles when its solution is heated.

(c). Dissolve a portion of the residue in boiling water and add this to a dilute solution of ammonium cupric sulphate-a beautiful riolet precipitate results. Avoid excess of ammonium hydrate or of copper sulphate.

(d). Dissolve a portion in a few drops of ammonium hydrate; to one-half of the solution add a drop of barium chloride solution-a crystalline precipitate forms; to the remainder add a drop of a weak copper sulphate solution-a violet, crystalline precipitate is produced.<smiles>NC(=O)NC(N)=O</smiles>

4.-Boil for some time an aqueous solution of urea in a test-tube in the mouth of which is placed a moist red litmus paper. What is the result and to what is it due? Write the equation representing the decomposition of urea into ammonia and carbonic acid.

5.-Heat some urea in a test-tube with potassium hydrate. Obserre the odor, and when the tube is cold acidulate with dilute hydrochloric acid-note the effervescence. To what is it due?

6. - To a few c.c. of concentrated urea solution in a test-tube add some nitrous acid, or abaut 2 c.c. of strongly colored nitric acid. What is the result? Write an equation showing the action of nitrous acid on urea, with carbonic acid, nitrogen and water as resulting products.

7.- To some urea solution (or urine) add sodium hypobromite or hypochlorite. Note the effect. The reaction is represented by the equation:

$$
\mathrm{CO}\left(\mathrm{NH}_{2}\right)_{2}+3 \mathrm{NaBrO}=3 \mathrm{NaBr}+\mathrm{CO}_{2}+\mathrm{N}_{2}+2 \mathrm{H}_{2} \mathrm{O} .
$$

8. -To about 2 c.c. of permanganate of potash solution, add some 
concentrated urea solution and then about $1 / 2$ c.c. of concentrated sulphuric acid. Note the effervescence.

$$
2 \mathrm{CO}\left(\mathrm{NH}_{2}\right)_{2}+\mathrm{Mn}_{2} \mathrm{O}_{7}=2 \mathrm{CO}_{2}+\mathrm{N}_{2}+2 \mathrm{NH}_{3}+\mathrm{H}_{2} \mathrm{O}+2 \mathrm{MnO}_{2} \text {. }
$$

Write this equation with the symbol of potassium permanganate instead of $\mathrm{Mn}_{2} \mathrm{O}_{7}$.

9.-Set aside some urine for several days. Then test the reaction; what is it, and to what is it due? What is the cause of this decomposition? To a portion add some dilute acid-observe the effervescence.

Write the equation showing the decomposition of urea into ammonium carbonate.

Allow the litmus paper which was used to test the reaction to dry in the air. Notice that the original color returns. The color was due to volatile alkali-ammonia.

Immerse a piece of red litmus paper in sodium hydrate solution and then set aside to dry. Notice that the change in color remains permanent-due to fixed alkali.

*Detection of urea in liquids other than urine. - The method as given in Exp. 1, p. 129, for the isolation of urea from urine can be employed for the detection of urea in gastric juice, fæces, blood, pus, etc., in pathological conditions. To the material add $3-4$ volumes of alcohol, mix and set aside for 24 hours, then filter and concentrate to a small volume. To this syrupy liquid add $\mathrm{HNO}_{3}$ and examine for crystals of urea nitrate. To the crystals also apply the biuret, furfurol and nitrous acid tests.

\section{Ammonia.}

Normal urine always contains some ammonia, which is not free but combined as a salt,-chloride, sulphate or phosphate. About $0.7 \mathrm{~g}$. of ammonia is excreted daily by an adult. Of the total waste nitrogen from 2-5 per cent. may appear in the urine as ammonia, which therefore may be looked upon as second in importance as a carrier of waste nitrogen. As stated under urea, ammonium acetate or any 
other ammonium salt of an organic acid, when administered, is oxidized in the body to ammonium carbonate which in turn is converted into urea. When the ammonia is united with a strong mineral acid, as chloride, sulphate or phosphate, it passes through the body unchanged and will appear as such in the urine. Because of this union with a strong acid it cannot be converted into urea. Again, if mineral acids are administered, as hydrochloric acid, the amount of the corresponding ammonium salt in the urine will be increased. The acid combines with ammonia and hence this escapes conversion into urea.

The presence of ammonia in the urine is explained by these facts. The sulphur or phosphorus of the food and tissues are oxidized in the body to sulphuric and phosphoric acids respectively. A part of these acids unite with ammonia and the result is the same as if these acids were administered. For this reason the amount of ammonia in the urine after a meat diet is greater than after a vegetable diet; is greater in the urine of carnivorous as compared with herbivorous animals. The urine in starvation will for like reason contain ammonium salts. Some ammonium salts may be introduced with the food, as in the case of cheese, or may be formed as a result of putrefaction in the intestines.

An increase in ammonia may be expected in diseases where the proteins of the body are being unduly disintegrated. This is the case in fever, and in diabetes mellitus. Since urea is made by the liver out of ammonium carbonate or carbamate, it follows that in structural diseases of the liver ammonia in the urine may be greatly increased.

Normal urine, which is acid in reaction when passed, on standing undergoes ammoniacal fermentation due to the introduction of various kinds of bacteria. These organisms may be introduced into the bladder as a result of injury or from the use of unsterilized catheters, and hence this fermentative change may be going on in the urine at the 
time of passage. Such urine will be cloudy, will possess an ammoniacal odor and with acids will effervesce because of the presence of ammonium carbonate. This compound results from the hydration of the urea (Exp. 9, p. 136). The reaction of such urine is alkaline, due to volatile alkali and should be distinguished from that due to fixed alkali, as sodium carbonate.

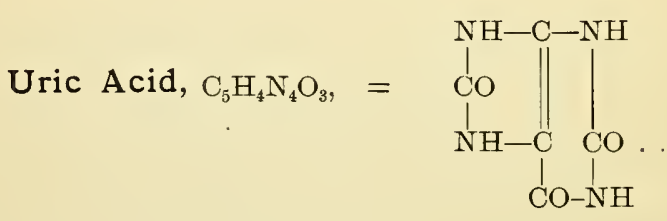

Although uric acid is present in the normal urine in comparatively small quantity, on an average $0.7 \mathrm{~g}$. per day, it nevertheless constitutes one of the most important constituents of urine, especially in disease. As a nitrogen carrier it ranks third, since it contains 1 to 3 per cent. of the total waste nitrogen.

Although uric acid, as can be seen from the structural formula, contains two urea groups and can, as a matter of fact on decomposition, yield two molecules of urea it does not follow that uric acid is an antecedent of urea. When fed to mammals it is broken up in the body and excreted as urea; and it is possible for some of the uric acid made in the body to undergo similar conversion into urea. On the other hand, administration of urea or of ammonium salts to birds is said to increase the amount of uric acid excreted.

It has been shown that urea is the final waste product of proteins. These may be present in the circulating fluids, or in the protoplasm of the cells. The nuclei of cells, however, have a different composition from protoplasm. They contain complex proteids, the so-called nucleo-proteids, which on decomposition yield the nucleins. It has 
been known for some years that nuclein on decomposition with acids or alkalis yields the xanthin bases. As seen from the formulæ xanthin is closely related to uric acid, the latter containing one atom more of oxygen.

Xanthin, $\mathrm{C}_{5} \mathrm{H}_{4} \mathrm{~N}_{4} \mathrm{O}_{2}$.

Uric Acid, $\mathrm{C}_{5} \mathrm{H}_{4} \mathrm{~N}_{4} \mathrm{O}_{3}$.

The source of the xanthin is clearly nuclein, and the relation of these bases to uric acid rendered it probable that the source of uric acid was likewise nuclein. This has been demonstrated by the researches of Horbaczewski. When tissue rich in nuclein is first partially oxidized, then decomposed it yields uric acid. Without oxidation only the xanthin bases would be formed. All tissues containing nucleated cells can be thus made to yield uric acid. The more nucleated cells present, the greater the yield (as from spleen); the fewer nucleated cells present, (tendons, etc.), the smaller will be the yield of uric acid. It is evident, therefore, that uric acid is to be regarded as a specific waste product of nuclein decomposition. Its relation to nucleated cells is seen in leukämia, which is characterized by a large increase in the number of white blood cells. In the urine of this disease xanthin bases and uric acid are greatly increased. Again, certain chemical substances (pilocarpin) which increase leucocytes increase the amount of uric acid, whereas others (quinine, atropine) which decrease leucocytes also decrease the amount of uric acid.

In birds, reptiles, amphibians, etc., uric acid is the chief form in which nitrogen is eliminated. They are different from mammals, in which urea is the chief nitrogenous waste product. The source of the uric acid of birds, etc., is not necessarily the same as that of mammals. This is indicated in the fact mentioned that urea and ammonium salts administered to birds increase the amount of uric acid 
excreted. Moreover, after complete removal of the liver from geese these birds excrete large amounts of ammonia and lactic acid. It is probable, therefore, that in these animals uric acid may be made synthetically in the liver out of ammonia and lactic or other acids. Some uric acid may result, as in mammals, from nuclein decomposition; and since these animals have nucleated blood-cells the amount of uric acid derived from this source may be considerable.

Uric acid, like other nitrogenous constituents of the urine, rises and falls according to the amount of protein matter in the food. This is well illustrated in the following analytical results obtained by Bunge.

\begin{tabular}{|c|c|c|c|}
\hline & Urea. & Creatinin. & Uric Acid. \\
\hline $\begin{array}{l}\text { Meat diet. } \\
\text { Bread diet. }\end{array}$ & $\begin{array}{l}67.2 \mathrm{~g} . \\
20.6 \text { i }\end{array}$ & $\begin{array}{l}2.163 \mathrm{~g} . \\
0.961 \text {. }\end{array}$ & $\begin{array}{l}1.398 \mathrm{~g} . \\
0.253 \mathrm{~g}\end{array}$ \\
\hline
\end{tabular}

Fats and carbohydrates in the food or in the tissues, owing to their proteid saving action, diminish the amount of uric acid excreted as well as that of nitrogenous products in general. Excessive muscular exercise, as in prolonged marches, is followed by an increase in uric acid. In infants and children the uric acid excretion is relatively higher than in adults.

The administration of pilocarpin increases leucocytes and hence increases the amount of uric acid eliminated. Antipyrin, according to some, increases uric acid, whereas others have found a decrease. Conflicting opinions exist as to the effect of sodium salicylate but the preponderance of evidence goes to show that it increases uric acid. Alcohol given to dogs greatly increases the amount of uric acid. It also seems to increase uric acid when given as champagne, but not when given as whisky. Glycerine likewise causes an increased excretion. 
Quinine, in small doses, greatly diminishes uric acid. A decrease is also brought about by atropine and by antifebrin.

While in the case of urea the chief pathological importance is attached to a decrease in the excretion of that body, the reverse is true of uric acid. Uric acid and urates acquire their chief significance when present in excess, and the danger lies in the formation of deposits, eventually of calculi. Some persons exhibit a marked tendency to uric acid excretion and this condition is designated as uric acid diathesis, or lithemia. Stimulants as alcohol, coffee, etc., which experimentally may not induce increased excretion of uric acid in animals are capable of doing this in persons so predisposed.

The most marked and constant increase in uric acid is met with in leukämia. In this disease the white blood-cells are greatly increased. The amount of uric acid may rise to 4 , 5 or even $6 \mathrm{~g}$. per day. Uric acid is furthermore increased in fevers, in pernicious anæmia, arthritis and in certain diseases of the heart and lungs. An excess of uric acid is found in certain nervous disorders as neurasthenia, migraine, epilepsy and chorea, especially after the attacks.

In gout and rheumatism a decrease of uric acid is said to occur, due as is supposed to the retention and deposition of uric acid and urates in the joints. A decrease also exists in diabetes.

Several standards have been employed to decide when uric acid is present in excess. Thus, by some the absolute amount of uric acid excreted in 24 hours is taken as the standard. Since a person normally excretes $0.75 \mathrm{~g}$. per day, $1.5 \mathrm{~g}$. would therefore be considered an excess. On the other hand the amount of urea excreted may be increased at the same time and hence the ratio between urea and uric acid will be the same as in normal urine. Thus $0.75 \mathrm{~g}$. uric acid and $30 \mathrm{~g}$. urea grives a ratio of 1 to $40 ; 1.5 \mathrm{~g}$. of uric acid excreted with $60 \mathrm{~g}$. of urea would still give the normal 
ratio. Consequently the ratio of uric acid to urea ( 1 to 40 ) is frequently taken as the standard of comparison. Another standard is the ratio between the nitrogen contained in the uric acid and the total nitrogen in the urine. Usually the normal quotient of this ratio is placed at 50 .

Uric acid can be readily prepared (1) from the excrement of serpents, (2) from guano, (3) from uric acid calculi, (4) from urine.

* Preparation from guano.-Boil some Peruvian guano repeatedly with milk of lime and water till the solution ceases to become colored. Then extract the insoluble residue with boiling sodium carbonate till the filtrate on addition of hydrochloric acid ceases to give a precipitate. The combined filtrates are treated with sodium acetate and then hydrochloric acid added to a distinct acid reaction. The precipitate which consists of uric acid and guanin, is washed and then boiled with moderately dilute hydrochloric acid whereby the guanin is dissolved while the uric acid remains behind.

Preparation from the urine. 1.-To about 500 c.c. of normal filtered urine, free from albumin, add 10-15 c.c. of concentrated hydrochloric acid and set aside for 24-48 hours. The uric acid deposits in strongly colored crystals. Examine the crystals under the microscope and sketch the several forms observed. The deposit can be purified by dissolving in dilute alkali, then decoloring with animal charcoal and finally reprecipitating the uric acid with hydrochloric acid.

*2.-The best method for the isolation of uric acid, especially when the amount is small, is Ludwig's method, i. e. precipitation with magnesia mixture and ammoniacal silver nitrate. The precipitate is washed with ammonia water then decomposed by warming with potassium sulphide and filtered. The filtrate after addition of hydrochloric acid is concentrated to a small volume, when the uric acid crystallizes. (See Chapter XI). 
* Synthetic preparation. (Horbaczewski). Heat in a test-tube, in a small flame, $0.1-0.3 \mathrm{~g}$. glycocoll with $1-2 \mathrm{~g}$. of urea till the fused mass becomes solid. Avoid heating above $220^{\circ}$. The brownish-yellow mass thus obtained can be tested for uric acid by applying the murexid test (p. 145). To isolate the uric acid the contents of several tubes thus treated are dissolved in boiling water with addition of some ammonium hydrate and the filtrate treated with magnesia mixture and ammoniacal silver nitrate; from the resulting precipitate uric acid is obtained as given under Ludwig's method.

The synthetic process can be represented by the equation:

$$
\mathrm{CH}_{2}\left(\mathrm{NH}_{2}\right) \cdot \mathrm{COOH}+3 \mathrm{CO}\left(\mathrm{NH}_{2}\right)_{2}=\mathrm{C}_{5} \mathrm{H}_{4} \mathrm{~N}_{4} \mathrm{O}_{3}+3 \mathrm{NH}_{3}+2 \mathrm{H}_{2} \mathrm{O} \text {. }
$$

PROPERTIES OF URIC ACID.

Examine under the microscope the form of the crystals of the laboratory specimen and of that obtained form the urine.

Place a few crystals on a slide, add a drop of potassium hydrate solution and watch the solution of the crystals through the microscope. When dissolved apply a drop of concentrated acetic acid to the edge of the cover-glass, and again examine. What is the result?

Test the solubility of the crystals in water, alcohol, ether, potassium hydrate, ammonium hydrate, hydrochloric acid. Uric acid is soluble in sodium phosphate and is reprecipitated by acids (Wulff). About $0.7 \mathrm{~g}$. of uric acid may be held in solution in urine by the urea present. The phosphates may still further increase the solubility. It is very soluble in piperazin, or in lysidin.

\section{SALTS OF URIC ACID.}

Sodium acid urate, $\mathrm{C}_{5} \mathrm{H}_{3} \mathrm{NaN}_{4} \mathrm{O}_{3}$.- - To some uric acid in a testtube add water and boil, then add sodium hydrate, drop by drop, till it dissolves. To the solution thus obtained add sodium bicarbonate, or pass carbonic acid gas till it is almost neutral. Set aside over night to crystallize. Recrystallize, if necessary, from hot water and examine the crytals under the microscope. Sketch a few of the same. What is the formula of the normal sodium urate? 
Potassium acid urate, $\mathrm{C}_{5} \mathrm{H}_{3} \mathrm{KN}_{4} \mathrm{O}_{3}$, is prepared in the same manner as already given, using however, potassium hydrate instead of sodium hydrate. Examine and sketch the crystalline form.

Ammonium acid urate, $\mathrm{C}_{5} \mathrm{H}_{3}\left(\mathrm{NH}_{4}\right) \mathrm{N}_{4} \mathrm{O}_{3}$. -This is readily prepared in the manner already given, by boiling uric acid with ammonium hydrate. If the precipitate fails to dissolve and the solution is strongly ammoniacal, add water till complete solution results, then set aside to crystallize.

Examine the crystals and compare with the preceding.

Does the normal ammonium urate exist?

Calcium urate can be prepared in a similar manner as the preceding by adding calcium hydrate to the boiling mixture till the uric acid dissolves. On cooling the salt crystallizes. Examine as before.

*Sulphate of uric acid, $\mathrm{C}_{5} \mathrm{H}_{4} \mathrm{~N}_{4} \mathrm{O}_{3} .4 \mathrm{H}_{2} \mathrm{SO}_{4}$. Add uric acid to some hot concentrated sulphuric acid as long as it dissolves. On cooling large transparent crystals separate, which on the addition of water decompose into the constituents.

\section{REACTIONS.}

1.-To some uric acid add water, boil, then add ammonium hydrate, drop by drop, till it dissolves. Dilute with an equal volume of water, add hydrochloric acid to acid reaction and immediately after add a solution of phosphotungstic acid-a bright chocolatebrown granular precipitate appears.

2.-To some uric acid dissolved as above, add picric acid-a voluminous yellow precipitate forms (Jaffé).

3. - silver nitrate. Note the result. Now add a little of a solution of a neutral salt as sodium chloride, ammonium sulphate, or better still magnesia mixture-a flocculent or gelatinous precipitate is at once thrown down. It is a compound of uric acid, silver and the base employed. What is magnesia mixture?

4.- To some uric acid add water, boil, and then add a few drops of sodium hydrate and a few drops of Fehling's solution. On heating the white cuprous urate is thrown down. Allow the precipitate to subside, decant the supernatant fluid and add some more Fehling's solution, then boil for some time. The red cuprous oxide is gradually formed. (Compare with test for sugar p. 19). 
This, so-called Drechsel's reuction, has been utilized as the basis of a quantitative method of separation of uric acid and xanthin bases (see alloxuric bodies).

What is Fehling's solution: Write the formula of cuprous oxide.

5.-Dissolve a few crystals of uric acid in a little sodium hydrate, then pour the liquid upon a filter which previously has been moistened with a drop of silver nitrate solution-a yellowish to a brownish-black stain indicates reduced silver. This is a very delicate test and is giren by as little as $\frac{1}{5}$ ro $\mathrm{mg}$. of uric acid.

6.-Murexid test.-Place a minute quantity of uric acid in an eraporating dish, add nitric acid and evaporate to dryness on the water-bath. A yellowish residue results which on contact with the vapors of ammonia turns to a beautiful pink or red (ammonium purpurate or murexid). Now add a drop of potassium hydrate. Note the change in color and the fact that this disappears in a short time on standing-distinction from the xanthin compound, which in addition has more of a red color.

The change that takes place in the above reaction is as follows: The uric acid is oxidized by the nitric acid to alloxantin which is a combination of alloxan and dialuric acid $(\mathrm{CO}<\mathrm{NH}-\mathrm{NH}-\mathrm{CO}>\mathrm{CH}-\mathrm{OH})$. Addition of ammonia converts the latter into dialuramide, (uramil) $\mathrm{CO}<\mathrm{NH}-\mathrm{NH}-\mathrm{CO}>\mathrm{CH}-\mathrm{NH}_{2}$, which with alloxan yields purpuric acid. Excess of ammonia produces ammonium purpurate or murexid (see formula p. 152).

Why is it called murexide? Is purpuric acid known in the free state?

7.-Heat sharply some uric acid in a test-tube. It decomposes into ammonia, hydrocyanic acid (recognized by its peach-blossom odor), urea and cyanuric acid.

DECOMPOSITIONS.

Uric acid on oxidation may yield three distinct groups of products.

1. - In cold acid solution it yields urea and alloxanthe alloxan group. 
2.-In warm acid solution it yields parabanic acid-the parabanic group.

3.--In neutral or alkaline solution it yields allantoin - the allantoin group.

An inspection of the structural formula of uric acid reveals the presence of two urea groups; or we may say one urea and one alloxan group. The first cleavage of the uric acid molecule results in the formation of these two molecules:

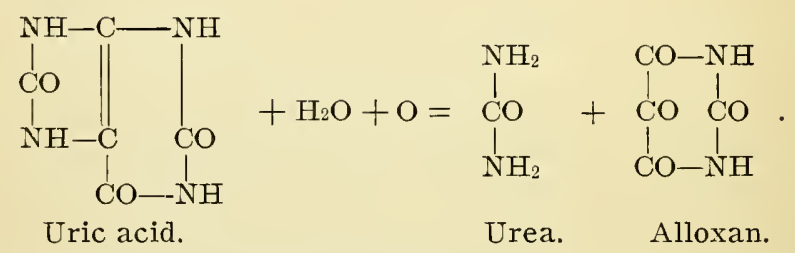

On further oxidation alloxan yields carbonic acid and parabanic acid:

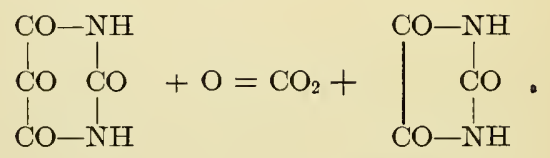

Parabanic acid.

Parabanic acid takes up the elements of water and yields oxaluric acid:

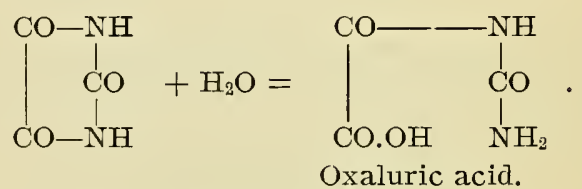

Oxaluric acid on further hydration yields oxalic acid and urea:<smiles>NC(N)=O</smiles> 
On further decomposition the oxalic acid yields $\mathrm{CO}_{2}$ and $\mathrm{H}_{2} \mathrm{O}$; the urea likewise splits up into $\mathrm{CO}_{2}$ and $\mathrm{NH}_{3}$. The uric acid molecule can therefore be easily oxidized to the simple inorganic compounds, $\mathrm{CO}_{2}, \mathrm{NH}_{3}$ and $\mathrm{H}_{2} \mathrm{O}$.

Of the above mentioned decomposition products of uric acid, alloxan (alloxantin) and parabanic acid are not found in the urine. Allantoin and oxaluric acid are present in the urine in small amount.

The fact that oxaluric acid and allantoin, oxidation products of uric acid, are present in urine indicates clearly that a portion of the uric acid formed from nuclein is oxidized in the body, and that possibly only a small amount escapes conversion and appears in the urine.

Oxaluric acid exists in traces as an anmonium salt in the urine. It is not precipitated by calcium chloride and ammonia. On heating with water, acids or alkalis it readily undergoes hydration. It forms a white crystalline powder and yields characteristic ammonium and silver salts.

Allantoin was first met with in the allantoic fluid of the cow. It is found in the urine of infants during the first week after birth. It is present in the urine during pregnancy and is probably present, though in minute quantity, in the urine of adults. It is present in the urine of suckling calves, and at times in the urine of other animals. It has been found in leukämic blood and in ascitic fluids. When uric acid is fed to dogs allantoin is increased. It is also increased after administration of tannic acid, and of diamides.

Allantoin crystallizes in bright, trausparent crystals which are but slightly soluble in cold water. In many of its reactions it resembles urea. The quantitative determination of urea with mercuric nitrate, for this reason, will include allantoin. 


\section{Alloxantin, $\mathrm{C}_{8} \mathrm{H}_{6} \mathrm{~N}_{4} \mathrm{O}_{8}+2 \mathrm{H}_{2} \mathrm{O}$.}

To $5 \mathrm{~g}$. of uric acid in a small Erlenmeyer flask add 10 c.c. of concentrated hydrochloric acid (sp. gr. 1.19) and 10 c.c. of water. Now add very slowly and in small portions $1.5 \mathrm{~g}$. of finely pulverized potassium chlorate. No chlorine or carbonic acid should be evolved. Nearly all the uric acid passes into solution as urea and alloxan, $\mathrm{C}_{4} \mathrm{H}_{2} \mathrm{~N}_{2} \mathrm{O}_{4}$, which on reduction can readily be converted into alloxantin. For this purpose dilute the liquid with an equal volume of water, filter off the unchanged uric acid, and through the filtrate, returned to the flask, pass hydrogen sulphide as long as a precipitate continues to form. Alloxantin is thrown out of solution mixed with free sulphur. Set the flask aside over night in the cold. Filter, and from the precipitate extract the alloxantin by heating it several times with boiling water, and filtering. Alloxantin crystallizes from the filtrate on cooling in colorless prismatic crystals. Filter off the crystals and dry between filter-paper.

*The filtrate from the sulphur-alloxantin precipitate may contain alloxantin. To recover this evaporate in vacuo, extract with alcohol, wash the insoluble portion in cold water and finally crystallize from hot water (Krüger).

Write out the following equations which represent the two stages in the process.

$$
\begin{aligned}
& \mathrm{C}_{5} \mathrm{H}_{4} \mathrm{~N}_{4} \mathrm{O}_{3}+\mathrm{O}+\mathrm{H}_{2} \mathrm{O}= \\
& 2 \mathrm{C}_{4} \mathrm{H}_{2} \mathrm{~N}_{2} \mathrm{O}_{4}+\mathrm{H}_{2} \mathrm{~S}=
\end{aligned}
$$

Alloxantin has been found to be a cleavage product of the convicin of vetch (plant uric acid). It is of interest to note that the latter yields, therefore, a typical murexid test.

1.--Examine the crystals under the microscope and sketch the form. (See Roscoe and Schorlemmer, Vol. III, Part 2).

2.-Place a few crystals in an evaporating dish, and crush; then add a drop of concentrated nitric acid and evaporate gently over a flame, or on a water-bath. Moisten the residue with a little water and add a drop of ammonium hydrate-murexid test.

3.-To a few crystals of alloxantin in a porcelain dish add a drop of ammonium hydrate. Note the results. Then add a drop of potassium hydrate and observe the change, if any. To what is this due? Alloxantin on heating with ammonia yields first uramil, then murexid. 
4.-Dissolre a few crystals of alloxantin in boiling water and add some barium hydrate solution. A violet-blue precipitate forms which on heating becomes white (due to formation of barium alloxantate and dialurate).

5.-To some alloxantin dissolved as above add a little ferrous sulphate, then some ammonium hydrate. A blue color results (Krüger).

6.-To an aqueous solution of alloxantin add some ammoniacal silver nitrate. The silver is reduced. slowly in the cold, rapidly on gentle heating.

$$
\text { Alloxan, } \mathrm{C}_{4} \mathrm{H}_{2} \mathrm{~N}_{2} \mathrm{O}_{4},=\underset{\mathrm{NO}}{\mathrm{CO}-\mathrm{CO}}
$$

* To the powdered alloxantin prepared as just given (about $2 \mathrm{~g}$.) add about 4 c.c. of fuming nitric acid (1.50 sp. gr.) and 2.5 c.c. of concentrated nitric acid (1.t2 sp. gr.). Rub up in a mortar or dish, then set aside in a stoppered testtube for several days till a specimen of the crystals taken out dissolves readily and completely in water, which occurs as soon all the alloxantin has been converted into alloxan. When this is the case transfer the crystals to a porous porcelain plate (or asbestos filter) to dry, then place in a porcelain dish and heat on the water-bath, stirring constantly, till the odor of nitric acid disappears. Recrystallize from a very small amount of hot water.

The reaction is represented by the equation:

$$
\mathrm{C}_{8} \mathrm{H}_{6 \mathrm{~N}} \mathrm{~N}_{4} \mathrm{O}_{3}+\mathrm{HNO}_{3}=2 \mathrm{C}_{4} \mathrm{H}_{2} \mathrm{~N}_{2} \mathrm{O}_{4}+\mathrm{HNO}_{2}+\mathrm{H}_{2} \mathrm{O}
$$

1.-Study and sketch the crystalline form of alloxan. How do the crystals behave on exposure to air?

2.-On boiling with barium hydrate it decomposes into $\mathrm{CO}_{2} \mathrm{H}$

mesoxalic acid, $\mathrm{CO}$, and urea. Write out the equation, $\mathrm{CO}_{2} \mathrm{H}$ 
employing graphic formulæ. On warming with nitric acid it is oxidized to parabanic acid. This, by the action of alkalis, yields oxaluric acid, which in turn decomposes into oxalic acid and urea.

Write out the equations representing these three changes. (See p. 146).

4.-Formation of murexid: Dissolve a few crystals of alloxan in a little water in a dish, evaporate to dryness and add ammonium hydrate.

5.-To an aqueous solution of alloxan add excess of baryta water-a white precipitate of barium alloxantate forms.

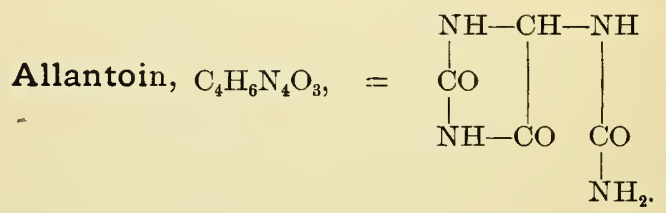

To $4 \mathrm{~g}$. of uric acid, stirred up in about 100 c.c. of water, and warmed, add sodium hydrate till it dissolves, and when cold add gradually $3 \mathrm{~g}$. of powdered potassium permanganate. As soon as all the permanganate has been added and dissolved, filter. Acidulate the filtrate with acetic acid as soon as possible, then set aside in a cold place over night. Filter off the crystals which form and wash with water; combine the wash-water and filtrate, concentrate on the water bath to a small volume, then set aside over night. The crystals that form can be combined with those previously obtained and recrystallized from a small amount of hot water.

The oxidation takes place quantitatirely according to the equation:

$$
\mathrm{C}_{5} \mathrm{H}_{4} \mathrm{~N}_{4} \mathrm{O}_{3}+\mathrm{H}_{2} \mathrm{O}+\mathrm{O}=\mathrm{CO}_{2}+\mathrm{C}_{4} \mathrm{H}_{6} \mathrm{~N}_{4} \mathrm{O}_{3} .
$$

Write out the following equation in which potassium permanganate is used as above.

$$
\mathrm{C}_{5} \mathrm{H}_{4} \mathrm{~N}_{4} \mathrm{O}_{3}+\mathrm{H}_{2} \mathrm{O}+\mathrm{KMnO}_{4}=
$$

1.-Examine and sketch the crystalline form.

2.-Test the solubility in water; also test the reaction. 
3.-To an aqueous allantoin solution add a drop or two of silver nitrate solution. No precipitate forms; then add a drop of dilute ammonium hydrate when a white flocculent precipitate of allantoinsilver, $\mathrm{C}_{4} \mathrm{H}_{5} \mathrm{AgN}_{4} \mathrm{O}_{3}$, results. Test the solubility in ammonium hydrate and in nitric acid; also examine the precipitate under the microscope -it appears as droplets.

4.-To some allantoin solution add mercuric nitrate when a white flocculent precipitate forms. Compare with the behavior of urea (p. 132). To the precipitate add ammonium hydrate and warm. Note the change and explain.

5.-Allantoin, like urea, gives the furfurol test (p. 133), except that it comes slower and is less intense.

6.-It does not yield the murexid test.

7.-On prolonged boiling with Fehling's solution it yields cuprous oxide. Compare with uric acid (p. 144).

8.-Dissolve some allantoin in a little potassium hydrate and divide the solution into two portions. To one add acetic acid-the allantoin is precipitated. Set the other portion aside for several days, then add acetic acid-no precipitate forms. The allantoin has been converted into allantoic acid, $\mathrm{C}_{4} \mathrm{H}_{8} \mathrm{~N}_{4} \mathrm{O}_{4}$.

9.-On heating with acids, allantoin yields allanturic acid, $\mathrm{C}_{3} \mathrm{H}_{4} \mathrm{~N}_{2} \mathrm{O}_{3}$, and urea,

$$
\mathrm{C}_{4} \mathrm{H}_{6} \mathrm{~N}_{4} \mathrm{O}_{3}+\mathrm{H}_{2} \mathrm{O}=\mathrm{C}_{3} \mathrm{H}_{4} \mathrm{~N}_{2} \mathrm{O}_{3}+\mathrm{CO}\left(\mathrm{NH}_{2}\right)_{2} \text {. }
$$

10.-Boil some allantoin with concentrated sodium hydrate till ammonia vapors are given off (what does this indicate?); then acidulate with acetic acid, and add a few drops of calcium chloride solution. Test the precipitate with acetic and hydrochloric acids. What is it? Alkalis yield the same products as acids, but on prolonged heating the allanturic acid decomposes into hydantoic and parabanic acids. The parabanic acid in turn is decomposed into oxalic acid and urea.

$$
\begin{aligned}
& 2 \mathrm{C}_{3} \mathrm{H}_{4} \mathrm{~N}_{2} \mathrm{O}_{3}=\underset{\substack{\text { Hydantoinic } \\
\text { acid. }}}{\mathrm{C}_{3} \mathrm{H}_{6} \mathrm{~N}_{2} \mathrm{O}_{3}}+\underset{\text { Parabanic acid. }}{\mathrm{C}_{8} \mathrm{H}_{2} \mathrm{~N}_{2} \mathrm{O}_{3} .} \\
& \mathrm{C}_{3} \mathrm{H}_{2} \mathrm{~N}_{2} \mathrm{O}_{3}+2 \mathrm{H}_{2} \mathrm{O}=\mathrm{H}_{2} \mathrm{C}_{2} \mathrm{O}_{4}+\mathrm{CO}\left(\mathrm{NH}_{2}\right)_{2} .
\end{aligned}
$$


FORMULA OF URIC ACID AND ITS CHIEF DERIVATIVES.

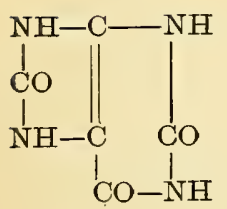

URIC ACID.

$\mathrm{C}_{3} \mathrm{~N}_{4} \mathrm{~N}_{4} \mathrm{O}_{3}$.<smiles>O=C1NCC2NC(=O)N1O2</smiles>

ALLOXAN.

(Mesoxalyl Urea).

$\mathrm{C}_{4} \mathrm{H}_{2} \mathrm{~N}_{2} \mathrm{O}_{4}$.

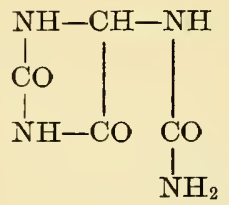

ALLANTOIN. (Glyoxyl-diureïd).

$\mathrm{C}_{4} \mathrm{H}_{6} \mathrm{~N}_{4} \mathrm{O}_{3}$.<smiles>NC(=O)C(=O)NC(=O)O</smiles>

ALLOXANIC ACID.

$\mathrm{C}_{4} \mathrm{H}_{4} \mathrm{~N}_{2} \mathrm{O}_{5}$.

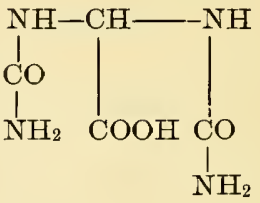

ALLANTOIC ACID.

$\mathrm{C}_{4} \mathrm{H}_{8} \mathrm{~N}_{4} \mathrm{O}_{4}$.<smiles>O=C1CC(O)C(=O)N1</smiles>

DIALURIC ACID. (Tartronyl Urea).

$\mathrm{C}_{4} \mathrm{H}_{4} \mathrm{~N}_{2} \mathrm{O}_{4}$.<smiles>O=C1NC(=O)C(O)N1</smiles>

ALLANTURIC ACID (Glyoxyl Urea).

$\mathrm{C}_{3} \mathrm{H}_{1} \mathrm{~N}_{2} \mathrm{O}_{3}$.<smiles>O=C1CC(=O)NC=N1</smiles>

BARBITURIC ACID. (Malonyl Urea).

$\mathrm{C}_{4} \mathrm{H}_{4} \mathrm{~N}_{2} \mathrm{O}_{3}$.

Alloxantin (Alloxan + Dialuric Acid), $\mathrm{C}_{8} \mathrm{H}_{4} \mathrm{~N}_{4} \mathrm{O}_{7}$.

Purpuric Acid (Alloxan + Dialuramid), $\mathrm{C}_{8} \mathrm{H}_{5} \mathrm{~N}_{5} \mathrm{O}_{6}$.

Ammonium purpurate or murexid (Alloxan + Ammonium dialuramid), $\mathrm{C}_{8} \mathrm{H}_{4} \mathrm{~N}_{5} \mathrm{O}_{6}\left(\mathrm{NH}_{4}\right)$.<smiles></smiles>

PARABANIC ACID. (Oxalyl Urea).<smiles>NC(=O)C(=O)O</smiles>

OXALURIC ACID.

$\mathrm{C}_{3} \mathrm{H}_{2} \mathrm{~N}_{2} \mathrm{O}_{3}$.

$\mathrm{C}_{3} \mathrm{H}_{4} \mathrm{~N}_{2} \mathrm{O}_{4}$.

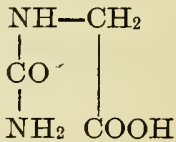

BYDANTOIC ACID.<smiles>NC1C(=O)N=CNC1=O</smiles>

MIDO-BARBITURIC ACID. (Uramil. Dialuramid).

$\mathrm{C}_{4} \mathrm{H}_{5} \mathrm{~N}_{3} \mathrm{O}_{3}$. 


\section{Xanthin or Nuclein Bases.}

As has been mentioned under uric acid (p. 139), these bases are formed from nuclein on simple cleavage (see also nucleo-histon, and alloxuric bases). They have therefore the same source as uric acid. Most of these bases have been found in urine, though in small amount. Inasmuch as they are basic substances made by the animal cell they belong to the group of compounds known as leucomains. The following table will serve to indicate the close relationship that exists between these several bases:

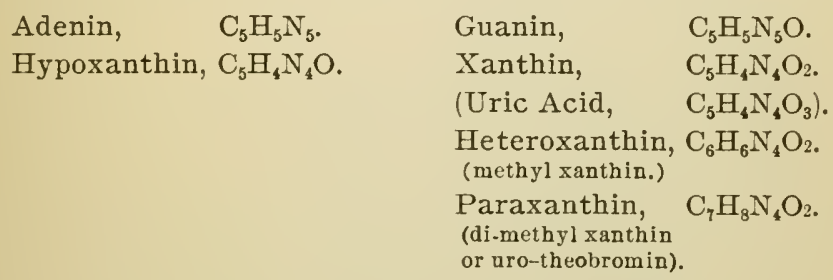

It is interesting to note that theobromin, the active principle of theobroma cacao, is also a di-methyl xanthin and is isomeric with paraxanthin of the urine. Furthermore, caffein or thein, the active principle of coffee and tea, is a tri-methyl xanthin and can be made artificially from xanthin-a waste product of the animal cell. Adenin can readily be converted into hypoxanthin; and guanin can be changed by the same process into xanthin. The conversion of xanthin into uric acid has long baffled the chemist, but this has at last been accomplished as seen by the researches of Fischer. All the members of this group may therefore be prepared synthetically.

$$
\text { Creatinin, } \mathrm{C}_{4} \mathrm{H}_{7} \mathrm{~N}_{3} \mathrm{O},=\mathrm{NH}=\mathrm{C}<\underset{\mathrm{N}\left(\mathrm{CH}_{3}\right)-\mathrm{CH}_{2} \text {. }}{\mathrm{NH}}
$$

Creatinin is derived from creatin, which can be considered as a cleavage product of protein matter. Although 
creatin is abundant in muscles ( 0.3 per cent.) it is not present in the urine, but is represented by its anhydride creatinin. This dehydration probably takes place in the kidney. In alkaline urine the creatinin may be changed back into creatin.

The chief source of creatinin in the urine is not the creatin in the muscles of the body but rather the creatin present in the food. The more meat eaten, the greater is the amount of creatinin in the urine. Milk contains no creatin and hence when this is used as the only food, no creatinin appears in the urine. The mass of the creatinin present in the urine cannot therefore be considered as a waste product of the tissues.

A small portion of the creatinin may be derived from the creatin existing in the muscles. This portion can therefore be considered as a waste product of the body. The mass of the creatin existing in the muscles of the living body undoubtedly undergoes disintegration and is eliminated, probably as urea. In starvation, however, this is not true since creatinin continues to be excreted.

An adult man excretes daily about $1.0 \mathrm{~g}$. of creatinin; women excrete about one-third less. The actual amount of nitrogen that thus leaves the body is as great if not greater than that contained in uric acid.

Creatinin is increased in the urine in diabetes, due to increased meat diet. In general, the excretion of creatinin is parallel with that of urea and of uric acid.

In starvation the amount of creatinin is diminished somewhat but it may remain close to the normal. As the muscle tissue is consumed the creatin is liberated and passes through the body the same as if it were given with meat food. For this same reason there is an increase in creatinin in the urine in febrile diseases. In convales. cence it is decreased. After ordinary muscular exercise creatinin is not increased in the urine; but if the exercise is carried to exhaustion an increase may result. In such 
urine a leucomaïn, xantho-creatinin, $\mathrm{C}_{5} \mathrm{H}_{10} \mathrm{~N}_{4} \mathrm{O}$, has been found. It is said to be decreased in advanced Bright's disease and this retention of creatin is regarded by some as one of the causes of the uræmic symptoms.

* The following method of isolation can also be used for quantitative estimation (Neubauer's method): 200-300 c.c. of urine are rendered alkaline with calcium hydrate and then calcium chloride is added to completely precipitate the phosphates. Baryta mixture can be used instead of calcium chloride. The precipitate is filtered off and washed; the filtrate and wash-water are combined, slightly acidulated with acetic acid, and evaporated to a syrup. This while warm is mixed with 50 c.c. of $95-97$ per cent. alcohol and the mixture transferred to a beaker, covered and allowed to stand eight hours in the cold. The precipitate is then filtered off, washed with alcohol, and the filtrate and washings are concentrated to 50-60 c.c. When cold one-half of a c.c. of a zinc chloride solution, of a specific gravity of 1.20 and free from acid, is added. The thoroughly stirred mixture is covered and allowed to stand in a cold place for two or three days. The precipitate is collected upon a small weighed filter. The filtrate can be used to transfer all the crystals. These are then washed with a little alcohol, till all the chlorides are removed, and finally dried at $100^{\circ}$ and weighed. 100 parts of creatinin zinc chloride contains 62.42 parts of creatinin. From this compound the pure creatinin can be obtained by heating with lead hydrate. The solution is filtered, decolored with animal charcoal, evaporated to dryness and the residue extracted with strong alcohol (creatin remains undissolved). The alcohol solution can be concentrated to crystallization and, if need be, the crystals can be further purified by recrystallization from water. 
Hippuric Acid, $\mathrm{C}_{9} \mathrm{H}_{9} \mathrm{NO}_{3},=\mathrm{C}_{6} \mathrm{H}_{5} \mathrm{CO} \cdot \mathrm{NH} \cdot \mathrm{CH}_{2} \cdot \mathrm{CO}_{2} \mathrm{H}$.

Hippuric acid has been designated as a facultative waste product. That is to say, it is not a direct product of tissue change but is made in the body whenever benzoic acid is present. Hippuric acid results from the union of benzoic acid and glycocoll. Glycocoll, or amido-acetic acid, is an intermediate nitrogenous waste-product which, as an amido acid, eventually yields urea and hence does not appear in the urine. If, however, some substance is present which will unite with it and thus protect it against oxidation it then is excreted in this complex form. Benzoic acid possesses this affinity for glycocoll and hence if it is present in the food, or is formed by protein decomposition in the intestine, or is directly administered, it unites with glycocoll and is excreted as hippuric acid. A similar conjugation is seen in the glycocholic acid of bile. The glycocoll in this case unites with cholic acid. The resultant bile acid is excreted into the intestine where it is decomposed into its components. The glycocoll thus set free may be reabsorbed and if benzoic acid is present may now yield hippuric acid. The source of the glycocoll present in hippuric acid then is tissue metabolism and it is obtained either directly, or after it has passed out with the bile as glycocholic acid.

The benzoic acid which is necessary to hippuric acid formation is derived first from benzoic acid and its derivatives that may exist preformed in the food. These compounds are present in plant foods, especially fruits such as plums, prunes, huckleberries, mulberries, cranberries, etc. The protein molecule contains, as is indicated by the color reactions, an aromatic nucleus. When therefore the protein molecule is split up by bacteria various aromatic bodies result. Phenyl-propionic acid is thus formed and in the body becomes oxidized to benzoic acid. Consequently a second source of benzoic acid is the putre- 
faction of proteins in the intestines. Plant food is not wholly necessary in order that hippuric acid shall form, since with an exclusively meat diet, or in starvation, this acid continues to be elaborated. It is possible that as a third source very small amounts of benzoic acid are formed by tissue metabolism. The fact that in starvation hippuric acid is still eliminated does not prove that benzoic acid is made by the tissues, since even then bacterial growth in the intestines is not wholly suppressed.

The interesting studies of Nuttall and Thierfelder on "sterile" guinea-pigs show that even in the absence of bacteria from the intestines aromatic oxy-acids are eliminated with the urine and hence are in part waste tissue products. Other aromatic compounds as phenol, indol, skatol, pyrocatechin and probably benzoic acid are not present and are therefore to be considered not as products of tissue metabolism but solely of intestinal putrefaction.

It is evident that glycocoll is an intermediate waste product which would not be met with in the excretions were it not for the presence of cholic or of benzoic acid. In a similar manner the existence of other intermediate waste products has been demonstrated. As already mentioned ammonia is one of these and does not appear in the urine unless mineral acids are administered, or formed by oxidation of proteins in the body. In combination as a salt of a mineral acid it escapes conversion into urea. Carbamic acid is likewise an intermediate waste product which normally is changed into urea. If, however, calcium hydrate is administered calcium carbamate is formed and is excreted. The existence of glycuronic acid as an intermediate waste product was established in a similar manner. When camphor, chloral, naphthol or toluol are administered they unite with glycuronic acid and the resultant compound appears in the urine.

The amount of glycocoll present in the tissues is not unlimited, for when more than about $1 \mathrm{~g}$. of benzoic acid is 
taken some of this acid appears in the urine uncombined. Salicylic acid (hydroxy-benzoic acid) when taken internally appears in the urine as such, and also as salicyluric acid (hydroxy-hippuric acid) OH. $\mathrm{C}_{6} \mathrm{H}_{4} \mathrm{CO} \cdot \mathrm{NH} \cdot \mathrm{CH}_{2} \cdot \mathrm{CO}_{2} \mathrm{H}$. When benzoic acid is given to birds it is not excreted as hippuric acid but as ornithuric acid, $\mathrm{C}_{19} \mathrm{H}_{20} \mathrm{~N}_{2} \mathrm{O}_{4}$. The latter on de. composition yields benzoic acid and ornithin, $\mathrm{C}_{5} \mathrm{H}_{12} \mathrm{~N}_{2} \mathrm{O}_{2}$. Ornithin therefore corresponds to glycocoll and is probably a di-amido-valerianic acid.

The synthesis of benzoic acid and glycocoll to hippuric acid takes place in the kidney.

Herbivorous animals excrete much more hippuric acid than do carnivorous animals. This difference is in part due to the food but more especially to the marked putrefactive changes carried on in the intestines of herbivorous animals. Their intestines are large and pouchy, or sacculate, as compared with the smooth, continuous intestines of carnivorous animals. Consequently the remnants of food will be held back and undergo greater putrefaction. In this way is explained the relatively high excretion by herbivorous animals of such aromatic putrefaction products as phenol, indol, skatol, pyrocatechin, hippuric acid, etc.

Hippuric acid was found in the urine of man for the first time in diabetes. In this disease it is present in increased amount, due undoubtedly to the rich protein diet and to much fruit. In fevers it is decreased and probably also in kidney diseases, because of diminished synthetic activity of the kidney cells. It is not decreased in icterus as has been supposed.

A normal individual, with mixed diet, excretes daily about $0.7 \mathrm{~g}$. of hippuric acid. With a diet rich in fruit the amount may rise to $2.0 \mathrm{~g}$. or more. When present in the sediment in urine it forms long rhombic prisms or needles, and may be mistaken for uric acid, or even for triple phosphates. By attention to diet and to intestinal changes the amount can be promptly diminished. 
Preparation from urine.-Boil $1 / 2-1$ liter of urine (of the horse or cow) with excess of thin milk of lime; filter while hot and concentrate the filtrate on a wire gauze to $1 / 6-1 / 8$ the original volume. Cool, add excess of hydrochloric acid and set aside for 24 hours. Filter off the reddish crystals of hippuric acid and dry them between filter paper. Dissolve the crystals of crude hippuric acid in as little water as possible and filter; heat the filtrate to boiling and pass chlorine gas till the color of the solution becomes pale-yellow. Then cool rapidly, filter and wash the crystals several times with cold water. Recrystallize from boiling water to which animal charcoal has been added (Curtius). To purify the crystals boil again with milk of lime, filter and reprecipitate with hydrochloric acid. The crystals can be still further purified by recrystallization and decoloring with animal charcoal.

Human urine can be employed if $1-1.5 \mathrm{~g}$. of benzoic acid have been taken the previous evening.

Synthetic preparation.-To some glycocoll in a test-tube add a little water, then a few drops of sodium hydrate and about 1 c.c. of benzoyl chloride. Close the tube with a stopper and shake vigorously as long as the tube continues to become hot. Finally render strongly alkaline with sodium hydrate and shake until the odor of benzoyl chloride disappears. When cold acidulate with hydrochloric acid, add an equal volume of ether (petroleum ether is better) and shake up thoroughly in order to extract the benzoic acid. Decant the ethereal layer into a clean porcelain dish and repeat the extraction as before. The aqueous solution with the insoluble hippuric acid is filtered, and the hippuric acid washed slightly on the filter with cold water, then recrystallized from a small amount of boiling water (just sufficient to dissolve).

The ethereal solution on evaporation yields benzoic acid.

The synthesis takes place according to the equation:

$$
\underset{\mathrm{CO}_{2} \mathrm{H}}{\mathrm{CH}_{2} \cdot \mathrm{NH}_{2}}+\mathrm{COCl} \cdot \mathrm{C}_{6} \mathrm{H}_{5}=\underset{\mathrm{CO}_{2} \mathrm{H}}{\mathrm{CH}_{2} \cdot \mathrm{NH} \cdot \mathrm{CO} \cdot \mathrm{C}_{6} \mathrm{H}_{5}+\mathrm{HCl} .}
$$

PROPERTIES OF HIPPURIC ACID.

Determine the melting-point. What is it?

Test the solubility in alcohol, ether and water.

Test the taste and reaction.

Examine the crystalline form under the microscope. 
REACTIONS.

*1.-Carefully neutralize a solution of hippuric acid with sodium hydrate, and add a drop or two of neutral ferric chloride solution when the cream colored ferric salt is precipitated. Test the solubility in hot water, hot alcohol and acids.

2.-Boil a few crystals of hippuric acid with some sodium hypobromite solution-a kermes-brown precipitate forms (distinction from benzoic acid).

3.-Place some hippuric acid in a dish, add nitric acid and evaporate to dryness. Transfer the residue tc a test-tube and heat in a Bunsen flame. Observe the odor of nitro-benzol (artificial oil of bitter almonds).

4.--On moderate heating in a test-tube hippuric acid melts to an oily fluid which on cooling solidifies to a crystalline mass. On stronger heating the liquid becomes red, rises along the walls of the tube, and yields a sublimate of benzoic acid and an odor of hydrocyanic acid (peach-blossoms).

5.-Place $1 \mathrm{~g}$. of hippuric acid in a small Erlenmeyer flask, add about 10 c.c. of dilute sulphuric acid (1-2), then insert a perforated stopper provided with a condensing tube (Fig. 1, p. 10.) Heat the contents on a wire gauze at just below the boiling point for some hours. Observe the sublimation of crystals of benzoic acid. When the decomposition is complete, cool, add about 25 c.c. water and filter off the benzoic acid. Extract the filtrate with ether to remove the dissolved benzoic acid; then dilute the aqueous liquid with water and neutralize on the water-bath with barium carbonate. Filter, concentrate the filtrate on the water-bath almost to dryness, and set aside to allow the glycocoll to crystallize.

Save the benzoic acid for subsequent tests.

Complete the equation:

$$
\mathrm{C}_{6} \mathrm{H}_{5} \cdot \mathrm{CO} \cdot \mathrm{NH} \cdot \mathrm{CH}_{2} \cdot \mathrm{CO}_{2} \mathrm{H}+\mathrm{H}_{2} \mathrm{O}=
$$

\section{Glycocoll.}

To a portion of the glycocoll dissolved in water add a drop of copper chloride-a blue color is the result. What effect has the addition of sodium hydrate to this solution?

To another portion add ferric chloride-note the result. 
Benzoic Acid, $\mathrm{C}_{7} \mathrm{H}_{6} \mathrm{O}_{2},=\mathrm{C}_{8} \mathrm{H}_{5} \cdot \mathrm{CO} . \mathrm{OH}$.

Benzoic acid may be present with hippuric acid in the urine, especially when the glycocoll necessary to make hippuric acid has been all taken up. It may be present, together with uncombined glycocoll, whenever the renal cells are unable to effect the synthesis, as is the case in certain diseases of the kidney. Furthermore, in alkaline urine hippuric acid may be split by ferments into benzoic acid, and it is even possible that a ferment (histozym) exerting such action is present in the kidney of the dog and hog.

The sources of the benzoic acid in the urine have been mentioned under hippuric acid (p. 156).

Preparation.- This acid is obtained in the decomposition of hippuric acid (see p. 160) and can be purified by recrystallization from hot water.

1.-Examine the crystalline form as it is obtained (1) from hot water, (2) from ether.

2.-Heat some benzoic acid in a test-tube, held vertically over the flame-the acid sublimes in needles. Note the odor evolved.

3.-Determine the melting-point of the crystals.

4.-Test the solubility in water, alcohol, and in ether.

5.-To a solution of a salt of benzoic acid add neutral ferric chloride solution. A brownish yellow precipitate forms-distinction from salicylic acid which gives a reddish violet color. To a portion of the precipitate add ammonium hydrate-it dissolves and ferric hydrate is thrown down instead. To another portion add hydrochloric acid and set aside over night. What is the result?

*6.-Place some benzoic acid in an evaporating dish, add nitric acid and evaporate over a flame to dryness; then gently heat-the odor of nitrobenzol (artificial oil of bitter almonds) will be perceived. 
7.-With sodium hypobromite it gives no precipitate-distinction from hippuric acid.

8.-Add a solution of benzoic acid or of its salt to a mixture of alcohol, barium chloride and ammonia. No precipitate forms-distinction from succinic acid.

$$
\text { Indoxyl, } \mathrm{C}_{8} \mathrm{H}_{7} \mathrm{NO},=\mathrm{C}_{6} \mathrm{H}_{4}<\underset{\mathrm{NH}}{\mathrm{C} . \mathrm{OH}}>\mathrm{CH} \text {. }
$$

This occurs in the urine as potassium indoxyl sulphate, $\mathrm{C}_{8} \mathrm{H}_{6} \mathrm{~N}$.O. $\mathrm{SO}_{2} . \mathrm{OK}$, and is commonly known as indican or indigogen.

Indoxyl is derived from indol, which is formed as a product of the putrefaction of protein matter in the intestine. Indol when absorbed becomes oxidized to indoxyl which unites with sulphuric acid and an alkali metal and is then excreted by the kidneys as an ethereal sulphate. Indoxyl may also unite with glycuronic acid and appear thus in the urine.

Indol, skatol and phenol are ordinarily decomposition products due to bacterial activity. They may be prepared by fusing proteins with potassium hydrate. The several color reactions as studied in connection with the proteins (Exp. 6, p. 40) have shown the existence of three distinct aromatic groups or nuclei within the protein molecule. These three are the phenol, phenyl and indol groups. It is evident that certain proteins when acted upon by bacteria will give off the phenol group more readily than either of the other two; or the reverse may occur. The three groups of decomposition products are not always, therefore, in the same relative proportion but may vary within wide limits according to the nature of the bacteria at work and the material being acted upon.

Indoxyl results, as has been stated, by the oxidation of indol. On further oxidation it yields indigo which may therefore be met with in the urine. Indigo on reduc- 
tion yields indol. The amount of indoxyl in normal urine, after a mixed diet, corresponds to from 5 to $20 \mathrm{mg}$. of indigo per day. It is much more abundant in the urine of herbivorous animals, as the horse. This is true not only of indol, but also of skatol, phenol and conjugate sulphates. The reason for this increase of putrefactive products is given under hippuric acid. An exclusively meat diet will, of course, be followed by an increased excretion of these aromatic compounds.

Indoxyl will be increased materially in diseases where the peristaltic action of the intestine is diminished. Likewise in obstructions of the small but not of the large intestine. As is well known from laboratory experience certain bacteria, as the cholera vibrio and the bacillus of typhoid fever, yield an indol reaction. Consequently, in certain infectious diseases of the intestines the amount of indol, and hence of indoxyl, will be increased. This is notably the case in the early stage of cholera. It should be remembered that frequent diarrhoea may diminish the chance of absorption of such decomposition products and hence the urine may contain less aromatic bodies than normal urine. Increased indoxyl excretion is found in typhoid fever, in intestinal tuberculosis, in carcinoma, catarrh and dilatation of the stomach; also in lead colic, in pernicious anæmia and in leukämia. In starvation indoxyl is diminished but phenol may not only persist but may be increased. Bacterial decomposition may continue in the intestines during starvation; the mucin of bile, intestinal secretions, and perhaps intestinal hæmorrhages furnishing material for the bacteria to thrive upon. This material may not give off the indol as readily as the phenol group, and hence the increase in phenol excretion as mentioned.

Indoxyl is a brownish oil which is not permanent but is oxidized, even by the air, to indigo. When heated in the absence of air it yields indoxyl-red. In alkaline urine indoxyl is most likely to undergo conversion into indigo. 
Indigo may occur in urine in solution, in the sediment or in calculi.

Juffe's Test for Indoxyl-To 10-20 c.c. of urine in a test-tube add an equal volume of concentrated hydrochloric acid, a few c.c. of chloroform, then drop by drop a dilute solution of sodium (or calcium) hypochlorite. Shake after the addition of each drop and avoid adding an excess. The chloroform gradually turns blue since the indoxyl has been oxidized to indigo-blue. This reaction has been itilized as the basis of a quantitative method.

$$
2 \mathrm{C}_{8} \mathrm{H}_{7} \mathrm{NO}+2 \mathrm{O}=\mathrm{C}_{16} \mathrm{H}_{10} \mathrm{~N}_{2} \mathrm{O}_{2}+2 \mathrm{H}_{2} \mathrm{O} .
$$

Apply the above test to some urine from the horse or cow, then to human urine.

When the amount of indoxyl or indican is considerable it can be estimated by weight. That is, the indigo which separates out on standing is washed with hot water, ammonia and again with water, then dried at $105^{\circ}-110^{\circ}$ and weighed. It can also be estimated colorimetrically, or with the spectroscope.

Indol itself forms plates which melt at $52^{\circ}$ and are easily soluble in hot water, alcohol, and in ether. It possesses a marked fecal odor. The solution in ligroin gives a beautiful red precipitate with picric acid.

TESTS FOR INDOL.

1. - The solution is colored red by nitric acid containing a trace of nitrous acid. This reaction is not given by skatol.

2.-A pine splinter moistened with hydrochloric acid is colored red on contact with an alcoholic solution of indol.

3.--Millon's reagent gives a yellow color, whereas with skatol it a dirty-brown.

4.-Examine the laboratory specimen of indol-note the odor. 


\section{Skatoxyl, $\quad \mathrm{C}_{9} \mathrm{H}_{9} \mathrm{NO}$.}

The potassium salt of skatoxyl-sulphuric acid, $\mathrm{C}_{9} \mathrm{H}_{8} \mathrm{~N}$. O. $\mathrm{SO}_{2} \mathrm{OK}$, may occur in the urine. Skatoxyl is not known in the free state.

The source of skatoxyl is skatol, which has the same origin as phenol, indol, etc.-namely intestinal putrefaction. The skatol on absorption is oxidized to skatoxyl which unites with sulphuric acid to form an ethereal sulphate, as in the case of phenol and indol. Some skatoxyl may unite with glycuronic acid.

Urines rich in skatoxyl, on standing, color from the surface downward. The color may be reddish, violet or black. Such urine on contact with $\mathrm{HCl}$ gives a dark-red to a violet color; with $\mathrm{HNO}_{3}$ a cherry-red color. Whereas the indigo formed in urine, as in Jaffè's test, is soluble in chloroform or ether, the coloring matter derived from skatoxyl, under the same conditions, is insoluble. It will be dissolved by amyl alcohol, and chloroform; ether will take it up out of neutral or alkaline solutions.

Skatoxyl and indican, in urine, may yield several distinct pigments which have been given various names, such as uroglaucin, urocyanin, urorubin, urohämatin, uroroseïn, etc.

\section{DETECTION OF SKATOXYL.}

1.-To the suspected urine add hydrochloric acid; if skatoxyl is present a dark red to violet color will appear.

2.-Addition of nitric acid to the suspected urine will produce a cherry-red color if skatoxyl is present.

3.- Urine containing much skatoxyl darkens on standing, like "carbolic acid urine," and later becomes reddish, then violet to black.

Skatol, or methyl indol, is a putrefaction product of protein matter. It may be formed direct, or by the reducing action of bacteria on indol. Inasmuch as this reducing action is most marked in the large intestine it 
accounts for the presence of skatol in abundance in that portion of the intestines. An obstruction in the small intestine is indicated by a large increase in indol, whereas an increase in skatol points to the large intestine as the seat of the trouble. The relation of indol, skatol and skatoxyl can be seen in the following formulæ:<smiles>c1ccccc1</smiles><smiles>C=C(C)Nc1ccccc1</smiles><smiles>C=C(CO)NC(=O)c1ccccc1</smiles>

Skatol forms plates which melt at $95^{\circ}$. It is difficultly soluble in water; is soluble in alcohol. The picrate forms red needles. Skatol is soluble in $\mathrm{HCl}$, yielding a violet color. On heating with $\mathrm{H}_{2} \mathrm{SO}_{4}$ it yields a beautiful purplered color. It possesses an intense fecal odor. The synthetic preparation, however, is not so odorous. On boiling with $\mathrm{KOH}$ skatol does not decompose, whereas indol does. Both indol and skatol can be distilled in a current of steam, and are precipitated by picric acid in the presence of hydrochloric acid.

TESTS FOR SKATOL.

1.-It does not give a red color with nitric acid which contains nitrous acid, but only a whitish cloud. acid.

2.-It does not color a pine splinter moistened with hydrochloric

3.-Note the odor of a specimen of skatol.

\section{Phenol and Cresols.}

Phenol, $\mathrm{C}_{6} \mathrm{H}_{5} \mathrm{OH}$, and cresol, $\mathrm{C}_{6} \mathrm{H}_{4} \cdot \mathrm{CH}_{3} \cdot \mathrm{OH}$, are like indol, skatol, etc., common putrefaction products formed in the intestines. When absorbed they combine with sulphuric acid and are eliminated as ethereal salts. If the 
amount of sulphuric acid is insufficient they may unite with glycuronic acid.

The term "phenol" as ordinarily used in connection with urine includes the cresols. The latter are methyl phenols and are present in larger amount than is ordinary phenol. Of the three possible cresols the para-compound is most abundant. The ortho-cresol has been detected in urine. The phenol and cresols are all tested for together.

The source of "phenol" may be preformed aromatic compounds in the food. Vegetable food is especially rich in such compounds and hence increases the amount of these bodies present in the urine. Likewise, the administration of various coal-tar products, as benzol, salol, creosote, will increase phenol, etc., and also conjugate sulphates. Apart from the administration of aromatic compounds as such, or in the food, the only remaining source is the bacterial decomposition of protein matter in the intestine. There is very little evidence that phenol is given off as a tissue product. By the action of the pancreatic ferment, or of bacteria, tyrosin is formed out of the protein molecule and on oxidation will yield phenol.

The amount of phenols present in normal urine is subject to great variation (17 to $51 \mathrm{mg}$.). Increased excretion of phenol is met with in various intestinal diseases wherein bacterial decomposition of the contents is favored. It may be absorbed from large abscesses, as well as from the intestines. It is usually, but not necessarily, associated with indican. Thus, in starvation the amount of the latter is decreased, whereas phenol may be increased. As pointed out under indol, bacterial activity in the intestines is not suppressed during starvation.

Phenol and indoxyl are apparently diminished in nephritis. They are also said to be diminished in the urine in icterus, although conjugate sulphates (probably of other aromatic compounds) are present. It is interesting to note that aromatic substances such as phenol, indican, 
etc., are not increased as a result of abnormal fermentations in the stomach.

Urine rich in phenols takes on a dark brown or dark green color-the so-called "carbolic urine." This is largely due to the presence of products like hydroquinon.

Potassium Phenol Sulphate, $\mathrm{C}_{6} \mathrm{H}_{5}$. O. $\mathrm{SO}_{2}$. OK.

* Synthetic Preparation.-Prepare first some potassium pyrosulphate as follows: To $10 \mathrm{~g}$. of pulverized potassium sulphate in an evaporating dish add $6 \mathrm{~g}$. of concentrated sulphuric acid and warm gently over a flame, stirring well, till the mass dissolves; then gradually raise the heat till the mass remains in quiet fusion. Cool and pulverize.

Now place in a flask $6 \mathrm{~g}$. of potassium hydrate dissolved in 8 to $9 \mathrm{c} . \mathrm{c}$. of water and add $10 \mathrm{~g}$. of phenol. When all is dissolved, cool to $60-70^{\circ}$ and add gradually and in small portions, agitating well, $12.5 \mathrm{~g}$. of the finely pulverized potassium pyrosulphate. Keep the mixture at $60-70^{\circ}$, with frequent shaking, for 8 to 10 hours; then add about 50 c.c. of boiling alcohol, shake thoroughly and filter while hot. The filtrate on cooling solidifies to a mass of bright plates of potassium phenol-sulphate. Recrystallize once or twice from boiling alcohol and finally dry the crystals between filter paper or in a desiccator over sulphuric acid.

The synthesis of this salt is shown by the equation:

$$
\mathrm{C}_{6} \mathrm{H}_{5} \cdot \mathrm{OK}+\mathrm{K}_{2} \mathrm{~S}_{2} \mathrm{O}_{7}=\mathrm{C}_{6} \mathrm{H}_{5} \cdot \mathrm{O} \cdot \mathrm{SO}_{2} \cdot \mathrm{OK}+\mathrm{K}_{2} \mathrm{SO}_{4} \text {. }
$$

*REACTIONS OF POTASSIUM PHENOL SULPHATE.

1.-An aqueous solution of potassium phenol-sulphate is not precipitated by barium chloride even in the presence of acetic acid. Why not? The commercial salt will give a precipitate owing to the presence of sulphates.

2.-To an aqueous solution of the salt add hydrochloric acid and heat a few minutes, then add barium chloride. 
What is the result? Write the equation to represent the change.

3.- In solutions of the salt test for phenol with brominewater, ferric chloride, and Millon's reagent. What is the result?

4.-Distill a solution of the same, acidulated with hydrochloric acid, and in the distillate test for phenol.

\section{TESTS FOR PHENOL.}

1.---To a dilute solution of phenol add a drop of neutral ferric chloride solution-violet color.

2.-To some phenol solution add bromine-water to a permanent yellow color-yellowish white precipitate of crystals of tri-bromphenol, $\mathrm{C}_{6} \mathrm{H}_{2} \mathrm{Br}_{3} \mathrm{OH}$. Examine under the microscope.

3.-To the phenol solution add some Millon's reagent and warm till the precipitate dissolves-beautiful red color. Explain the behavior of proteins with Millon's reagent.

Detection of Phenol in the Urine.-To about 250 c.c. of horse's or cow's urine add 25 c.c. of sulphuric acid and distil. Examine the distillate by the above tests for phenol (and cresol), and for aceton, applying the tests given on page 178 .

\section{Pyrocatechin, $\mathrm{C}_{6} \mathrm{H}_{4}(\mathrm{OH})_{2}$.}

This is ortho-dioxybenzol $(1: 2)$ and occurs in the urine as pyrocatechin sulphate, $\mathrm{C}_{6} \mathrm{H}_{4} \mathrm{O}_{2}\left(\mathrm{KO} . \mathrm{SO}_{2}\right)_{2}$.

It is present usually in small quantity and may be entirely absent. It is more abundant in horse urine. The protocatechuic acid present in plant food may be considered as its chief source, although it may form in the body by the incomplete oxidation of phenol. Consequently the administration of phenol, benzol, etc., increases the amount of pyrocatechin in the urine. It has been reported in transsudates, and is probably present in the supra-renals. According to Halliburton it is present in the cerebro-spinal 
fluid, but this has not been confirmed by.Nawratski who found the reducing substance to be probably glucose $(0.05$ per cent.)

It has been frequently observed that urine, at first of normal color on standing, especially if alkaline, turns brown and even black. This change begins at the surface and gradually extends downward. This condition of urine has been designated as alkaptonuria. In some instances at least this may be due to pyrocatechin; in others to uroleucinic, or more often perhaps to homogentisinic acid. Such urine has the additional peculiarity that it reduces Fehling's solution, and may therefore be mistaken for diabetic urine.

Pyrocatechin crystallizes from water, or ether in prisms; from benzol in broad plates. It melts at $104^{\circ}$ and sublimes in glistening plates. It is soluble in water, alcohol, and in ether; also in cold benzol (distinction from hydroquinon). It is precipitated by lead acetate.

With a solution of pyrocatechin make the following tests:

1.-To a few c.c. of an aqueous solution add several drops of potassium hydrate solution; set aside and notice the change that takes place in the course of an hour. What is this change due to? Add some pyrocatechin to a little urine and repeat the test.

2.-To the dilute aqueous solution add some ferric chloride. The solution becomes dark green, then black; now add ammonium hydrate to alkaline reaction. What is the result? Acidify with acetic acid and the original color returns.

3. - To about 1 c.c. of the aqueous solution add one drop of furfurolwater, then add slowly 1 c.c. of concentrated sulphuric acid in such a way that it runs down the side of the tube and collects at the bottom. The liquid becomes cherry-red in color, later violet.

4.-Boil with a little Fehling's solution. Observe the reduction. Solutions of other metals, such as silver, gold, and platinum are likewise reduced, whereas bismuth is not. 
*Detection of di-oxy-benzols in urine.-The urine is acidulated with $\mathrm{H}_{2} \mathrm{SO}_{4}$ and concentrated to expel phenol, then filtered. The cool filtrate is repeatedly extracted with ether. The ethereal extracts are combined and distilled; the residue is neutralized with $\mathrm{BaCO}_{3}$ and again extracted with ether. The ether solution is evaporated, the residue dissolved in water and treated with lead acetate, avoiding excess. The precipitate contains pyrocatechin; the filtrate, hydro-quinon. The washed precipitate is suspended in water, acidulated with $\mathrm{H}_{2} \mathrm{SO}_{4}$ and extracted with ether. This, evaporated and recrystallized from benzol, gives pyrocatechin. The filtrate is treated in similar manner and the final residue recrystallized from hot benzol gives hydroquinon.

Hydroquinon, $\mathrm{C}_{6} \mathrm{H}_{4}(\mathrm{OH})_{2}$.

This is para-dioxybenzol $(1: 4)$. It is not a normal constituent but may be found in urine after administration of benzol, phenol, or hydroquinon as an ethereal sulphate. Such urine on exposure to air eventually turns dark-giving the so-called carbol-urine.

Hydroquinon crystallizes in rhombic prisms or plates which melt at $169^{\circ}$. The solubility is much the same as that of pyrocatechin. It is, however, soluble in hot benzol and is not precipitated by lead acetate. Like pyrocatechin it reduces alkaline solutions of metals. It does not, however, reduce bismuth subnitrate.

Test a little hydroquinon solution:

1.-According to the directions given above under (1) and ( $t$ ).

2.-Rapidly heat a minute portion in a test-tube. Observe a violet vapor which condenses to an indigo-blue sublimate.

3.- Heat some of the solution with ferric chloride. It is oxidized and the penetrating odor of quinon is observed. 
Cystin, $\quad\left(\mathrm{C}_{3} \mathrm{H}_{6} \mathrm{NSO}_{2}\right)_{2},=\mathrm{CH}_{3}-\mathrm{C}_{\mathrm{CO}_{2} \mathrm{H}}^{\mathrm{C}}-\mathrm{NH}_{2} \mathrm{NH}_{2}-\left.\right|_{\mathrm{CO}_{2} \mathrm{H}} ^{\mathrm{C}}-\mathrm{CH}_{3}$.

Cystin is an organic sulphur compound present in the urine in the rare condition known as cystinuria. It is not present in normal urine, though a cystin-like substance has been found in very small amounts. Normally, the sulphur of the protein molecule, after passing through a number of intermediate stages or compounds, is in large part completely oxidized and eliminated as sulphuric acid. In cystinuria nearly one-quarter of the total sulphur may appear in the urine as cystin. It may therefore be considered as a product of abnormal cell metabolism-an intermediate waste product that has escaped complete oxidation. That such intermediate compounds exist is demonstrated by feeding brom-benzol to dogs, in which case a substituted cystein appears in the urine. The benzol compound unites with cysteïn and protects it against oxidation in the same way that benzoic acid protects glycocoll. Cysteïn is $\alpha$-amido-thiolactic acid.<smiles>CC(N)(S)C(=O)O</smiles>

On oxidation it yields cystin, and the latter in turn on reduction with nascent hydrogen gives rise to cysteïn. When cystein is fed to an animal it is oxidized in the body and the sulphur is eliminated in part, or wholly, as a sulphate, and it is because of this proneness to oxidation that cystein is not present in normal urine.

In cystinuria the urine and feces have been shown to contain one or both of the well known ptomains, cadaverin 
and putrescin. These bases are known to be bacterial products and formed therefore, in the intestine. They are not present in the urine or in the feces of normal persons nor are they present in the excreta of diseased individuals except in cholera. It would seem, therefore, as if a special organism were present in the intestines in cystinuria, and that its products, the diamines, have to do with the excretion of cystin. The latter is not present in the feces. It is a product of cell metabolism within the body. It is probable that this strange association of diamines and cystin is due to a protecting action of the former, whereby the latter escapes oxidation. The diamines and other products are made in the intestines by bacteria; they are absorbed and unite with cystin, and this combination as soon as it is excreted by the kidneys is decomposed into cystin and diamines.

In cystinuria the urine is usually alkaline or very slightly acid. Cystin when present in urine, owing to its difficult solubility, will be found in the sediment and may even give rise to the rare cystin stones. No other pathological change is observed in cystinuria. The condition has been met with more often in men than in women, and moreover has been known to occur in several members of the same family. Apart from its presence in the urine in cystinuria, cystin has been found once in beef kidneys, in the liver of a horse, and of a drunkard, and in the pancre. atic digestion of fibrin.

Cystin is strongly lævo-rotary. On reduction with tin and hydrochloric acid it yields cysteïn, which may be precipitated quantitatively by mercuric chloride. Cystin crystallizes in characteristic colorless, thin six-sided plates. In addition to its microscopical appearance cystin can further be recognized by the following properties:

1.-It is insoluble in water, alcohol, ether, acetic acid; soluble in mineral acids and alkalis. 
2.-In alkaline concentrated solution with benzoyl chloride (see p. 133) it gives benzoyl cystin. This method can be used for separating cystin that is dissolved in the urine.

3.-On boiling with potassium hydrate the sulphide of potassium forms and may be recognized by bringing it in contact with a bright silver coin, or by adding a drop of lead acetate.

\section{4.-It yields no murexid test (p. 145).}

Examination of cystin calculi.-Pulverize and dissolve in sodium carbonate solution and then precipitate this with acetic acid. Collect the precipitate, wash, and dissolve in a little ammonia. Set this solution aside in a watch-glass to evaporate spontaneously, then examine microscopically.

\section{Diamines.}

Cadaverin, $\mathrm{C}_{5} \mathrm{H}_{14} \mathrm{~N}_{2}=\mathrm{NH}_{2} \cdot \mathrm{CH}_{2} \cdot \mathrm{CH}_{2} \cdot \mathrm{CH}_{2} \cdot \mathrm{CH}_{2} \cdot \mathrm{CH}_{2} \cdot \mathrm{NH}_{2}$. Putrescin, $\mathrm{C}_{4} \mathrm{H}_{12} \mathrm{~N}_{2}=\mathrm{NH}_{2} \cdot \mathrm{CH}_{2} \cdot \mathrm{CH}_{2} \cdot \mathrm{CH}_{2} \cdot \mathrm{CH}_{2} \cdot \mathrm{NH}_{2}$.

These two diamines were discovered in 1885 by Brieger. They are basic products formed in the bacterial decomposition of various proteins and consequently belong to the group of ptomains. They are usually found together, and their presence in the urine and feces in cystinuria indicates intestinal origin. They have been found, but not constantly, in the discharges of cholera and cholerine. They are not present in normal urine.

These bases can be isolated from the urine of cystinuria by means of the benzoyl chloride reaction. For the method and other details see, Vaughan and Novy, Ptomaïns, etc., 1896, p. 325 . 


\section{Leucin and Tyrosin.}

These amido acids are not present in normal urine but may be present in pathological urines, in solution, or, if the amount is considerable, in the sediment. Because of its greater insolubility tyrosin is more likely to occur in the sediment. The urine may contain these compounds in various liver diseases, as in acute yellow atrophy and in phosphorus poisoning; also in typhus fever, small-pox, and in severe anæmia. For isolation and identification see pages 83 to 86 .

$$
\text { Oxalic Acid, } \mathrm{C}_{2} \mathrm{H}_{2} \mathrm{O}_{4},=\begin{aligned}
& \mathrm{CO} . \mathrm{OH} \\
& \mathrm{CO} . \mathrm{OH}
\end{aligned}
$$

This compound occurs in the urine as calcium oxalate, and although present in small amounts it is nevertheless a constant constituent of normal urine. On an average about $20 \mathrm{mg}$. are excreted per day. The source of this oxalic acid is not clearly understood. When oxalic acid or its salts are administered it is in part eliminated as such. Since many articles of food, especially vegetables, as lettuce, spinach, asparagus, tomatoes, apples, grapes, etc., contain this acid, it is commonly held that the oxalic acid of normal urine is derived from that contained in the food. The administration of remedies like rhubarb, scilla, senna, valerian, etc., for like reason increase the amount of oxalic acid. On the other hand it is ascribed to an incomplete oxidation of carbohydrates, fats and proteins and even of uric acid.

When oxalic acid is unduly increased in amount this condition is designated as oxaluria. This is met with in diabetes where the presence of oxalic acid, as well as of sugar, is ascribed to a lack of oxidation. It is also met with in icterus, in cases where there is diminished meta. bolism, digestive or nervous disturbances. At times it 
may be present in urine, in excess, without any other noticeable symptom. No special signficance is attached to small amounts of oxalates. The chief danger lies in the formation of stones, in the kidney or in the bladder.

Oxalates may be present in acid, neutral or alkaline urine. They are at first held in solution by the acid phosphates. On standing and cooling these are changed to neutral phosphates and oxalates are deposited. Calcium oxalate usually forms small bright octahedra; it may form dumb-bells, discs, or may be amorphous. Its insolubility in acetic acid distinguishes it from phosphates.

Preparation of calcium oxalate.-To about 200 c.c. of urine add a few drops of saturated oxalic acid solution, then set aside for 24 hours. Examine the sediment under the microscope for the characteristic octahedral crystals, and for other forms.

1.- Sketch the different forms of calcium oxalate.

2.-Test the solubility in water, acetic acid, hydrochloric acid.

Aceton, $\mathrm{CH}_{3} \cdot \mathrm{CO} . \mathrm{CH}_{3}$.

Aceton, or di-methyl keton, is probably a constant constituent of urine. The amount present in normal urine is about $10 \mathrm{mg}$. per day. It may be considerably increased in certain diseases and this condition is designated as acetonuria. An increased excretion of aceton may be expected whenever there is increased protein disintegration. Thus, it is increased in starvation, or after a meat diet. A carbohydrate diet, because of its saving action on proteins, will diminish aceton. It may be increased to ten, or even forty times the normal amount, in diabetes; it is also increased in febrile diseases, and in many wasting diseases, as cachexia, anæmia, carcinoma, etc. It is increased by chloroform narcosis, and by administration of salacetol. 
Aceton is also met with as a product of fermentation of carbohydrates in the alimentary tract. Thus, it may be found in lactic acid fermentations in the stomach, and in the intestines. Hence it may be in the feces. It has been found in febrile blood and in the breath.

The chief source of aceton is without doubt the disintegrating protein molecule. One of the early products formed is $\beta$-oxy-butyric acid, which may be oxidized to acetacetic acid, and this in turn yields aceton. All three of these substances may be present at the same time, as in

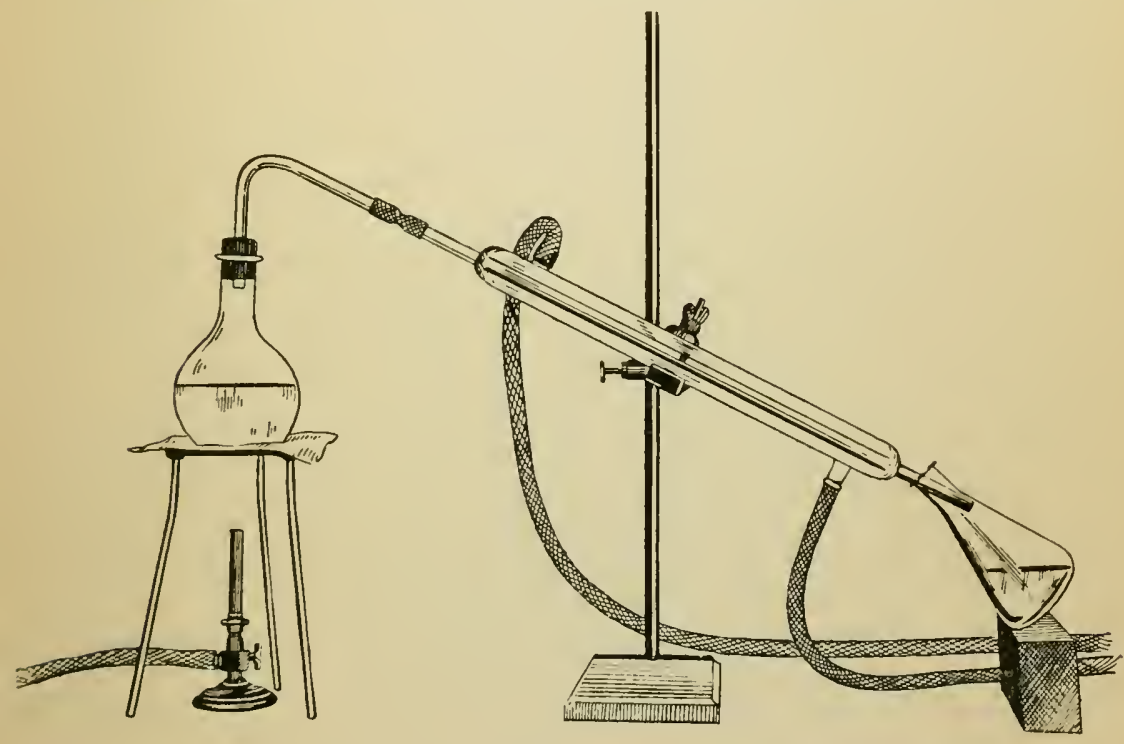

Fig. 3.

diabetes. In diabetes 2 to 5 or even $10 \mathrm{~g}$. of aceton may be excreted per day.

Aceton itself is a liquid which boils at $56-57^{\circ}$. It possesses a decided-fruit-like odor and is soluble in water, alcohol, and ether. It is easily oxidized to acetic and formic acids, and with Ehrlich's reagent gives a red color.

Isolation of Aceton. - As a rule aceton cannot be tested 
for directly in the urine. It is necessary to resort to distillation. For this purpose 250 c.c. of fresh urine (preferably diabetic) are acidulated with dilute acetic acid and .20-30 c.c. of liquid are distilled off (Fig. 3). The distillate is then subjected to the following tests:

1.-Lieben's iodoform test.-To the aceton solution add some iodine in potassium iodide, then potassium hydrate till the iodine just clears off-a yellowish-white cloud results. Allow to settle, then examine for iodoform crystals-six-sided plates or stellate groups.

To a few c.c. of water add some alcohol and test for iodoform as just given.

Inasmuch as alcohol may be present in urine the test as given is not positive. The following modification distinguishes between the two compounds.

2.-Gunning's test.-Render the liquid strongly alkaline with ammonia, then add tincture of iodine, drop by drop, till the black precipitate of nitrogen iodide forms. On standing this disappears more or less rapidly and iodoform crystals remain if aceton is present.

3.-Legal's nitroprusside test.-To the liquid to be examined add a few drops of freshly prepared sodium nitroprusside, then some caustic alkali. A ruby-red color similar to that given by creatinin appears. On acidulating with acetic acid, the color is changed to wine-red if aceton is present, and to yellow if it is absent. If ammonia is used instead of caustic alkali aceton will give the color reaction, whereas creatinin will not.

4.-Penzoldt's indigo test.-Dissolve a few crystals of ortho-nitrobenzaldehyde in hot water; cool, add the liquid to be tested and then some sodium hydrate. If aceton is present the liquid turns yellow, then green and finally blue. On shaking with chloroform the blue color is taken up.

5.-To some mercuric chloride solution add alcoholic potash. A precipitate of the oxide forms. Then add the aceton solution, shake thoroughly and filter. Test the filtrate with ammonium sulphide for mercury. Aceton dissolves freshly precipitated mercuric oxide. 
Acetacetic Acid, $\mathrm{C}_{4} \mathrm{H}_{6} \mathrm{O}_{3},=\mathrm{CH}_{3} \cdot \mathrm{CO} \cdot \mathrm{CH}_{2} \cdot \mathrm{CO}_{2} \mathrm{H}$.

This compound is probably not present in normal wrine. It is not as common in febrile urine as aceton. It may be present in the urine in chronic diseases, as tuberculosis. Like aceton it is formed, though not as constantly, by the breaking down of protein matter. It is frequently present in diabetes (diaceturia).

Acetacetic, or diacetic, acid is a colorless liquid soluble in water, alcohol and ether. On heating with water it readily decomposes into carbonic acid and aceton. Hence in the distillation of urine the aceton that is found may in part be derived from the breaking down of this compound.

1.-To $10-50$ c.c. of the urine (diabetic) add dilute ferric chloride as long as a precipitate of phosphates forms, filter and to the filtrate add some more ferric chloride-a wine-red color indicates acetacetic acid. (Gerhurdt's test).

2.- The urine on distillation yields aceton.

3.-Mömer"s test. To the urine add some potassium iodide solution, then ferric chloride in excess. If diacetic acid is present, on boiling vapors are given off which are intensely irritating to the eyes and nose.

It should be remembered that many aromatic compounds like antipyrin, salicylic acid, etc., give a similar reaction with iron. The reaction if due to diacetic acid is not given by the urine if this is allowed to stand for a day or two, or if it is heated.

\section{\%-Oxy-butyric acid, $\mathrm{C}_{4} \mathrm{H}_{8} \mathrm{O}_{3}$.}

This acid is absent from normal urine. It may be present in the urine of acute infectious diseases, and in diabetes. In the latter disease it may be present to the amount of $30-50 \mathrm{~g}$. per day, and in one case as much as $226 \mathrm{~g}$. were found. It is combined with ammonia. 


\section{Cholesterin.}

As stated on p. 95 cholesterin is a very rare constituent of urine. It may be expected when fat is present in the urine, as in chyluria. For its recognition and isolation see the page mentioned.

Fats.

Fats may be present in the urine as globules, or as needle-shaped crystals. The nature of these crystals can be readily ascertained by gently heating a specimen on a slide when, if composed of fat, they melt, forming globules. The solubility of the globules in ether will at once distinguish these from leucin. It should be remembered that fat is a frequent accidental constituent of urine, as when urine is collected in an unclean vessel, or when passed after digital examination, or after the use of a catheter. Pathologically fat may be present in urine as a result of fatty degeneration of the kidney, etc. This condition (lipuria) is met with in many acute infectious diseases, in Bright's disease, in phosphorus poisoning, etc. Epithelial cells and casts may be expected at the same time. Fat may be present in the urine in pregnancy.

Fat is frequently met with in the urine, in the tropics and sub-tropics, in the condition known as chyluria. Such urine may be milk-white in appearance, or may be colored red by blood admixture. Albumose, pepton, cholesterin, etc., have been found in such urine.

Fatty acids, such as formic, acetic, butyric are apparently present in normal urine in small amount $(50 \mathrm{mg}$. per day). They are increased after a carbohydrate diet, in fevers (lipaciduria), in structural diseases of the liver, as in cancer, or syphilis. They are considerably increased during ammoniacal fermentation of. urine. 
Fats may constitute the nucleus of urinary calculi, and in rare cases such calculi may consist of only fat and fatty acids (Horbaczewski).

\section{Carbohydrates.}

Urine, normal and abnormal, may contain a number of carbohydrates. Thus, glucose is a constant constituent of normal urine; the amount, however, rarely exceeds 0.02 per cent. and is therefore not recognizable by ordinary tests. Iso-maltose and inosite are probably present. A dextrin-like body known as animal-gum has also been found in normal urine.

Under special conditions other carbohydrates may appear. This is seen, for instance, when large quantities of glucose (200 g.) are ingested. In a few hours sugar can be detected in the urine, but it soon disappears. The same is true when lævulose, or cane sugar, or lactose are taken in large amounts. These may appear, therefore, as such in urine. Lactose, moreover, may be present in the urine in pregnancy. Fruit rich in pectic substances (pentosanes) as cherries, plums, etc., may cause the appearance of pentoses.

An increase in carbohydrates in general can be detected in the urine by the application of Molisch's reaction (Exp. 1, page 17); also by the benzoyl chloride reaction.

\section{Pentoses.}

These sugars, as mentioned above, may be present in the urine after certain fruit diet. They have been found in several urines (p. 16, pentosuria); in diabetes, and as decomposition products of a nucleo-proteid from the pancreas. Such urine will give a slow reduction on prolonged heating with Fehling's solution, but will not undergo fer- 
mentation if glucose is absent. The separation of pentoses from glucose can be accomplished by preparing the osazons (p. 21). On digesting the mixed osazons with water at $60^{\circ}$ the pentosazon dissolves while the glucosazon is insoluble. The former can then be recrystallized; it melts at $157-160^{\circ}$. The reaction of pentoses with anilin acetate is given on p. 16. Another reaction, likewise based upon the formation of furfurol, is to add to fuming $\mathrm{HCl}$, saturated with phlorogucin, one-half its volume of urine. The mixture is immersed in boiling water. The foam and liquid become colored an intense red. This reaction, it should be remembered, is also given by glycuronic acid.

\section{Glucose.}

As already stated glucose is present in traces in normal urine. When, however, it is increased beyond this amount and is constant in the urine it becomes abnormal (glycosuria). Sugar may thus be increased under various pathological conditions, as in lesions of the brain and cord; in diseases of the heart, liver and lungs; in cholera; in various intoxications as from morphine, chloral, carbon monoxide, etc.

Persistent excretion of sugar is met with in diabetes mellitus. The amount of sugar excreted in this condition may vary from a trace to one-half or even one kilogram in 24 hour's urine. The quantity of the urine is greatly increased, 3-5 litres, or more per day. The color of the urine is therefore usually very pale. The absolute amount of urea and of other normal constituents is likewise greatly increased. Consequently, although the quantity of the urine is considerable, the presence of the sugar and the increase in the other solids impart a high specific gravity to the urine. This is commonly $1.030-1.040$ but may be much higher.

The excretion of a large quantity of urine which possesses a very light color, a high specific gravity, and reduces 
Fehling's solution points to the presence of sugar. It should be remembered that normal urine may give a slight reduction, because of the presence of reducing sugars, or of uric acid and creatinin, or of glycuronic acid compounds. The latter may appear in the urine in increased amount, as conjugate compounds, after administration of camphor, chloral hydrate, etc. Furthermore, in alkaptonuria there are reducing substances as uroleucinic and homogentisinic acids. Reducing substances also appear in the urine after administration of rhubarb, senna, antipyrin, salol, turpentine, etc. The reaction of urine with copper or bismuth solutions does not prove that sugar is present; it merely indicates the presence of a reducing substance which may, or may not, be sugar. If the reaction is negative one can correctly conclude that sugar is absent. The fermentation test, or osazon reaction (p. 21), can be em. ployed to prove that the reducing substance is glucose.

Fehling's test (Exp. 8 a, p. 19) is commonly employed for the detection of sugar. Albumin may be present in diabetes. Aceton, acetacetic acid, oxy-butyric and fatty acids are also present. Diabetic urine on standing will frequently contain yeast-cells.

\section{Albumin and Globulin.}

Normal urine does not contain these or other proteins, except mucin, which is probably a nucleo-albumin. Serum albumin and serum globulin, at times even fibrinogen, may appear in the urine under a variety of conditions. These compounds are all included under the term "albumin." The relative amount of these substances is not constant; at times the urine may contain chiefly albumin, and again globulin may predominate. Apparently albumin predominates when diuresis is marked, and when this is decreased globulin is increased. The quantity of albumin and globulin varies greatly from the merest trace, indicated by faint cloud- 
iness when acid is added, to such quantities as will cause the urine to solidify on heating. Usually the amount excreted in a day is a fraction of a gram, but may be increased to 20 or even 30 grams. It is, however, usually less than $x / 2$ per cent. This relatively small amount, taken into consideration with the increased urine excretion (polyuria) explains why the specific gravity of urine in albuminuria is low, 1.010 or even less. When the secretion of urine is diminished, as may happen in the later stages of nephritis, the specific gravity rises and may reach 1.040 or more.

Temporary albuminuria may occur in otherwise healthy. persons as a result of improper food, nervous disturbances and excessive muscular exercise. In the latter case the protein present is probably a nucleo-albumin since it is precipitated by acetic acid in the cold. The presence of blood, pus, and semen will introduce albumin into urine and such origin is detected by the aid of the microscope. It may also occur in pregnancy.

Albuminuria proper is most often the result of parenchymatous degeneration of the kidney, and is accompanied by casts. It occurs in acute or chronic nephritis, in intoxications from substances such as arsenic, phosphorus, strychnine and especially bacterial toxins. Hence febrile albuminuria is a common condition in acute infectious diseases as pneumonia, erysipelas, scarlet fever, small-pox, yellow fever, malaria, diphtheria, etc. Usually the albuminuria disappears with the fever. In such cases casts are present and the number of leucocytes in the sediment is increased. Slight albuminuria has been met with in ulcer of the stomach; in icteric urine, where it is accompanied by pale yellow hyaline casts and by a nucleo-albumin; in acute yellow atrophy of the liver; in anæmia and at times in diabetes, carcinoma and in rheumatism; also in dyspnoic conditions due to respiratory and circulatory disturbances.

Detection.-Before applying the tests for albumin the 
urine, if not clear, should be filtered. Otherwise a slight cloudiness resulting from the application of a test may escape unnoticed. Should the urine be very concentrated it will be well to dilute it with several parts of water, or better with dilute salt solution, to prevent precipitation of uric acid, or of urea nitrate.

The best test for albumin is the nitric acid and heat test as given in Exp. $9 a$, p. 43. It is really Heller's test supplemented by heat whereby the precipitate or cloudiness due to urates, nucleo-albumin, etc., is dissolved. After administration of balsam of tolu or copaiba the urine may give with nitric acid an opalescent ring, soluble, however, in alcohol. It should be remembered that the presence of salt favors the precipitation of albumin.

The ferrocyanide test is likewise very delicate. Any mucin or nucleo-albumin present in the urine should first be removed by acidulation with acetic acid, allowing to stand for some time, then filtering. To the clear filtrate the reagent can then be added (p. 43).

The separation of albumin and globulin is accomplished by saturation with magnesium sulphate (p. 48). 25-50 c.c. or more of the urine should be taken.

Estimation.-For the quantitative estimation of albumin and globulin see Chapter XI.

\section{Albumose.}

This substance, or rather group of hydrated proteins, may at times be present in urine. The "pepton" that has been met with in urine is undoubtedly an albumose. Such pepton, or rather albumose, may be given off by disintegrating leucocytes as in large abscesses; it becomes absorbed and is then promptly excreted. The recognition of "pepton" in urine in case of a deep-seated abscess is impor- 
tant. Albumose frequently appears in the urine during pregnancy and during confinement.

Albumoses have been found, though not constantly, in febrile diseases, such as the acute infectious diseases; in cancer and ulcer of the stomach; in acute yellow atrophy of the liver and after phosphorus poisoning; in anæmia and leukæmia; and in acute rheumatism.

Typical albumosuria is very rare and thus far only about six cases have been described. In nearly all of these softening of the bones (osteomalacia) existed. The crystalline globulin found in urine in the sediment and described by Bramwell and Patton is in reality, according to Huppert, hetero-albumose. Albumose had been met with once before in the sediment of urine.

Detection.-Only fresh urine should be tested for albumose since albuminous urine may on standing, by the action of ferments, give rise to albumose. Urine rich in albumin should always be tested for albumose. Albumose coagulates at about $60^{\circ}$ and the precipitate redissolves on boiling, whereas albumin coagulates at about $70^{\circ}$ and does not redișsolve on boiling.

The method for the detection of albumose is given under Exp. 3, p. 50. Also compare methods given under pepton.

\section{Pepton.}

What has been termed pepton by the older writers is in reality albumose. There is probably no positive evidence that true pepton has been met with in fresh urine. According to Siegfried anti-pepton has the formula $\mathrm{C}_{10} \mathrm{H}_{15} \mathrm{~N}_{3} \mathrm{O}_{5}$ and is identical with the "fleisch-säure" which he isolated from meat extracts. 
Histon.

This substance, which resembles in many respects pepton, was first obtained by Kossel from the nuclei of blood corpuscles of birds. Subsequently it was studied by Lilienfeld who obtained it by decomposing the nucleo-histon derived from lymphocytes. With ammonia it gives an insoluble precipitate and it is coagulated by boiling. Histon has been reported in peritonitis; in the later stages of pneumonia, erysipelas, scarlet fever, and in leukæmia.

Detection.-The urine is precipitated with alcohol; the precipitate is washed with hot alcohol and dissolved in boiling water. This solution is cooled, acidulated with hydrochloric acid and allowed to stand for several hours, then filtered. To the filtrate ammonia is added; the precipitate is filtered off and washed with ammonia till the wash-water ceases to give the biuret reaction. The precipitate is dissolved in acetic acid and tested (1) by biuret; (2) by coagulation.

\section{Nucleo-albumin and Mucin.}

The nucleo-albumins are soluble in water, if alkali is present, and from such solution they are precipitated on saturation with magnesium sulphate (distinction from nucleo-histon). In this respect they resemble globulin, but they contain phosphorus and on treatment with pepsin and hydrochloric acid yield nuclein. They are thrown out of solution by acetic acid but are soluble in excess of the reagent (distinction from mucin).

Both mucin and nucleo-albumin are probably present in minute quantity in normal urine. The mucin frequently separates from the urine on standing as a very delicate cloud. Mucin is detected by adding acetic acid to the urine previously diluted with two or three volumes of water. If 
necessary the urine should first be filtered. It is soluble in excess of mineral acid. It is insoluble in excess of acetic acid and on decomposition gives a reducing substance (see page 59)-distinction from nucleo-albumin and nucleo-histon. Heller's test may give a reaction with mucin which might be mistaken for albumin. Mucin is especially present in the urine of women, and is increased in affections of the mucous membrane of the urinary passages.

Nucleo-albumin, often called mucin, is increased in the urine of febrile diseases; in leukrmia, catarrhal icterus, and after severe muscular exercise.

Fibrin.

Fibrin may be present in the sediment of urine as threads, flakes or as blood casts. It may be recognized by the presence of blood cells, and by its insolubility in cold dilute acids and alkalis; it is soluble on prolonged heating (p. 113).

\section{Blood.}

As a result of hemorrhage in the kidneys, or elsewhere along the urinary tract, blood may appear in the urine. This condition is designated as hematuria. The amount of blood may be so small as to scarcely alter the color of the urine. On the other hand the urine may be bright red, in which case it will necessarily be cloudy. It should be remembered that large amounts of urates may give a reddish, cloudy appearance. Microscopic examination will easily establish the presence of blood cells and hence of hamaturia. In addition to the corpuscles, fibrin floccules, or even blood casts, may be found. Albumin and globulin will, of course, be present in such urine.

As a rule the microscope is all that is necessary to establish the presence of blood in the urine. The guajac 
test (p. 105) and Heller's test supplemented by the hamin test (p. 105) may be used. The latter will be griven also in hamoglobinuria.

The place of hamorrhage may be indicated by the form of the blood clot. Thus, if narrow blood casts are present, they point to the hidney as the seat of the himorrliage, especially if the amount of blood is slight. Large, coarse blood clots clearly are not formed in the kidney. The presence of squamous epithelial cells and absence of casts point to the bladder or urethri.

\section{Hæmoglobin .}

At times the urine may be blood-red in color and yet not due to actual haemorrlage. Normally the bloodpigment, hamoglobin, is held within the corpuscles. If, howerer, destruction of the cells takes place, as in extensire burns, or intoxication with arsine, chlorates, etc, the hamoglobin is set free and dissolves in the plasma. It is then a foreign substance, as much so as if it were injected into the blood-current, and is consequently excreted by the liver and by the kidneys. The presence of hamoglobin in solution in the urine is designated as hemoglobimuria. Not infrequently the hiemoglobin may be converted into methamoglobin.

This condition is recognized by the color of the urine, the absence of blood cells, by a positive reaction with guajac (p. 105), and by the spectroscope (p. 102).

\section{Hæmatoporphyrin.}

This pigment may be spolien of as hematin deprived of its iron. It is said to be present in normal urine in very minute quantity. It has been found in the mine in rheumatism, Addison's disease, cirrhosis of the liver, ete.; and 
especially is it present in the urine after continued administration of sulphonal or trional. Such urine may be dark, or brownish red, or may have even a violet tinge. If the amount of coloring matter is small the urine may scarcely show a color.

Detection.-To 25 c.c. of the urine, baryta mixture or sodium hydrate is added to alkaline reaction. The phosphates are precipitated and drag down the hæmatoporphyrin. The precipitate is washed, then digested at room temperature with alcohol acidulated with hydrochloric acid and filtered. The filtrate is examined before the spectroscope for the characteristic spectrum of acid hæmatoporphyrin (see Exp. 5, p. 103).

The origin of this compound is uncertain. According to Stokvis blood that passes into the intestines is there reduced to hæmatoporphyrin which is then absorbed and excreted by the kidneys.

\section{Methæmoglobin.}

This modified blood pigment is formed in the urine from hæmoglobin. It is present quite often in hæmoglobinuria. It is recognized by the spectroscope, but care must be taken not to confound it with hæmatin, (see Exp. 4, p. 103). The change in the spectrum after addition of ammonium sulphide distinguishes it from hæmatin.

\section{Pus.}

Normal urine may frequently contain occasional leucocytes. As a result, however, of various inflammatory conditions in the kidneys, bladder or urethra pus may appear in variable quantity (pyuria). If formed in the kidney or bladder it becomes distributed throughout the 
urine and consequently the last portion of urine that is passed will be as rich in pus corpuscles as the first portion. In urethral affections, however, the first portion of the urine will contain nearly all the pus cells and the last portion will be almost free of such cells.

The presence of pus in urine is often indicated by an abundant, slimy sediment. Such urine always contains albumin.

1.-Microscopic Examination.-This is the quickest and best way for the detection of pus. The pus cells are larger than blood corpuscles; they are colorless, and round or crenated. One or more nuclei can be seen, especially if acetic acid is applied. With iodine in potassium iodide the nuclei turn a mahogany brown. In alkaline urine the contents of the cell (nucleo-histon) are largely dissolved out and hence the cell itself and the nuclei may be scarcely recognizable. Such urine on the addition of acetic acid will give a precipitate of nucleo-histon. A slimy sediment, together with albumin, is therefore indicative of pus.

2.-Donne's test.-To the sediment obtained on standing, or by centrifugation, add a small piece of potash and stir. A very slimy, sticky mass results.

3.-Vitali's guajac test.-Cover the sediment, acidulated if need be, with a layer of guajac tincture. A blue coloration develops. Or, filter through a small filter, then pass through the filter a few drops of the guajac tincture. The paper and filtrate turn blue.

\section{Nucleo-histon.}

This substance has been shown by Lilienfeld to be the chief constituent of the nuclei of the cells of the thymus gland and other tissues. On treatment with acids and other agents it readily splits up into nuclein and histon. The complex character of nucleo-histon can best be seen from the following scheme, which shows at the same time the successive products of decomposition: 


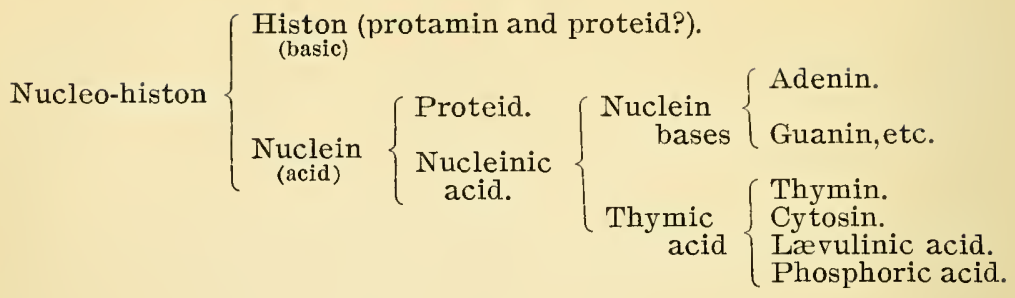

Nucleo-histon has been isolated from the urine in " case of pseudo-leukæmia (Jolles). It may be always expected in alkaline urine containing pus. When present in urine it is precipitated on the addition of acetic acid and can therefore be mistaken for nucleo-albumin. If the precipitate, however, is dissolved in dilute sodium carbonate and the solution saturated with magnesium sulphate the nucleoalbumin is precipitated, whereas the nucleo-histon remains in solution. That the precipitate is not mucin can be demonstrated by the absence of reducing power after decomposition (as in Exp. 13 b, p. 59).

To isolate nucleo-histon, a large volume of urine is heated at $60-70^{\circ}$ for $1 / 4$ hour, then filtered. The filtrate is cautiously acidulated with acetic acid and then thoroughly shaken to cause the flocculent precipitate to settle. The precipitate is filtered off, dissolved in dilute sodium hydrate (4 per cent.) and filtered. The filtrate is precipitated with acetic acid as before, and this purification is repeated a third time. The precipitate is finally shaken with an equal volume of absolute alcohol, filtered, washed with warm alcohol, then with ether and finally dried.

To identify the product, treat it for some hours with 1 per cent. hydrochloric acid in the cold and then filter. Histon will be present in the filtrate and can be recognized (1) by giving a precipitate on the addition of ammonia; (2) by coagulating on heating, the precipitate being soluble in acids; (3) by giving the biuret reaction in the cold. 


\section{Bile.}

The bile acids and bile pigments, which constitute the characteristic constituents of bile, are elaborated in the liver and under normal conditions they are passed with the bile into the intestines. As a result of intoxication with phosphorus, arsenic, bacterial toxins, etc., or of closure of the bile duct by catarrhal exudates, bile stones, etc., the constituents of the bile pass into the blood. The pigments in this case induce the condition of jaundice, or icterus. The bile constituents in the blood are in part taken up by the kidneys and eliminated with the urine.

The bile acids probably do not exist, even in traces, in normal urine. They may be present in icteric urine, though this is not always the case. The small amount of these acids when present, and the organic constituents of the urine render it difficult to detect these acids in urine. The method for their detection in urine is given under Exp. 6 c, p. 91.

The bile pigments impart more or less color to the urine. It may be yellowish green to brown, and on shaking a yellowish or greenish foam results. Such urine may be cloudy and may contain albumin; colored casts or epithelial cells and granules may be in the sediment. Bilirubin has been identified in such urine. Other bile pigments which are easily derived from bilirubin may also be present. The tests given under Exp. 7, p. 92, are delicate and can be employed with satisfactory results.

\section{Ehrlich's Diazo-reaction for Typhoid Urine.}

The reagent employed for this reaction should be freshly prepared. The following two solutions are first made.

1. - To 1,000 c.c. of water add 50 c.c. of concentrated $\mathrm{HCl}$ and $1 \mathrm{~g}$. of sulphanilic acid. 
2.-A 0.5 per cent. solution of sodium nitrite. The nitrite solution is subject to oxidation on standing, and should not therefore be prepared in large quantity.

Just before use, to form the reagent proper, these two solutions are mixed as follows: To 250 c.c. of solution No. 1 add 5 c.c. of solution No. 2. Or, on a smaller scale, to 5 c.c. of No. 1 add 3 or 4 drops of solution No. 2.

Mix the urine with an equal volume of the reagent, and add at at once an excess of $\mathrm{NH}_{4} \mathrm{OH}$. A pink to a deep red color, and especially a pink colored foam, constitutes the diazo-reaction.

Normal urine, as a rule, gives a brownish yellow, very rarely a pinkish color. The reaction is very rare in chronic non-febrile diseases. It is met with, as a rule, except in very light cases, in typhoid fever, and a certain diagnostic value is therefore ascribed to this reaction. It has been found, however, in exanthemic typhus, in smallpox, in acute miliary tubeculosis, in severe tuberculosis, and in pneumonia. The disappearance of the reaction in typhoid urine may be taken as a favorable sign, while the appearance of the reaction in tuberculosis is an unfavorable indication.

The substance which gives this reaction is unknown. It is an aromatic compound, probably a metabolic product which appears in the urine only under certain special conditions.

The reaction resembles somewhat the test for nitrites. as given in Exp. 11, p. 58. If naphthylamin is replaced by $\alpha-n a p h t h o l$ the reaction is even more similar.

\section{Sulphur.}

The proteins of the food and of the tissues constitute almost the sole source of the sulphur-containing waste products. A small amount of waste sulphur compounds is. eliminated as sulphocyanate by the saliva, gastric juice, 
etc. Another small portion leaves the body as taurin in the taurocholic acid of bile. With these exceptions almost all the sulphur resulting from protein disintegration appears in the urine. For the sake of convenience the sulphur compounds of the urine are divided into two groups: (1) oxidized or acid sulphur; (2) unoxidized or neutral sul. phur.

The first group, or oxidized sulphur, contains compounds in which the sulphur is present as sulphuric acid, $\mathrm{SO}_{3}$. This is the chief form in which sulphur is excreted. Thus, while the total sulphur in a day's urine may be as high as $1.0 \mathrm{~g}$., the sulphur present as $\mathrm{SO}_{3}$ averages $0.8 \mathrm{~g}$. $\left(2 \mathrm{~g} . \mathrm{SO}_{3}\right)$. This group is in turn sub-divided into $(a)$ simple sulphates, such as potassium, sodium, magnesium, calcium sulphates; (b) conjugate, or ethereal sulphates. In the latter the sulphuric acid is in combination with an organic radical such as phenol, indol, skatol, etc. The former is precipitated by $\mathrm{BaCl}_{2}$, the latter is not. Calcium sulphate may appear in the sediment as bundles of long needles not unlike those of tyrosin. The relative amounts of these two groups is expressed by an average ratio of 10 to 1 . Consequently the 24 hours' urine contains $0.1-0.2 \mathrm{~g}$. of $\mathrm{SO}_{3}$ as conjugate sulphate.

The source of the sulphur in the sulphates, as indicated above, is the sulphur contained in the proteins of the food and of the tissues. Inasmuch as the sulphates con. tain most of the waste sulphur it follows that the total sulphates in the urine furnish an excellent index of protein disintegration. On the other hand the organic radicals, such as phenol, indol, etc., present as conjugate sulphates owe their origin either to the administration of aromatic bodies such as creosote, carbolic acid, etc.; or to the intestinal putrefaction of proteins. The protein of the food may undergo bacterial decomposition in the intestines, and phenol, indol and other aromatic compounds may thus be 
formed. These aromatic compounds are probably not formed in the breaking down of the tissue proteins. Consequently the amount of sulphur present as conjugate sulphates indicates the extent of intestinal putrefaction. Conjugate sulphates are present in the urine of herbivorous animals in large amount. They are increased in the urine of man by intestinal obstruction, as in constipation, and are decreased after cathartics and in starvation.

The second group, unoxidized or neutral sulphur compounds, includes all the sulphur bodies except sulphuric acid. Some of these compounds, as thiosulphates and sulphocyanates are easily oxidizable, whereas others like taurin, are difficultly oxidizable. As included under the head of neutral sulphur may be mentioned, also hydrogen sulphide, cystin, cystin-like bodies, sulphonic acids, ethyl sulphide, mucin, etc. The neutral sulphur in the urine of man averages about 15 per cent. of the total sulphur. In the urine of the dog it is more than double this amount.

\section{Hydrogen Sulphide.}

It is possible for this compound to appear in the urine, eliminated directly from the blood. In such a case, severe intoxication necessarily exists and death results. A second source of hydrogen sulphide is the decomposition that may exist in neighboring pus cavities; diffusion takes place through the walls of the bladder and the gas is absorbed by the urine. In both instances the urine is likely to be perfectly clear. On the other hand, if hydrogen sulphide is present and the urine is cloudy it indicates a fermentative decomposition of the urine analogous to the ammoniacal fermentation. In this case bacteria have been introduced into the bladder through a rectal fistula, or by a catheter. They decompose certain of the neutral sulphur compounds and yield hydrogen sulphide. Several micrococci 
and bacilli have been isolated from such hydrogen sulphide urine (hydrothionuria). This property belongs not merely to a few organisms, but rather to most bacteria. Thus, a large number of the common non-pathogenic and pathogenic germs will give rise to this gas when grown in urine.

Hydrogen sulphide can be detected by its odor and by its blackening a bright silver coin. A much more delicate test is to suspend in the neck of the bottle, from a slit in a cork or from a cotton plug, a strip of filter paper previously dipped in an alkaline lead acetate solution. Blackening promptly results if the gas is present.

\section{Sulphuric Acid, $\mathrm{H}_{2} \mathrm{SO}_{4}$.}

To about 10 c.c. of urine in a test-tube add some acetic acid, then some barium chloride solution-barium sulphate is precipitated. Now filter and to the filtrate add hydrochloric acid (1 c.c.); boil for a few minutes, and set aside. Another precipitate forms. What is it? Explain the reaction.

The experiment shows that sulphuric acid exists in the urine in at least two forms-as ordinary sulphate, and as ethereal sulphate.

Write the equations:

$$
\begin{aligned}
& \mathrm{BaCl}_{2}+\mathrm{K}_{2} \mathrm{SO}_{4}= \\
& \mathrm{C}_{6} \mathrm{H}_{5} \cdot \mathrm{O} \cdot \mathrm{SO}_{2} \cdot \mathrm{OK}+\mathrm{H}_{2} \mathrm{O}=\mathrm{KHSO}_{4}+
\end{aligned}
$$

Prepare and examine under the microscope crystals of calcium sulphate, made by adding a few drops of calcium chloride to some urine and allowing it to stand for a short time.

* "Neutral" sulphur can be tested for as follows:

Filter off the barium sulphate precipitate which forms in the hydrochloric acid solution as described above and from the filtrate remove the barium by careful addition of sodium carbonate solution. Filter, evaporate the filtrate to dryness, and fuse the residue with potassium nitrate and hydrate. Dissolve the fused mass when cold in water, acidulate with hydrochloric acid and precipitate with bar- 
ium chloride; the neutral sulphur has been oxidized to sulphuric acid. If the quantity of urine taken be known, and the weight of the precipitate be determined, the amount of neutral sulphur can be estimated.

Hyposulphurous (Thiosulphuric) Acid, $\mathrm{H}_{2} \mathrm{~S}_{2} \mathrm{O}_{3}$.

Make the following tests with a solution of sodium hyposulphite:

1.-Warm some of the solution with hydrochloric acid-notice the odor, and the cloudiness due to separation of free sulphur.

Complete the equation:

$$
\mathrm{Na}_{2} \mathrm{~S}_{2} \mathrm{O}_{3}+2 \mathrm{HCl}=
$$

This test may be applied to urine suspected of containing hyposulphurous acid, as that of a cat, or dog. The milky appearance on standing is due to free sulphur.

2.-To a few c.c. of the solution add a few drops of silver nitrate solution. The solution on standing becomes black from reduced silver.

3.-Add 1 or 2 drops of neutral ferric chloride to some of the solution. What is the result?

4.-To some hyposulphite solution add barium chloride, then alcohol-the difficulty soluble barium salt forms.

\section{Phosphoric Acid, $\mathrm{H}_{3} \mathrm{PO}_{4}$.}

This acid exists in the urine in combination with sodium, ammonium, potassium (alkaline phosphates) and with calcium and magnesium (earthy phosphates). A small amount of phosphoric acid may exist in ethereal combination as glycerin-phosphoric acid, or as lecithin.

Phosphoric acid $\left(\mathrm{H}_{3} \mathrm{PO}_{4}\right)$ forms three series of salts:

Normal- $\mathrm{M}_{3} \mathrm{PO}_{4}$.

Mono-hydric- $\mathrm{M}_{2} \mathrm{HPO}_{4}$.

Di-hydric- $\mathrm{MH}_{2} \mathrm{PO}_{4}$. 
The latter salt is acid in reaction, and as $\mathrm{NaH}_{2} \mathrm{PO}_{4}$ it is present in urine and to it the acidity of this secretion is largely due. Sixty per cent. of the total phosphorus in urine exists as a di-hydric salt.

The sources of phosphoric acid are: first, the preformed phosphates in the food; second, the phosphorus in organic combination as proteins, nucleins, lecithin, etc. Excess of earthy bases in the food may result in precipitation of the phosphates in the intestine, in which case they are excreted with the feces, and hence the urine will be poor in phos. phates. This is true in the case of herbivorous animals.

The quantity of phosphoric acid in the urine is therefore subject to considerable variation. On an average 2.5 g. of $\mathrm{P}_{2} \mathrm{O}_{5}$ are excreted per day. About two-thirds of this amount is combined with alkali metals, the remainder with earthy bases. The earthy phosphates are held in solution by the acid phosphates and by salt; when the reaction becomes neutral or alkaline they are precipitated. Excessive muscular exercise is attended with increase of phosphoric acid.

The phosphoric acid is diminished in amount in febrile affections such as the acute infectious diseases; also in diseases of the kidneys, because of non-elimination. It is increased in meningitis and in diabetes.

\section{Neutral Calcium Phospnate, $\mathrm{Ca}_{3}\left(\mathrm{PO}_{4}\right)_{2}$.}

1.-Heat some urine in a test-tube. Observe that it becomes cloudy; add a drop of nitric acid and the cloudiness dissolves. What is it due to?

2. -To some urine add sodium hydrate; a precipitate of the phosphates of calcium and magnesium is thrown down. Examine the precipitate under the microscope. What is its appearance? Test the solubility of the precipitate in acetic, hydrochloric and nitric acids. How would you distinguish between a deposit of amorphous phosphates, amorphous urates, and amorphous oxalates? 
* Acid Phosphate of Calcium, CaHPO ${ }_{4}$-To a solution of calcium chloride add some di-sodium hydric phosphate solution, drop by drop. Examine carefully the characteristic form of the crystals. This salt is always crystalline and is deposited in slightly acid urine only.

To some acid urine add dilute ammonium hydrate till only a faint acid reaction remains. Set aside till crystals form, then examine under the microscope.

\section{Magnesium Ammonium Phosphate, $\mathrm{MgNH}_{4} \mathrm{PO}_{4}$.}

TRIPLE PHOSPHATE,

1. - To some urine add ammonium hydrate and set aside over night. Examine under the microscope for stellate or pennate crystals of triple phosphate.

2.-Set some urine aside for a few days. Ammoniacal fermentation sets in and the prismatic form of triple phosphate is deposited. Examine the characteristic crystals.

This salt is not deposited in the urine unless ammonia is present. The reaction may be neutral or alkaline. If stellate crystals of triple phosphate are found in a urine what does it indicate? Are they of importance? When prismatic crystals of triple phosphate are found what does it show? When are they of importance?

How would you distinguish the short prismatic form of triple phosphate from crystals of oxalate of lime?

\section{Chlorides.}

Sodium chloride is the chief form in which hydrochloric acid exists in the urine. The other bases combine with but relatively small amounts of this acid. It is probable that a variable amount of chlorine (10-40 per cent.) exists in an organic combination.

The amount of chlorides in the urine depends primarily upon the quantity of these salts contained in the food. Furthermore, increased exercise, and ingestion of water are followed by increased excretion of chlorides. The 
amount of sodium chloride excreted by an adult in 24 hours will necessarily vary greatly. It is usually $10-15 \mathrm{~g}$.

Chlorides may be markedly decreased in acute febrile conditions; likewise in diarrhoea, or when large exudates or transudates form. On the other hand chlorides are increased after crises, or when exudates are being absorbed. A decrease in chlorides is met with, further, when there is lack of absorption on the part of the stomach or intestines; or when there is insufficient excretion by the kidneys.

The chlorides, because of their solubility, are never met with in the sediment in urine.

\section{Urinary Sediment.}

Normal acid urine, at the time of passage, is usually a perfectly clear solution, free from visible suspended or insoluble matter. If the urine is neutral or alkaline in reaction it will usually be cloudy owing to the precipitation of phosphates. A cloudy acid urine, due to suspended bacteria, is met with in acid fermentation, as in hydrothionuria. The normal acid urine, on cooling, may undergo a change in reaction, due to the fact that owing to mass action di-hydric phosphates take the sodium away from the urates and thus form mono-hydric phosphates which impart a less acid or even neutral reaction to the urine. As a result of the withdrawal of sodium free uric acid is deposited. It should be remembered that about $0.7 \mathrm{~g}$. of uric acid may be held in solution in the urine by the urea and the acid phos. phates. From normal urine, when allowed to stand for some time, there invariably separates a light cloud of mucin which may contain a few mucous corpuscles. Urine may therefore be perfectly clear when passed and may give rise to a sediment on subsequent cooling and standing; or, the urine may be cloudy from suspended matter at the time of passage. 
The suspended matter in urine may consist of a great variety of substances, many of which possess great diagnostic importance. In order to make a microscopic exam-

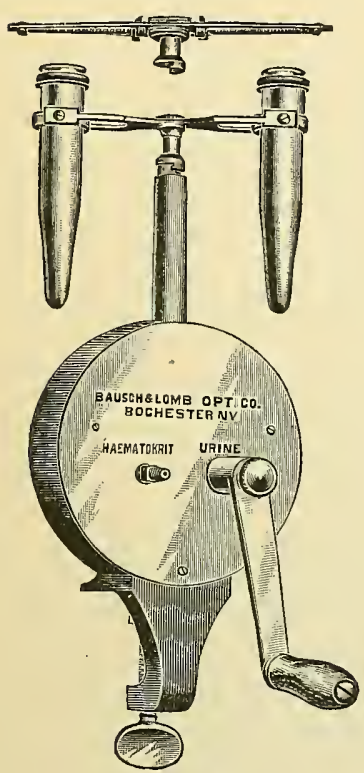

Fig, 4. ination it is necessary to allow the urine to stand for some hours, as over night, in a conical test-glass. The suspended matter then settles to the bottom and can be removed with a pipette. Much time can be gained and better results obtained by the used of some one of the numerous forms of hand centrifuges (Fig. 4). The suspended matter can thus be sedimented in 2-3 minutes. In making microscopic examinations one should be on guard and be able to recognize the presence of foreign material, such as fatty globules or crystals; starch granules; cotton, linen, wool or silk threads; hair and the like.

It is convenient to divide the substances that may be present in the sediment into two groups,-organized and unorganized.

\section{Organized Sediment.}

Under this head are included those substances which are made up of cells; that is to say they are organized. Casts are described under this head although they are not, strictly speaking, organized.

Epithelial cells may be expected in small numbers in every urine. The large, flat squamous cells are especially met with in the urine of woman. Epithelial cells from different parts of the urinary tract may be present in large 
numbers. Their form and size may often indicate their origin. Thus, the squamous and cylindrical forms may be derived from the urethra, vagina, or bladder. Numerous small, roundish cells, frequently grouped and present in albuminous urine, are derived from the uriniferous tubules. In alkaline urine the cells may undergo partial solution and are therefore less distinct. They can be stained with dilute anilin dyes.

Mucous corpirscles and wandering leucocytes can be expect. ed in small numbers in normal urine. With iodine solution the nuclei take on a mahogany brown color (glycogen reaction).

Pus corpuscles, or altered leucocytes, when present in appreciable numbers, indicate an acute or chronic inflammatory condition of some part of the urinary tract. The cells are round and usually have 2 or 3 nuclei which are rendered distinct by the application of acetic acid. In alkaline urine the contents dissolve more or less, and render the liquid slimy. This is often the case in cystitis. Albumin always accompanies pus corpuscles in urine. The pus may be derived from the urethra, from the bladder, or from the kidneys. For further tests see p. 191.

Blood corpuscles will be present in the urine whenever there is a hæmorrhage in some portion of the tract. Albumin and globulin are therefore likewise present. This condition, hæmaturia, is to be distinguished from hæmoglobinuria. An abundant deposit of urates may impart a reddish color to the sediment. The cause of hæmorrhage is to be ascertained if possible. In some cases, as in tropical hæmaturia, the cause may be an animal parasite; in others the tubercle bacillus, or other organism may be the causal factor. The place of hæmorrhage may often be indicated by the form of the clots, or blood casts, as they are called. Long, slender blood casts accompanied by 
renal epithelial cells would indicate that the trouble is in the kidney.

Tissue débris, or irregular masses of cells, are often met with in cancer of the bladder; less often in cancer of the kidney; likewise in tubercular affections of these organs, or when animal parasites are present. In such cases blood is frequently present, or the blood pigment may be altered and appear as the yellow rhombic plates of hæmatoïdin. Furthermore, bacterial invasion may exist, hence pus corpuscles and putrid decomposition are often present. The recognition of a "cancer cell" in the urinary sediment is not possible.

Spermatozoa may be present in urine in spermatorrhœea. They possess active motion and can easily be recognized by the microscope. They can be stained by the ordinary anilin dyes. When present in urine this may be more or less cloudy and will show mucous-like threads. The recognition of spermatozoa is of considerable medico-legal importance in suspected criminal coition.

Casts.-As a result of inflammatory changes the epithelium lining the uriniferous tubules gives off or allows the passage of exudative products which solidify in the tubule and thus form a cast. Eventually the pressure of liquid forces the cast out of the tube and it is then carried away with the urine. Casts are usually found in albuminous urine. It should be remembered, however, that casts may exist in urine without albumin being present. This is not infrequently the case after prolonged exercise, such as bicycling. It is customary to distinguish between hyaline, granular, epithelial and vaxy or amyloid casts.

Hyaline casts are colorless, transparent, homogeneous. They are usually narrow, but may be wide and have a wavy border. Owing to their transparent, colorless appearance they may be easily overlooked. The diaphragm should 
therefore be constricted so as to shut off most of the light. They may be rendered more visible by the application of a dilute anilin dye. With iodine they color yellow. They are soluble in acetic acid and in this respect are different from mucin threads. When present in icterus they may have a yellowish color. They may be present in severe fevers, accompanied by albumin. They may also be present in urine when no albumin or epithelial cells can be detected.

Granular casts are rather variable in size and color. They may be considered as hyaline casts in which granular detritus has become imbedded. They are grayish to yellowish in color.

Epithelial casts are merely one or the other of the above forms which are covered in part or entirely by epithelial cells. The inflammatory process has loosened the epithelial cells, which adhere to the cast more firmly than to the basement membrane and hence are eventually expelled adherent to the cast.

Waxy or amyloid casts are comparatively rare. They are more refractive than the hyaline casts and have a dull yellowish, homogeneous appearance. They are usually rather broad and have a wavy, twisted contour. They consist of amyloid and are more resistant to acids than the preceding. Treated with iodine they take on a reddish brown color, and if previously exposed to sulphuric acid they turn violet (amyloid reaction). They are met with in several forms of nephritis, and in contracted and amyloid kidneys.

In addition to the types of casts as just described various modifications, or mixed casts, are met with. These may be briefly alluded to. 
Blood casts, or clots formed within the tubules, are met with in acute nephritis. The blood corpuscles are held in the fibrin meshes or are cemented by albuminous matter. The cast may then be part hyaline and part blood. Long blood casts are not infrequently mistaken for worms.

Pus or leucocyte casts may likewise result from admixture of these cells with a hyaline matrix.

Fatty casts are usually hyaline cylinders containing fatty globules or bunches of needle-shaped crystals of fatty acids.

False or pseudo-casts are not infrequently met with in urine and must be distinguished from the preceding since they have an entirely different origin. Such casts may consist of mucous threads, or of mucous threads in which urates have become imbedded; or of masses of bacteria (zoöglœa threads). Cholesterin and uric acid may also occur in cast-like form. The mucous filaments are rather wide and often branch, and are insoluble in acetic acid; hence readily distinguishable from hyaline casts.

Cylindroids such as are found in cholera, scarlet and recurrent fever, etc., resemble somewhat hyaline casts. They are very long, flat bands with frayed ends.

Vegetable Organisms.-Bacteria are not present in normal urine. When such urine, however, is collected in ordinary vessels and, moreover, is exposed to the air, bacteria are soon introduced and by their activity induce decomposition. The previously clear urine becomes cloudy due to the suspended bacteria and possibly to a change in the reaction. The most common change thus induced is the ammoniacal fermentation whereby urea undergoes hydration and yields ammonia and carbonic acid. The power of inducing fermentation of this type belongs not to one species 
but to a large number of pathogenic and non-pathogenic bacteria. Some of these are micrococci, others are bacilli; sarcine forms may be present. The term micrococcus ureæ, of Pasteur, does not denote one species but is rather to be regarded as a group name for these organisms. No clinical importance is attached to such decompositions when they take place after the urine has been passed. It not infrequently happens, however, that bacteria of this kind have entered, or have been introduced into the bladder where they grow and induce exactly the same change as that which occurs in normal urine on standing. Such urine, contaminated within the body, will be cloudy at the time of passage and is neutral or ammoniacal in reaction.

Other accidental bacteria introduced in a similar way may induce hydrogen sulphide fermentation (hydrothionuria, p. 196). Such urine is cloudy, acid in reaction, and has the odor of rotten eggs.

Certain pathogenic bacteria may be present in urine and their recognition is consequently a matter of considerable importance. The urine is centrifugated and the deposit is examined according to the methods described in text-books on bacteriology.

Tubercle bacilli may be present in the urine in tubercular affections of the kidney, bladder, prostate, etc. When urine persistently contains small quantities of pus or blood it should be repeatedly examined for these organisms. Care should be taken not to confound the smegma bacillus with the tubercle bacillus.

The gonococcus ordinarily will be found in the first portion of urine that is passed since this disease is located in the urethra. The disease may extend inward so that the last as well as the first portion of the urine will be rich in pus corpuscles and in gonococci.

In abscesses of the kidney, the urine may contain staphylococci and streptococci. In severe infections, as in 
typhoid fever, anthrax, etc., the specific organisms may appear in the urine.

The necessity of using sterile catheters to prevent infection should be clearly understood.

Yeast cells are common in diabetic urines, especially when these have stood for some time. The recognition of yeast cells offers no difficulties.

Moulds may develop on the surface of urine on standing. The greenish growth of penicillium glaucum is most common. The fungus of lumpy-jaw, actinomyces, although not strictly belonging under this head may be mentioned as having been found in urine.

Animal Organisms.-Parasitic organisms of this class are not infrequently found in the urine in tropical countries. They are of much less common. occurrence in the higher latitudes, and even in such cases a previous residence in a warm climate may often be established. Infection with these animal parasites may take place either from the blood, through the kidneys, or from the exterior. The constant presence of a little blood (tropical homaturia), and of small aggregations of epithelial cells point to the possible presence of such organisms. Some of the so-called worms found in urine may be merely altered blood clots.

The blood may bring to the kidneys echinococci, the larval form of the tape-worm; eggs of Distomum hæmatobium; or embryos of the Filaria sanguinis.

Organisms that are found at times in the intestines or in the vagina may also be met with in urine. Such are the Oxyuris vermicularis, Cercomonas and Trichomonas vaginalis. The latter has been found but a few times in urine, once by Dock. 


\section{Unorganized Sediment.}

Under this head are described definite chemical compounds, which may either be amorphous or may form perfect crystals. They are unorganized since they do not possess cell structure. These chemical substances are usually difficultly soluble acids, or difficultly soluble salts.

Uric acid exists in the urine in solution, as a urate, at the time of passage. On cooling, as a result of mass action, the acid phosphates appropriate the base, sodium, and hence free uric acid crystallizes out. On heating the urine the precipitate of uric acid will redissolve. The crystals are invariably reddish-yellow or brown in color and may vary greatly in form. They may be identified by the form and color; solubility in alkalis, piperazin, or lysidin; and by the murexid reaction.

Urates may deposit in febrile, or in concentrated urines. They are usually colored pink or red and hence the deposit might be mistaken for blood. On heating the urine the urates promptly dissolve. On the addition of an acid to the amorphous urate deposit crystalline uric acid forms. The potassium and sodium urates, as well as uric acid, are met with in acid urines. In neutral or ammoniacal urine the urate of ammonium may deposit and can be readily recognized by the burr-like form.

In order to apply the murexid test the sediment should be removed by filtration, washed with water, then with alcohol, and finally transferred to a dish and the test applied (Exp. 6, p. 145).

Hippuric acid is very rare in the deposit in the urine of man. It deposits under similar conditions as uric acid. The prismatic crystals likewise color a yellowish-red and may be mistaken for the rarer forms of uric acid. The solubility 
in alcohol, etc., and the absence of the murexid reaction will serve to distinguish it from uric acid.

Cystin forms thin, colorless six-sided plates. It is a very rare constituent and may give rise to stones.

Tyrosin may form bundles of slender needles. Leucin is more likely to be in solution but on concentration it forms characteristic spherical masses.

Calcium oxalate may appear in alkaline as well as acid urine. It usually forms characteristic octahedra, but may exist as dumb-bells, discs or may even be amorphous. The latter can be distinguished from phosphates and urates by its behavior to acetic acid (insoluble); it is soluble in hydrochloric acid. Oxalates, in small numbers, are present in normal urine.

Calcium carbonate is especially met with in the alkaline, cloudy urine of herbivorous animals. It occurs amorphous, or as granules, or dumb-bells. It is rare in the urine of man.

Calcium sulphate is likewise a rare constituent of sediments. It forms long, colorless prisms, or bunches of needles not unlike those of tyrosin. It is insoluble in acetic acid and in ammonia, and can thus be distinguished from tyrosin.

Phosphates are deposited usually in alkaline urine. If the alkalinity is due to potassium or sodium carbonate the phosphates of calcium and of magnesium are precipitated in an amorphous form. 'In this condition they are readily washed out of the bladder. If the urine is ammoniacal the crystalline ammonium magnesium phosphate, commonly known as triple phośphate, will deposit. The urine may be neutral or even slightly acid and yet triple phosphates may form, provided some ammonia is present. The crystalline 
form, the reaction of the urine, the presence of ammonium urate and the behavior with acetic acid will enable identification.

The normal magnesium phosphate may occur in rhombic plates, but this form is very rare; usually it is amorphous. The acid phosphate of calcium may likewise form crystals in faintly acid urine.

Cholesterin crystals may float on the surface of the urine or may be present in the sediment. The same is true of fats and fatty crystals.

Albumoses. - Certain albumose bodies may be present in the sediment in amorphous, or in crystalline form. Crystalline proteins are very rare and in only one case have they been observed in urine (p. 186).

Xanthin crystals are likewise exceedingly rare. They may form xanthin stones.

Indigo may deposit as dark blue stellate needles in alkaline urine which is rich in indoxyl. These crystals may float on the surface of the urine or may be found in the sediment.

Bilirubin has been met with in acid urine as amorphous yellow granules or as plates imbedded in mucus. It is a very rare constituent. For recognition the Gmelin test can be applied under the microscope.

\section{Urinary Calculi.}

Calculi, or stones, may be formed in the kidneys or in the bladder. Any one of the numerous substances which may be present in the sediment may enter into the composition of a urinary calculus. If the urine is acid in reaction the calculus will consist of substances that deposit in acid urine. On the other hand, if the urine is 
alkaline, fixed or ammoniacal, corresponding compounds, such as neutral phosphates, or triple phosphate will be present in the stone. When the stone is formed in undecomposed urine, having an acid, or fixed alkaline reaction, it is said to be of primary formation. But when organisms invade the bladder and set up ammoniacal fermentation the characteristic deposit of ammonium urate, triple phosphate, etc., ensues. The stones that are formed in this case are spoken of as secondary.

In order that a calculus may form, whether it be in the kidney, bladder, liver or elsewhere, it is necessary that some insoluble substance be present to serve as a nucleus, around which layer after layer, or crystal on crystal, can be deposited. Just as a crystal of sugar or piece of thread placed in a thick syrup serves to start the crystallization or deposition of the sugar, so a crystal of uric acid, or of triple phosphate, or a piece of fat may serve as the starting point in the formation of a urinary calculus. Uric acid and urates are by far the most common substances which form the nuclei of stones-about 81 per cent. Less than 9 per cent. of the calculi begin with a nucleus of earthy phosphates, and less than 6 per cent. begin with one of calcium oxalate. Cases are known where tallow or paraffin introduced by catheters, or by injection, constituted the nucleus. In other instances, blood clots, or mucous threads served the same purpose. Usually there is but one nucleus in a stone, although two or more may be present.

Urinary calculi may be divided into simple and composite. The simple stones are rare and consist of but one chemical substance. Thus, we may have calcium oxalate, cystin, xanthin, fat, or cholesterin stones.

The composite stones are much more common. In these there are usually several substances arranged in layers. Thus, a layer of uric acid may be followed by one of urates, this by phosphates, oxalates, and the like. Varia- 
tion in the reaction of the urine will bring about different deposits, and may even result in the removal of substances already deposited. Thus, a uric acid layer may be followed by earthy phosphates, or may even be replaced by these, if the urine possesses fixed alkaline reaction. If subsequently ammoniacal decomposition sets in triple phosphate, ammonium urate, etc., may be thrown down.

Stones may vary in size from that of a pea to that of an egg. When the accretions are small and numerous they are passed with the urine and are spoken of as gravel.

Uric acid calculi are by far the most common and consist either of the free acid or of urates. They are invariably colored yellowish to dark red. The surface may be smooth or rough, and when broken a concentric arrangement may usually be seen. The uric acid may alternate with oxalates.

Ammonium urate stones are smaller, softer and more crumbling than the preceding. They are rare as primary stones (in children); common as secondary stones.

Calcium oxalate yields perhaps the hardest stones. They are distinctly crystalline and not infrequently the octahedral crystals can be made out by the eye. The sharp pointed ends of these crystals impart a very rough surface to the almost colorless stone and may cause hæmorrhages and severe pain if passed by the urethra.

Phosphatic calculi are commonly secondary and are mixtures of phosphates (normal and triple), carbonates, urates and oxalates. They may attain considerable size. In color they are usually white, gray or yellow and they have a more or less chalky character. Simple stones of triple phosphate, or of acid phosphate of calcium, are rare.

Calcium carbonate stones are rare in man but common in herbivorous animals. 
Cystin calculi are likewise very rare, occurring only in cystinuria. They have a pale yellow color and a smooth, soft surface. They are primary in origin and may attain the size of an egg.

Xanthin stones are exceedingly rare and vary greatly in size. They are usually brownish in color and on rubbing they take on a waxy appearance.

Urostealiths. - This term is applied to very rare calculi which consist almost entirely of fat or rather of fatty acids, either free, or combined with calcium and magnesium. They are light, soft and have a brownish color.

Calculi of fibrin or of coagulated blood, and of mucus have also been met with.

Cholesterin stones. - Although these are common in bile, they are extremely rare in urine. Indeed, only one instance is known. The same is true of Indigo calculi.

\section{Examination of Urinary Calculi.}

The following method of analysis taken from Hammarsten will serve as a guide in such examinations. If the stone shows distinct layers, each one of these should be tested by itself:

Heat a portion of the powdered stone on a platinum foil.

A. It does not burn.

The original powder treated with $\mathrm{HCl}$.

(a). Effervesces.-Calcium carbonate.

(b). Does not effervesce. A portion of the powder is gently ignited and then is treated with $\mathrm{HCl}$.

(a). Effervesces.-Calcium oxalate. 
(b). Does not effervesce. The original powder is warmed with potassium hydrate solution.

1.-Ammonia is freely given off. The powder dissolves in acetic or hydrochloric acid, and this solution with ammonia gives a crystalline precipitate.-Triple phosphate.

2.-Ammonia is not given off, or but in traces. The powder dissolves as above and the solution with ammonia yields an amorphous precipitate.-Earthy phosphates.

B. It does burn.

(a). With a flame.

1.-The flame is yellow, lasting; odor of burnt feathers; insoluble in ether and alcohol.-Fibrin.

2.-The flame is yellow, lasting; odor of burning resin or shellac; soluble in ether and alcohol.-Urostealith.

3.-The flame is bluish; does not last; peculiar sharp odor; soluble in ammonia from which solution on spontaneous evaporation six-sided plates separate.-Cystin.

(b). Without a flame.

1.-Does not give the murexid test; dissolves in $\mathrm{HNO}_{3}$ without effervescence, and this solution on evaporation leaves a yellow residue which with alkalis turns orange, on heating becomes red.-Xanthin.

2.-Does give the murexid test. The original powder is treated with a little cold $\mathrm{KOH}$ solution.

(a). Ammonia is freely given off.-Ammonium urate.

(b). Ammonia is not given off, or but in traces.-Uric acid. 
Table of Atomic Weights.

(ACCORDING TO F. W. CLARKE.)

\begin{tabular}{|c|c|c|c|c|c|}
\hline Name. & Symbol. & $\begin{array}{l}\text { Atomic } \\
\text { weight. }\end{array}$ & Name. & Symbol. & $\begin{array}{l}\text { Atomic } \\
\text { weight. }\end{array}$ \\
\hline Aluminum. & $\mathrm{Al}$ & 27 & Molybdenum & Mo & 96 \\
\hline Antimony ..... & $\mathrm{Sb}$ & 120 & Nickel..... & $\mathrm{Ni}$ & 58.7 \\
\hline Arsenic ........ & As & 75 & Nitrogen... & $\mathrm{N}$ & 14.03 \\
\hline Barium ......... & $\mathrm{Ba}$ & 137 & Osmium .. & Os & 190.8 \\
\hline Bismuth ........ & $\mathrm{Bi}$ & 208.9 & Oxygen......... & $\mathrm{O}$ & 16 \\
\hline Boron........... & $\mathrm{B}$ & 11 & Palladium...... & $\mathrm{Pd}$ & 106.6 \\
\hline Bromin ......... & $\mathrm{Br}$ & 79.95 & Phosphorus..... & $\mathrm{P}$ & 31 \\
\hline Cadmium ... & $\mathrm{Cd}$ & 112 & Platinum ....... & $\mathrm{Pt}$ & 195 \\
\hline Cesium ......... & $\mathrm{Cs}$ & 132.9 & Potassium .... . . & $\mathrm{K}$ & 39.11 \\
\hline Calcium.... & $\mathrm{Ca}$ & 40 & Rhodium .... & Rh & 103 \\
\hline Carbon .... & $\mathrm{C}$ & 12 & Rubidium. & $\mathrm{Rb}$ & 85.5 \\
\hline Cerium.... & $\mathrm{Ce}$ & 140.2 & Ruthenium. & $\mathrm{Ru}$ & 101.6 \\
\hline Chlorin .... & $\mathrm{Cl}$ & 35.45 & Samarium. . & $\mathrm{Sm}$ & 150 \\
\hline Chromium. & $\mathrm{Cr}$ & 52.1 & Scandium . & $\mathrm{Sc}$ & 44 \\
\hline Cobalt...... & $\mathrm{Co}$ & 59 & Selenium... & Se & 79 \\
\hline Columbium. & $\mathrm{Cb}$ & 94 & Silicon .... . & $\mathrm{Si}$ & 28.4 \\
\hline Copper..... & $\mathrm{Cu}$ & 63.6 & Silver..... & $\mathrm{Ag}$ & .107 .92 \\
\hline Didymium... & $\mathrm{Di}$ & 142.3 & Sodium.... & $\mathrm{Na}$ & 23.05 \\
\hline Erbium.... & Er & 166.3 & Strontium. . & $\mathrm{Sr}$ & 87.6 \\
\hline Fluorin.... & $\mathrm{F}$ & 19 & Sulphur.... & $\mathrm{S}$ & 32.6 \\
\hline Gallium ....... & $\mathrm{Ga}$ & 69 & Tantalum.. & $\mathrm{Ta}$ & 182.6 \\
\hline Germanium . & $\mathrm{Ge}$ & 72.3 & Tellurium ... & $\mathrm{Te}$ & 125 \\
\hline Glucinum .. & $\mathrm{Gl}$ & 9 & Terbium ... & $\mathrm{Tb}$ & 159.5 \\
\hline Gold....... & $\mathrm{Au}$ & 197.3 & Thallium ... & $\mathrm{Tl}$ & 204.18 \\
\hline Hydrogen .. & $\mathrm{H}$ & 1.007 & Thorium.... & Th & 232.6 \\
\hline Indium .... & In & 113.7 & Tin......... & $\mathrm{Sn}$ & 119 \\
\hline Iodin... & $\mathrm{I}$ & 126.85 & Titanium .. & $\mathrm{Ti}$ & 48 \\
\hline Iridium ..... & $\operatorname{Ir}$ & 193.1 & Tungsten .... & W & 184 \\
\hline Irol & $\mathrm{Fe}$ & 56 & Uranium ... & $\mathrm{U}$ & 239.6 \\
\hline Lanthanum & La & 138.2 & Vanadium. . & $\mathrm{V}$ & 51.4 \\
\hline Lead .. & $\mathrm{Pb}$ & 206.95 & Ytterbium .. & $\mathrm{Yb}$ & 173 \\
\hline Lithium. & $\mathrm{Li}$ & 7.02 & Yttrium ... & $\bar{Y} t$ & 89.1 \\
\hline Magnesium & $\overline{\mathrm{Mg}}$ & 24.3 & Zinc........ & $\mathrm{Zn}$ & 65.3 \\
\hline Manganese. & Mn & 55 & Zirconium...... & $\mathrm{Zr}$ & 90.6 \\
\hline Mercury ... . & $\mathrm{Hg}$ & 200 & & & \\
\hline
\end{tabular}




\section{CHAPTER XI.}

\section{QUANTITATIVE ANALYSIS.}

Quantitative analysis can be carried out in two ways: gravimetrically or by weight, and volumetrically or by measure.

In gravimetric determinations the substance to be estimated is converted into an insoluble form which is then. removed by filtration, washed free of all impurities, dried and weighed. In some instances the precipitate is not weighed directly but is first ignited and thus converted into a more stable form. As an illustration we may mention the estimation of albumin in urine. This is converted into an insoluble form by the action of heat; the coagulated albumin is collected on a previously weighed filter, washed, dried and weighed. After deducting the weight of the dried filter, the difference represents the weight of albumin present in the quantity of urine taken for the examination. Usually substances cannot be weighed directly, as such, but must be combined with other metals or acids to form insoluble compounds. Thus, if it is desired to estimate the chlorine present in urine the most convenient procedure is to precipitate the chlorides present with silver nitrate. The silver chloride is then collected, washed, dried, ignited and weighed. From the amount of silver chloride found it is easy to calculate the corresponding amount of $\mathrm{Cl}$, or of $\mathrm{NaCl}$. Similarly, when sulphuric acid is estimated, advantage is taken of the fact that it forms an insoluble compound with barium chloride. The barium sulphate is collected on a filter, washed, dried, ignited and weighed, and from the weight obtained the amount of $\mathrm{SO}_{3}$ present can be calculated. 
The ignition serves to destroy the filter and in some cases also alters the composition of the precipitate. Thus, calcium oxalate on ignition is converted into calcium oxide; magnesium ammonium phosphate is changed to the pyrophosphate. The filter on incineration leaves a certain amount of ash, the weight of which should be known, as well as that of the crucible in which the ignition takes place; these weights must therefore be deducted from the final weight in order to obtain the weight of the precipitate.

When a precipitate is filtered off, the filtrate should come through perfectly clear. If it does not do so it should be returned to the filter until it does come through clear, otherwise a loss of the precipitate would result. Furthermore, great care must be taken to wash the precipitate on the filter till all the substances that may be present in solution are washed out of the filter and precipitate. If for instance chlorides are being estimated, the silver chloride is washed on the filter till a portion of the wash-water ceases to give the slightest cloud on addition of $\mathrm{HCl}$. This indicates that all the silver nitrate has been removed by the washing.

In volumetric analysis the amount of the substance present is ascertained by means of solutions of known strength. These are spoken of as standard and are of two kinds: empirical and normal.

An empirical solution is of such strength that one c.c. will react with exactly so many $\mathrm{mg}$. of the substance to be analyzed as may be desired. In other words one c.c. of an empirical solution indicates $1,5,10$, or any number of $\mathrm{mg}$. of that substance according to its strength. It may be made to represent any number of milligrams of such substance as may be convenient for purpose of calculation. Thus, we may make several empirical solutions of silver 
nitrate: one c.c. of one solution may represent $1 \mathrm{mg}$. of $\mathrm{Cl}$; that of another $10 \mathrm{mg}$. of $\mathrm{Cl}$; that of a third $10 \mathrm{mg}$. of $\mathrm{NaCl}$, etc.

To prepare an empirical solution write out first of all the equation representing the reaction between the reagent and the substance to be analyzed. Thus, we will prepare an empirical solution of $\mathrm{AgNO}_{3}$ such that one c.c. will exactly precipitate all the chlorine contained in $10 \mathrm{mg}$. of $\mathrm{NaCl}$. In other words one c.c. is to represent $10 \mathrm{mg}$. of $\mathrm{NaCl}$.

$$
\underset{170}{\mathrm{AgNO}_{3}}+\underset{58.5}{\mathrm{NaCl}}=\mathrm{AgCl}+\mathrm{NaNO}_{3}
$$

It is evident that one molecule of $\mathrm{AgNO}_{3}$ will react with one of $\mathrm{NaCl}$, and since their molecular weights are 170 and 58.5 respectively it follows that $170 \mathrm{~g}$. of silver nitrate will react with $58.5 \mathrm{~g}$. of sodium chloride. The question then is, how much $\mathrm{AgNO}_{3}$ will react with $.010 \mathrm{~g}$. of $\mathrm{NaCl}$ ? This is easily found by the following proportion:

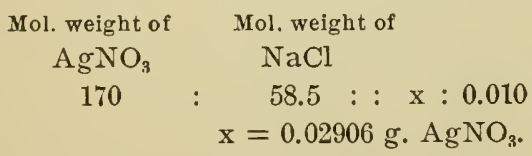

That amount of $\mathrm{AgNO}_{3}$ must be present in one c.c. in order that this shall react with $10 \mathrm{mg}$. of $\mathrm{NaCl}$. Therefore, $29.060 \mathrm{~g}$. of $\mathrm{AgNO}_{3}$ dissolved in water and diluted to one liter will give an empirical solution such that one c.c. represents $10 \mathrm{mg}$. of $\mathrm{NaCl}$.

Calculate the amount of reagent necessary to make a

\begin{tabular}{|c|c|c|c|c|c|}
\hline " & " & 66 & 66 & “6 & $2 \mathrm{mg}$. of $\mathrm{KCl}$. \\
\hline $\mathrm{BaCl}_{2}$ & 66 & "6 & 66 & 66 & $10 \mathrm{mg}$. of $\mathrm{SO}_{3}$; \\
\hline & 6 & 16 & "6 & " & $10 \mathrm{mg}$. of \\
\hline
\end{tabular}
liter of empirical solution of each of the following: 
How much $\mathrm{Hg}, \mathrm{HgO}, \mathrm{Hg}\left(\mathrm{NO}_{3}\right)_{2}$ must be dissolved and diluted to one liter in order to make an empirical solution such that one c.c. will represent $10 \mathrm{mg}$. of urea?

A normal solution is one that contains in one liter one gram of basic hydrogen or its equivalent. Thus, in $\mathrm{HCl}$ the hydrogen is basic; moreover one part of hydrogen unites with 35.5 parts of chlorine to make 36.5 parts of $\mathrm{HCl}$. In other words $1 \mathrm{~g}$. of basic hydrogen is contained in $36.5 \mathrm{~g}$. of $\mathrm{HCl}$. This amount of $\mathrm{HCl}$, dissolved and diluted to one liter, will give therefore a normal solution of $\mathrm{HCl}(\mathrm{N})$.

Again, $\mathrm{H}_{2} \mathrm{SO}_{4}=2+32+64=98$. That is, in $98 \mathrm{~g}$. of $\mathrm{H}_{2} \mathrm{SO}_{4}$ there are $2 \mathrm{~g}$. of basic hydrogen. The definition above calls for one $\mathrm{g}$. of basic hydrogen, hence $49 \mathrm{~g}$. of $\mathrm{H}_{2} \mathrm{SO}_{4}$ dissolved and diluted to one liter would give a normal $\mathrm{H}_{2} \mathrm{SO}_{4}$ solution.

Calculate the amount necessary to make one liter of normal solution of each of the following: $\mathrm{HNO}_{3}, \mathrm{H}_{3} \mathrm{PO}_{4}$, anhydrous oxalic acid $\mathrm{C}_{2} \mathrm{H}_{2} \mathrm{O}_{4}$, crystallized oxalic acid $\mathrm{C}_{2} \mathrm{H}_{2} \mathrm{O}_{4}+2 \mathrm{H}_{2} \mathrm{O}$, acetic acid $\mathrm{C}_{2} \mathrm{H}_{4} \mathrm{O}_{2}$. In calculating these bear in mind that a normal solution does not call for $1 \mathrm{~g}$. of hydrogen but for $1 \mathrm{~g}$. of basic hydrogen.

The calculation for a normal alkali solution, such as sodium hydrate, is as follows:

$$
\mathrm{NaOH}+\mathrm{HCl}=\mathrm{NaCl}+\mathrm{H}_{2} \mathrm{O} \text {. }
$$

$40 \quad 36.5$

$40 \mathrm{~g}$. of sodium hydrate, then, combine with $36.5 \mathrm{~g}$. of hydrochloric acid containing one gram of basic hydrogen. Therefore $40 \mathrm{~g}$. $\mathrm{NaOH}$ contain the equivalent of one gram of basic hydrogen, and when dissolved and diluted to one liter make a normal solution.

Calculate how much sodium carbonate $\left(\mathrm{Na}_{2} \mathrm{CO}_{3}\right)$ is contained in a normal solution. Also calculate the amounts 
of $\mathrm{KOH}, \mathrm{Ba}(\mathrm{OH})_{2}, \mathrm{Ag}_{2} \mathrm{O}$ respectively that must be taken and dissolved to make a liter of normal solution.

The normal solutions are too strong for most purposes, hence solutions of fractional strength are employed. Thus, a semi-normal solution of hydrochloric acid, $\frac{N}{2} \mathrm{HCl}$, is one which contains $18.25 \mathrm{~g}$. $\mathrm{HCl}$ per liter. A deci- or tenthnormal solution $\left(\frac{\mathrm{N}}{\mathrm{r}}\right)$ contains one-tenth and a centi-normal solution ( mal one does.

As a rule deci-normal solutions are most convenient and are therefore employed most often.

One c.c. of any normal acid solution will exactly neutralize 1 c.c. of any normal alkali solution. This, follows from the definition of such solution. Thus, $36.5 \mathrm{~g}$. of $\mathrm{HCl}$ neutralize $40 \mathrm{~g}$. of $\mathrm{NaOH}$. The one contains one gram of basic hydrogen, the other its equivalent. Since these amounts are each contained in one liter of corresponding normal solutions, it follows that a liter of one will neutralize a liter of the other; or one c.c. of one will neutralize one c.c. of the other.

Furthermore, 1 c.c. of a normal solution corresponds to 10 c.c. of a deci-normal solution; 1 c.c. of a deci-normal solution corresponds to 1 c.c. of any other deci-normal solution. The same is true of other fractional normal solutions.

The word factor means the amount of the reagent contained in 1 c.c. of the solution. Thus, the $\mathrm{N}$ factor of $\mathrm{NaOH}$ is 0.040 ; the $\frac{\mathrm{N}}{10}$ factor of hydrochloric acid is 0.00365 . When speaking of empirical solutions the word factor means the amount of the substance to be analyzed represented by one c.c. of that solution.

The term titration is employed to denote the method of estimating a given substance in solution by means of a standard solution. Thus, we can estimate the amount of 
chlorides in urine by titration with a standard solution of silver nitrate.

Indicators.-For the purpose of determining when a reaction is completed an indicator is, as a rule, necessary. An indicator usually shows by a change in the color of the solution when enough of the standard solution has been added. Thus, in titrating an alkaline solution, with litmus as an indicator, the moment a red color appears we know that the reaction has changed from alkaline to acid, and that enough of the standard acid solution has been added.

In preparing standard solutions of such reagents as do not change when exposed to air, the required amount can be weighed directly, then dissolved and diluted to one liter. This is true of such substances as silver nitrate, copper sulphate, oxalic acid, etc.,--substances which are crystalline and neither absorb moisture nor give off water of crystallization when exposed. But the case is different with such substances as $\mathrm{NaOH}$ which rapidly absorb moisture and carbon dioxide, or with the common acids which have a variable strength. In such cases, it is customary to dissolve a little more than the required amount of the reagent, then determine the strength of this solution gravimetrically, or volumetrically. The preparation of $\frac{\pi}{10} \mathrm{NaOH}$ as given on p. 239 will illustrate this point.

When a substance contains water of crystallization this must be taken into account in the calculation since it is for the time being a part of the molecule, and although the water plays no part in the reaction it nevertheless weighs something.

A good balance is necessary for quantitative analysis. Certain rules regarding the use of the balance should be closely adhered to. The first thing to do is to see that the scales balance, that is, that the pointer swings the same number of divisions on each side of the zero point. The 
first, or starting swing is disregarded, the second and third are, however, noted. If the scales do not balance they should be made to do so, either by cleaning the watchglasses and pans, or by turning the adjustment.

The substance is weighed either on the watch-glass or in a weighing-bottle, never on the pan direct. The substance should always be placed on the left pan; the weights on the right pan. Whenever a portion of the substance, or a weight is to be placed on, or taken off the balance, the latter should first be brought to a perfect rest.

The weights should never be handled with the fingers, but should always be picked up with a pair of clean forceps. When weighing the weights should not be placed on the pan at random, guessing that this or that much will be enough, but they should be placed on in regular order. Thus, for example, in weighing a crucible it is found that $10 \mathrm{~g}$. is too much. This is taken off and $5 \mathrm{~g}$. placed on; as this is too light the $2 \mathrm{~g}$. piece is added; this also is insufficient and so a $1 \mathrm{~g}$. piece is added. This is still not enough so another $1 \mathrm{~g}$. weight is placed on the pan. The total weight of $9 \mathrm{~g}$. is still less than that of the crucible, which, however, does not weigh $10 \mathrm{~g}$. The $500 \mathrm{mg}$. piece is then placed on the pan, but the total weight is now too heavy; the crucible weighs less than $9.5 \mathrm{~g}$. The $200 \mathrm{mg}$. piece is substituted but this is likewise too heavy; then a $100 \mathrm{mg}$. piece is tried. This is too light; the weight of the crucible lies between 9.1 and 9.2. Now the $50 \mathrm{mg}$. piece is added; this is not heavy enough, and so the $20 \mathrm{mg}$. piece is placed on; this is still too light and a $10 \mathrm{mg}$. piece is added; as this is not enough the second $10 \mathrm{mg}$. piece is added; but even now the weight is low. It lies, therefore, between 9.190 and 9.2. The rider is now placed at 5 , or in its absence the $5 \mathrm{mg}$. piece is added. If the $5 \mathrm{mg}$. piece is too heavy proceed exactly as above with the $500 \mathrm{mg}$. piece; if it is not heavy enough proceed as with the $50 \mathrm{mg}$. piece. When weighing with less than the $10 \mathrm{mg}$. piece, or when using the rider, 
the door of the balance should be closed. The balance should be sensitive to one milligram and a good one should read to $\frac{1}{10} \mathrm{mg}$.

To measure out liquids the graduated cylinder, pipette, or burette is made use of. The cylinder is not very accurate and should not be used except to measure out roughly 10, 25, 50 c.c., etc. Each student is provided with a pipette and a burette, holding 10 and 50 c.c. respectively, and graduated in $\frac{1}{10}$ c.c. These are to be used for measuring out solutions for exact work. The surface of the liquid in the pipette or burette is concave, forming the so-called meniscus. When taking a reading the eye should be placed on a level with the lower border of the meniscus since this is more distinct than the upper.

If the measuring instrument is not perfectly dry it should be washed with distilled water, then rinsed two or three times with small portions of the liquid that is to be measured. The outside of the pipette or burette should be wiped dry. When using the burette care must be taken to expel any air that may remain in the tip. If the pipette or burette is dirty, that is, if water does not flow readily and smoothly but adheres in drops, here and there, it can be cleaned by filling with the chromic acid cleaning mixture and allowing it to stand thus over night.

In gravimetric work the amount of ash given by the filter paper on ignition should be known. It is best to use filters washed in $\mathrm{HCl}$ and $\mathrm{HF}$. The ash from these is so small that it may be disregarded. The paper should be folded carefully, and unless a dry filter is called for, it should be moistened. The tip of the funnel should not be so far above the surface of the filtrate in the beaker as to cause spattering. The filter should never be filled too full, since many precipitates tend to creep up and a portion may thus be lost. A glass rod covered with a short piece of 
rubber tubing, $1 / 2$ inch long, is of great use in transferring precipitates from the beaker or flask to the filter.

The filtrate should always be received in a clean beaker or flask, and the first portion should be tested to ascertain if the precipitation is complete by adding some of the reagent used. In washing, small amounts of wash-water should be used, and each portion should be allowed to run through before another is added. If the liquid does not filter clear, the filtrate should be returned to the filter a second, or even third time if necessary.

The funnel with the filter containing the precipitate is placed in an air-bath at $100^{\circ}$ to dry. It should be covered with paper.

Everything that is set aside should be properly labelled, and covered to protect it from dust. The weighings and other results should be recorded at once in a note-book, and not kept on scraps of paper, or trusted to memory.

\section{Quantity of Urine.}

The quantity of urine passed during a period of twenty-four hours is of great importance both as an indication of the presence or absence of certain diseases, as well as the basis of computations in the quantitative analysis of this secretion. During a period of twenty-four hours there are hourly variations in the volume of urine and in the amount of solids present, and consequently analytical results can have but little value unless obtained from a fair sample of the entire day's urine.

In collecting the urine care should be taken to empty the bladder before entering upon and again at the close of the 24 hour period. Furthermore, loss of urine at stool should be avoided. Urine should always be received in perfectly clean vessels, care being taken to avoid the accidental introduction of fats, oils, starches, fibers and the 
like. The urine may be measured in ounces, pints or quarts, but it is much better to procure a metric graduate expressing the volume in so many cubic centimeters. It may be well to bear in mind that a quart is a little less than a liter.

An adult man excretes on an average about 1500 c.c. of urine per day; women excrete less, about 1200 c.c. This difference in volume corresponds to a like difference in the amounts of urea, uric acid, etc., excreted, and is due to the different conditions under which the two sexes live rather than to any difference of sex proper. The average volume of the urine of 333 male students at Ann Arbor was 1120 c.c., and the average specific gravity for the same number was 1.022. The average volume of urine of 56 female students was 1000 c.c., and the average specific gravity was 1.020. Infants excrete, relatively, 3-4 times as much urine as adults. Children, likewise, excrete relatively more, whereas in old age the excretion is less.

Great variations in the quantity of urine may be expected in different individuals and even in the same individual, depending on certain conditions mentioned below. Regardless of the variation in the volume of the urine of a healthy person it can be said that the solids in solution remain constant, whether the volume is great or small. In diseased conditions, on the other hand, variations in volume and in solids will be met with. The least secretion of urine takes place during the night, toward morning; whereas greatest secretion is observed after rising and especially an hour or two after meals.

When the volume of urine is materially increased this condition is designated as polyuria. A diminished secretion. of urine is known as oliguria, and suppression of urine secretion is expressed by the term anuria.

There are certain factors which markedly influence the volume of urine in health and in disease. These may be briefly touched upon. 
1.- The amount of water ingested; whether taken as such, or as milk, coffee, tea, beer and the like, it promptly increases the volume of urine. It should, moreover, be remembered that animal and vegetable food contains nearly three-quarters of its weight of water. This diuretic action of water can be readily utilized whenever it is necessary or desirable to flush out the kidneys.

2.-The kind and amount of food is to be considered. As just indicated a large percentage of ordinary food is water. The percentage is still greater ( 87 per cent) in the case of milk. Moreover, the hydrogen in the starch, fat, or proteid molecule is largely oxidized in the body to water, and hence it is that more urine may be excreted than the amount of water actually consumed. Furthermore, in starvation, urine continues to be excreted even when no water is taken. This is due to the setting free of water held by the tissues when they are broken down, and to the formation of water by the oxidation of the hydrogen of such disintegrated tissue. In general, the tissue thus broken down will yield about three-fourths of its weight of water. Certain constituents of food by their diuretic action increase the volume of urine. This is true of salt, onions, coffee, tea, etc.

3.--The amount of water that leaves the body otherwise than by the kidneys. These other channels of elimination are the skin, lungs, stomach and intestines. In addition to these water may be diverted from the kidneys to form exudates or transsudates, as in odema and in ascites. A warm temperature and much exercise favor the loss of water by perspiration and by exhalation. Thus, after much walking or wheeling in summer time the amount of urine is small (several hundred c.c.) although an enormous amount of water may be drunk. Such urine has a high specific gravity and is highly colored. Prolonged vomiting may serve to remove water and with this, injurious waste products. In intestinal diseases, especially where a diarrhoic 
condition exists as in cholera, enormous loss of water may occur through the discharges and as a result the quantity of urine will be diminished and even complete anuria may result.

4.-Nervous influences may affect the volume of urine, as in hysteria; possibly also in diabetes insipidus.

5.-Diseases of the kidney, heart, lungs, etc.

An increased excretion of urine is met with in diabetes insipidus, in which condition the volume of urine may be several times that of the normal, and inasmuch as there is no increase in solids the specific gravity may be very low and the color very light.

In diabetes mellitus, contrary to other diseases or conditions, the specific gravity of the urine (1.030-1.040 or more) increases with the increase in the volume of the urine. A diabetic person on an average eliminates 5 to 6 liters of urine per day, but it is not unusual to find double this amount. As a rule the more water that is excreted, the more sugar there is present in the urine and hence the increase in specific gravity. This great increase of urine in a diabetic person may be counteracted by a febrile disease, in which case the volume may be diminished, though not always, to less than the normal volume. The urine is increased in icterus after the obstruction in the bile duct has been removed.

A decrease in the volume of urine is of considerable importance and is met with in various diseases. In dyspnoic conditions, due to disturbances of the circulatory or respiratory apparatus, the urine is diminished in volume; the specific gravity is increased and the color is deepened; it is strongly acid in reaction, and urate sediments are common. This is due to loss of water by the lungs, skin, and by transsudates.

In febrile diseases, until crisis occurs, the volume of the 
urine is small, the specific gravity is high and the urine possesses the characteristics just mentioned. In a person having contracted kidneys, or affected with diabetes, the volume of the urine is not diminished, as a rule, below that of normal urine. When crisis occurs, as in pneumonia, scarlet fever, etc., there is at once a marked increase in volume, the specific gravity drops and the color becomes. lighter. In recovery from protracted fever, as typhoid, the urine gradually returns to normal volume, specific gravity and color.

In rheumatism the urine is small in amount, with high specific gravity and a sediment as in fevers. Toward the end of the attack the urine is increased in amount and the specific gravity decreases. In starvation, with or without water being taken, there is a decrease in the volume of urine. A fasting person does not drink much water, and moreover the loss of water by the lungs and skin continues.

In diseases of the liver the amount of urine varies considerably, depending on the amount of water ingested and whether or not ascites exists. In stomach diseases the volume may be greatly diminished, depending on the amount of food taken, and the extent of absorption. The latter may be diminished by vomiting or otherwise, and hence the urine will be small in volume. In kidney diseases the volume of the urine may vary from complete anuria to marked polyuria.

\section{I.-Determination of the Quantity of Urine per 24 Hours.}

(a). By rolume.-Cylinders graduated in cubic centimeters should be employed.

(b). By weight.-This is more accurate but is less commonly used. 


\section{Specific Gravity of Urine.}

The specific gravity of urine depends upon the volume and on the amount of solids held in solution. The density of normal urine consequently varies considerably, but as a rule it lies between 1.017 and 1.020. As a result of drinking much water it may fall to 1.010 or 1.005 , whereas when very little water is taken it may rise to 1.030 . As stated above, in a healthy person the total amount of solids excreted is quite constant, from day to day, regardless of the quantity of the urine. This great variation in the specific gravity of normal urine depends almost wholly on the amount of water taken into the body and excreted by the kidneys. If considerable water is lost by perspiration or by exhalation, as after much exercise in warm weather, the volume of the urine, as already pointed out, will be greatly decreased and, as there is no difference in the excretion of solids, it follows that the specific gravity will be increased. The solids which especially affect the specific gravity, since they make up two-thirds of the total amount present, are urea and sodium chloride.

The determination of the specific gravity of urine is one of the most important steps taken in the analysis of urine. It gives valuable information as to the extent of tissue metabolism and the efficiency of the kidneys. It is necessary, however, to always bear in mind not only the amount of water ingested but also the amount of water that leaves the body by other channels than the kidneys, such as by perspiration, exhalation, exudation, vomiting and diarrhoea. Likewise the kind and amount of food taken must be considered.

A high specific gravity and a small amount of urine are met with especially in acute febrile diseases. Although such persons take little or no food, the amount of urea, as 
has been pointed out (p. 129), is considerably increased. Furthermore, the amount of water taken in is small and a considerable amount is lost through other channels than the kidneys. A high specific gravity, therefore, indicates increased tissue destruction. In convalescence there is less tissue destruction, hence less urea is formed, and this with the increased quantity of urine gives a low specific gravity.

A high specific gravity and a small amount of urine are met with in many mental disorders, such as delirium, and in all diseases which lead to venous stasis. This is due to the decrease in the elimination of water. Furthermore, the very highest specific gravity of urine (1.040-1.050) is met with frequently in diseases of the kidney, especially in the later stages. This is due to the presence of albumin and to the retention of water. In the early stages of kidney diseases, however, the urine is considerably increased in quantity and although albumin is present, the specific gravity is low.

A low specific gravity is met with in all conditions which increase the quantity of the urine, except in diabetes mellitus. Such conditions are convalescence, early stages of kidney diseases, and diabetes insipidus (1.010 or less).

A low specific gravity and a small amount of urine are observed in many chronic diseases, and just before death from acute diseases. It indicates slow metabolism, or retention and points to danger from uræmia.

A high specific gravity and a large volume of urine are met with in only one disease, diabetes mellitus. The increased density, in spite of the enormous amount of water passed, is due to the large amounts of sugar, urea, etc., which are excreted. This condition of the urine is therefore a strong indication of the presence in the urine of sugar 
and the latter should at once be tested for. On the other hand, a low specific gravity and a large amount of urine should lead to the suspicion of albuminuria, and tests for albumin should be made.

\section{Determination of the Specific Gravity.}

(a). With the urinometer.-The spindle of this instrument is graduated from 1.000 to 1.040 at a temperature of $15^{\circ} \mathrm{C}$. Hence, if the temperature is higher, as is usually the case, the observed reading should be reduced to the normal temperature of $15^{\circ}$.

Pour the urine to be tested into a cylinder which should be wide enough to allow the urinometer free motion. Avoid the formation of foam and if any results remove it by means of a piece of filter paper. Immerse the clean dry urinometer and after it comes to rest read off the point where the lower border of the meniscus cuts the scale. To make sure that the instrument floats freely gently touch the stem of the urinometer and after it comes to rest take a second reading. The normal specific gravity varies from 1.017-1.020. (See p. 230).

To reduce the observed reading to the normal temperature, take the temperature of the urine and for every $3^{\circ}$ above $15^{\circ}$ add one division, and for every $3^{\circ}$ below subtract one division from the observed reading. Thus:

The specific gravity of a urine is 1.017 and the temperature is $24^{\circ} .24-15=9$. Therefore, $1.017+3=1.020$, the correct reading.

If a sediment of urates is present in the urine this should be gently warmed till they are dissolved. Otherwise it is desirable, though not necessary, to filter the urine. In order to draw accurate conclusions, the specific gravity should not be taken of a portion of the urine passed at one time but of the mixed 24 hours' urine.

The urinometer offers the quickest means for taking the density of a urine. The results obtained are very satisfactory, especially if the correction is made for temperature as indicated above, and if a set of two or of four urinometers is used. The ordinary urinometer is graduated from 1.000 to 1.040 and consequently each degree is 
so small that an error in reading may easily occur. This error can be obviated by using a set of two urinometers, one of which is graduated from 1.000 to 1.020 ; the other from 1.020 to 1.040 . Better still is a set of four urinometers graduated as follows: from 1.000 to $1.010 ; 1.010$ to 1.020 ; 1.020 to 1.030 ; and 1.030 to 1.040 . With the latter set it is possible to read to the fourth decimal place. Whereas, on the ordinary urinometer the 40 divisions are crowded into about $3 \mathrm{~cm}$., in the set mentioned above they extend over about $20 \mathrm{~cm}$., hence the greater accuracy in reading.

Reduction of the observed specific gravity to that corresponding to the normal volume of 1500 c.c.: multiply the quantity of urine per 24 hours by the decimal portion of the specific gravity, divide by 1500 and add the result to 1 . Thus, the volume of a urine is 1820 c.c., and the specific gravity is 1.014 .

$$
1 .+\frac{1820 \times .014}{1500}=1.017 \text {. }
$$

Again, the volume is 4500 c.c., and the specific gravity is 1.030 . Therefore:

$$
\text { 1. }+\frac{4500 \times .030}{1500 .}=1.090 \text {. }
$$

(b). With the picnometer, or specific gravity bottle. This is the most accurate means for ascertaining the specific gravity but it is employed only for very exact work.

\section{Determination of Total Solids.}

(a). Neubauer's aproximate nethod.-Multiply the last two figures of the specific gravity by 2.33: this gives the weight of solids in 1000 c.c. To obtain the solids in the $2 t$ hours' urine multiply this result by the number of liters of urine.

Examples. - The volume of the urine is 1820 c.c. and the specific gravity is $1.014 .14 \times 2.33 \times 1.820=59.37 \mathrm{~g}$. of solids in the 24 hours' urine. 
Again, the volume is 4500 c.c. and the specific gravity is 1.030 . $30 \times 2.33 \times 4.500=314.55 \mathrm{~g}$. of solids per 24 hours' urine.

(b). Direct determination. - This is rarely resorted to except for scientific purposes. Inasmuch as a part of the urea is decomposed, during the process of evaporation, into ammonia and carbonic acid, the amount of ammonia given off must be determined, and the result converted into urea. The amount of urea thus ascertained to be broken up by the evaporation of a known volume of the urine on the water-bath is added to the weight of the residue obtained.

The amount of total solids present in normal urine is quite constant, regardless of variation in the volume of the urine. The average amount of solids is about $60 \mathrm{~g}$. The organic compounds make up about $35 \mathrm{~g}$. and the inorganic compounds about $25 \mathrm{~g}$. Urea is the chief organic compound and amounts to about $30 \mathrm{~g}$. Common salt is the chief inorganic constituent and makes up about $15 \mathrm{~g}$. For the quantities of other constituents present see the table at the end of this chapter.

The urinometer gives the specific gravity of the urine, and from this the amount of total solids can be estimated. In a somewhat similar manner the amount of urea, of sugar and of albumin may be estimated from the readings of the urinometer.

\section{Reaction of Urine.}

The reaction of normal urine is usually acid, due to the presence of the acid phosphate of potassium or sodium, $\mathrm{NaH}_{2} \mathrm{PO}_{4}$. The presence of $\mathrm{Na}_{2} \mathrm{HPO}_{4}$ decreases the acidity and may even render the urine neutral or amphoteric in reaction. It has always been an interesting question as to how an acid urine is secreted from an alkaline blood. The reaction of blood, as seen from previous experiments, is 
strongly alkaline, due to alkaline carbonates and phosphates. It has been supposed that the absorption of the free hydrochloric acid from the stomach and the formation of sulphuric and phosphoric acids by the oxidation in the body of sulphur and phosphorus containing substances, led to the formation of acid phosphates in the blood. These acid salts, as is well known, can dialyze more readily than alkaline phosphates through an animal membrane, and hence would appear in the urine and by their preponderance impart the characteristic reaction to the secretion (Maly). According to Liebermann the formation of acid phosphates takes place in the renal epithelial cells. These were found to contain a compound of lecithin and albumin, lecithalbumin, which possessed marked acid properties. The alkaline urates and phosphates, consequently, come in contact with this lecith-albumin which takes up an atom of sodium from each and leaves acid urates and acid phosphates which then pass out into the urine.

A similar instance of mass action is seen in the change in reaction that frequently takes place in urine on standing. At the time of passage it may be intensely acid, but on cooling and standing it becomes less acid, and even neutral, and free uric acid appears in the sediment. By virtue of mass action, assisted by the low temperature, the acid phosphates remove the alkali from the uric acid and thus set this compound free. At the same time they form neutral phosphates and hence the change in the reaction of the urine. When the temperature is raised the uric acid can regain the lost atom of sodium and hence passes into solution as an urate and at the same time the original acid reaction returns.

An acid fermentation of urine may take place and thus the acidity may be considerably increased. 'Thus, in hydrogen sulphide fermentation the reaction will be intensely acid (p. 196). Acrain, if sugrar is present this may undergo 
lactic or butyric acid fermentation. The cause of such acid fermentations is invariably one or more species of bacteria.

The urine may be alkaline due either to sodium or to potassium carbonate (fixed alkali), or to ammonia or ammonium carbonate (volatile alkali) (p. 136). When due to fixed alkali the cause is invariably to be found in the food. As pointed out under urea (p. 126) the salts of most organic acids are oxidized to the corresponding carbonates. Thus, potassium acetate, citrate, malate, etc., are each converted into potassium carbonate and this is then excreted by the urine. It is possible, therefore, by direct administration of such salts to convert a highly acid urine into one having less acidity, or even an alkaline reaction. On the other hand many articles of food, as fruits and potatoes, contain such organic salts and hence impart an alkaline reaction to the urine.

An alkaline reaction due to volatile alkali is practically met with only in ammoniacal fermentation. All urine, sooner or later, undergoes ammoniacal fermentation, the urea becoming hydrated to ammonia and carbonic acid. However, it is only when this change occurs in the bladder, when the urine at the time of passage has an ammoniacal reaction, that it possesses a pathological significance. The cause of ammoniacal fermentation is always a micro-organism introduced in some way from without the body (see p. 137).

While the reaction of the mixed, twenty-four hours' normal urine is usually acid, this is not the case with the several portions passed during that period. There may be hourly variations in the reaction as well as in the quantity and composition of the urine secreted. Thus, the urine secreted during digestion (3-6 hours after meals) may be neutral, or even temporarily alkaline, due to the secretion of a large amount of hydrochloric acid into the stomach. 
The more hydrochloric acid secreted into the gastric juice the more marked will be the decrease in the acidity of the urine. In diseases of the stomach, such as cancer, where little or no acid is secreted it follows that there will be no effect observed on the acidity of the urine. The morning urine is often neutral or alkaline in reaction, whereas that secreted in the afternoon or early evening is the most acid.

From what has been said it is evident that the food is of first importance as affecting the reaction of the urine. This is seen in the reaction of the urine of carnivorous as compared with that of herbivorous animals. The former is strongly acid, the latter neutral or alkaline. In the former case the protein matter of the food, rich in sulphur and phosphorus, is oxidized in the body and yields sulphuric and phosphoric acids. The food of herbivorous animals on the other hand is rich in organic salts, which are oxidized in the body to carbonates. It does not follow that all vegetable food will impart an alkaline reaction. If such food is rich in proteins, as in the case of cereals and legumens, it will yield almost as acid a urine as a meat food. Potatoes, fruit and the like are poor in proteins and relatively rich in organic salts, hence they impart an alkaline reaction. In starvation the urine is acid, owing to the fact that the animal is living on the proteins of its own tissues.

frhe influence of digestion on the reaction of urine has been indicated above. Muscular exercise is said to increase, and profude perspiration is said to decrease the acidity. The absorption of alkaline transudates may render the urine alkaline.

While it is true that an exclusively meat diet produces a strongly acid urine due to the sulphuric and phosphoric acids formed on oxidation, it does not follow that the administration of these acids will enable one to increase the acidity of the urine without limit. When the alkalis of the blood cannot be spared or are not sufficient in amount to 
combine with the acid administered the latter unites with the ammonia which normally would go to make urea, and appears in the urine as an ammonium salt. This actually takes place in the urine of man and of carnivorous animals. In herbivorous animals, however, it would seem as if the ammonia formed in protein disintegration was not at the ready disposal of the administered mineral acids and hence cannot neutralize and render harmless such acids. The continued administration of mineral acids in small doses to herbivorous animals results in the withdrawal or in the decrease of the alkaline carbonates of the blood to such an extent as to cause death.

The reaction of the urine, and bence diet, has special significance in connection with the formation of uric acid sediments and calculi. Food rich in proteins and poor in the bases necessary to combine with the sulphuric and phosphoric acids formed on oxidation should be avoided. Cheese, salted meat and fish are of this class and when used as a common article of food stones are frequent. The deposition of urate sediments can be largely controlled by the use of such food as fruits, potatoes, etc., which decrease the acidity of urine and in themselves do not furnish uric acid. The use of lithia waters, piperazin or lysidin regardless of the kind of food, can impart but little benefit.

Ammoniacal urine, because of the crystalline triple phosphate that deposits, is much more likely to cause the formation of calculi than a fixed alkaline urine. The fine, amorphous phosphates formed in the latter instance are easily washed out. Moreover, this reaction is usually temporary and is due to the food and can therefore be readily corrected. 


\section{Determination of the Acidity (or Alkalinity) of the Urine.}

Reagents. $-\frac{N}{10} \mathrm{NaOH}$ solution. This contains $4 \mathrm{~g}$. of $\mathrm{NaOH}$ in one liter; therefore each c.c. $=0.004 \mathrm{~g}$. of $\mathrm{NaOH}$. Inasmuch as $\mathrm{NaOH}$ readily absorbs $\mathrm{CO}_{2}$ and water from the air, it cannot be weighed out accurately enough for this purpose. Hence, weigh out about $3 \mathrm{~g}$. and dissolve in about 500 c.c. of water. This solution is now too strong and its strength must therefore be ascertained; the solution can then be diluted to the proper point.

${ }_{10}^{\mathrm{N}}$ Oxalic acid solution. -This contains $6.3 \mathrm{~g}$. of oxalic acid $\left(\mathrm{H}_{2} \mathrm{C}_{2} \mathrm{O}_{4}\right.$ $+2 \mathrm{H}_{2} \mathrm{O}$ ) in one liter; hence each c.c. $=0.0063 \mathrm{~g}$. of oxalic acid. Oxalic acid is not altered on exposure to the air and can therefore be weighed out directly.

Preliminury exercise in titration.-Place 10 c.c. of the oxalic acid solution in a small beaker, dilute with about 50 c.c. of water, add a few drops of phenol-phthaleïn solution to serve as indicator, and then add from a burette, drop by drop, stirring after each addition with a glass rod, the sodium hydrate solution until a faint but permanent pink color remains. The difference in the reading of the burette before and at the close of the titration gives the amount of sodium hydrate solution employed to neutralize 10 c.c. of $\frac{N}{10}$ oxalic acid.

Read off the volume of $\mathrm{NaOH}$ solution remaining in the cylinder and calculate the amount of water that must be added to make this strictly deci-normal. Thus, suppose that the 10 c.c. of $\frac{N}{10}$ oxalic acid required 9.2 c.c. of the $\mathrm{NaOH}$ solution for neutralization, and that 440 c.c. of $\mathrm{NaOH}$ solution remain in the cylinder:

$$
9.2: 10:: 440: x . \quad x=478 \text { c.c. }
$$

the volume to which the $\mathrm{NaOH}$ should be diluted in order that it shall be deci-normal.

Now repeat the titration with the properly diluted $\mathrm{NaOH}$ solution. If correct, 10 c.c. of the one should neutralize 10 c.c. of the other.

Applicution to the urine.-Owing to the color of the urine and the presence of certain organic substances phenol-phthalein cannot, unfortunately, be used and in its stead the less sensitive litmus papers are employed. Mix well the 24 hours' urine and if any urates are 
present dissolve them by the aid of gentle heat; measure out 100 c.c. into a beaker and from a burette run in $\frac{\mathrm{N}}{10} \mathrm{NaOH}, 1 / 2 \cdot \mathrm{c} . \mathrm{c}$. at a time, stirring after each addition. After each addition test the reaction with litmus papers and note the point where the reaction changes from acid to neutral or slightly alkaline. The latter will more likely be the case. Suppose that on the addition of 3 c.c. the reaction was still acid but when 3.5 c.c. were added it became slightly alkaline. Therefore the neutral point lies between 3 and 3.5 c.c.

To determine exactly the point of neutralization, measure out a fresh portion of urine, as above, and ada 3 c.c. of $\frac{\mathrm{N}}{10} \mathrm{NaOH}$. Then run in this reagent, drop by drop, stiring after each addition, and also testing the reaction after each addition with litmus papers till the neutral point is reached. When this is reached note the amount of ${ }_{10}^{\mathrm{N}} \mathrm{NaOH}$ used. Ascertain by calculation the amount necessary to neutralize the total 24 hours' urine.

Example.-Suppose 100 c.c. of the urine requires 3.4 c.c. $\frac{\mathrm{N}}{10} \mathrm{NaOH}$ and the 24 hours' urine amounts to 1250 c.c.

$$
100: 3.4:: 1250: x . \quad x=42.5 \text { c.c. } \frac{\mathrm{N}}{10} \mathrm{NaOH} .
$$

Results can be expressed in this way but it is better to convert the number of c.c. of $\frac{\mathrm{N}}{10} \mathrm{NaOH}$ into the corresponding amount of oxalic acid. Each c.c. of $\frac{\mathrm{N}}{10} \mathrm{NaOH}=0.0063 \mathrm{~g}$. of oxalic acid. Therefore, $42.5 \times 0.0063=0.26775 \mathrm{~g}$. of oxalic acid. 'That is to say, the acidity of the 24 hours' urine corresponds to that much oxalic acid.

If the urine is alkaline the degree of alkalinity can be ascertained by titrating with $\frac{\mathrm{N}}{10}$ oxalic acid. Or, a known amount of $\frac{\mathrm{s}}{10}$ oxalic acid, sufficient to produce an acid reaction, may be added and the liquid then titrated back to the neutral point with $\frac{\mathrm{N}}{10} \mathrm{NaOH}$. The difference between the amount of oxalic acid added and that left uncombined corresponds to the alkalinity of the urine. The result is converted into the corresponding amount of sodium hydrate. The results obtained by this method are not very exact, owing to the presence toward the end of the reaction of alkaline as well as acid phosphates and hence an amphoteric reaction may be manifest. 


\section{Determination of Chlorides.}

(a). VOLHARD'S METHOD.

Reugents:-

1.-Silver nitiate solution.-Dissolve $29.060 \mathrm{~g}$. of fused silver nitrate in one liter of water. Each c.c. $=10 \mathrm{mg}$. of sodium chloride.

2.-A cold saturated solution of ferric alum, or a 5 per cent. solution of ferric sulphate. This solution must be free from chlorides. It is used as an indicator.

3.-Potassium sulphocyanate solution.-Dissolve about $10 \mathrm{~g}$. of the salt in a $1 / 2$ liter of water and standardize the solution against the silver nitrate. This is done as follows: To 10 c.c. of the silver nitrate in a small beaker, diluted to about 50 c.c., add a few drops of the ferric solution and then nitric acid, drop by drop, till the mixture is colorless. Now run in from a burette the sulphocyanate solution, stirring well after each addition, till a permanent red color is obtained. Note the amount used. 10 c.c. of one should equal 10 c.c. of the other. If the sulphocyanate is too strong it must be diluted so that the two solutions have the same strength.

Excmple. -450 c.c. of the sulphocyanate solution is left in the cylinder; 10 c.c. of the silver nitrate solution require 8.2 c.c. of the former. $10-8.2=1.8$. That is, to every 8.2 c.c. of the sulphocyanate 1.8 c.c. water must be added.

$$
\text { Therefore, } 8.2: 10:: 450: x=548.8 \text {. }
$$

The 450 c.c. in the cylinder diluted to 548.8 c.c. give a solution of the proper strength.

Execution.-In a flask provided with a 100 c.c. mark place 10 c.c. of urine, $20-30$ drops of dilute nitric acid (1.185 sp. gravity) then several drops of the ferric solution. Now run in from a burette the silver solution till the precipitate ceases to form. Add a few c.c. more of the reagent. Note the total amount used. Fill up to the 100 c.c. mark with water, mix and filter through a dry filter. To 50 c.c. of the filtrate run in from a burette the sulphocyanate solution, stirring well, till a faint but permanent red tinge results. Note the amount of sulphocyanate used, multiply it by two and deduct this from the amount of silver nitrate used. The difference is the amount 
of silver nitrate solution which combined with the chlorine. Now calculate the amount of $\mathrm{NaCl}$ in the 24 hours' urine.

Example.-The 24 hours' urine amounts to 1250 c.c., and to 10 c.c. of it 17 c.c. of the silver solution were added. 50 c.c. of the filtrate required 1.5 c.c. of the sulphocyanate solution; hence the entire filtrate would require $2 \times 1.5=3$ c.c. Therefore, that much silver nitrate solution has been added in excess above that necessary to unite with the $\mathrm{NaCl}$ present in 10 c.c. of the urine.

$17-3=14$ c.c., the amount of silver nitrate solution actually used up by the $\mathrm{NaCl}$ present. Since each c.c. of silver solution equals $10 \mathrm{mg}$. of $\mathrm{NaCl}, 14$ c.c. equals $14 \times 10=140 \mathrm{mg}$. $=0.140 \mathrm{~g}$. NaCl.

$10: 0.140:: 1250: \mathrm{x} . \quad \mathrm{x}=17.50 \mathrm{~g}$. of $\mathrm{NaCl}$ in the 24 hours' urine.

\section{(b). MOHR'S METHOD.}

Rerugents:-

1.-Silver nitrate solution of the same strength as that used in Volhard's method.

2.-A saturated solution of yellow potassium chromate $\left(\mathrm{K}_{2} \mathrm{CrO}_{4}\right)$. This must be free from chlorine and serves as an indicator.

Execution.-Place 10 c.c. of the urine in an evaporating dish, add 2 or 3 drops of nitric acid, about 100 c.c. of water, and $2-3$ drops of the potassium chromate solution. Now add from a burette the silver nitrate solution until a faint red tinge appears. This is due to the formation of silver chromate and shows that all the chlorine has been precipitated and an excess of silver added. Note the amount of silver solution used, deduct 1 c.c. and multiply the difference by 10 ; this gives the number of $\mathrm{mg}$. of sodium chloride in $10 \mathrm{c}$.c. of urine. Calculate the amount present in the 24 hours' urine.

This method is more convenient than the preceding but gives higher results. For that reason $0.5-1$ c.c. is deducted from the observed reading in case the urine is strongly colored. If it is an aqueous solution that is titrated, as in the case of an unknown, the correction should be disregarded. Albumin if present should be removed according to the method given under sugar.

(c). Gravimetric Method.-Place a definite amount of the solution ( 10 c.c. of urine, or 10 c.c. of the unknown) in a small beaker, dilute to about 150 c.c. and acidify with $2-3$ c.c. of $\mathrm{HNO}_{3}$. Then add silver nitrate solution in slight excess and heat to boiling for a few minutes. Filter, and wash the precipitate first with hot water 
slightly acidified with $\mathrm{HNO}_{3}$, then with cold water till free from silver salts. This point is ascertained by collecting a little of the filtrate, as it comes from the funnel, in a test-tube and adding to this a little $\mathrm{HCl}$. Not the slightest cloudiness should result. When this point is reached by repeated washing, place the funnel with its contents in an air-bath at about $100^{\circ}$ for about one hour. While the precipitate is drying, take a clean porcelain crucible, ignite it, set aside in a desiccator to cool, and when cold, weigh.

The precipitate when dry is transferred as carefully as possible to the weighed porcelain crucible. The filter is then rolled up into a small cylinder, a platinum wire is wrapped around it and it is then ignited by means of a small flame. The burning filter should be held over the crucible which should be placed on a black glazed paper so that if any material should drop down it would not be lost sight of. The flame of the burner should be directed against the charred filter paper till it becomes white or grayish. This ash is added to the contents of the crucible. A drop of $\mathrm{HNO}_{3}$ is added to the residue in the crucible and gently warmed. Then a drop of $\mathrm{HCl}$ is added and the whole is heated on a water-bath to dryness. The crucible is now placed on a clay triangle and ignited gently till the residue just begins to fuse at the edges. It is then placed in a desiccator and weighed when cold.

Example. -Total urine $=1450$ c.c. 10 c.c. are taken for analysis:

$$
\begin{aligned}
\text { Crucible }+\mathrm{AgCl}+\text { filter ash } & =12.4554 \\
\text { crucible } & =12.210 \\
\mathrm{AgCl}+\text { filter ash } & =\frac{.2454}{.0001} \\
\text { filter ash } & =\frac{.2453}{\mathrm{AgCl}}
\end{aligned}
$$

$\mathrm{AgCl}: \mathrm{NaCl}::$ weight of $\mathrm{AgCl}$ : weight of $\mathrm{NaCl}$.

$143.5: 58.5:: 0.2453 \quad: \mathrm{x}$

$\mathrm{x}=0.1 \mathrm{~g}$. of $\mathrm{NaCl}$ in $10 \mathrm{c.c}$. of urine.

$10: 0.1:: 1450: y . \quad y=14.5 \mathrm{~g}$. of $\mathrm{NaCl}$ in total urine.

For gravimetric work, if possible, filter paper should be used that has been washed in $\mathrm{HCl}$ and in $\mathrm{HF}$. Such paper is practically pure cellulose and has so little ash that this can be disregarded. If such papers cannot be obtained, then five or ten ordinary filter papers should be ignited as just given and the ash received in a weighed crucible and weighed. The weight of ash from a single filter paper can then be ascertained. 


\section{Determination of Total Sulphuric Acid.}

\section{(a). Volumetrically.}

Reagent.-Dissolve $30.5 \mathrm{~g}$. of barium chloride $\left(\mathrm{BaCl}_{2}+2 \mathrm{H}_{2} \mathrm{O}\right)$ in water and dilute to one liter. Each c.c. $=10 \mathrm{mg}$. of $\mathrm{SO}_{3}$.

Execution.-To 50 or 100 c.c. of the urine add 5-10 c.c. of hydrochloric acid and boil for $1 / 4$ hour in order to break up conjugate sulphates. Then from a burette run in the barium chloride solution, 1 c.c. at a time, as long as a distinct precipitate continues to form; mix and allow the precipitate to subside after each addition; when the formation of a precipitate becomes indistinct filter off after each addition a few c.c. of the liquid and test for sulphuric acid by adding a few drops of barium chloride solution from the burette. If a precipitate forms pour the solution back into the beaker, add more barium chloride, mix and again filter off a small portion. Repeat this addition of reagent and testing until a filtered portion ceases to give a precipitate with barium chloride. Note the amount employed.

The contents of the beaker should be raised to boiling after each addition of barium chloride.

Suppose 17 c.c. of barium chloride does not precipitate all the sulphuric acid while 18 c.c: does. Take the same amount of urine, treat as before, then run in the full amount of barium chloride solution up to the next to the last addition - that is 17 c.c. Mix and test a little of the filtered solution; now add the reagent in smaller amounts than before-about 0.2 c.c. at a time, testing after each addition, till the point of complete precipitation is reached.

Note the amount of barium chloride used. Each c.c. $=10 \mathrm{mg}$. of $\mathrm{SO}_{3}$. Calculate the amount of acid present in the 24 hours' urine.

(b). Gravimetrically.-Place a definite amount of the solution ( 50 or 100 c.c. of urine, or 10 c.c. of the unknown) in a beaker, dilute to about $150 \mathrm{c} . \mathrm{c}$. and acidify with a few drops of $\mathrm{HCl}$. Heat the solution to boiling in order to break up conjugate sulphates. Then add ordinary $\mathrm{BaCl}_{2}$ solution very slowly and with constant stirring till a slight excess has been added. Boil again for some minutes to granulate the precipitate and then set aside for some hours, or better till next day. Filter through paper, the ash of which is known, and wash the precipitate with hot water till the wash-water ceases to give a test for chlorides. 
Dry, transfer the precipitate to a weighed crucible, observing the same care and precaution as given under chlorides (p. 243), and ignite. After ignition moisten the residue in the crucible with a drop of very dilute $\mathrm{H}_{2} \mathrm{SO}_{4}$ : place the crucible on a clay triangle and heat with a very low flame till the fumes of $\mathrm{SO}_{3}$ cease to be given off. Then ignite the crucible gently and place in a desiccator to cool. Weigh the crucible and from the weight obtained, deduct that of the crucible and of the filter ash: the difference is the weight of the $\mathrm{BaSO}_{4}$. Now calculate the amount of $\mathrm{SO}_{3}$ present in the 24 hours' urine.

(c). * Separate determination of simple and conjugate sulphates. - The methods given under $~ \iota$ and $b$ yield the total amount of $\mathrm{SO}_{3}$ present in the urine. To ascertain the amount of $\mathrm{SO}_{3}$ present as simple sulphate and as conjugate sulphate proceed as follows:

Acidulate 50 or 100 c.c. of urine with acetic acid, add $\mathrm{BaCl}_{2}$ in excess and warm on the water-bath for $1 / 2-1$ hour. Then filter through a filter of known ash, wash, dry and ignite as above. This gives the amount of $\mathrm{SO}_{3}$ present as simple sulphate.

The filtrate from the $\mathrm{BaSO}_{4}$ precipitate contains the conjugate sulphates. To break these up, acidulate strongly with $\mathrm{HCl}$ and boil for some time; add more $\mathrm{BaCl}_{2}$ if necessary, allow the precipitate of $\mathrm{BaSO}_{4}$ to settle, then transfer to a filter, wash, dry, and ignite as above. Calculate the amount of $\mathrm{SO}_{3}$ present as conjugate sulphates. Also determine the ratio existing between this and the amount of $\mathrm{SO}_{3}$ in the simple sulphates.

\section{Determination of Phosphoric Acid.}

\section{(1). Iolumetrically.}

Lerengents.-1.-Lrunium arefute solution.-Dissolve $35 \mathrm{~g}$. of crystallized uranium acetate in a liter of water. This solution must be standardized against a standard solution of sodium phosphate so that each c.c. $=5 \mathrm{mg}$. of $\mathrm{P}_{2} \mathrm{O}_{5}$.

For this purpose dissolve $10.0845 \mathrm{~g}$. of crystallized $\mathrm{Na}_{2} \mathrm{HPO}_{4}+$ $12 \mathrm{H}_{2} \mathrm{O}$ in water and make up to 1 liter; each c.c. $=2 \mathrm{mg}$. of $\mathrm{P}_{2} \mathrm{O}_{5}$; 50 c.c. $=0.1 \mathrm{~g}$. of $\mathrm{P}_{2} \mathrm{O}_{5} .20$ c.c. of the uranium acetate solution should equal 50 c.c. of this phosphate solution. If, on titration this is not found to be the case, calculate and make the necessary dilution. The titration is done according to the directions given below.

Sodium phosphate eflloresces very rapidly. The pure crystals should be selected and weighed as rapidly as possible, best in a weighing-bottle. 
2. -3 per cent. acetic acid solution.-If uranium nitrate is used this solution must contain in addition 10 per cent. of sodium acetate in order to combine with the nitric acid which would be set free in the reaction.

3. - Tincture of cochineal, or a solution of potassium ferrocyanide to serve as indicator.

Execution.-Place 50 c.c. of urine, or 10 c.c. of the unknown diluted to $50-100$ c.c., in a beaker or, better still, in a small Erlenmeyer flask; add 5 c.c. of the acetic acid solution, a few drops of the cochineal tincture, and heat to boiling. Now run in from a burette the uranium acetate solution, stirring well, till the liquid becomes faintly but distinctly green and remains so when heated again to the boiling point.

Note the number of c.c. employed; each c.c. $=5 \mathrm{mg}$. of $\mathrm{P}_{2} \mathrm{O}_{5}$. Calculate the amount present in the 24 hours' urine.

Potassium ferrocyanide solution can be used as an indicator instead of cochineal. For this purpose place a series of drops of the solution on a porcelain surface, or on a filter paper, and after each addition of uranium acetate, remove a drop from the beaker, by means a glass rod, and add it to the ferrocyanide; a reddish-brown color indicates the end-reaction,-that uranium acetate has been added in excess.

(b). Gravimetrically.-Phosphoric acid cannot be estimated gravimetrically in solutions containing calcium, iron or other metals precipitable in alkaline solution by this acid, unless it is first of all separated from these metals by means of ammonium molybdate. This step would be necessary, therefore, in order to determine the phosphoric acid in urine gravimetrically. In the absence of such interfering metals, as in the case of the unknown given, the phosphoric acid can be determined directly according to the following method:

Place 10 c.c. of the solution in a, small beaker, dilute to about 50 c.c. and render slightly alkaline with ammonium hydrate. Then add magnesia mixture, drop by drop, till no further precipitation results. While adding this reagent the liquid should be stirred constantly and vigorously, taking care, however, that the rod does not touch the sides of the beaker. Wherever the rod touches the beaker the precipitate adheres tenaciously. Allow the liquid to stand about a quarter of an hour, then add about one-third volume of $\mathrm{NH}_{4} \mathrm{OH}$, mix and set aside for a couple of hours, or over night. Filter through 
a paper of known ash and wash with dilute ammonia (1:4) till the wash-water is free from chlorides. Before testing the wash-water with silver nitrate render it slightly acid with $\mathrm{HNO}_{3}$. Dry, transfer the precipitate to a weighed crucible and ignite the filter according to the directions giren under chlorides (p. 243). Place the crucible on a triangle, apply a small flame and gradually increase: finally ignite for about ten minutes over a Detroit burner or blast-lamp. The residue should be white or nearly so. If it is dark, cool the crucible, add one drop of conc. $\mathrm{H}_{\mathrm{NO}}$ and then place on a water-bath to drive off the acid: finally ignite again for a few minutes. Cool in the desiccator and weigh. Deduct the weight of the crucible and of the filter-ash from the weight obtained. This gives the weight of $\mathrm{Mg}_{2} \mathrm{P}_{2} \mathrm{O}_{7}$. Calculate the amount of $\mathrm{P}_{2} \mathrm{O}_{5}$ present.

In this method the phosphoric acid is precipitated as magnesium ammonium phosphate (triple phosphate). On ignition this is conrerted into magnesium pyrophosphate $\left(\mathrm{Mg}_{2} \mathrm{P}_{2} \mathrm{O}_{7}\right)$ :

$$
\begin{aligned}
& \mathrm{Na}_{2} \mathrm{HPO}_{4}+\mathrm{MgCl}_{2}+\mathrm{NH}_{4} \mathrm{OH}=\mathrm{NH}_{4} \mathrm{NgPO}_{4}+2 \mathrm{NaCl}+\mathrm{H}_{2} \mathrm{O} \text {. } \\
& 2 \mathrm{NH}_{4} \mathrm{MgPO}+\text { ignition }=\mathrm{Ng}_{2} \mathrm{P}_{2} \mathrm{O}_{7}+2 \mathrm{NH}_{3}+\mathrm{H}_{2} \mathrm{O} \text {. }
\end{aligned}
$$

\section{Determination of Glucose.}

(a). Tolumetrically.-By titration with Fehling's solution.

Reagent.-Dissolre 34.64 g. of pure, crystallized copper sulphate in water and make up to $1 / 2$ liter. Likewise dissolve $173 \mathrm{~g}$. of Rochelle salts (potassium sodium tartrate) and $60 \mathrm{~g}$. of sodium hydrate each in 200 c.c. of water. Combine the two solutions and make up to $1 / 2$ liter.

The two solutions thus prepared are usually united and constitute then the so-called Fehling's solution. Inasmuch as Fehling's solution deteriorates on keeping, it is preferable to keep the two constituents separate, mixing them in equal portions just before use.

$$
10 \text { c.c. of Fehling's solution }=50 \mathrm{mg} \text {. of glucose. }
$$

Esccution.-Preliminary trial.-Run 10 c.c. of Fehling's solution from a pipette into a 200 c.c. Erlenmeyer flask (or porcelain evaporating dish), add about 40 c.c. of water and heat to boiling; now run in from a burette the urine or sugar solution 1 or 2 c.c. at a time till the blue color has disappeared and a faint yellow color remains. This can best be seen by holding the flask in an inclined position before a window. Allow the precipitate to partly subside after each addi- 
tion, the better to see the color. When decoloration takes place note the amount of urine or solution used and calculate the amount of sugar present in 100 c.c.

This preliminary trial will give approximately the strength of the sugar solution used. The above factor for Fehling's solution (10 c.c. $=50 \mathrm{mg}$. of glucose) holds true only for-solutions of a certain strength, namely about 0.5 per cent. glucose. Having ascertained the approximate strength of the sugar solution dilute this so that it will contain about 0.5 per cent. of sugar. Note carefully the quantity of the sugar solution taken and the volume to which it is diluted.

Final determination.-Calculate approximately the number of c.c. of the diluted sugar solution necessary to reduce 10 c.c. of Fehling's solution. Now measure out 10 c.c. of Fehling's solution, dilute and heat as above. Then run in nearly the calculated amount of diluted sugar solution, heat to boiling and examine for the end reaction according to the directions given above. If the solution contains unreduced copper, that is, is colored blue, add $1 / 2$ c.c. of the sugar solution, raise to boiling and examine as before. Continue the addition of the sugar solution, in portions of $1 / 2$ c.c. till the blue color just disappears. In case of doubt filter some of the liquid into a test-tube. If this is nearly full and held against a white surface a trace of blue color can be readily detected. Another procedure is to acidulate a few c.c. of the filtrate with acetic acid and then add a drop or two of potassium ferrocyanide. If copper is present a brown precipitate or coloration will result.

Example.-On preliminary trial 10 c.c. of Fehling's solution required between 5 and 6 c.c. of the urine. This represents 0.8 to 1.0 g. of sugar in 100 c.c. The original solution is therefore about twice as strong as it should be. Consequently 50 c.c. of this solution were diluted to 100 c.c.

On repeating the titration 10 c.c. of Fehling's solution required 10.3 c.c. of the diluted urine. This amount, therefore, contains 50 $\mathrm{mg}$. of glucose. The amount of sugar present in the entire solution is then ascertained by the proportion: $10.3: 50:: 100: x . \quad x=485 \mathrm{mg}$. $=0.485 \mathrm{~g}$. of glucose in $100 \mathrm{c} . \mathrm{c}$. of the diluted or in $50 \mathrm{c.c}$. of the undiluted urine. If now the total 24 hours' urine is 4500 c.c. the amount of sugar present will be given by the proportion:

$$
50: 0.485:: 4500: x . \quad x=43.65 \mathrm{~g} \text {. of glucose. }
$$


If albumin is present this should be removed, since it combines with copper and also interferes with the settling of the precipitate. This can best be done as follows: To 100 c.c. of the urine add $10-15$ c.c. of saturated salt solution, acidulate distinctly with a few drops of acetic acid and boil several minutes, then cool, make up to 100 c.c. and filter through a dry filter.

Inasmuch as sugar solutions are prone to fermentation the quantitatice examination should be made as early as possible.

\section{(b)*. By fermentation.}

1.-From the difference in specific gravity, before and after fermentation. Determine the specific gravity of the fresh urine and reduce this to $15^{\circ}$. Take 200 c.c., add about 1 gram of yeast and set aside in a bottle closed with a perforated stopper for $24-48$ hours at a temperature of $25^{\circ}-30^{\circ} \mathrm{C}$. Then filter off a small portion and test with Fehling's solution. If reduction takes place continue the fermentation till all the sugar has disappeared. J.Then filter the urine, determine the specific gravity and reduce to $15^{\circ}$. The difference between the two, multiplied by 230 (Roberts' factor), gives the amount of sugar in grams in 100 c.c. Calculate the amount in the 24 hours' urine.

The results obtained by this method are quite satisfactory, especially if the urinometer reads to four decimal places, or the specific gravity is determined by the picnometer.

The above factor holds true in the case of urines containing a small amount of sugar. If the specific gravity is high it is advisable to dilute the urine as given on p. 2 ว.

2. - From the amount of carbonic acid formed.

Einhorn's saccharimeter and similarly constructed in. struments can be used.

Sugar on fermentation with yeast breaks up into alcohol and carbonic acid:

$$
\mathrm{C}_{6} \mathrm{H}_{12} \mathrm{O}_{6}=2 \mathrm{C}_{2} \mathrm{H}_{6} \mathrm{O}+2 \mathrm{CO}_{2} \text {. }
$$


If the fermentation takes place in a U-shaped tube, one arm of which is closed and graduated the carbonic acid given off will accumulate in the closed arm and the volume of gas, or the percentage of sugar that this corresponds to, can be read off directly. For Einhorn's instrument 10 c.c. of urine are taken, $1 \mathrm{~g}$. of yeast added, mixed thoroughly and the mixture is filled into the fermenting tube. This is set aside for 15-20 hours. The volume of gas gives directly the percentage of sugar present. If the urine is rich in sugar, that is, possesses a high specific gravity, it should be diluted, as follows:

Specific gravity 1.018-1.024, with 2 volumes of water.

$$
\begin{array}{rrrrrr}
1.024-1.028, & 6 & 5 & 6 & 6 & 6 \\
1.028-1.038, & 6 & 10 & 6 & 6 & \text { "6 }
\end{array}
$$

Even with this precaution the results are far from satisfactory. The rate of fermentation is influenced by the temperature. The volume of the gas is affected by temperature and barometric pressure. The method, therefore, cannot be recommended.

3.-From the amount of alcohol formed.

(c)*. Polarization method.-This gives reliable results, though a trifle low. The expense of the instrument is such as to preclude its general use (see p. 35).

$(d)^{*}$. Gravimetrically. - The amount of sugar present in a solution may be ascertained by collecting the $\mathrm{Cu}_{2} \mathrm{O}$ formed on heating with Fehling's solution and converting this into metallic copper by heating it in a current of hydrogen. Allihn's tables give the amount of glucose corresponding to the weight of copper found. 


\section{Determination of Urea.}

1.-Liebig's Method. - This is more nearly an estimate of the total nitrogen present in the urine.

Reagents.-1.-Hercuric nitrate solution.-Dissolve $77.2 \mathrm{~g}$. of mercuric oxide (or $71.48 \mathrm{~g}$. of mercury) in nitric acid and evaporate to drive off the excess of acid, then add water gradually, stirring well, and dilute to one liter. Each c.c. should equal $10 \mathrm{mg}$. of urea. The solution should be standardized against a 2 per cent. urea solution.

2.-Baryta mixture.-Combine one rolume of cold saturated barium nitrate with two rolumes of cold saturated barium hydrate. This is used to remore phosphoric acid.

3.--Solution of sodium carbonate containing $53 \mathrm{~g}$. of the salt in one liter of water. This serves to indicate the end reaction.

Execution.-To 40 c.c. of the urine add 20 c.c. of baryta mixture, mix well and filter through a dry filter. Place 15 c.c. of the clear filtrate, which corresponds to 10 c.c. of the original urine, in a small beaker and run in the mercuric nitrate solution from a burette. 1 c.c. at a time, stirring well and testing with sodium carbonate after each addition. This is best done by placing a series of drops of sodium carbonate by means of a glass rod on a flat porcelain surface, and after each addition of reagent adding a drop of the mixture from the beaker to the drop of sodium carbonate. Continue the addition of the mercury solution till a distinct yellow coloration is produced by the sodium carbonate. It is well to wait a minute for the development of the color before adding more reagent.

Now repeat the titration with a new portion, running in the full amount up to the point short of the yellow color and from this on add 0.2 c.c. at a time till the end reaction appears. Note the number of c.c. required: each c.c. $=10 \mathrm{mg}$. of urea. Calculate the amount in the 24 hours" urine.

If an aqueous "unknown" of urea is to be titrated, take 10 c.c. and add the mercuric solution to this. The baryta mixture should not be added, when no phosphates or sulphates are present.

If much albumin is present it should be removed by the aid of heat and acetic acid. The $\mathrm{NaCl}$ method is not applicable. 
The above is the method as ordinarily and most easily employed. More exact results can be obtained if the chlorides present are removed by precipitation with just sufficient silver nitrate. Why is this desirable?

Again, in the above method as seen from the following equation, nitric acid is set free.

$$
\begin{aligned}
& 2 \mathrm{CO}\left(\mathrm{NH}_{2}\right)_{2}+4 \mathrm{Hg}\left(\mathrm{NO}_{3}\right)_{2}+3 \mathrm{H}_{2} \mathrm{O}= \\
& \qquad \begin{array}{l}
2 \mathrm{CO}\left(\mathrm{NH}_{2}\right)_{2} \cdot \mathrm{Hg}\left(\mathrm{NO}_{3}\right)_{2} \cdot 3 \mathrm{HgO}+6 \mathrm{HNO}_{3} .
\end{array}
\end{aligned}
$$

This free acid tends to alter the composition of the precipitate and to dissolve it, hence the solution should be carefully neutralized.

2.- *Hüfner's method.-This depends upon the fact that urea is decomposed by an alkaline solution of sodium hypobromite into nitrogen, carbonic acid and water.

Reagent.-Alkaline hypobromite solution. Dissolve 100 g. of sodium hydrate in 250 c.c. of water and when cold add slowly and cautiously 25 c.c. of bromine. Keep in a cool, dark place.

The above quantity of reagent is necessary when using Hüfner's apparatus, but when the small modified form, as that of Doremus, is used it is unnecessarily large. In that case it is better to add 1 c.c. of bromine to 10 c.c. of the $\mathrm{NaOH}$ solution just before use.

Execution.-Place 1 c.c. of the urine in the lower bulb of Hüfner's apparatus, then fill it full of water up to the farther end of the perforation in the stopper. Now close the stopper. Fill the remainder of the apparatus and the measuring tube with the reagent. Close the mouth of the tube with a finger and invert over the apparatus so that no air enters, and place it into position. When this is done open the stop-cock; when the reagent passes into the bulb where the urine is and sets nitrogen free. This collects above in the measuring tube. In about a half hour the operation is completed and the volume of gas can be measured, using the proper precautions. 
From the volume of nitrogen the weight of the urea can be calculated according to the formula:

$$
\mathrm{G}=\frac{\nabla\left(\mathrm{b}-\mathrm{b}^{\prime}\right)}{354.5+760(1+0.003665 \mathrm{t})}
$$

$\mathrm{G}=$ weight of urea; $v=$ volume of gas; $b=$ barometric pressure; $b^{\prime}$ aqueous tension at $t^{\circ} ; t=$ temperature.

The above procedure is too expensive for ordinary clinical work. The principle, however, can be utilized advantageously in the small modified apparatus, or ureometer of Doremus. The results, however, are usually a trifle low. The instrument is filled to the bend with a mixture $\mathrm{cf}$ equal parts of hypobromite and water. 1 c.c. of the urine is then introduced by means of a nipple pipette. The volume of nitrogen given off gives directly the amount of urea in 1 c.c. of urine.

3.- Other methods for determination of urea are based upon its decomposition into ammonia and carbonic acid, but for ordinary purposes they are not applicable.

The same is true of Mörner and Sjöqvist's method, in which all the nitrogen constituents of urine, except urea, are precipitated by addition of $\mathrm{BaCl}_{2}$, baryta mixture, alcohol and ether. The filtrate is concentrated and in it the nitrogen is determined by the Kjeldahl process.

4.-The specific gravity affords an approximate idea of the amount of urea present, provided sugar and albumin are absent and the volume of urine and the amount of chlorides are about normal.

A specific gravity of 1.010 indicates about 1.0 per cent. of urea; 1.015 indicates about 1.5 per cent.; and 1.020 indicates a little less than 2 per cent. of urea.

Above this point the variation is too great to be of any value. 


\section{Determination of Uric Acid.}

1.-Method of Heintz.-Place 200 c.c. of the filtered urine in a beaker, add 5 c.c. of conc. hydrochloric acid, mix, cover and set aside for 24-48 hours in a cool place. Filter through a previously dried and weighed filter, transfer the crystals to the filter, wash with small portions of cold water till the chlorides are removed, then

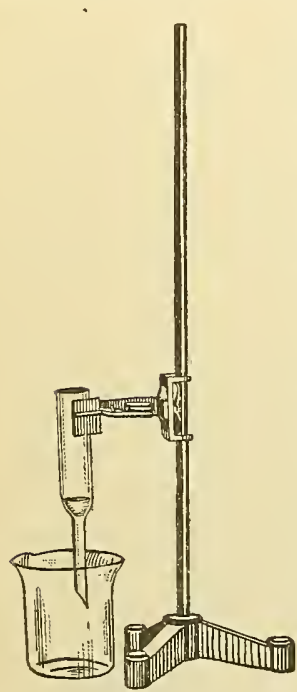

Fig. 5. dry at $100-110^{\circ}$ and weigh. The difference in the two weighings represents the uric acid. But as uric acid is not wholly insoluble in water, some of it is in solution. Therefore, measure the filtrate and wash-water and for every 100 c.c. add 0.0048 g. to the amount of uric acid as found above. Now calculate the total amount in the 24 hours' urine. By making this correction the method is as accurate as the following one. If urates are present they should be brought into solution by the aid of heat or by the addition of sodium hydrate. Albumin must be removed.

Filter paper should not be weighed in the open air but should be placed in a weighing bottle or well corked test-tube.

Instead of using a filter paper for the above or similar purposes it is preferable to use an asbestos filter in connection with a Chapman air-pump. The filter is shown in the accompanying figure (Fig. 5).

\section{2.- Method of Ludwig.}

Reagents.-1.-Ammoniacal silver nitrate solution. Dissolve $26 \mathrm{~g}$. of silver nitrate in water, add ammonium hydrate till the precipitate which first forms redissolves, then dilute to 100 c.c.

2.-Magnesia mixture.-Dissolve $10 \mathrm{~g}$. of magnesium chloride in water, add ammonium hydrate in strong excess and then ammonium chloride till the precipitate is redissolved. Dilute to 100 c.c. The solution should be strongly ammoniacal.

3.-Sodium or potassium sulphide solution.-Dissolve $15 \mathrm{~g}$. of potassium hydrate (or $10 \mathrm{~g}$. of sodium hydrate), free from nitrous and nitric acids, in 100 c.c. of water. Saturate one-half the solution 
with hydrogen sulphide which forms KHS. Then combine the two solutions forming $\mathrm{K}_{2} \mathrm{~S}$.

Execution.-To 100 c.c. of urine add a mixture of 10 c.c. of the silver solution and 10 c.c. of magnesia mixture and mix. If when making this mixture silver chloride is thrown down more ammonia is to be added; if magnesium hydrate precipitates more ammonium chloride is needed. Allow the precipitate to settle, then filter and wash 2-3 times with water to which a little ammonia has been added. When the liquid has drained, transfer by means of a glass rod the moist precipitate to the beaker in which the precipitation was made, place the beaker under the funnel and wash the residue on the filter with a boiling mixture of 10 c.c. of potassium sulphide solution and 10 c.c. of water. Heat on a water-bath for some time and when the entire precipitate is black, filter through the filter previously used. Collect the filtrate in a small dish and wash the filter with hot water. To the combined filtrate and wash-water add 5 c.c. of dilute hydrochloric acid and concentrate to $10-15$ c.c. Set aside to cool: in about half an hour all the uric acid will have crystallized out. Filter through a glass-wool or asbestos filter, or through paper previously dried at 110 : and weighed. Wash with a little water, then dry and wash three times with carbon bisulphide to remore sulphur, then with ether. [Finally dry at $110^{\circ}$ and weigh. The difference between the two weighings gives the amount of uric acid. Calculate the amount present in the 24 hours urine.

If albumin is present it should be remored according to the method on p. 249.

3. - Haycraft's method.-This is a volumetric method based upon the same principle as the preceding and gives somewhat higher results. The uric acid is thrown out of solution and the precipitate is washed as above. Then it is dissolved in nitric acid and the dissolved silver titrated with a so potassium sulphocyanate solution (see p. 241). Each c.c. used $=3.36 \mathrm{mg}$. of uric acid.

4. - Crapel's method. - This method is the complement of Haycraft's. That is, instead of estimating the silver in the precipitate, the silver in the filtrate is determined and the difference between this amount and the total amount added 
gives the amount of silver that combined with uric acid. The results are likewise higher than by Ludwig's method.

5.-Hopkins' method.-This depends upon the fact that uric acid is completely precipitated from urine on saturation with $\mathrm{NH}_{4} \mathrm{Cl}$ as ammonium urate. Furthermore, uric acid can be titrated in warm, $\mathrm{H}_{2} \mathrm{SO}_{4}$ solution with $\mathrm{KMnO}_{4}$. Instead of $\mathrm{NH}_{4} \mathrm{Cl}$ other ammonium salts can be employed and the addition of 10 per cent. of these salts is sufficient. The method as modified by Folin is as follows:

To 100 c.c. of the urine add $10 \mathrm{~g}$. of $\left(\mathrm{NH}_{4}\right)_{2} \mathrm{SO}_{4}$, stir till dissolved, then add $\mathrm{NH}_{4} \mathrm{OH}$ to slight but distinct alkaline reaction. The ammonium urate is allowed to settle for two hours. The solution is filtered and the precipitate washed with a 10 per cent. $\left(\mathrm{NH}_{4}\right)_{2} \mathrm{SO}_{4}$ solution, till free from chlorides. It is then rinsed into a small beaker, diluted to 100 c.c. and 15 c.c. of concentrated $\mathrm{H}_{2} \mathrm{SO}_{4}$ added. Heat to $60^{\circ}$, then titrate immediately with ${ }_{20} \mathrm{KMnO}_{4}$, Each c.c. of the $\mathrm{KMnO}_{4}$ solution corresponds to $3.75 \mathrm{mg}$. of uric acid. A correction of $1 \mathrm{mg}$. is to be added to the end result.

The method is rapid, easy of execution and gives almost as good results as that of Ludwig. The $\mathrm{KMnO}_{4}$ solution can be prepared by dissolving $1.578 \mathrm{~g}$. of the pure crystals in water and diluting to one liter. It should then be tested against $\frac{N}{10}$ oxalic acid. Ten c.c. of the latter are placed in a beaker, diluted with a little water, $\mathrm{H}_{2} \mathrm{SO}_{4}$ added and the mixture warmed to $60^{\circ}$. The $\mathrm{KMnO}_{4}$ solution is then run in to a permanent pink color. Twenty c.c. of the latter should be consumed; if not, calculate the factor. Thus, 10 c.c. of $\frac{N}{10}\left(=20\right.$ c.c. $\left.\frac{N}{20}\right)$ oxalic acid required 19.6 c.c. of the $\mathrm{KMnO}_{4} ; \frac{20}{19.6}=1.0204$, the factor of the $\mathrm{KMnO}_{4}$ solution. That is to say, the number of c.c. of this $\mathrm{KMnO}_{4}$ used multiplied by this factor gives the number of c.c. of $\frac{\mathrm{N}}{20}$ $\mathrm{KMnO}_{4}$ that this corresponds to.

\section{Alloxuric Bodies and Bases.}

Uric acid and the nuclein bases are known to possess an alloxan and an urea group in their molecule. Moreover they are all precipitated on boiling with a solution of copper sulphate and a reducing agent (see p. 144, Exp. 4). The term alloxuric bodies has been given to all those consti- 
tuents of urine which contain the two groups mentioned. Deducting from the alloxuric bodies the uric acid present in the urine leaves the alloxuric bases. The latter, therefore, include the nuclein bases, as well as other related compounds which bave not as yet been isolated from the urine.

The following members of the xanthin, or nuclein group may be obtained from urine (see p. 153): Xanthin, hypoxanthin, guanin, paraxanthin, heteroxanthin, methyl xanthin, carnin, episarkin and adenin. They are ordinarily present in very small amount but may be appreciably increased in disease, as in leukæmia. The separation of these bases is a long and tedious process. For the details of isolation see Vaughan and Nory-Ptomaïns and Leucomaïns.

\section{ESTIMATION OF ALLOXURIC BODIES AND BASES.}

The reagents employed are a 13 per cent. solution of copper sulphate: a solution of sodium acid sulphite $(1: 2)$ : and a 10 per cent. solution of barium chloride. The method is as follows:

Place 100 c.c. of the albumin free urine in a beaker and boil, then add 10 c.c. of the copper sulphate solution and 10 c.c. of the sodium acid sulphite solution and boil a few minutes. Then add 5 c.c. of the barium chloride solution in order to cause the precipitate to settle more readily. Let stand two hours. Then transfer to a small plaited filter and wash five times with water heated to $60^{\circ}$. Now place the filter and contents in a Kjeldahl flask, and determine the nitrogen present according to the Kjeldahl method (p. 258).

A blank experiment must be made, using a clean filter paper, instead of the one with the precipitate. The number of c.c. of decinormal ammonia which is found in this blank experiment must be deducted from the number of c.c. found above. The difference represents the number of c.c. of deci-normal ammonia formed from the alloxuric bodies in 100 c.c. of the urine. Therefore, this number multiplied by the deci-normal factor of nitrogen gives the nitrogen in the alloxuric bodies.

In another portion of the urine determine the amount of the uric acid according to the Salkowski-Ludwig method as given on p. 255 . Calculate the amount of uric acid contained in 100 c.c. of the urine, and then the nitrogen contained in this amount of uric acid. 
Subtracting the nitrogen of uric acid from the nitrogen of alloxuric bodies gives the nitrogen of alloxuric bases in 100 c.c.

The ratio of the nitrogen of alloxuric bases to the nitrogen of uric acid is about 1 to 4 in normal urine. In leukæmia it may be 1 to 1 .

The class is divided into sets of three. One student determines the alloxuric bodies: another the uric acid according to Ludwig, and the third determines total nitrogen. These three determinations are made with the same urine.

\section{Determination of Total Nitrogen.}

1.- Kjeldahl's method.-This method is based upon the fact that nitrogenous substances on prolonged heating with $\mathrm{H}_{2} \mathrm{SO}_{4}$ are completely oxidized and the nitrogen present is converted into ammonia.

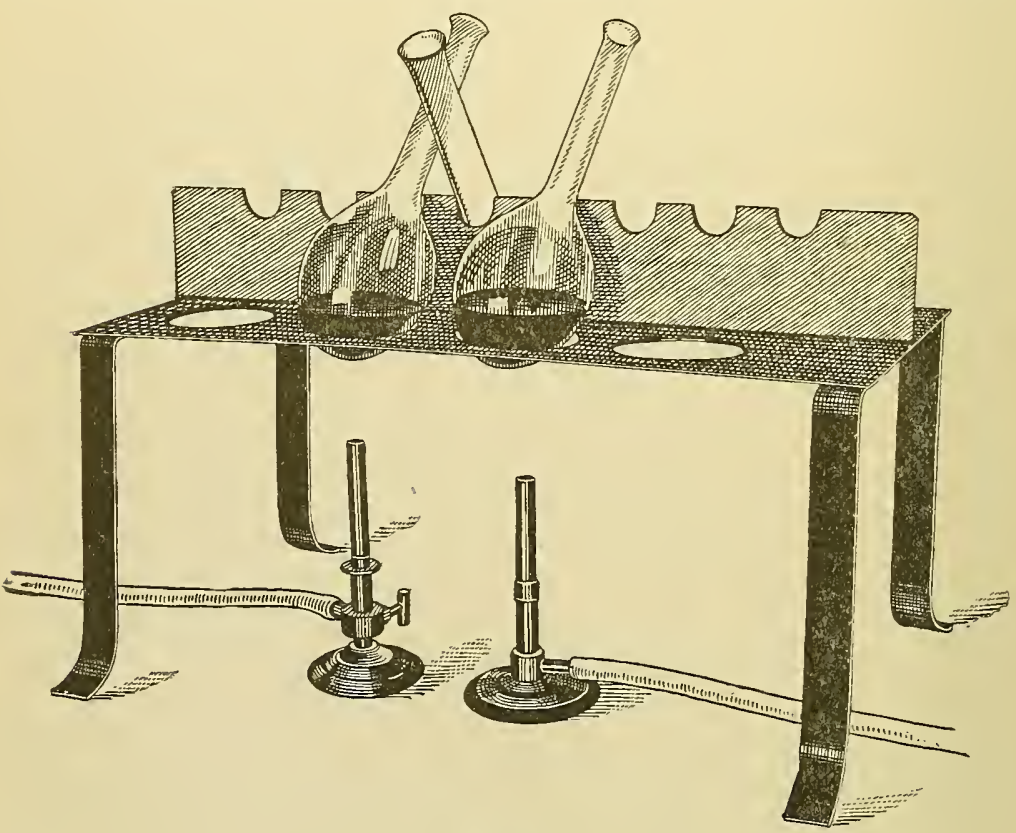

Fig. 6.

The ammonium sulphate is then decomposed by distillation with an alkali and the ammonia that distils over is collected in a known quantity of an $\frac{N}{10}$ acid. On determining by titration with $\frac{N}{10}$ alkali 
the amount of acid left unneutralized, the difference between this and the amount taken represents the number of c.c. of $\frac{\mathrm{N}}{\mathrm{N}} \mathrm{NH}_{3}$ distilled: multiplying this by the $\frac{\mathrm{N}}{10}$ factor of $\mathrm{N}$, the amount of $\mathrm{N}$ present is at once obtained.

The method is carried out as follows: Place 5 c.c. of urine in a 250 c.c. Kjeldahl flask. (Fig. 6). Add 15 c.c. of $\mathrm{H}_{2} \mathrm{SO}_{4}$. and $1 / 7 \mathrm{~g}$. of powdered $\mathrm{CuSO}_{4}$ : heat on a wire gauze under the hood till foaming ceases, then add $10 \mathrm{~g}$. of powdered $\mathrm{K}_{2} \mathrm{SO}_{4}$ and continue gently boiling till the

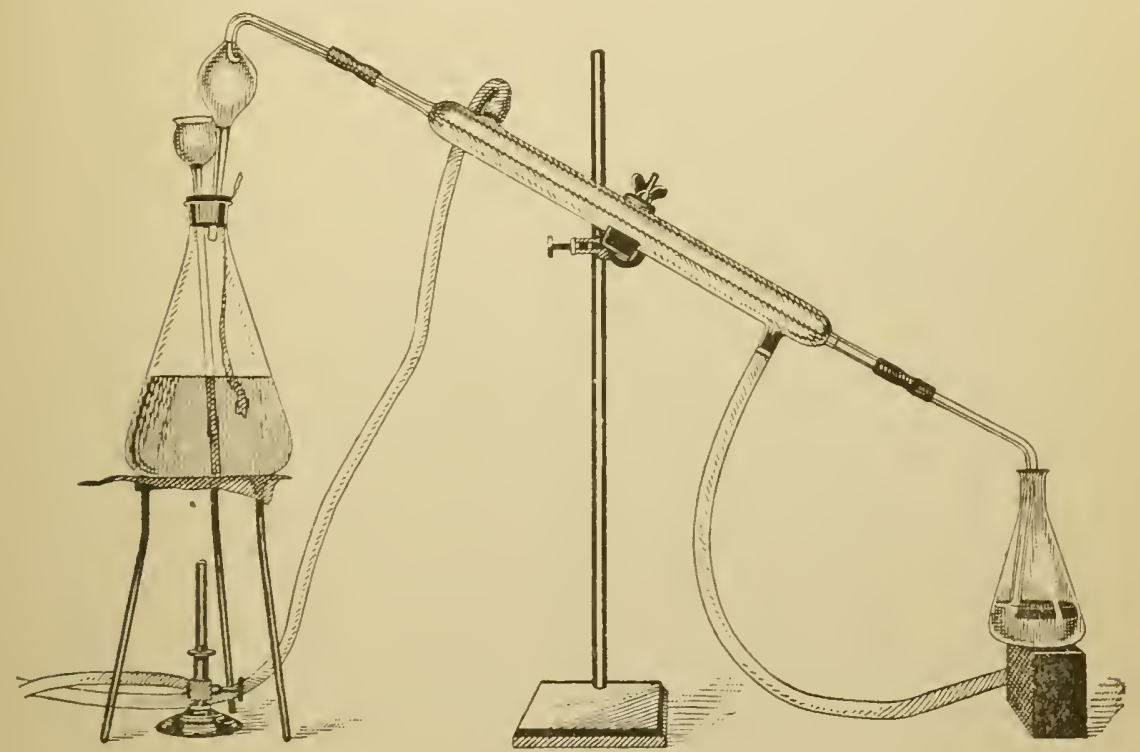

Fig. 7 .

liquid is light green. Finally add a little powdered $\mathrm{KMnO}_{4}$, on the point of a knife. in order to complete the oxidation, and heat till the liquid is light green in color. Allow to cool, then transfer the contents to a liter Erlenmeyer flask. Rinse out the digestion flask several times with water and add this to the acid solution. Dilute the contents of the flask to about 500 c.c. and cool. Add a little powdered talc on the end of a knife. Insert in the neck of the flask a double perforated rubber stopper, provided with a Reitmaier bulb and a thistle tube. The end of the latter should reach nearly to the bottom of the flask. A long strip of red litmus paper should be suspended from the neck of the flask and should extend down into the liquid. Connect the free end of the Reitmaier bulb with a condenser. 
The lower end of the condenser is connected with a bent tube which extends down into the liquid of the receiving flask. (Fig. 7). This should have about 500 c.c. capacity and contains 50 c.c. of $\frac{N}{10}$ oxalic acid, which will unite with the $\mathrm{NH}_{3}$ that will be distilled off. When all is in readiness pour into the flask, through the tube, strong $\mathrm{NaOH}$ solution (1:2) until the liquid is decidedly alkaline. About 50-60 c.c. will be required. Heat the large flask and distil over about 200 c.c. Then replace the receiver by a flask containing 10 c.c. of ${ }_{1}^{\mathrm{N}}$ oxalic acid and some water and continue the distillation till about 100 c.c. of distillate passes over.

To each of the receivers now add a few drops of alcoholic rosolic acid and titrate with $\frac{\mathbb{N}}{10} \mathrm{NaOH}$ to a deep pink color. The second flask serves as a check and should be free, or nearly free, from ammonia. The difference between the number of c.c. of oxalic acid employed and the number of c.c. of $\frac{\mathrm{N}}{10} \mathrm{NaOH}$ necessary to neutralize the distillate gives the number of c.c. of $\frac{\mathrm{N}}{10} \mathrm{NH}_{3}$ given off in the distillation.

A blank experiment with 15 c.c. of $\mathrm{H}_{2} \mathrm{SO}_{4}$ and the other reagents, but with no urine added, should be carried out exactly in the same manner as described above with urine, in order to ascertain the amount of ammonia formed from the nitrogen that may possibly be present in the reagents. The number of c.c. of $\frac{\mathrm{N}}{10} \mathrm{NH}_{3}$ thus found should be subtracted, as a correction, from the total number of c.c. of ${ }_{10}^{\mathrm{N}} \mathrm{NH}_{3}$ given off in the experiment with urine. The difference represents the number of c.c. of $\frac{\mathrm{N}}{10} \mathrm{NH}_{3}$ formed from the nitrogen actually present in the urine. This difference multiplied by the $\frac{\mathrm{N}}{10}$ factor of nitrogen $(0.0014)$ gives the amount of nitrogen contained in 5 c.c. of urine. The amount of total nitrogen present in 100 c.c. or in the 24 hours' urine can be readily calculated.

2.- Dumas' method.-Five c.c. or more of the urine are acidulated with sulphuric acid and evaporated to dryness in a copper boat. This is then filled with copper oxide and placed in a combustion tube and heated. The nitrogen present is set free and is collected in a suitable apparatus and measured. From this, the amount of nitrogen in the total urine can be calculated.

3.—* Varrentrapp - Will's method.-This gives good results but it is more expensive and takes more time than the Kjeldahl method and hence has been supplanted by it. The residue from 5 c.c. of urine is mixed with soda-lime 
and ignited. The nitrogen present is converted into ammonia and this is estimated by receiving it into io acid solution.

4.-Liebig's method for titration of urea gives results which correspond very closely to the total nitrogen present, rather than to urea itself. (See p. 251).

\section{Determination of Albumin (and Globulin).}

1.-Scherer's method.-Place 100 c.c. of the clear urine in a small beaker. If much albumin is present, take less and dilute. If the reaction is not distinctly acid, acidulate slightly with acetic acid and heat on the water-bath for about $1 / 2$ hour. The beaker should be covered and so placed that the lower half containing the urine passes through the ring of the water-bath. Heat till a coagulum of coarse floccules forms. The addition of a minute amount of acid aids this, but a larger amount must be avoided. (Compare Exp. 8, p. 41; also Exp. 3, p. 110). Filter through a weighed filter, preriously dried at $120-130^{\circ}$, and wash with hot water till the precipitate ceases to give the chloride reaction. Fill the funnel several times with absolute alcohol, then twice with ether (to remore fats), and dry at $120-130^{\circ}$ till the weight is constant.

It is well to make a few preliminary tests on the completeness of coagulation. For this purpose test the reaction and if acid. place some of the urine in a test-tube and heat in a water-bath till coagulated, then for a few minutes in the flame and filter. If the filtrate is cloudy or on testing with acetic acid and potassium ferrocyanide gives a cloudiness, the urine is not sufficiently acid and a drop or so of acetic acid must be added. In this way make one or more tests till complete precipitation is obtained, and then acidulate to the proper degree the amount of urine taken for the estimation.

Bearing in mind the fact that $\mathrm{NaCl}$ favors the coagulation of albumin with acetic acid (Exp. 8, p. 41), it is well to add to the urine which has been measured out $10-15$ per cent. of common salt.

2. - Densimetric met/ood. - In this method the specific gravity of the urine before and after coagulation is determined and the difference between the specific gravities 
multiplied by 400 (Zahor's factor) gives the number of grams of albumin in 100 c.c.

The amount of acetic acid necessary to coagulate is ascertained as given above. This amount is then added to the urine and the specific gravity determined. The coagulation should be carried on in such a manner that no water is lost by evaporation, and for that purpose the urine is placed in a bottle provided with a rubber stopper and clamp (beer bottle). This is now immersed in water and heated to boiling for 10-15 minutes. The flask is then cooled, the contents filtered and the specific gravity of the filtrate determined.

The urinometers employed should read to four decimal places, or better still, the specific gravity should be determined by means of a picnometer. The results are excellent and approach closely the gravimetric results.

3.—*Roberts-Stolnikoff's method.-A solution containing $3 \mathrm{r} / 3 \mathrm{mg}$. of albumin in 100 c.c. gives a faint but distinct reaction with Heller's test in 2-3 minutes. Hence dilute the urine under examination till it gives the test under these conditions. Then calculate the amount of albumin present in the 24 hours' urine. The results are fairly good for clinical purposes.

\section{4.-_Esbach's albuminometer.}

Reagent. $-10 \mathrm{~g}$. of picric acid and $20 \mathrm{~g}$. of citric acid are dissolved in 1 liter of water.

Execution.-The instrument is filled with urine to the $\mathrm{U}$ mark, then with the reagent to the mark $R$. The tube is closed with a stopper, inverted several times and then set aside in an upright position for 24 hours. Read off the height of the precipitate in the tube. The figures indicate grams of albumin in one liter.

If much albumin is present the urine should first be diluted. The specific gravity should be 1.010 or lower. 
The results obtained by this method are only roughly approximate. The method possesses the same value as Einhorn's saccharimeter.

XIV. *Separate Determination of Globulin and Albumin.

1.-Hammarsten's method.-Measure out 25-100 c.c. of the urine, according to the amount of albumin present and place in a beaker. Render slightly alkaline with potassium hydrate, filter to remove phosphates and wash. Add the wash-water to the filtrate. Neutralize with acetic acid. For 100 c.c. of solution add $120 \mathrm{~g}$. of pulverized magnesium sulphate, warm to $30^{\circ}$ and stir well; then set aside for 24 hours in the cold. Filter through a weighed filter, previously dried at $110^{\circ}$; rinse out the beaker with saturated magnesium sulphate solution and wash with the same till the filtrate ceases to give a reaction for albumin on heating with acetic acid. Then dry the precipitate for several hours at $110^{\circ}$ to coagulate the globulin, cool and wash with hot water till all magnesium sulphate is removed. Then wash with alcohol and ether, dry at $110^{\circ}$ till the weight is constant, cool and weigh. Subtract the weight of the filter; the difference represents the weight of the globulin together with some inorganic matter. Hence, ignite the filter and weigh the ash. Subtract the weight of the ash from the weight of the globulin as first found; this gives the weight of the globulin. Calculate the amount in the 24 hours' urine.

If the globulin and albumin are determined by Scherer's method, the difference between the two results gives albumin.

2.-Pohl's method.-In this the globulin is precipitated with an equal volume of a saturated solution of ammonium sulphate and the precipitate is washed with a semi- 
saturated solution of the same. After that the process is the same as that given above.

The results are about the same as those obtained by Hammarsten's method; moreover, the method possesses the advantage that the filter does not clog.

\section{XV. *Determination of Albumose.}

From 50 to 100 c.c. of the urine, according to the amount of albumose present, are taken for this purpose. Albumin and globulin, if present, must first be removed by the aid of acetic acid and heat. To facilitate the precipitation $10 \mathrm{~g}$. of $\mathrm{NaCl}$ can be added; the liquid in that case must be filtered boiling hot, and the precipitate washed with boiling water. The filtrate and wash-water are concentrated to about the original volume, cooled and strongly acidulated with sulphuric acid, then treated with an acid solution of sodium phosphotungstate (1 volume of dilute $\mathrm{H}_{2} \mathrm{SO}_{4}$ and 3 volumes of sodium phosphotungstate solution) as long as a precipitate forms. The precipitate is filtered and washed with dilute sulphuric acid (1:3); the filter with its contents is placed in a flask and the nitrogen determined by the Kjeldahl method. The proper allowance for the blank is made. The weight of nitrogen multiplied by 6.25 gives the weight of the albumose. 
Average Composition of Urine (AFTER SCHOTTEN). 1500 c.c.

Average quantity per 24 hours is placed at about

I. Normal Constituents. $-60 \mathrm{~g}$.

A._Organic, -3ǎ g.

Urea, $30 \mathrm{~g}$.

Uric acid, $0.6 \mathrm{~g}$.

Creatinin, $0.8 \mathrm{~g}$.

Xanthin compounds, oxalic acid, oxaluric acid, volatile fatty acids, lactic acid, glycerinphosphoric acid, sulphocyanic acid.

Hippuric acid; phenol-, cresol-, pyrocatechin-, indoxyl- and skatoxyl-sulphuric acids; oxyphenylacetic acid; hydrocumaric acid.

Urine pigments.

Ferments; sulphur and nitrogen containing substances; substances of unknown composition free from sulphur and nitrogen. Total a few grams.

B-Inorganic, $-25 \mathrm{~g}$.

Sodium chloride, $15 \mathrm{~g}$.

Sulphuric acid, $2.5 \mathrm{~g}$.

Phosphoric anhydride, $2.5 \mathrm{~g}$.

Nitric acid, $0.1 \mathrm{~g}$. (or less).

Potassium oxide, $3.0 \mathrm{~g}$.

Ammonia, $0.7 \mathrm{~g}$.

Magnesium oxide, $0.5 \mathrm{~g}$.

Calcium oxide, $0.3 \mathrm{~g}$.

Iron, $0.01 \mathrm{~g}$. (or less).

II. Abnormal, or Pathological Constituents.

Albumin,

Globulin,

Albumose,

Pepton, ?

Mucin,

Grape sugar,

Milk sugrar,

Laevulose,

Inosite,

Aceton, acetacetic acid,
ß-Oxybutyric acid,

Blood, blood pigments, Melanin and other pigments, Bile, pigments and acids, Fat, cholesterin, lecithin, Leucin, tyrosin,

Alkapton (uroleucinic and homogentisinic acids).

Cystin, putrescin, cadaverin, Hydrogen sulphide. 
Analysis of 24 Hours' Urine.

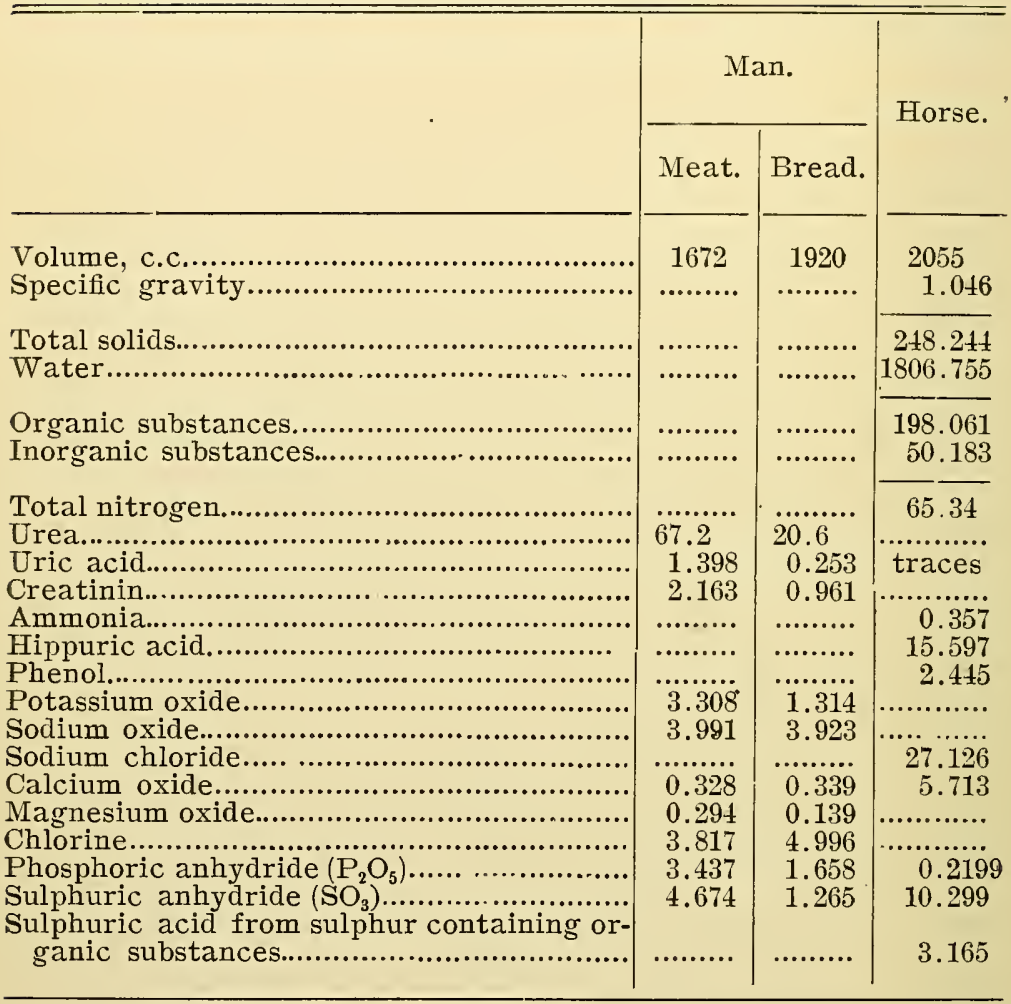

The results given in the first and second columns were obtained by Bunge from the urine of the same individual under exclusive meat and vegetable diet; each column represents the analysis of a single 24 hours' urine. The analytical results in the third column are those of E. Salkowski. 
In the following table are giren the arerages of the results obtained from the analysis of normal 24 hours' urine of students at the University of Michigan:

\begin{tabular}{|c|c|c|c|c|}
\hline \multirow[b]{2}{*}{ 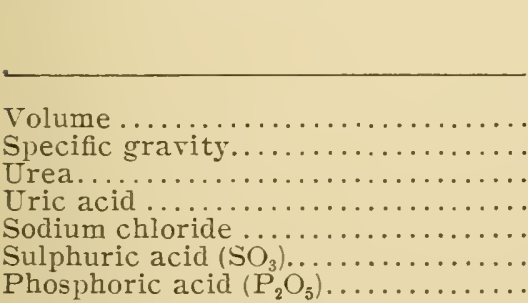 } & \multicolumn{2}{|c|}{ Nen. } & \multicolumn{2}{|c|}{ Women. } \\
\hline & $\begin{array}{r}1120 \\
1.022 \\
30.10 \\
.55 \\
13.93 \\
2.30 \\
2.16\end{array}$ & $\begin{array}{l}(333)^{*} \\
(334) \\
(320) \\
(266) \\
(275) \\
(266) \\
(308)\end{array}$ & $\begin{array}{c}1000 \\
1.020 \\
21.54 \\
.42 \\
11.19 \\
2.11 \\
1.78\end{array}$ & $\begin{array}{l}(56) \\
(56) \\
(43) \\
(36) \\
(42) \\
(40) \\
(44)\end{array}$ \\
\hline
\end{tabular}

* The figures in brackets refer to the number of determinations made.

\section{Milk Analysis.}

The milk to be analyzed should be thoroughly shaken just before each portion is taken for analysis, in order to insure a true sample. The quantity to be taken is measured out by means of a clean and dry 10 c.c. pipette graduated in $\frac{1}{10}$ c.c. The quantity of milk taken multiplied by the specific gravity gires the weight of the milk employed for the determination.

1.-Specific grarity.-Determine the specific gravity of the sample, at $15^{\circ}$, by means of :

(1). The picnometer, or specific gravity bottle. This is done in the following manner: The picnometer is cleaned, dried and weighed. It is then filled with distilled water at $15^{\circ}$ and weighed again: the difference is the weight of a certain rolume of water. The instrument is then dried, filled with the sample at $15^{\circ}$ and weighed again and the weight of the same volume of milk is thus obtained. The weight of the milk dirided by the weight of the same rolume of water gives the specific grarity.

(b). The lactometer. - There are two forms of this instrument in common use. The Querenne-Müller lactometer, employed largely in Europe, grives the specific gravity direct. The lactometer of the New York Board of Health reads from $0^{\circ}$, the density of water at $15^{\circ}$ and which corresponds to 1.0 , to $120^{\circ}$ which represents a specific 
gravity of $1.0348 .100^{\circ}$ on this scale represents the specific gravity of 1.029 , which is taken as the minimum density of genuine milk. In the absence of a lactometer the ordinary urinometer may be used, although the divisions are very small and the reading, consequently, is not accurate.

Place a sample of the milk in a suitable cylinder or $50 \mathrm{c.c}$. graduate and determine the density. Compare the reading thus obtained with the speciflc gravity as given by an ordinary lactometer.

Determine the specific gravity of the skimmed milk obtained from the following experiment. What is the result?

What is the effect of the addition of water to milk? To skimmed milk? What is the effect of the removal of cream?

2.-Creamometer.-Fill a 50 c.c. graduate to the mark with milk, cork and set aside for 24 hours at the ordinary room temperature. Note the volume of the cream and calculate the volume per cent. A good milk should give 10-12 per cent. of cream.

Remove the skimmed milk from below the layer of cream by means of a pipette and determine the density according to $1 b$.

3.-Total solids.--Place 2 c.c. of milk in a previously weighed watch-glass and evaporate on the water-bath to dryness. Then wipe the bottom of the watch-glass and place it in an air-bath at $100-105^{\circ}$ for 3 hours. Cool in the desiccator and weigh rapidly. Calculate the per cent. of total solids. This result subtracted from 100 gives the per cent. of water.

t.-Ash.-Place 5 c.c. of milk in a previously weighed porcelain crucible and evaporate to dryness on the water-bath. Then carefully ignite so as to char the mass slowly and thus avoid spurting. The ignition must be continued till the ash is grayish-white and free from carbon. Cool in the desiccator and weigh. Calculate the per cent. of ash.

5.-Fat.-Roll up into a coil a strip of thick filter paper about 2 inches wide and 24 inches long, and tie it with a thread or wire. Five c.c. of milk are allowed to run slowly onto one end of the coil. The coil, dry end down, is placed on a watch-glass and dried in an air-bath at $100-105^{\circ}$ for one hour, or longer if necessary. It is then placed in a Soxhlet extraction apparatus which is connected, by means of sound, well fitting corks, to an inverted condenser above, and to a $\mathbf{1 5 0}$ c.c. wide neck, round, or Erlenmeyer flask below (Fig. 8). The weight of the clean, dry flask is first ascertained. By means of a small fun nel pour ether into the apparatus from above, until it siphons: then 
add about half as much more ether. The flask is now heated, cautiously, on a water-bath so that the ether will siphon about every five minutes. The extraction will be completed in from 1 to $1 \frac{1}{2}$ hours. Then remore the paper coil and continue heating till the ether fills the extraction apparatus, and is almost ready to siphon. Disconnect the flask and transfer the ether from the apparatus to a bottle. The flask still contains some ether. Heat on the water-bath to dryness, wipe carefully, and finally dry in an air-bath at $100-105^{\circ}$ for one hour. Cool in a desiccator or in the balance case and weigh. Calculate the per cent. of fat. Subtract this result from that of total solids; the difference is solirls not fut.

In working with ether great care must be taken to prevent accidents. The corks must be large enough to fit snug-

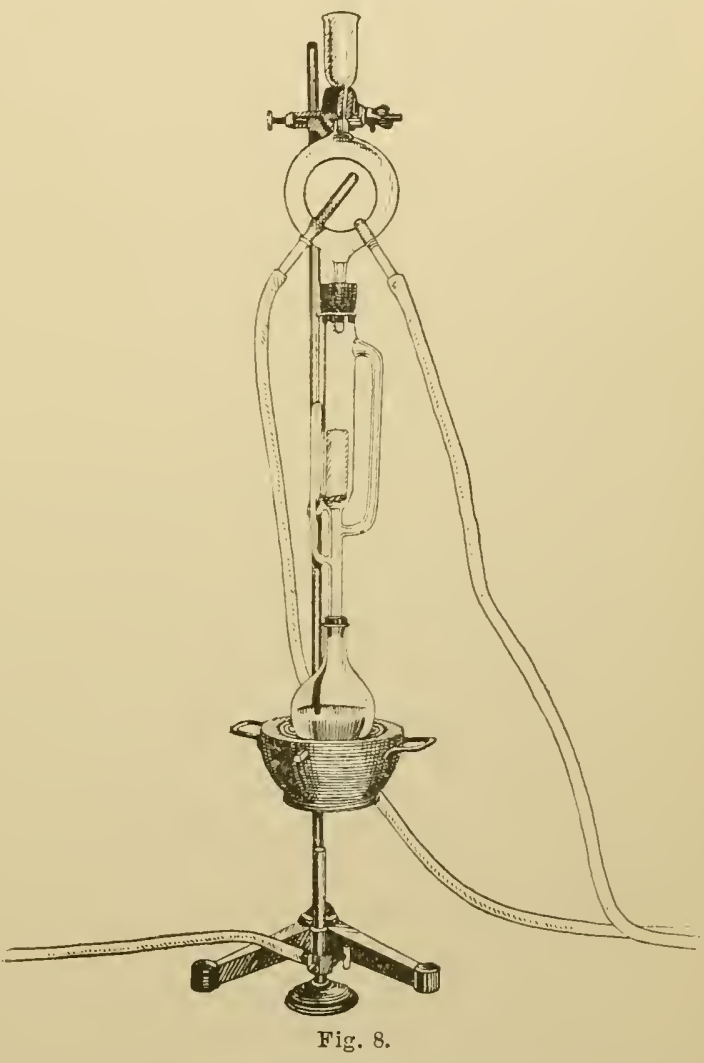
ly, and the gas must be turned off until everything is in readiness to begin the extraction. To obtain very accurate results the coil of paper should be first extracted with ether to remove what fat may be present. Paper, from which fat has been removed, can be purchased.

6.-Lrectose.-Place about 380 c.c. of water in a beaker, add 20 c.c. of milk and mix thoroughly. Then add gradually about 2 c.c. of dilute acetic acid (1: 10), with constant stirring, till a flocculent precipitate forms. The reaction should be distinctly acid. Place the beaker on a wire gauze and heat to hoiling for $1 / 2$ hour; then filter through a 
wet filter. Rinse the beaker several times with hot water, and finally wash the residue on the filter, proteids and fat, with hot water. Concentrate the combined filtrate and wash-water to about 150 c.c. Cool and dilute in a 200 c.c. measuring flask to the mark. Determine the lactose in this solution with Fehling's solution according to the method described on p. 247.

10 c.c. of Fehling's solution corresponds to $0.067 \mathrm{~g}$. of milk sugar. Calculate the per cent. of lactose.

7.-Casein.-To 50 c.c. of water in a small beaker add 10 c.c. of milk and mix. Warm on the water-bath to $40^{\circ}$, then add $21 / 2$ c.c. of potassium alum solution (1:10) and stir thoroughly. A finely flocculent precipitate forms which should settle rapidly and the liquid should be clear. Let stand for about 15 minutes at $40^{\circ}$, then filter. If the filtrate is cloudy pass it again through the filter. Wash several times with water. Reserve the combined filtrate and washwater for the next determination.

Place the filter with its contents in a Kjeldahl flask of about 250 c.c. capacity, and treat exactly according to the method given for total nitrogen in urine, p. 258.

A blank experiment with a filter paper and all the reagents should be carried through in exactly the same manner in order to ascertain how much $\mathrm{NH}_{3}$ may be given off by the reagents themselves. The number of c.c. of $\frac{\mathrm{N}}{10} \mathrm{NH}_{3}$ thus found should be subtracted as a correction from the total number of c.c. of $\frac{\mathrm{N}}{10} \mathrm{NH}_{3}$ given off in the determination. The difference multiplied by the $\frac{N}{10}$ factor of nitrogen, 0.0014, gives the amount of nitrogen contained in the casein precipitated from 10 c.c. of milk. This amount of nitrogen multiplied by 6.37 gives the amount of casein in 10 c.c. of milk. Calculate the per cent. of casein.

8.-Globulin and albumin.-The filtrate from the alum precipitate of casein in the preceding experiment contains globulin and albumin. To this filtrate add 10 c.c. of Almén's tannic acid solution. Filter off the voluminous precipitate of proteids, wash several times with water, and allow to drain. Place the filter and contents in a Kjeldahl flask and determine the nitrogen as above, making the proper correction for a blank. The amount of nitrogen found multiplied by the factor 6.37 gives the amount of albumin and globulin in 10 c.c. of milk. Calculate the per cent. of albumin and globulin.

Almén's tannic acid solution is prepared according to the following formula: $4 \mathrm{~g}$. of tannic acid; 8 c.c. of 25 per cent. acetic acid; 90 c.c. of 90 per cent. alcohol; 100 c.c. of water. 
9.-Total nitrogen in milk.-Place 10 c.c. of milk in a Kjeldahl flask, add 15 c.c. of $\mathrm{H}_{2} \mathrm{SO}_{4}$ and treat as abore under Exp. 7. This gives the total nitrogen present in the milk and serres as a control on the two preceding determinations.

A report of the results obtained is to be made out, together with a statement as to whether the milk is adulterated or not:

1. Specific grarity, whole milk.

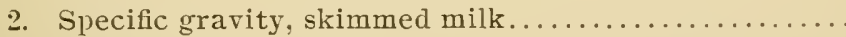

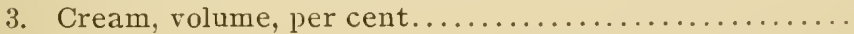

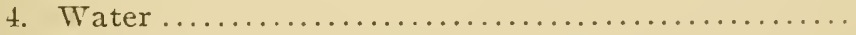

5. Total solids .

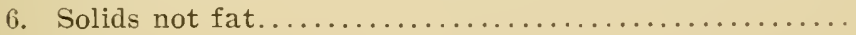

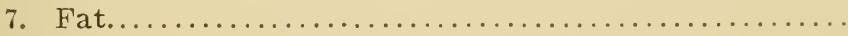

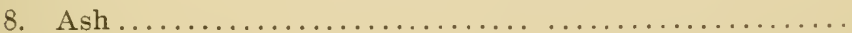

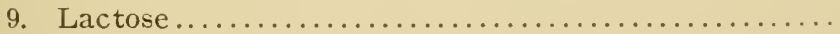

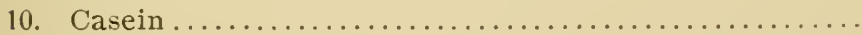

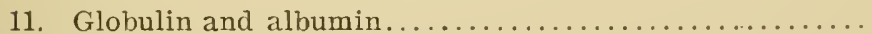

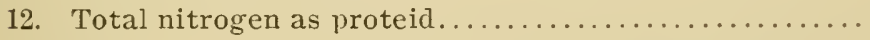

To decide upon the purity of a milk the determinations giren under 1,5 , and 7 ( 4 and 6 by difference), are as a rule sufficient. In case of doubt the ash may be determined. The legal standard of milik varies in different states. In New York the minimum of total solids allowed is 12 per cent.: of fat 3 per cent. In Massachusetts the total solids must not fall below 13 per cent. New Jersey allows a minimum of 12 per cent. for total solids.

The following table, compiled from König, shows the arerage percentage composition of various milks:

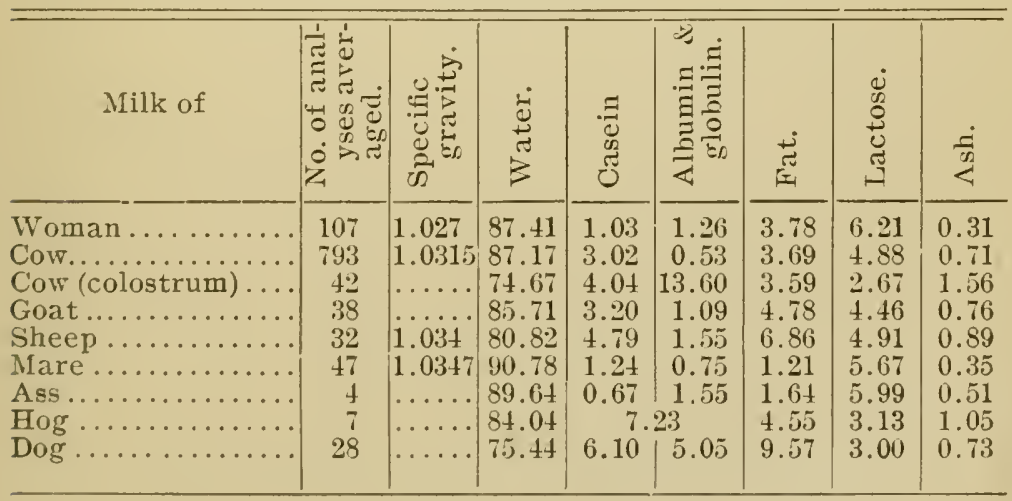




\section{Quantitative Analysis of Gastric Juice.}

Toepfer"s method.-The following reagents are necessary:

1.-Deci-normal sodium hydrate solution.

2.-A 1 per cent. alcoholic solution of phenol-phthaleïn. This indicates total acidity.

3.-A 1 per cent. aqueous solution of sodium alizarin-sulphonic acid. This indicates all acids except the loosely combined hydrochloric.

4.-A 0.5 per cent. alcoholic solution of di-methyl amido-azobenzol. This indicates only free $\mathrm{HCl}$.

The method is as follows: Measure out into each of three beakers 10 c.c. of the filtered gastric juice. If necessary a smaller amount may be taken, or the gastric juice may be diluted.

To beaker No. 1 add 1-2 drops of phenol-phthaleïn, then run in the deci-normal sodium hydrate, not to the first pink color, but to a dark red which no longer increases in intensity. Note the number of c.c. of reagent employed.

To beaker No. 2 add 3-4 drops of the alizarin solution and then titrate with deci-normal sodium hydrate till the first pure violet color is reached. Note the number of c.c. employed.

To beaker No. 3 add $3-4$ drops of di-methyl amido-azobenzol solution. A yellow color indicates the absence of free $\mathrm{HCl}$. If a red color is present run in deci-normal sodium hydrate till it just disappears. Note the number of c.c. employed.

The number of c.c. required for beaker No. 1 gives the total acidity.

The difference between the number of c.c. of deci-normal sodium hydrate required for beaker No. 1 (total acids) and the number required for beaker No. 2 (all acids except loosely combined), gives the loosely combined $\mathrm{HCl}$.

The number of c.c. required for beaker No. 3 gives the free $\mathrm{HCl}$.

The number of c.c. of reagent required for beaker No. 2 minus the number of c.c. required for beaker No. 3, gives the organic acid and acid salts.

The student will receive two unknowns, containing free and loosely combined hydrochloric acid and an organic acid. In reporting 
the results, give the number of c.c. of $\frac{N}{10}$ alkali that would be required to neutralize each of the acids in 100 c.c..

\section{Blood.}

The examination of blood is of very great clinical importance and every student should make himself familiar with the methods that are usually employed. The examination from the physical and chemical standpoint embraces a determination of the specific gravity; counting of red and of white blood cells; and a determination of the amount of hæmoglobin present.

1.- * Specific gravity. - In the case of blood from a patient it is not possible to determine the density according to the methods described under urine and milk. A much smaller amount must suffice. If a drop of blood is introduced into a mixture of chloroform and benzol it will not mix but will either float on the surface, or sink to the bottom, or remain in the body of the liquid where it is placed. If the drop floats it is because the liquid is heavier and in that case benzol is to be added, a few drops at a time and mixed by means of a rod, till the drop remains wherever it is placed. If on the other hand the drop falls to the bottom it shows that the liquid is too light and chloroform is then to be added in portions as in the case of benzol, till the drop remains stationary wherever placed. In the latter case the drop of blood has the same specific gravity as the liquid in which it floats. The specific gravity of the mixture is therefore taken and this represents the density of the blood. The ordinary urinometer may often be used, if graduated to about 1.050 , when the specific gravity is low. It is better to have one that will read from 1.040 to 1.070 . The measuring cylinder which receives the mixture should be abso. lutely clean and dry. Several separate drops of blood may be advantageously employed. The mixture of chloroform 
and benzol should at the beginning have a density of about 1.059 , which is that of normal blood.

Inasmuch as the specific gravity of blood is subject to variation, not so much because of differences in the density of the plasma, but because of differences in the number of cells and hence the amount of hæmoglobin present, it follows that this method can also be used to determine approximately the amount of hæmoglobin present.

2.- Determination of hcemoglobin. - This can be determined very accurately by means of Hüfner's spectrophotometer, but this instrument is not used for clinical purposes. Among the clinical methods may be mentioned the following:

(a). From specific gravity of blood.-From tables especially prepared the amount of hæmoglobin present can be readily ascertained. A specific gravity of 1.059 (normal blood) represents 100 per cent. of hæmoglobin. Hammerschlag's tables, as given in Cabot's "Examination of Blood," can be used for this purpose. The method is not applicable in cases of dropsy.

(b). Fleischl's hcemometer.-In this method a minute, definite quantity of blood is transferred to water and the color of this solution is compared with that of a wedge of colored glass. When the colors match the per cent. of hæmoglobin is read off on the scale; 100 corresponds to the color of the normal blood of man, about 90 corresponds to that of woman. This instrument, although it gives only approximate results, is the one most commonly employed.

(c). Hoppe-Seyler's colorimetric method.-In this method the blood is treated with carbon monoxide and the color of the resulting carbon monoxide hæmoglobin solution is compared with a solution of pure carbon monoxide hæmoglobin of known strength. The two colors can therefore be matched perfectly, which is not always the case in the 
preceding method. Still greater accuracy is attained by eliminating the partition that exists between the two compartments by means of an Albrecht glass cube. In that case the colors are compared side by side as in a SoleilVentzke saccharimeter. Although the apparatus is some-

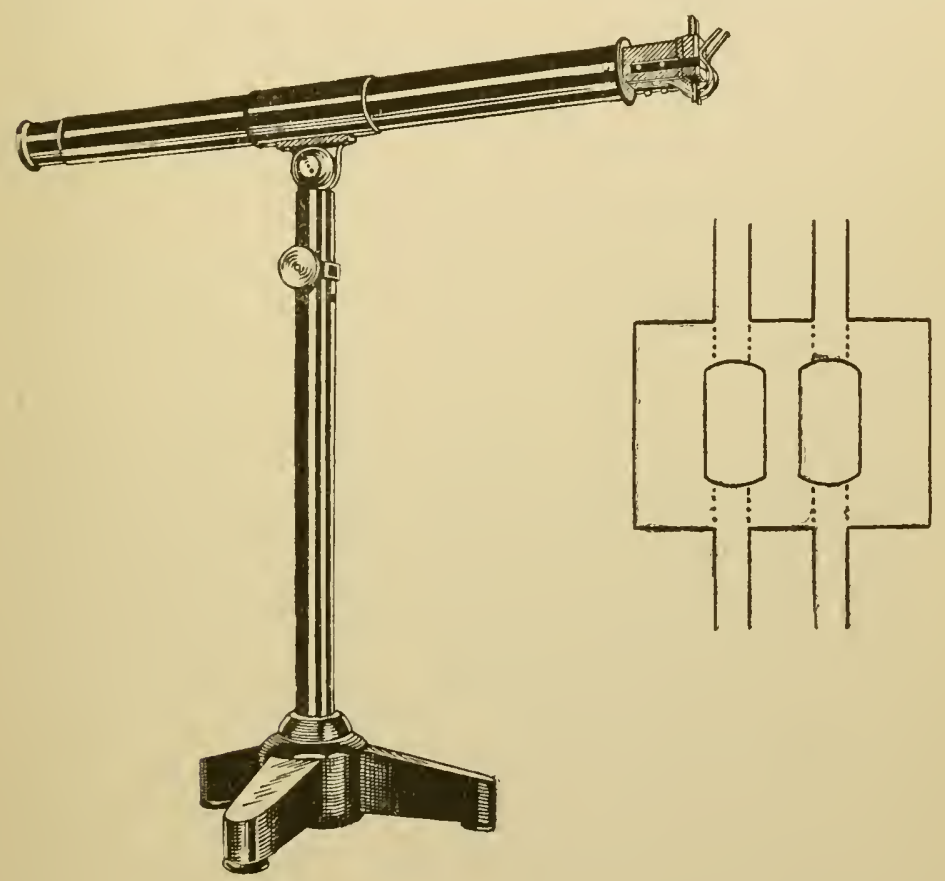

Fig. 9.

what expensive it can be easily manipulated and gives much more accurate results than either of the preceding (Fig. 9).

Recrystallized hæmoglobin from the dog, or horse is employed. A strong solution (2-3 per cent.) of this hæmoglobin is made and treated with carbon monoxide. In a portion of this solution the amount of hæmoglobin present is determined by evaporating, drying and weighing. The remainder of the solution, saturated with carbon monoxide, is drawn up, in portions of about 6 c.c., into glass tubes. 
and these are then sealed at both ends. These solutions once prepared will keep perfectly. Before use the contents of one of the tubes is diluted with water treated with carbon monoxide till the solution contains 0.2 per cent. of CO-hæmoglobin.

The blood, obtained by puncture, is drawn up into a graduated capillary pipette such as is used in the ThomaZeiss blood counter. 0.04 to 0.06 c.c. or more of the blood is measured out and transferred to a small graduated cylinder (10 c.c. graduated in 0.1 c.c., stoppered). The pipette is carefully rinsed several times with distilled water and this is added to the cylinder. A drop of very dilute $\mathrm{NaOH}$ solution is added and then a current of illuminating gas is passed through the liquid to convert the pigment into $\mathrm{CO}$ hæmoglobin.

The standard 0.2 per cent. CO-hæmoglobin solution is placed in a similar cylinder and the colors are compared. The blood tube will have a deeper color, hence water saturated with carbon monoxide is added till the two solutions have approximately the same color. They are then drawn up into the "double pipette" and compared. Further addition of water is made to the blood till on comparison it has the same color as the standard 0.2 per cent. CO-hæmoglobin solution. Now, note the volume to which the blood has been diluted to obtain this color. Knowing the amount of blood taken the per cent. of hæmoglobin can be readily calculated from the formula:

$$
\text { Per cent. of hæmoglobin }=\frac{0.002 \times v \cdot \times 100}{w}
$$

$w$ represents the weight (or volume) of the blood taken; $v$ represents the volume to which this was diluted; 0.002 represents the contents of the standard CO-hæmoglobin solution. By this method the blood of normal individuals contains on an average 14.08 per cent. of hæmoglobin. The results are higher than those obtained by Fleischl's 
hæmometer or Gower's hæmoglobinometer. The above description of Hoppe-Seyler's "double-pipette" is given at length because this valuable instrument has not received the attention that it undoubtedly merits.

3.- * Counting of corpuscles. - The Thoma-Zeiss bloodcounter is commonly employed for this purpose. An excellent description of the use of this instrument will be found in Cabot's work on "Blood".

Each student will receive 25 quantitative unknowns, containing the substances indicated below. These are to be determined, and a report made on blanks provided for that purpose.

Nos. 1 and 2 contain oxalic acid.

Nos. 3 and $t$ contain $\mathrm{NaOH}$.

Nos. 5 and 6 contain $\mathrm{NaCl}$ (use Volhard's method).

Nos. 7 and 8 contain $\mathrm{NaCl}$ (use Mohr's method).

Nos. 9 and 10 contain $\mathrm{SO}_{3}$ (volumetrically).

Nos. 11 and 12 contain $\mathrm{P}_{2} \mathrm{O}_{5}$ (volumetrically).

Nos. 13,14 and 15 contain glucose.

No. 16 contains $\mathrm{NaCl}$, or $\mathrm{SO}_{3}$, or $\mathrm{P}_{2} \mathrm{O}_{5}$ (gravimetrically).

Nos. 17 and 18 contain urea (use Liebig's method).

Nos. 19 and 20 contain urea (use ureometer).

No. 21 contains uric acid (use Heintz' method).

No. 22 contains uric acid (use Folin's or Ludwig's method).

No. 23 contains albumin (gravimetric method).

Nos. 24 and 25 contain free and loosely combined $\mathrm{HCl}$, and organic acids in gastric juice (Toepfer's method).

Each student will also make a complete quantitative analysis of 24 hours' urine, and make a report on the same. 


\section{Laboratory of Physiological Chemistry.}

REPORT ON QUANTITATIVE UNKNOWNS.

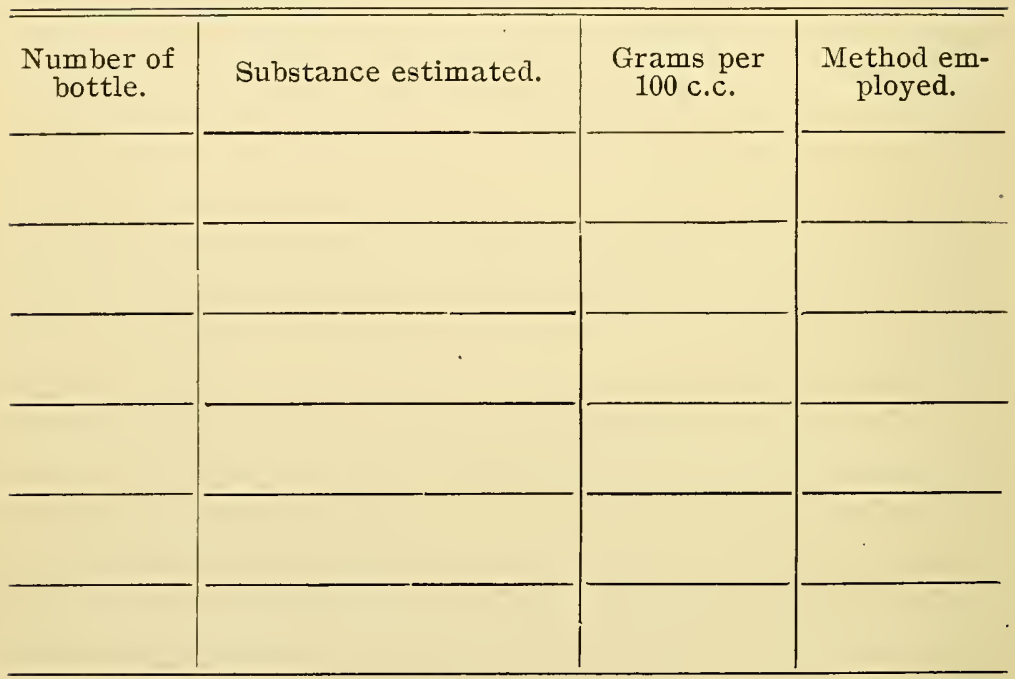

Dated................. 189. 


\section{Laboratory of Physiological Chemistry.}

\section{REPORT ON EXAMINATION OF URINE.}

Sample from.

Submitted by

Receired

189..; Reported.

189

PHTSICAL AND CHEMICAL EXAMINATION.

Total quantity for $2+$ hours.........

Specific gravity............. Total solids

Reaction .....................; Equivalent to.............

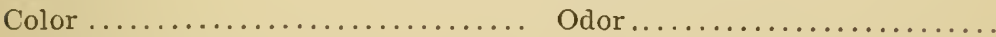

General appearance...............

Sediment, color................ quantity ...

\begin{tabular}{|c|c|c|c|c|}
\hline & 总 & 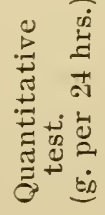 & & 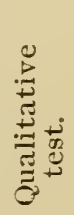 \\
\hline 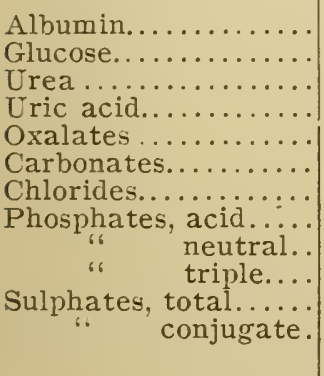 & & & 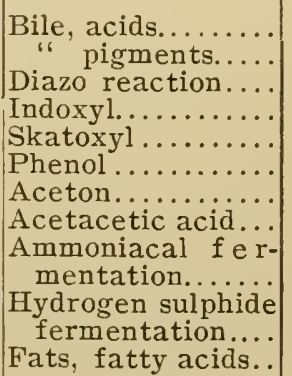 & \\
\hline
\end{tabular}

MICROSCOPIC EXAMINATION.

Unoiganized sediment.

Crystalline

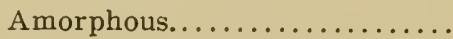

Organized sediment.

Epithelial cells from

Non-pathogenic bacteria.

Casts.

Pathogenic bacteria..........

Blood

Yeasts

Pus..

Moulds

Spermatozoa

Animal parasites 


\section{Final Unknowns.}

Each student will receive 25 urine "unknowns." These are not pathological urines, but they do contain one or more of the common abnormal constituents of urine and are given to the student as a matter of exercise to review and apply the characteristic tests employed in their recognition.

The first 15 unknowns may contain one or more of the following constituents:

$\begin{array}{ll}\text { Sugar, } & \text { Uric acid, } \\ \text { Albumin, } & \text { Urates, } \\ \text { Bile, } & \text { Calcium oxalate, } \\ \text { Blood, } & \text { Amorphous phosphates, } \\ \text { Hæmoglobin, } & \text { Triple phosphate, } \\ \text { Fats, } & \text { Calcium acid phosphate, } \\ \text { Pus, } & \text { Calcium carbonate. }\end{array}$

Casts.

The next five may contain in addition to the above constituents:
Hippuric acid,
Albumose,
Diazo reaction,
Pyrocatechin,
Indican,
Hydrogen sulphide.

The last five may contain in addition to one or more of the preceding:
Urea, excess or deficiency,
Leucin,
Aceton, Tyrosin,
Acetacetic acid,
Cholesterin.

The results obtained on examination of these unknowns are reported, in sets of five, on blanks provided for that purpose. 


\section{Laboratory of Physiological Chemistry.}

REPORT ON UNKNOWNS.

No

Reaction

Sp. Gr.

Soluble constituents

Sediment

No.

Reaction

Sp. Gr.

Soluble constituents

Sediment

No.

Reaction

Sp. Gr.

Soluble constituents.

Sediment

No.

Reaction

Sp. Gr

Soluble constituents.

Sediment

No.

Reaction

Sp. Gr

Soluble constituents.

Sediment

Dated.

Name 



\section{CHAPTEP XII.}

\section{TABLES FOR EXAMINATION OF URINE."}

Collect the urine passed during $2 \pm$ hours. mis and measure. A-certain the specific gravity and reaction: note the color. odoz. and general appearance, whether cloudy or clear. Set a portion aside in a clean glass ressel better, a conical one) and allom the deposit to subside for some hour for microscopic examination. as giten in the following tables (A), B) and (C). Considerable time can be sared oy the use of a centrifuge. Filter another portion and test the clear filtrate according to table (D.

\section{MICROSCOPIC EKAMTAIION OF LRIAAPT DEPOSITS.}

After the deposit has formed. or has been thrown out oj centrifugation transfer by means of a small pipette a drop from the bottom of the ressel. place this on a glass slide. corer with a corerslip and examine under a microscope which magnifes from $300 \mathrm{j}$ to 500 diameters. It is best to examine frst with a lom power $\left(z_{3}\right.$ inch objectire) then with a higher power (1/5 inch). For pathogenic organisms a $\frac{1}{12}$ inch homogeneous oil-immersion ojjective is neceseary. The objects seen under the microscope may be either crystalline amorphous. or organized: any one. or all three of these groups maj be represented. The same substance may appear at one time in crystals. and at another in the amorphous form. and may thus indicate different pathological conditions. In applying reagents to the sediment on a slide aroid learing any of the liquid on top of the coter glass. A glass tube drawn out in the middle and cut in two mill furnish useful pipettes. In order to cause the reagent to go under the corer-glass a piece of blotting or flter paper mag be apolied to the opposite edge of the corer-glass.

The followirg diectiors and tables, with slizht alte:atioss, are tsiser frou

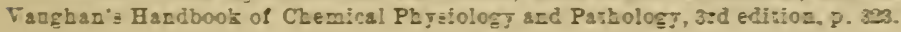




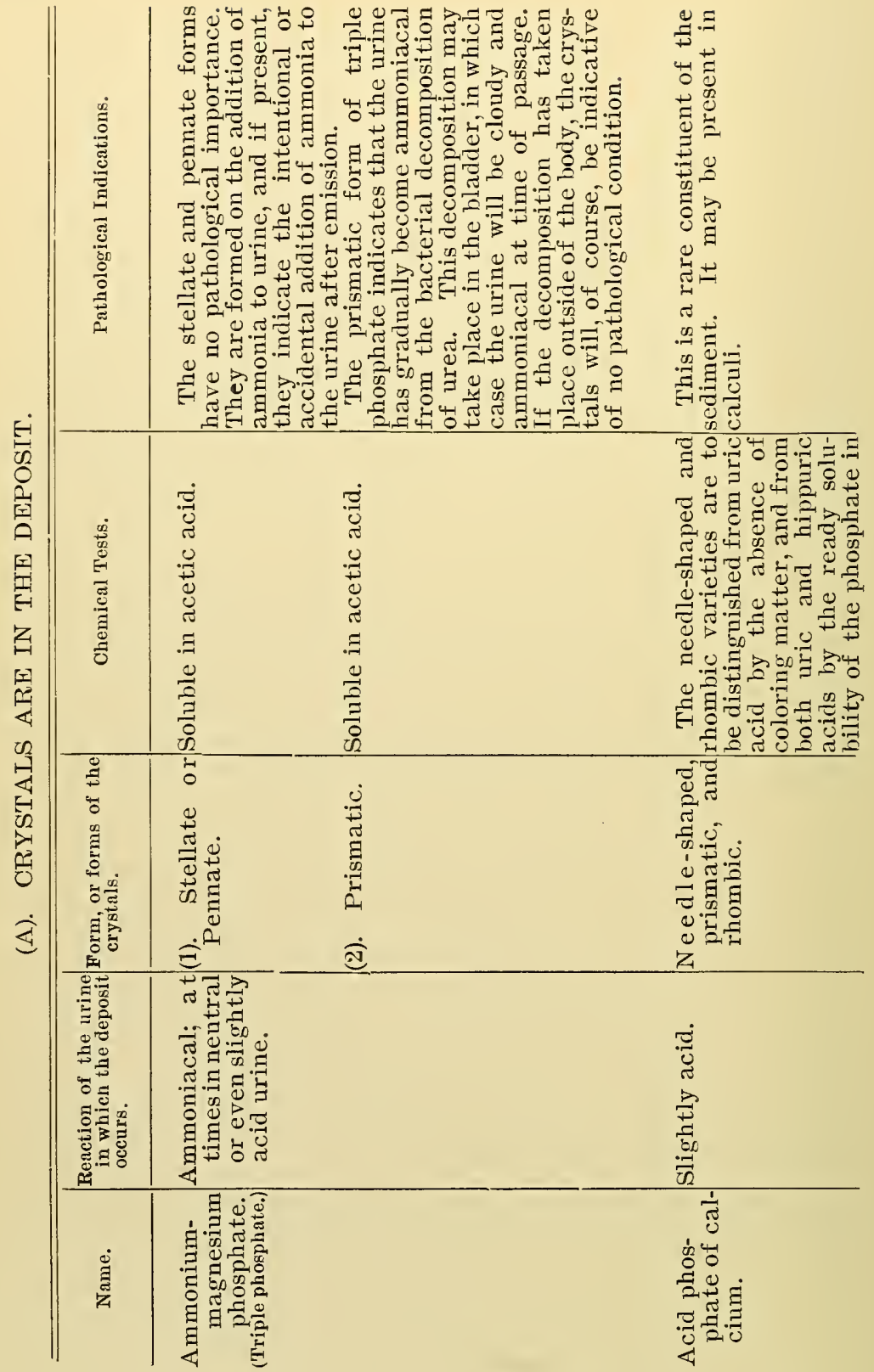




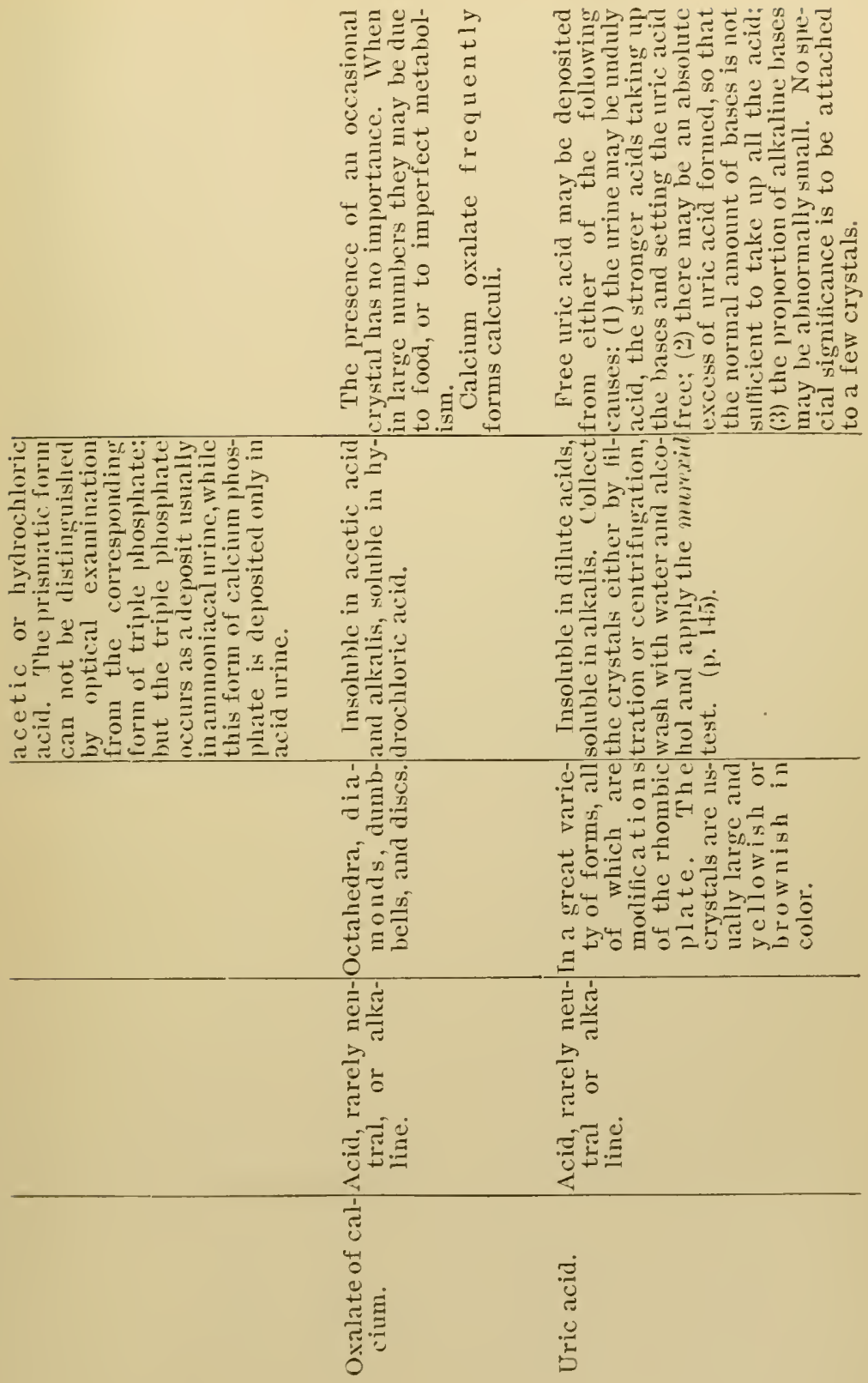




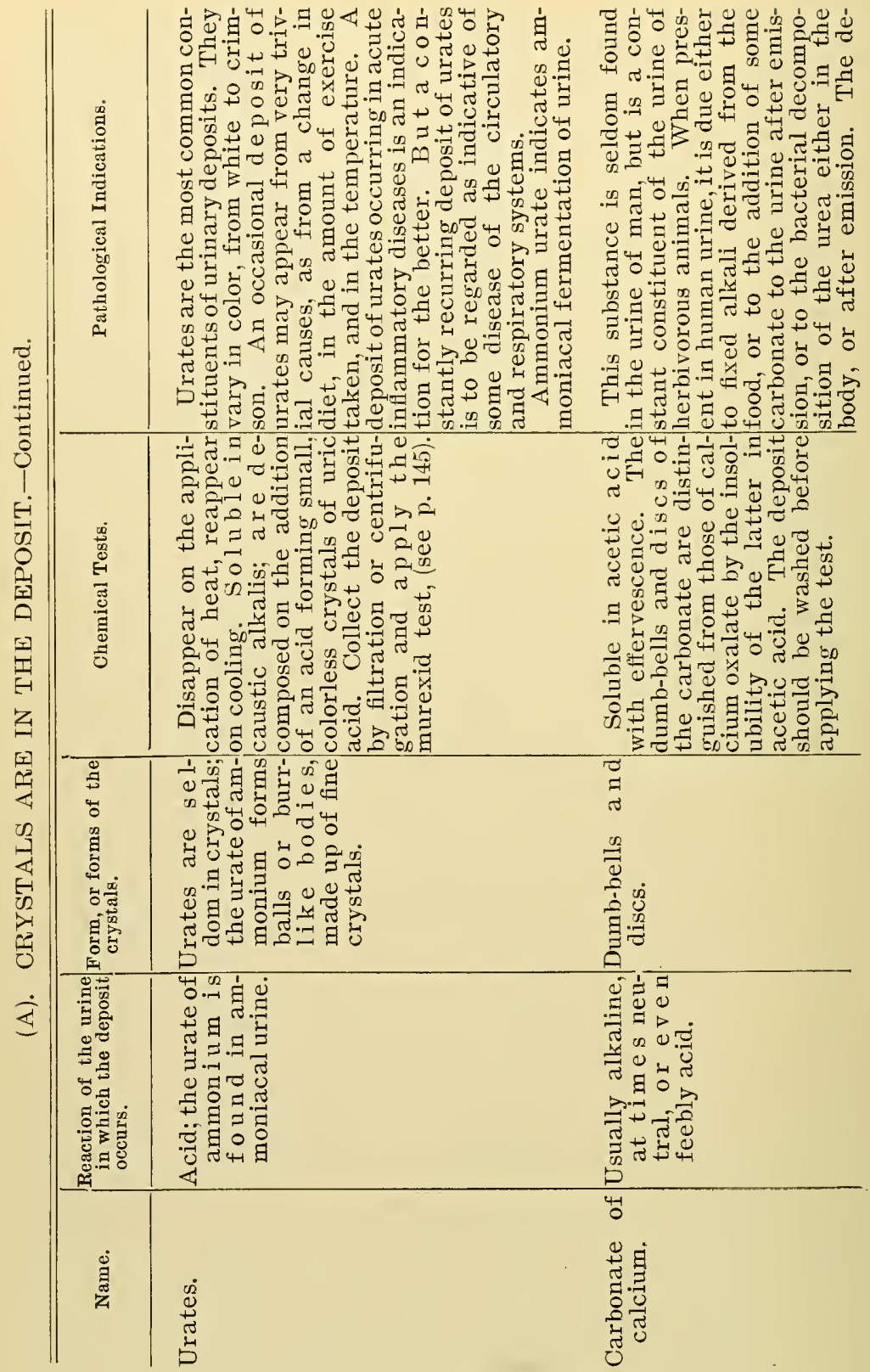



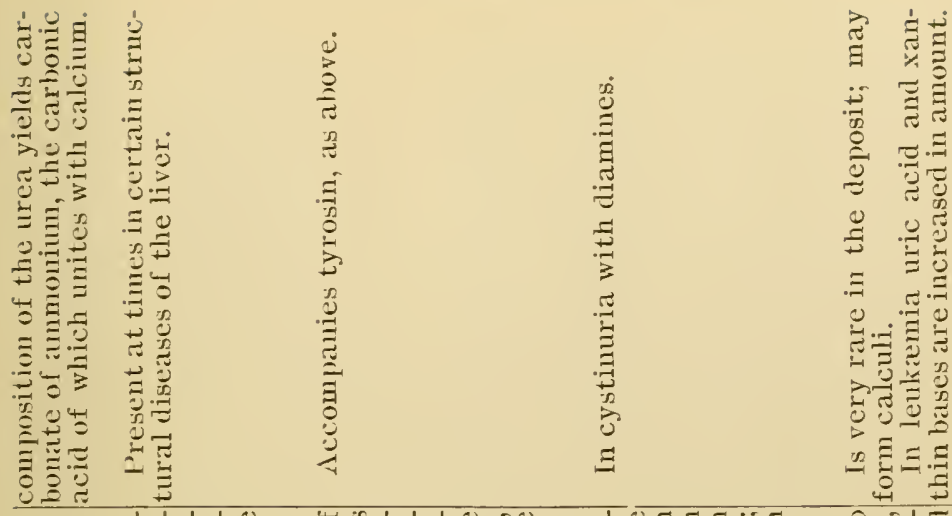

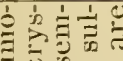

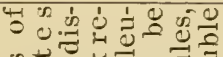

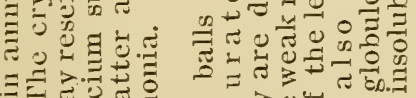

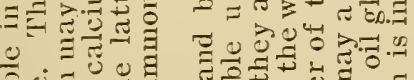

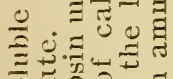

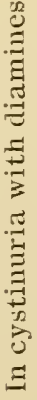

表完

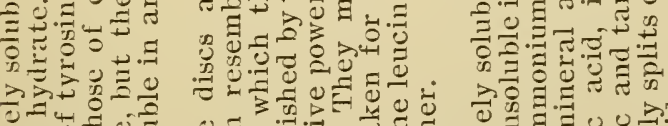

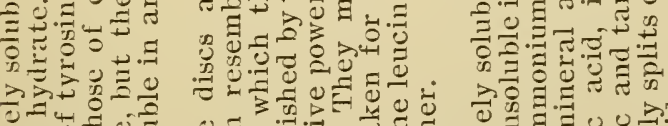

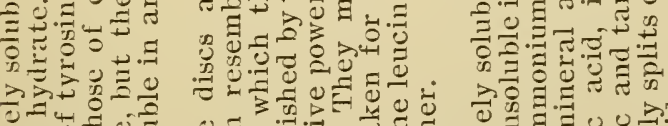

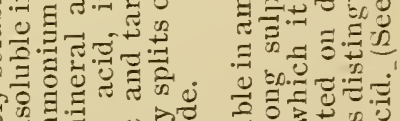
ङ

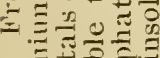
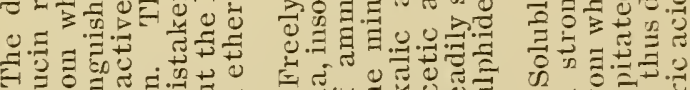

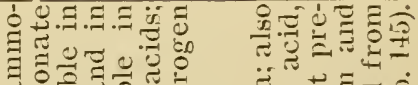
조을 $\exists$ 궁

(1)

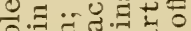

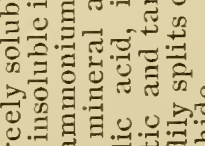
‥

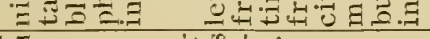
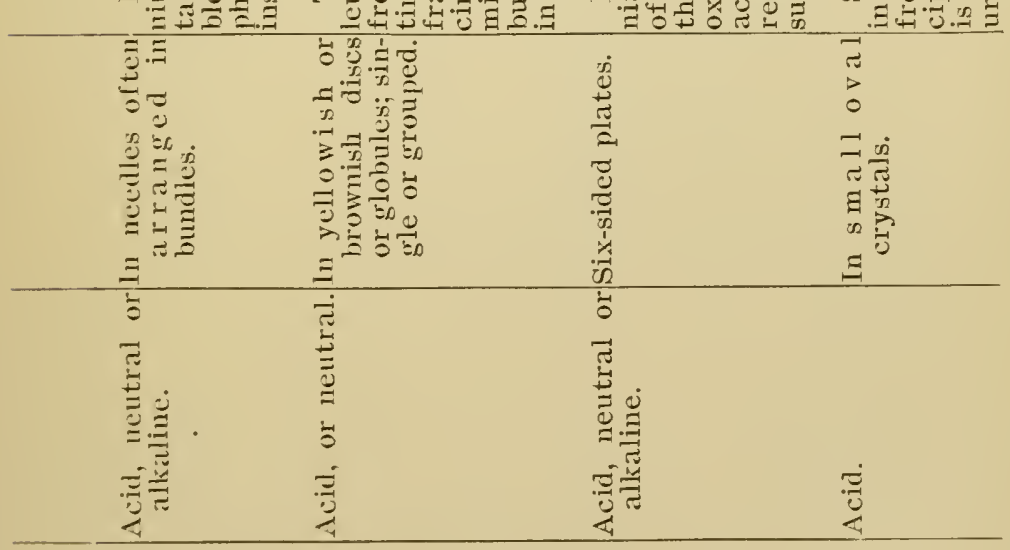

$\exists:=0$

$\frac{1}{2}$

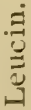

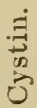

ב⿱艹 


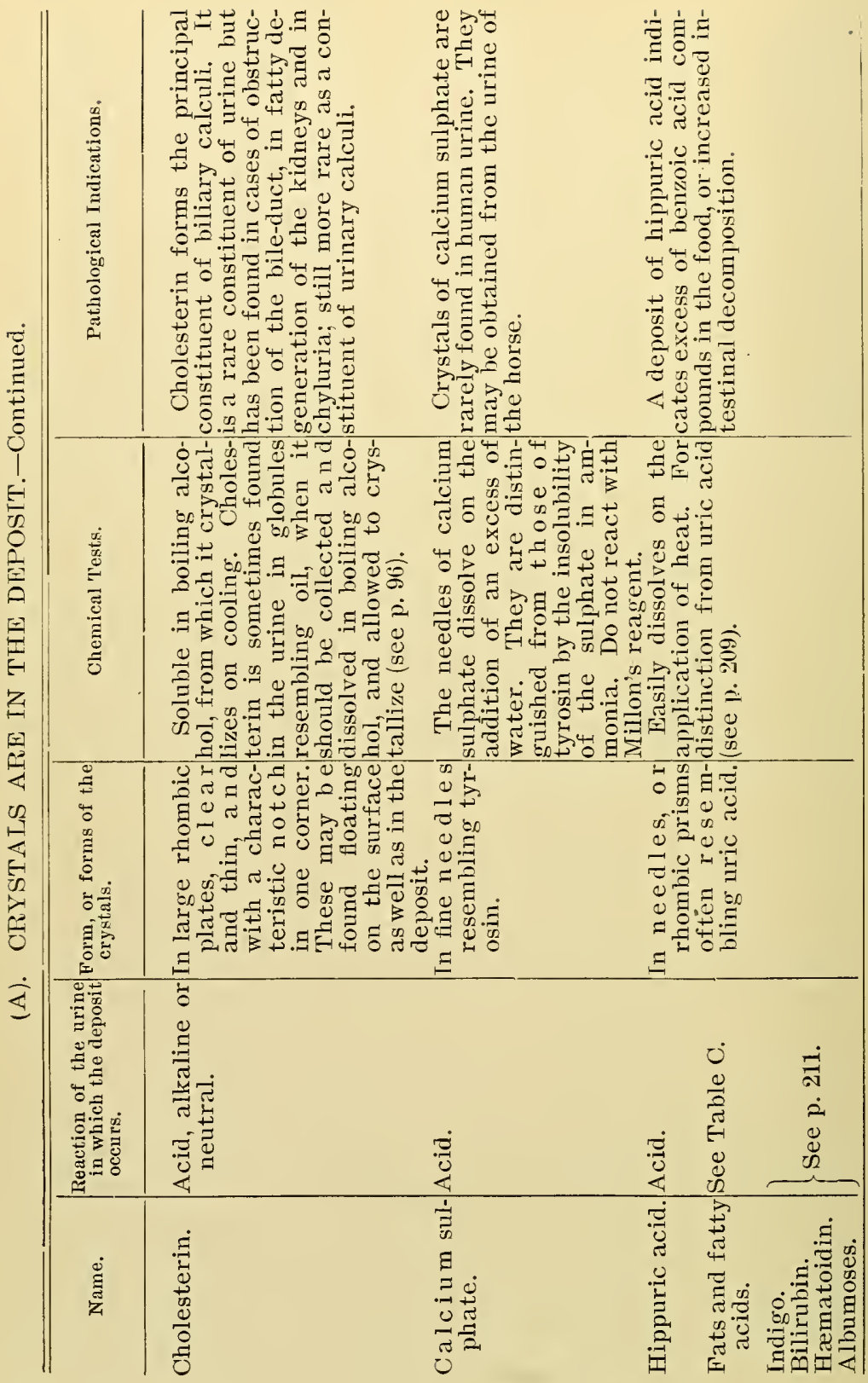




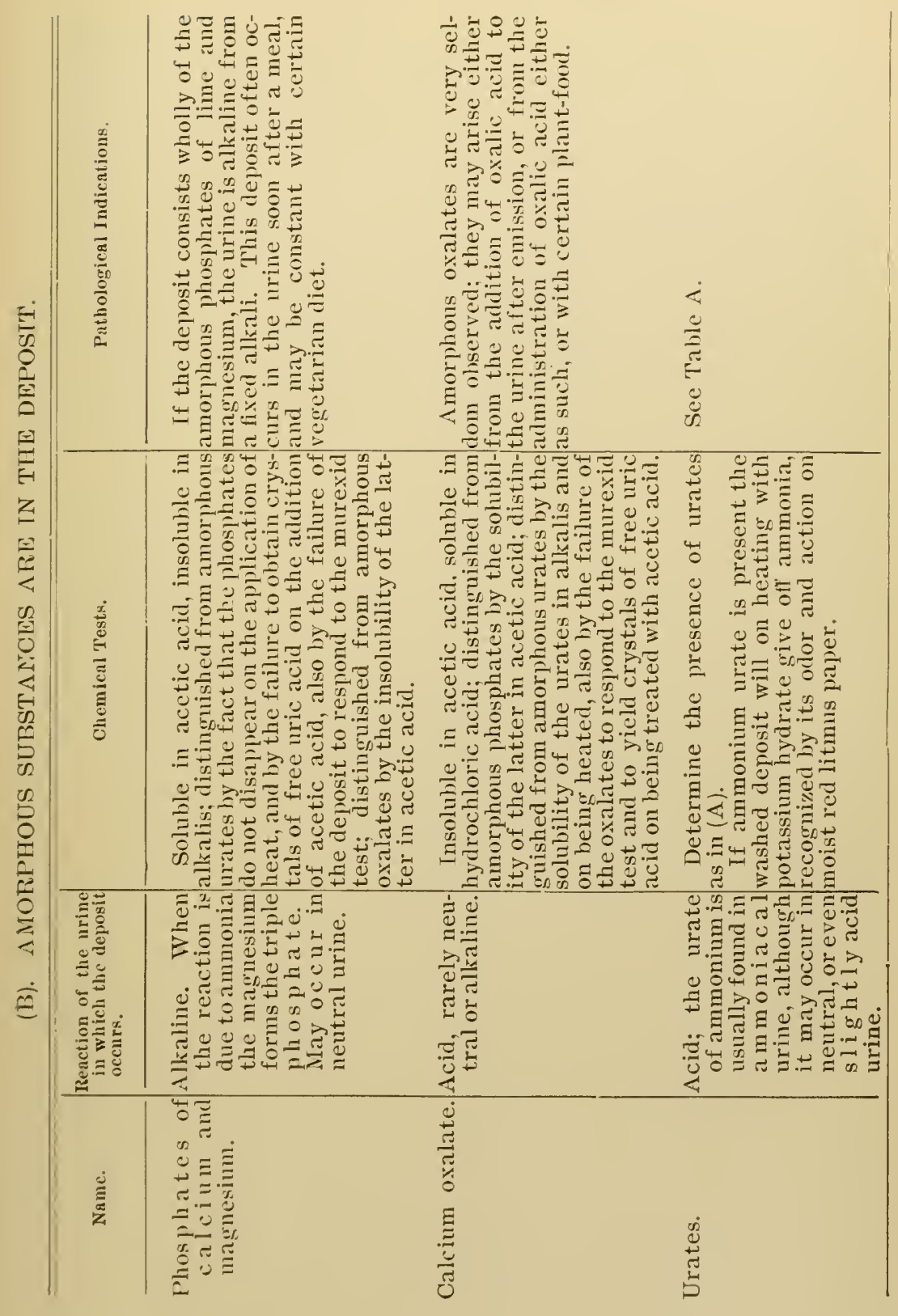




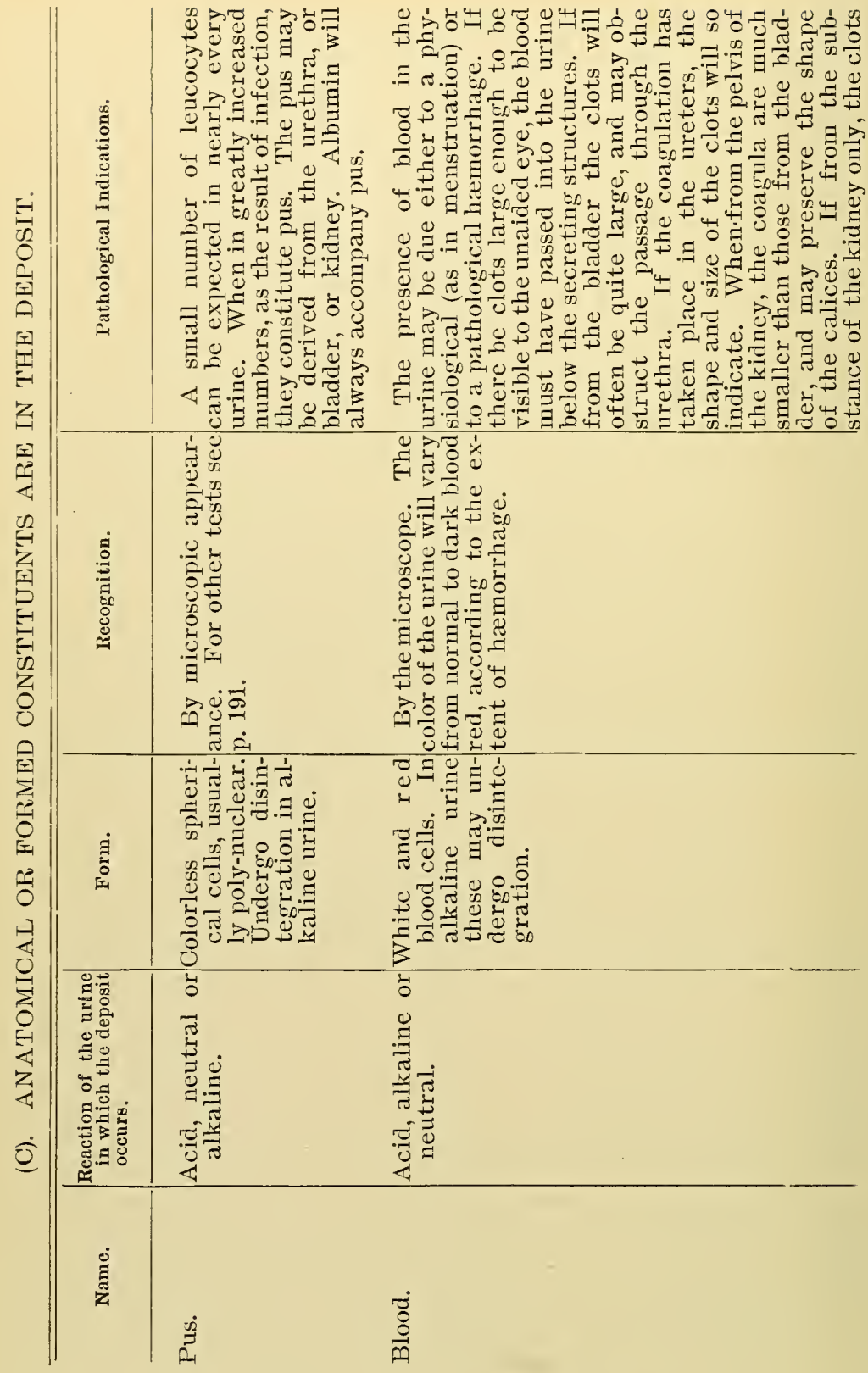



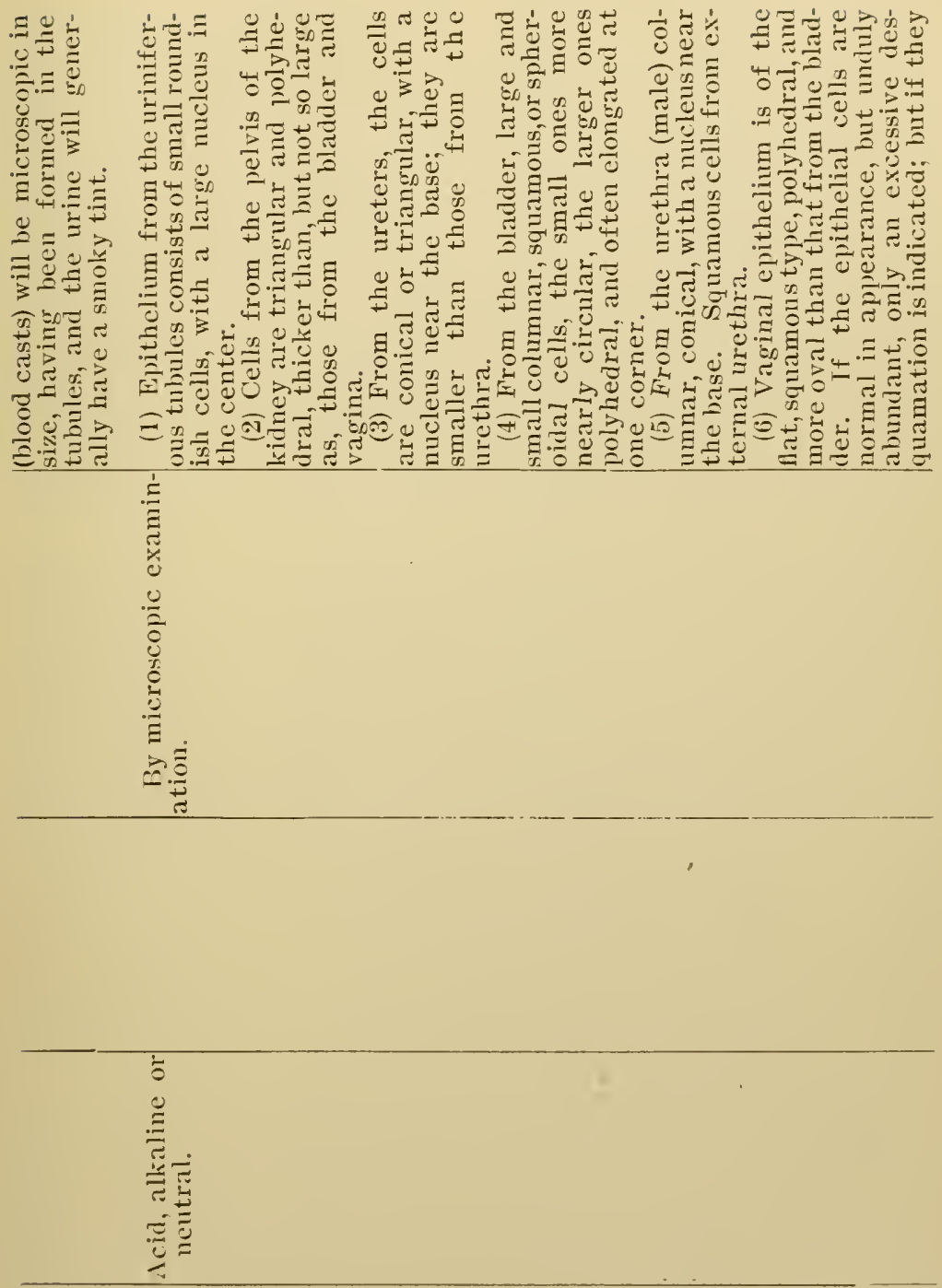

咅 


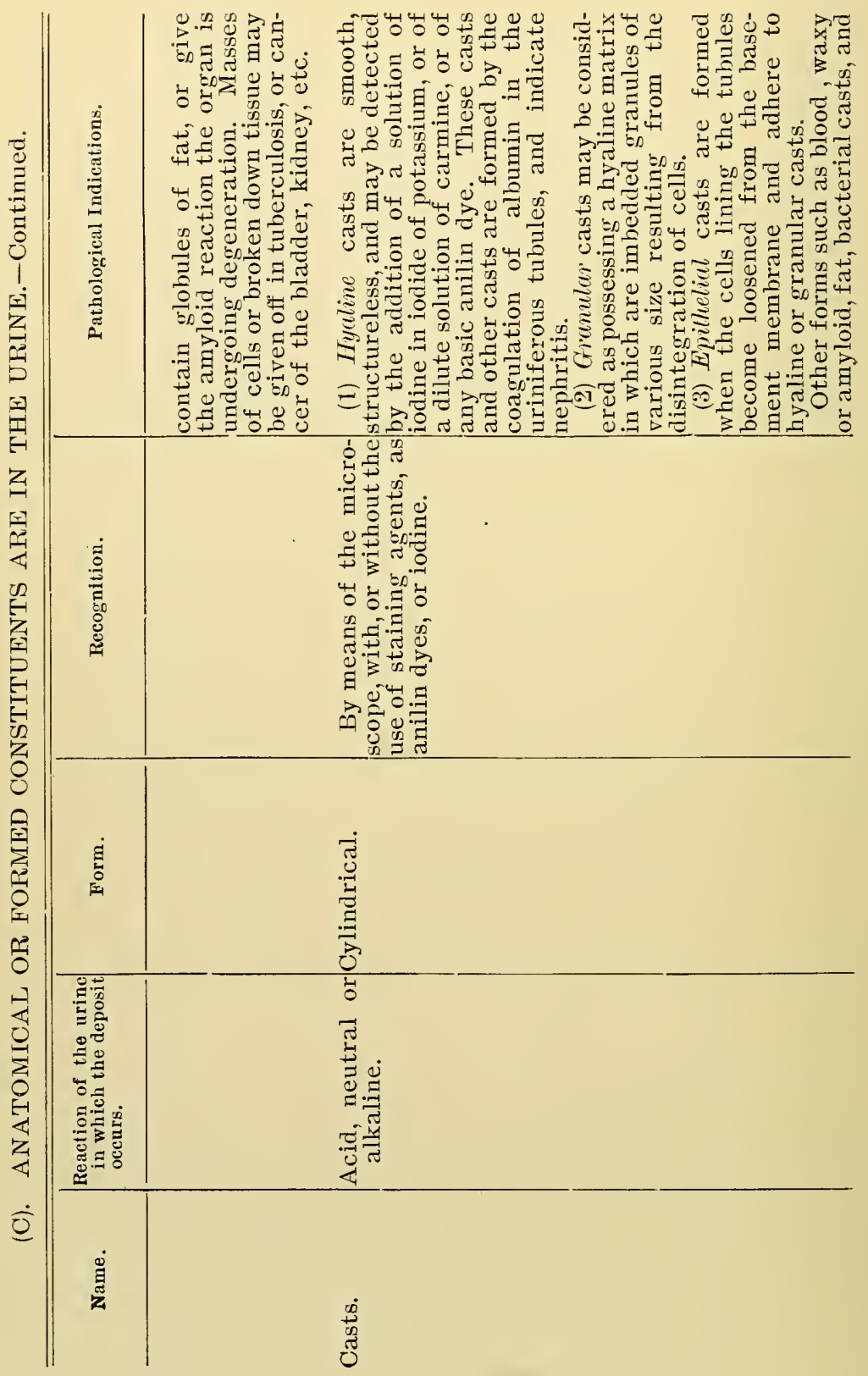




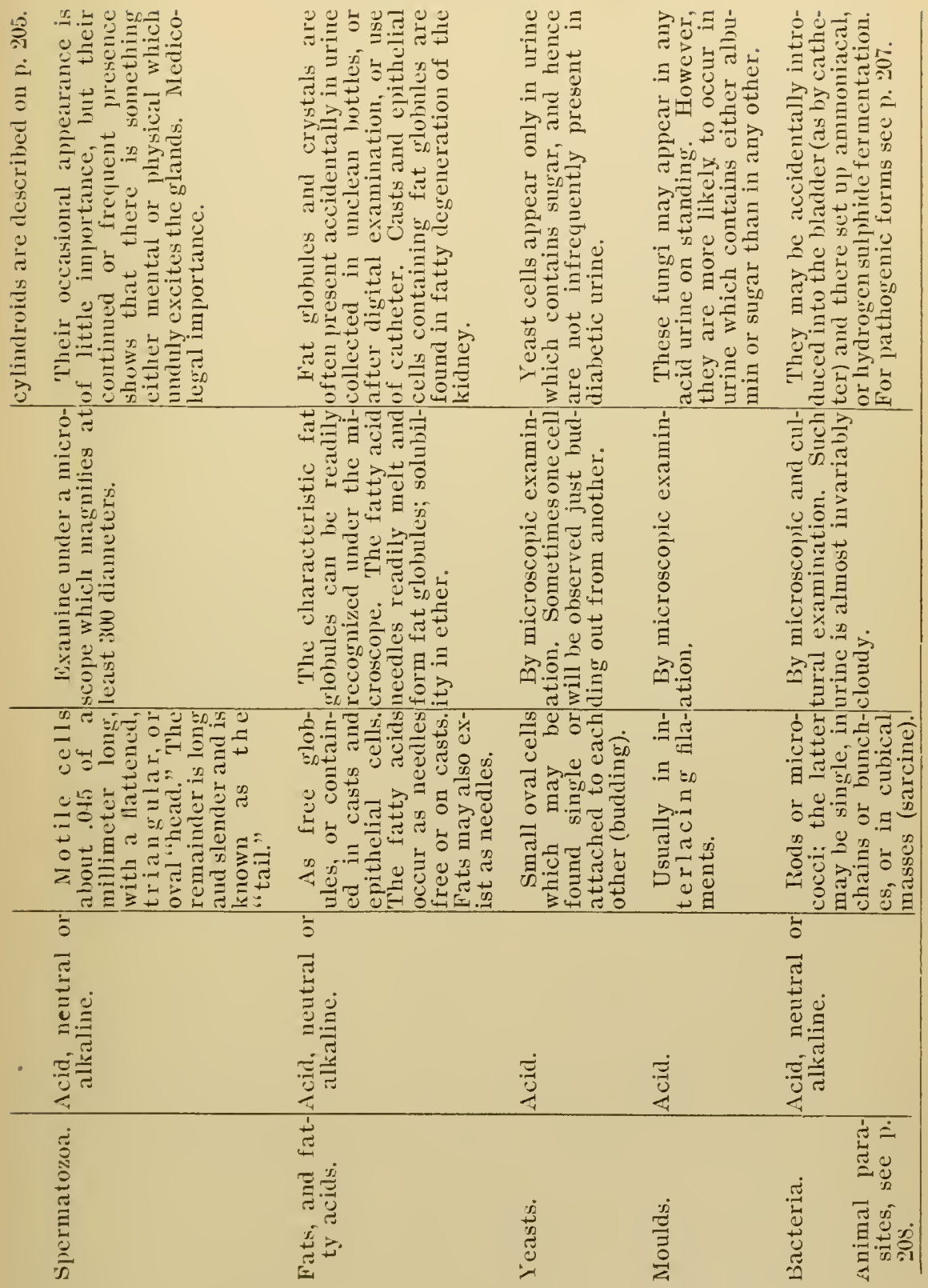




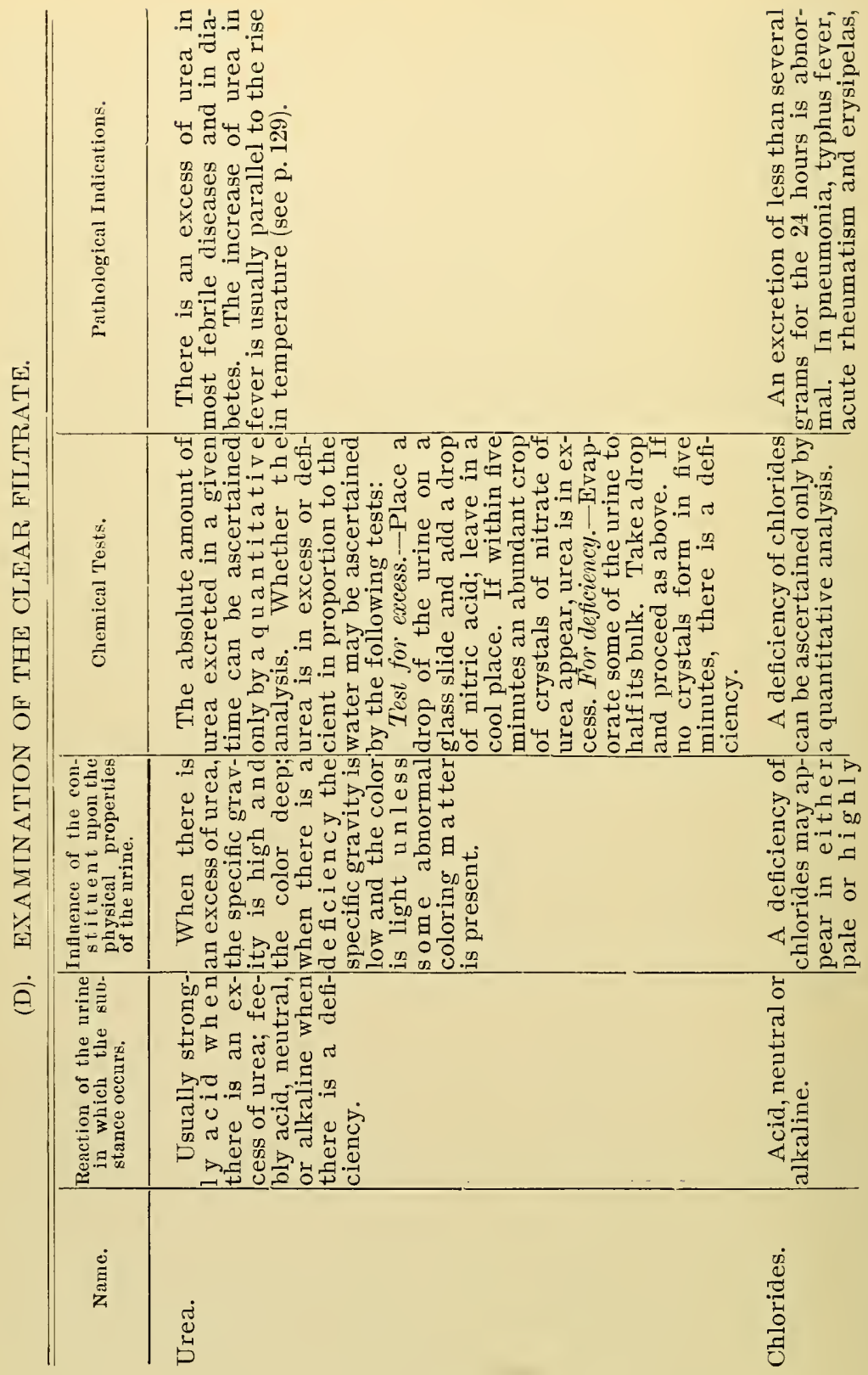




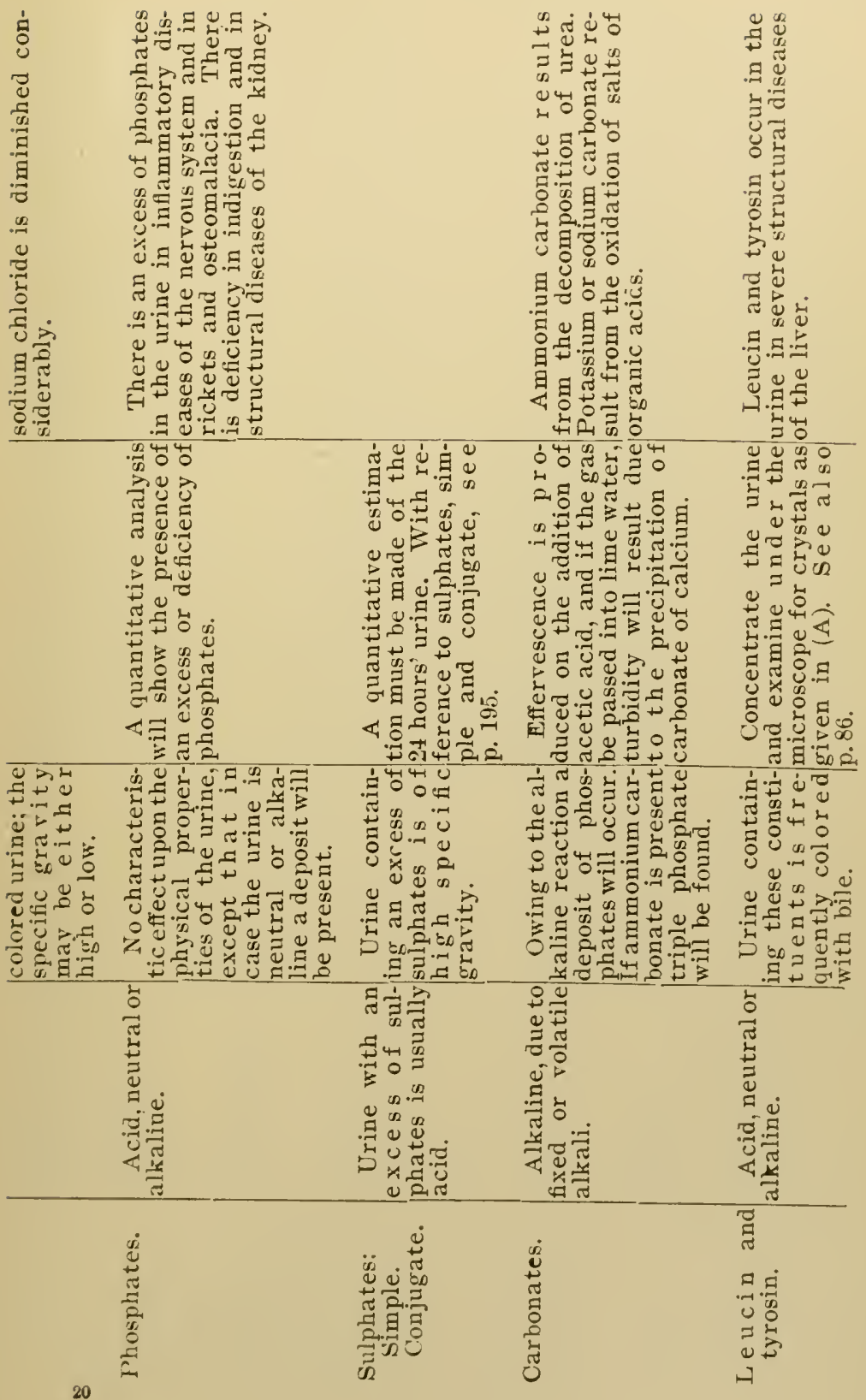


PHYSIOLOGICAC CHEMISTRY.

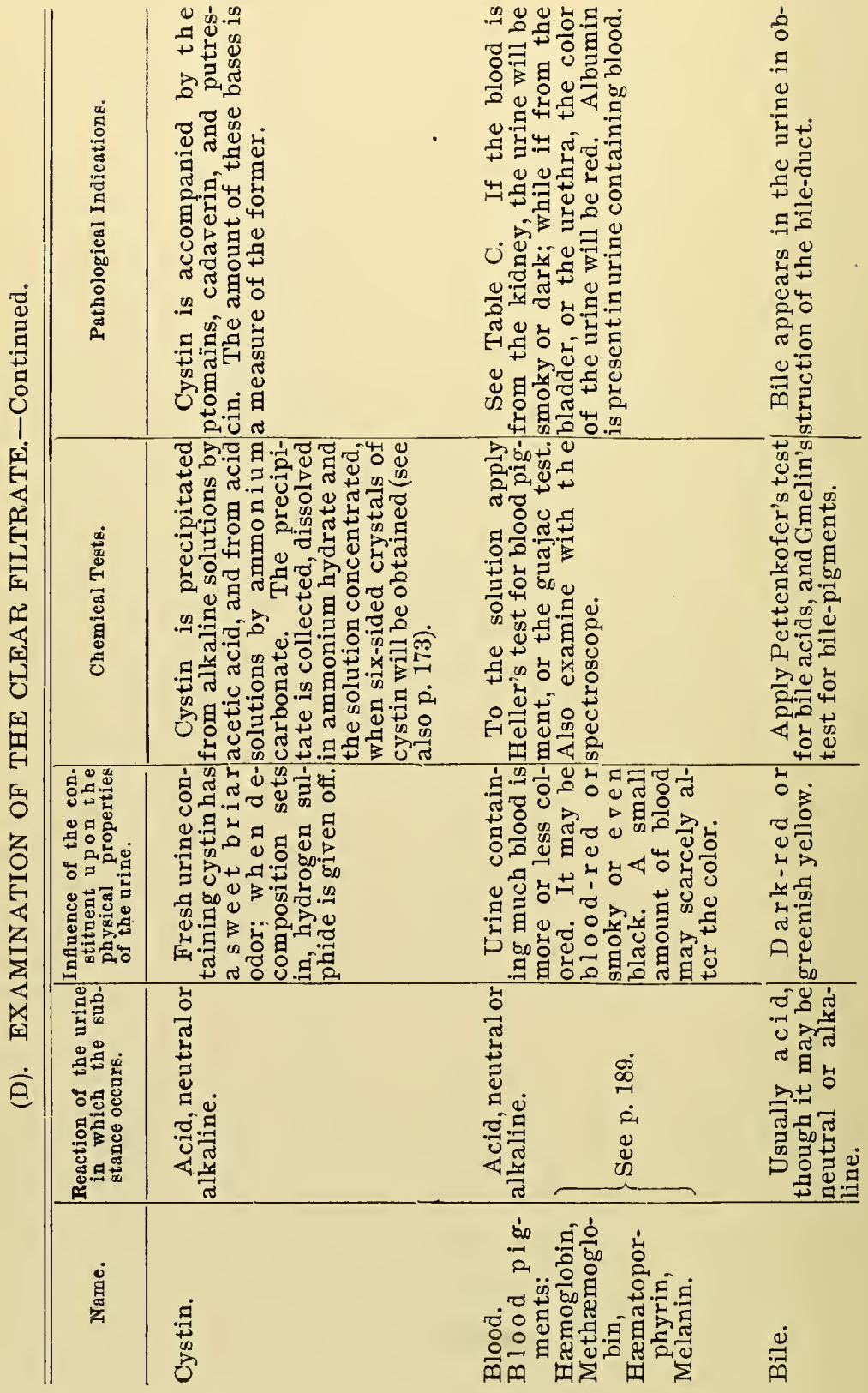




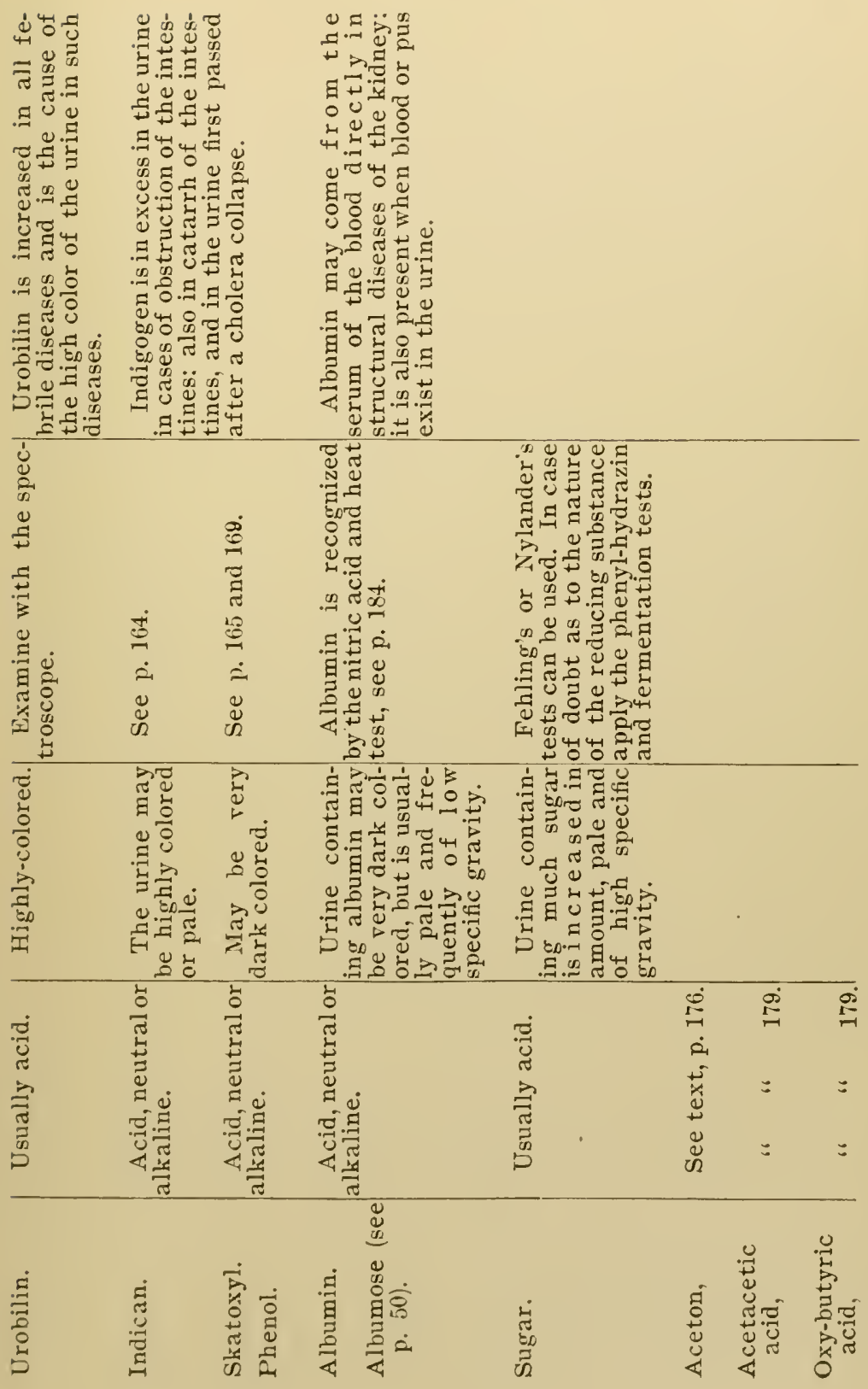


PHYSSIOLOGICAL CHEMISTRY.

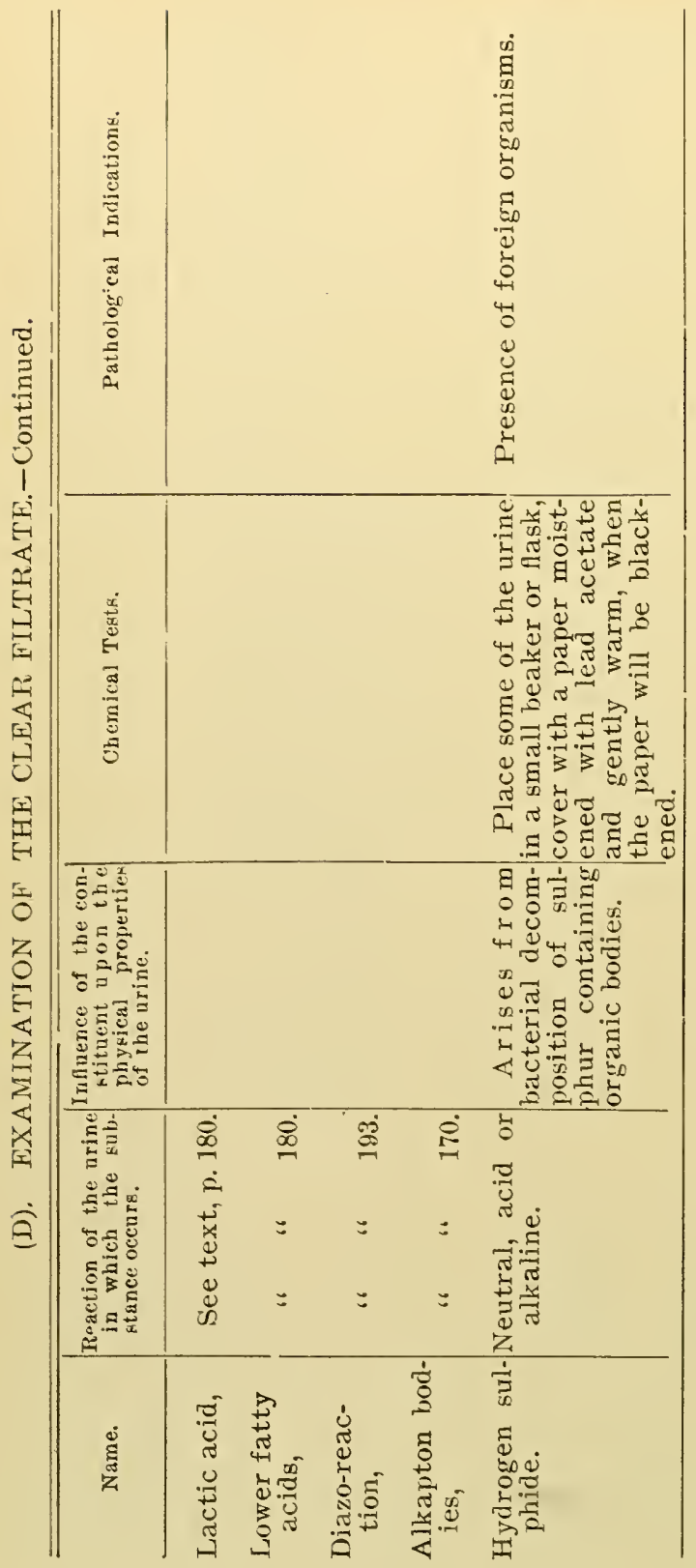




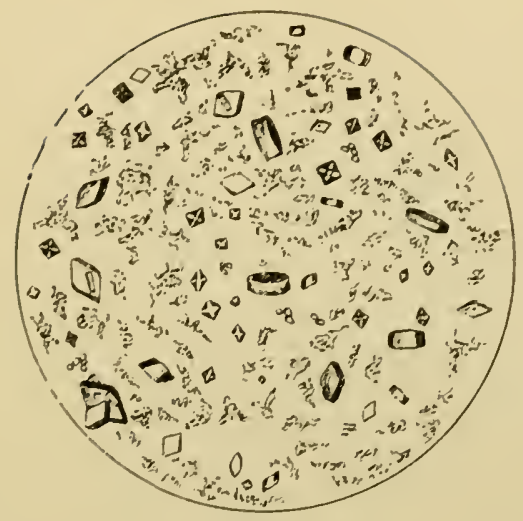

Fig. 11

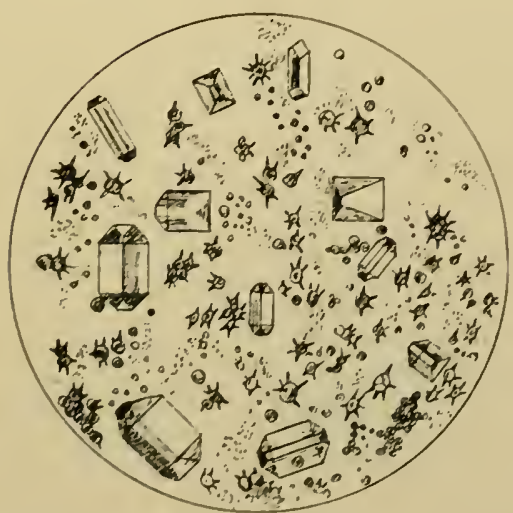

Fif; 10. Sediment in acid nrine.-Calcium oxalate, uric acid, and acorophous acid nrates. (NEUEAUZR and VOCEL.)

Fug!11. Sediment in alkuline nifine.-Triple and amorphons phosphates. ammoniurn urate and bacteria. (NEUBAUE⿺ and Vis $\in L$.) 

Fig. 12.

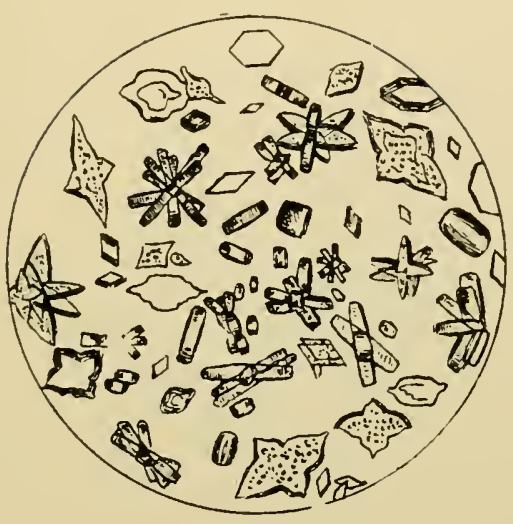

Fig. 13.

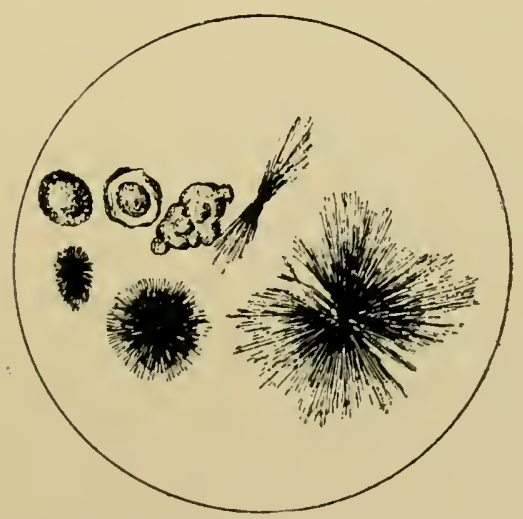

Fig. 12. Forms of uric acid. (Schotten.)

Fiw. 13. Lencin balls on the left-tyrosin needles on the right. (NecBauerand VOGEL.) 

Fig. 14 .

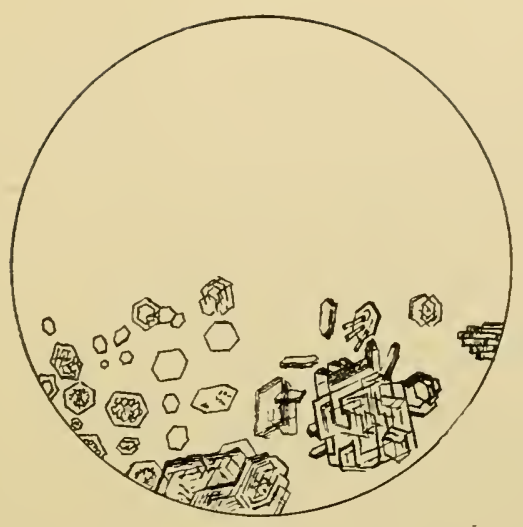

Fig. 15.

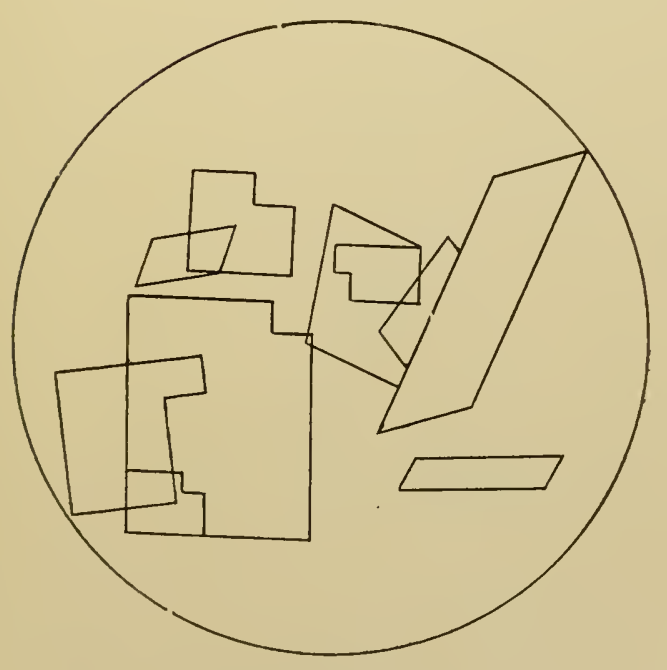

Fig. 14. Cystin. (Neubauer and Vogel.)

Fig. 15. Cholesterín. (Harley.) 

Fig. 16

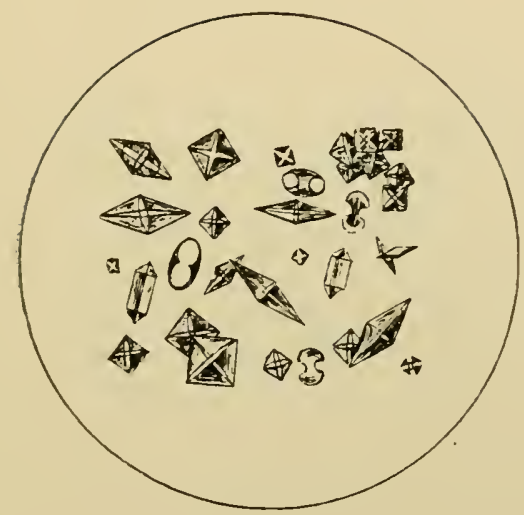

Fig. 17.

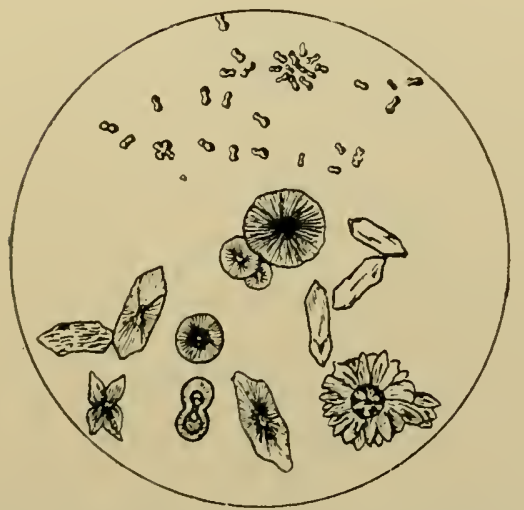

FIG. 16. Forms of calcium oxalate. (

Fig. 17. Forms of calcium carbonate. (VAUghaN.) 

Fig. 18.

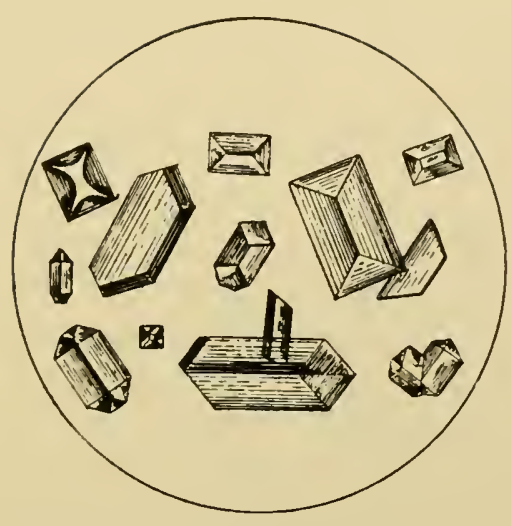

Fig. 19.

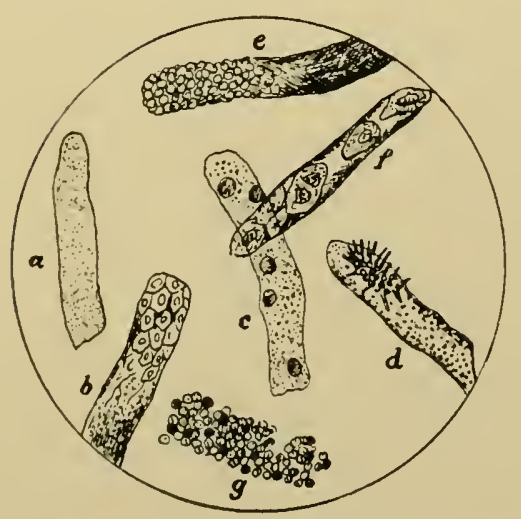

FIG. 18. Ammonjum magnesium phosphate (Triple phosphate), (

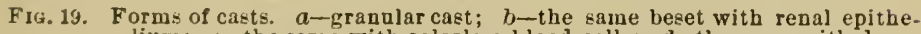
lium; c-the eame with colorless blood cells; d-the same with drops of fat and fatty crystals; e-hyaline cast covered with red blood cells; $f$-the thame with renal eithelium; $g$-cast of white blood cells. ( $\nabla$. JА KЕСH.) 

Fig. 20.

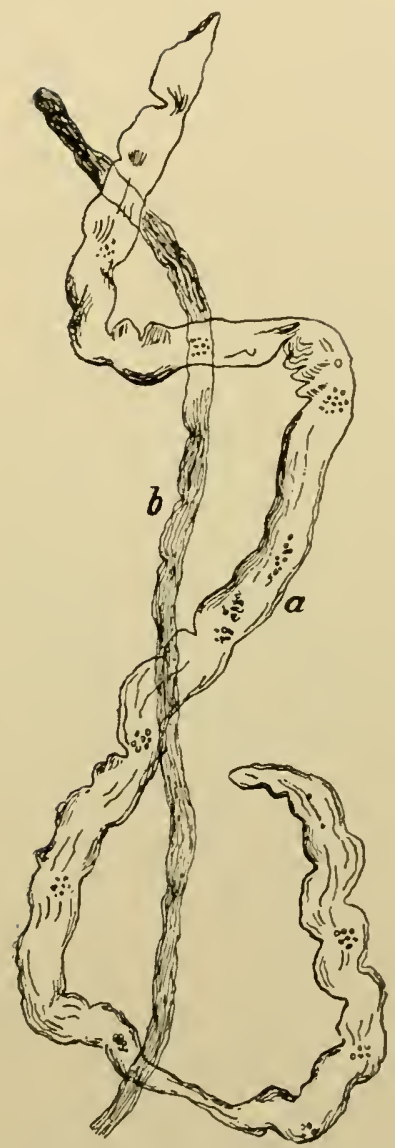

FIG. 20. Cylindroids from the urine in congested kidney. ( 

Fig. 21 .

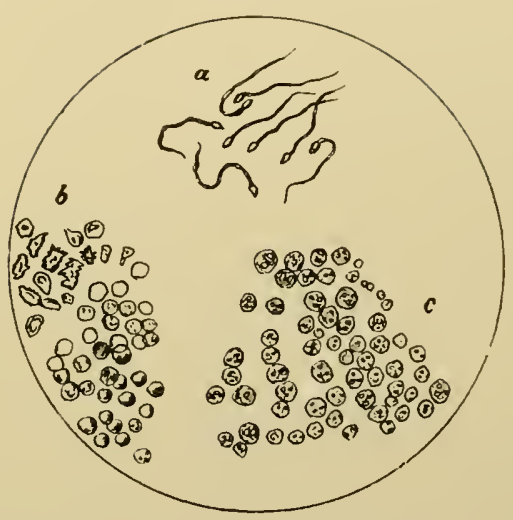

FIr. 21. $a$-spermatozoa: $b$-red blood cells, some crenated; $c$-pus corpuscles. (SchotTen, NeubaUEr and Vogel.) 

Fig. 22 .

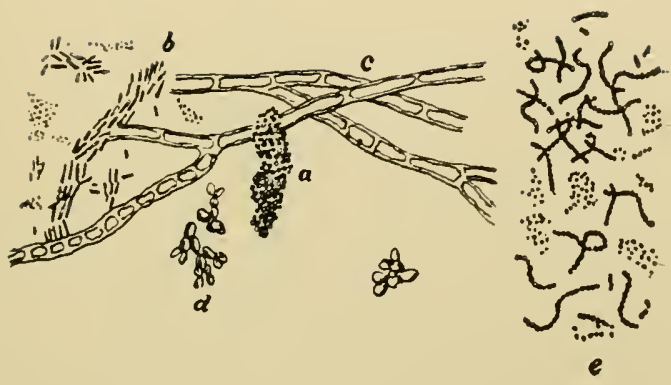

Fig. 23.

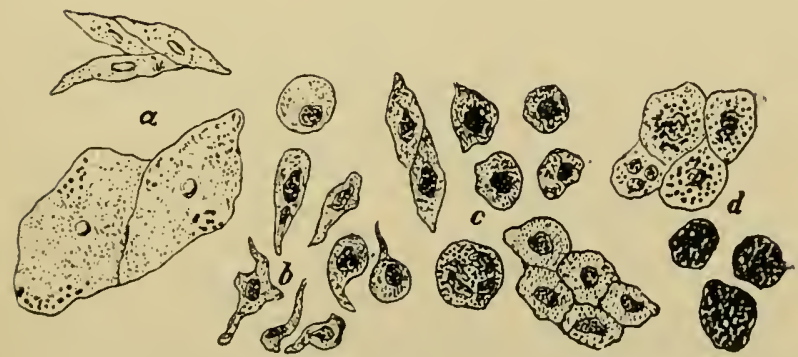

FIG. 22. $a$-micrococci; $b$-bacilli; $c$-filaments of moulds; $d$-yeast-cells; : e-micrococcus ureæ. (V. JА Бsсн.)

Fig. 23. $a$-Equamous eqjthelinm; $b$-epithelinm from the bladder; $c$-epithe- linm from the kidneys; $d$-fatty epithelium from the kidneys. ( $v$. $\mathrm{J}_{\triangle \mathrm{KSCH} \text {.) }}$ 



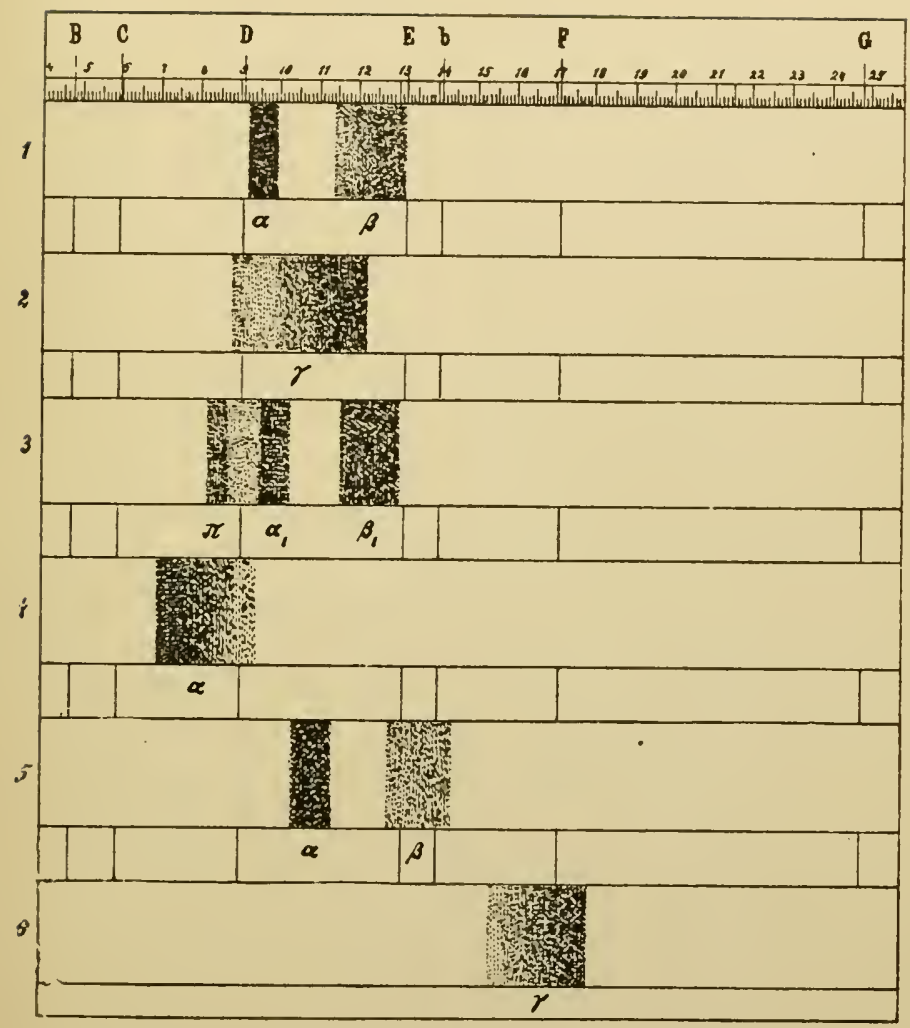

Fig. 24. Absorption spectra (after Hammansten):-1. A solution of oxy-hrmo. globin. 2. A solution of hremoglobin, obtained by treating oxy-hæmoglobin with an ammoniacal ferro-tartrate solntion (Stokes fuid). 3 A weak alkaline solution of methæmoglobin. 4. An alkaline solution of hrmatin. 5. An alkaline solution of hæmochromogen. obtained by treating an alkaline hematin solution with Stokes' fluid. 6 An acid solution of probilin. 



\section{List of Reagents.*}

Acid, Acetic, $\mathrm{HC}_{2} \mathrm{H}_{3} \mathrm{O}_{2}$. Glacial.

Acetic, dilute. Sp. gr. 1.04; about 30 per cent. acid.

Hydrochloric, HCl, concentrated. Sp. gr. 1.19-1.20; about 38-39 per cent. acid.

Hydrochloric, dilute. Sp. gr. 1.10; about 20 per cent. acid.

Hydrosulphuric, $\mathrm{H}_{2} \mathrm{~S}$. Used either in the form of gas, or saturated aqueous solution.

Iodic, $\mathrm{HIO}_{3} ; 1: 20$.

Nitric, fuming. Sp. gr. about 1.50.

Nitric, $\mathrm{HNO}_{3}$, concentrated. Sp. gr. 1.42; about $69-70$ per cent. acid.

Nitric, dilute. Sp. gr. 1.20; about 32-35 per cent. acid.

Oxalic, $\mathrm{H}_{2} \mathrm{C}_{2} \mathrm{O}_{4} \cdot 2 \mathrm{H}_{2} \mathrm{O} ; 1: 10$.

Oxalic, standard solution; see p. 239.

Phosphomolybdic; 1: 20.

Phosphotungstic; 1: 20.

Picric, $\mathrm{C}_{6} \mathrm{H}_{2}\left(\mathrm{NO}_{3}\right)_{3} \mathrm{OH}$; saturated solution; about $1: 100$. Rosolic, alcoholic solution; 1:100.

Sulphanilic; see p. 193.

Sulphuric, $\mathrm{H}_{2} \mathrm{SO}_{4}$, concentrated. Sp. gr. 1.84 .

Tannic, 1:100.

'Tannic, Almén; see p. 270.

Alcohol, $\mathrm{C}_{2} \mathrm{H}_{5} \mathrm{OH}$, absolute. Sp. gr. .79.

Alcohol, ordinary. Sp. gr. about .815; about 95 per cent. Ammoniacal Silver Nitrate; see p. 19 and 254 .

Ammonium Carbonate, $\left(\mathrm{NH}_{4}\right)_{2} \mathrm{CO}_{3} ; 1: 4$; add 1 part of $\mathrm{NH}_{4} \mathrm{OH}$, sp. gr. .96.

Ammonium Chloride, $\mathrm{NH}_{4} \mathrm{Cl} ; 1: 8$.

Ammonium Hydroxide, $\mathrm{NH}_{4} \mathrm{OH}$. Sp. gr. .96; about 10 per cent. $\mathrm{NH}_{3}$. 
Ammonium Molybdate, $\left(\mathrm{NH}_{4}\right)_{2} \mathrm{MoO}_{4}$. Dissolve 15 parts in 100 parts of hot water, and pour into 100 parts of $\mathrm{HNO}_{3}$ sp. gr. 1.20 .

Ammonium Oxalate, $\left(\mathrm{NH}_{4}\right)_{2} \mathrm{C}_{2} \mathrm{O}_{4} \cdot \mathrm{H}_{2} \mathrm{O} ; 1: 24$.

Ammonium Sulphate, $\left(\mathrm{NH}_{4}\right)_{2} \mathrm{SO}_{4}$; saturated solution, about $80: 100$.

Ammonium Sulphide, $\left(\mathrm{NH}_{4}\right)_{2} \mathrm{~S}$; saturate $\mathrm{NH}_{4} \mathrm{OH}$, sp. gr. .96 with hydrogen sulphide, then add an equal volume of $\mathrm{NH}_{4} \mathrm{OH}$, sp. gr. .96.

Barfoed's Reagent; see p. 20.

Barium Chloride, $\mathrm{BaCl}_{2} \cdot 2 \mathrm{H}_{2} \mathrm{O} ; 1: 10$.

Barium Chloride, standard solution; see p. 244.

Barium Hydrate, $\mathrm{Ba}(\mathrm{OH})_{2}$; saturated solution, 1:30.

Barium Nitrate, $\mathrm{Ba}\left(\mathrm{NO}_{3}\right)_{2} ; 1: 15$.

Baryta Mixture; see p. 251.

Boas' Reagent; see p. 69.

Bromine Water; saturated solution.

Calcium Chloride, $\mathrm{CaCl}_{2} ; 1: 8$.

Calcium Hydrate, $\mathrm{Ca}(\mathrm{OH})_{2}$; saturated solution.

Calcium Hypochlorite, $\mathrm{CaCl}_{2} \cdot \mathrm{Ca}(\mathrm{OCl})_{2}$; saturated solution, 1: 700 .

Chlorine Water; saturated solution.

Cleaning Mixture. Dissolve 80 parts of $\mathrm{K}_{2} \mathrm{Cr}_{2} \mathrm{O}_{7}$ in 350 c.c. of water, and then add cautiously 450 c.c. of $\mathrm{H}_{2} \mathrm{SO}_{4}$.

Cochineal. Dissolve 1 part in 100 parts of 25 per cent. alcohol.

Copper Sulphate, $\mathrm{CuSO}_{4}$. $5 \mathrm{H}_{2} \mathrm{O} ; 1$ : 8; one-half per cent. solution, or less, for biuret test.

Dimethyl amido-azobenzol; .5 per cent. alcoholic solution.

Ehrlich's Reagent; see p. 193.

Ether, $\left(\mathrm{C}_{2} \mathrm{H}_{5}\right)_{2} \mathrm{O}$. Sp. gr. about .725.

Fehling's Solution; see p. 247. 
Ferric Alum, $\mathrm{K}_{2} \mathrm{SO}_{4} \cdot \mathrm{Fe}_{2}\left(\mathrm{SO}_{4}\right)_{3}+24 \mathrm{H}_{2} \mathrm{O} ; 1: \Omega 0$.

Ferric Chloride, $\mathrm{FeCl}_{3}$; $1: 15$; almost colorless solution for lactic acid test.

Furfurol, concentrated; 1: 50.

Furfurol, dilute; 1:1000.

Guaiacum; 1:30; alcoholic solution. To be used fresh. Giinzburg's Reagent; see p. 68.

Iodine Solution (Lugol's solution); 1 part of iodine, 2 parts of potassium iodide, 300 parts of water.

Iodine Tincture; 7 per cent. alcoholic solution.

Lead Acetate, $\mathrm{Pb}\left(\mathrm{C}_{2} \mathrm{H}_{3} \mathrm{O}_{2}\right)_{2} .3 \mathrm{H}_{2} \mathrm{O} ; 1: 10$.

Lead Acetate, basic. Dissolve 17 parts of $\mathrm{Pb}\left(\mathrm{C}_{2} \mathrm{H}_{3} \mathrm{O}_{2}\right)_{2}$ in lot water, add 10 parts of lead oxide, boil for half an hour, dilute to 100 parts and filter.

Litmus Solution. Digest 1 part in 6 parts of water and filter.

Magnesia Mixture; see p. 254.

Magnesium Sulphate, $\mathrm{MgSO}_{4} \cdot 7 \mathrm{H}_{2} \mathrm{O}$; saturated solution, about $120: 100$.

Mercuric Chloride, $\mathrm{HgCl}_{2} ; 1: 16$.

Mercuric Nitrate, $\mathrm{Hg}\left(\mathrm{NO}_{3}\right)_{2}$; see p. 251.

Methyl Violet; .5 grams in 1000 . c.c. of water.

Millon's Reagent; see p. 40.

$\alpha$-Naphthol; alcololic solution, $15: 100$.

Naphthylamin Hydrochloride; saturated solution. To be used fresh.

Nylander's Solution; see p. 20.

Phenol Phthaleïn; alcoholic solution, 1:1000.

Potassium Alum, $\mathrm{K}_{2} \mathrm{SO}_{4} \cdot \mathrm{Al}_{2}\left(\mathrm{SO}_{4}\right)_{3}+24 \mathrm{H}_{2} \mathrm{O}$; saturated solution.

Potassium Chromate, $\mathrm{K}_{2} \mathrm{CrO}_{4} ; 1: 10$.

Potassium Dichromate, $\mathrm{K}_{2} \mathrm{Cr}_{2} \mathrm{O}_{7} ; 1: 10$. 
Potassium Ferricyanide, $\mathrm{K}_{3} \mathrm{Fe}(\mathrm{CN})_{6} ; 1: 12$.

Potassium Ferrocyanide, $\mathrm{K}_{4} \mathrm{Fe}(\mathrm{CN})_{6} .3 \mathrm{H}_{2} \mathrm{O} ; 1$ : 12 .

Potassium Hydroxide, KOH; $1: 10$.

Potassium Iodide, KI; 1 : 20.

Potassium Mercuric Iodide, (Mayer's Reagent); see p. 32.

Potassium Permanganate, $\mathrm{KMnO}_{4} ; 1: 20$.

Potassium Permanganate, standard solution; see p. 256.

Potassium Sulphide, $\mathrm{K}_{2} \mathrm{~S}$; see p. 254.

Potassium Sulphocyanate, KCNS; 1: 12.

Potassium Sulphocyanate, standard solution; see p. 241.

Schweizer's Reagent; see p. 35.

Silver Nitrate, $\mathrm{AgNO}_{3} ; 1: 20$.

Silver Nitrate, standard solution; see p. 241.

Sodium Acetate, $\mathrm{NaC}_{2} \mathrm{H}_{3} \mathrm{O}_{2} .3 \mathrm{H}_{2} \mathrm{O} ; 1: 5$.

Sodium Alizarin Sulphonic Acid; see p. 272.

Sodium Carbonate, $\mathrm{Na}_{2} \mathrm{CO}_{3} ; 1: 5$.

Sodium Carbonate, indicator; see p. 251.

Sodium Chloride, $\mathrm{NaCl}$; saturated solution, about 35: 100.

Sodium Chloride; $1: 10$.

Sodium Hydroxide, $\mathrm{NaOH} ; 1: 9$.

Sodium Hydroxide, for Kjeldahl distillation; 1 : 2.

Sodium Hydroxide, standard solution; see p. 239.

Sodium Hypobromite, NaBrO; see p. 252.

Sodium Indigo Sulphate, (Indigo Carmine); $1: 20$.

Sodium Nitrite, $\mathrm{NaNO}_{2} ; 1: 200$.

Sodium Nitroprusside; $1: 20$. To be used fresh.

Sodium Phosphate, $\mathrm{Na}_{2} \mathrm{HPO}_{4} .12 \mathrm{H}_{2} \mathrm{O} ; 1$ : 10 .

Sodium Sulphide, $\mathrm{Na}_{2} \mathrm{~S}$; see p. 254.

Starch Paste; see p. 28.

Stokes' Solution; see p. 102.

Tropæolin OO; see p. 69.

Uffelmann's Reagent; see p. 70.

Uranium Acetate; see p. 245.

Zinc Chloride, $\mathrm{ZnCl}_{2} ; 1$ : 20. 


\section{INDEX.}

Acetacetic acid....................179

Acetic acid.........................34, (i1

Aceton..................................176

Acetonuria ..........................176

Achromic point.. .................. 59

Achroodextrin...................15, 28

Acid albumin................38, 46, 65

" calcium phosphate........20(

" fermentation................2255

"، sulphur....................... 195

Acidity, estimation...............239

Acrolein.............................. 11

Acrylic aldehyde ................. 11

Adamkiewicz's reaction........... 40

Adenin .............................153

Adipocire .......................... 9

Albumin...................38, 42, 45, 183 " estimation...........261, 263

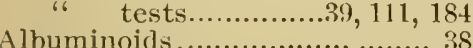

Albuminous borlies.................... 38

Albuminuria ...................... 184

Albumose...38, 42, 45, 49, 65, 79,

" detection........50, 186

"6 estimation..................264

، tests .......................... 50

Albumosuria ........................186,

Alimentary glycosuria..........17, 78

Alkali albuminate...............38, 46

Alkaline hæmatin..................104

" phosphates...............198

Alkalinity, estimation ............239

Alkaloidal reagents................. 44

Alkapton .. ......................16, 19

Alkaptonuria ....................... 170

Allantoic acid........................ 152

Allantoin................146, 150), 152

Allanturic acid..................... 152

Alloxan .............14.5, 146; 149, 152

Alloxanic acid................... 152

Alloxantin...............145, 148, 152

Alloxuric bases........................2ifi bodies......................256

Almén's guajac test...............1(1).

$\Lambda$ mirlo acids......................37, 126;

$\Lambda$ mido-barbituric acid............. 152

Ammonia................... $7,79,18(j$

Ammoniacal fermentation..... 137
Ammonium carbamate...........126 " carbonate............126 purpurate, see Murexid.

"6 urate calculi.......213

Ampho-pepton ...................... 66

Amylo-dextrin ......................... 15

Amyloid........................33, 35, 38 casts, see Waxy casts.

Amylopsin. 77

Amylum, see Starch.

Animal-gum.........................181

Animal-fat ........................... 7

Animal organisms.................208

Anti-albumose...................... 66

Anti-pepton......................66, 79

Anuria ................................226

Arginin ............................... 37

Asparaginic acid.................... 79

Bacteria............57, 206, 299, 313

Barbituric acid....................152

Barfoed's test.......................... 20

Benzoic acid..................156, 161

Benzopurpurin test ................69 69

Benzoyl chloride test..............133

Bile..................................... 137,193

"6 acids ...................... 88, 193

" " tests.......................... 90

" pigments ......................... 198

"

" stones ........................... 93

، " " analysis.................. 94

Bilicyanin........................... 89

Bilifuscin ................................. 89

Biliprasin .............................. 89

Bilirubin.........................88, 211

Biliverdin ........................... 88

Biuret................................134

"6 test .......................39, 134

Blood .............................97, 188

"6 casts...........................206

" coagulation...................100

" corpuscles .....97, 208, 277, 311

" examination.. ..................2273

" plasma ......................... 109

" plates............................... 99

" serum........................ 109 
Blood spectroscopic examination...................102, 315

Bloxam's test..........................133

Boas' test................................. 69

Böttger's test.......................... 20

Bromine reaction.................. 81

Butter ................................... 8 "6 fat............................. 12

Butyric acid................... $8,34,61$

Cadaverin......................172, 174

Caffein ..................................153

Calcium-bilirubin .................. 93

Calcium carbonate............210, 305 "6 "6 calculi........213 " oxalate..175, 210, 299, 305 " " calculi........ 213 " phosphate..........199, 200 "، sulphate.....................210

Cancer cell..........................204 Cane sugar...................15, 16, 22 Caramel ............................... 18

Carbamic acid......................127

Carbamide, see Urea.

Carbohydrates.. ................15, 181 6 $\quad$ absorption........ 78

Carbolic urine.........................168

Carbon monoxide hæmoglobin

104,106

Casein ...............................38, 116

Casts.......................184, 204, 307

Cellulose.............................15, 32

Cercomonas ...........................208

Chlorides...............................200

" estimation................ 241

Cholesterin. $\ldots 88,90,93,94,180,211,303$

Cholesterin calculi........93, 95, 214 " tests.................... 95

Cholic acid................................ 88

Chrysophanic acid .................... 20

Chyle ....................................... 10,99

Chyluria...............................180

Chymosin, see Rennin.

Coagulation point.................. 45

test .................. 43

Collagen ............................38, 79

Collodium ................................ 34

Colostrum corpuscles.................118

Congo-red test....................... 69

Colorimetric method... ...........274

Conjugate sulphates ................195 estimation..245

Coprosterin ........................... 95

Creatin...................................153

Creatinin.............................153

Cresol, see Phenol.
Cyanuric acid ......................134

Cylindroids....................206, 309

Cysteïn ..............................172

Cystin.......................172, 210, 303 calculi .................174, 214

Cystinuria. 172

Cystosin..................................192

Czapek's method.....................255

Defibrinated blood............100, 102

Densimetric method ..............261

Dentero-albumose...............53, 66

Devoto's method ................... 52

Dextran................................. 15

Dextrin .................................. 29

Dextrose................15, 16, 17, 182 " estimation................ 247 tests ..................... 17

Diacetic acid, see Acetacetic acid.

Diaceturia 179

Dialuramid .........................145, 152

Dialuric acid.....................145, 152

Dialysis ............................. 48

Diamines .... ......................... 174

Diastase.......................25, 38, 56

Diazo reaction ................... 193

Di-methyl amido-azobenzol test 68

Di-saccharides ....................... 15

Distomum hæmatobium...........208

Donné's test.........................191

Doremus' method .....................253

Drechsel's reaction...................145

Dumas' method... .................260

Dynamite ........................... 14

Dys-albumose....................... 65

Dys-pepton ......................... 64

Earthy phosphates..................198

Echinococci ......................... 208

Egg albumin.....................38, 39 "6 globulin ..................... 38

Ehrlich's reaction..................... 193

Einhorn's saccharimeter.........250

Elastin ................................ 38

Empirical solution..................218

Enzymes.............................. 38

Epithelial casts......................205 cells ...............202, 319

Erythrodextrin....................15, 28

Esbach's albuminometer ........262

Ethereal sulphates................195

Exudates................................. 99

Factor ...............................221

Fats...................... 7, 88, 118, 180

" absorption ........................, 77

" emulsification....8, $9,13,76,80$ 
Fats preparation................10, 79 .. saponification................ 1: Fat-splitting ferment, see Steapsin.

Fatty acid ......................12, 27 .. casts .........................206

Fehling's solution ....................2t7 test ....................... 19

Fermentation test .................. 21

Ferrocyanide test...............43, 185 Fibrin...................3s, 100, 113, 1ss ferment ...............100, 114

Fibrinogen.............38, 99, 113, 183

Filaria sanguinis..................208

Fixed alkali...........................236

Fleischl's haemometer...........274

Folin's method ........................25ti

Formic acid.......................... 24

Fructose, see Lavulose.

Furfurol test. ..................91, 133

Galactite

22

Galactose.......................17, 22, 24

Gastric juice........................ 61 " analysis........74, 272 Gelatin .............................38, 54 " pepton..................... 79

Gerhard t's test.......................179 Globulin..............38, 42, 45, 47, 183 "detecíion ................. ts ، estimation...........261, 263

Glucose, see Dextrose.

Glucosin.............................. 26

Glycerin........................ $7,13,76$ phosphoric acid.........198

Glycocholic acid.................... 88 Glycocoll....................88, 156, 160 G'lycogen .........................15, 29 Glyco-proteid ....................... 38

Glycose................................ 15

(Hlycosuria............................182

Glycuronic acid..............16, 157, 183

Glyoxyl-diureid, see Allantoin.

Glyoxyl urea, see Allanturic acid.

Gmelin's test 92

Gionococcus............................ 207

Granular casts...................... 205

Grape sugar, see Dextrose.

Guajac test...............105, 120, 191

Guanin................................... 153

Gun cotton.......................... 34

Gunning's test........................178

Gïnzburg's test..................... 6s

Hamatin................42, 89, 107

Hematoidin.......................... 89

Hærnatoporphyrin............104, 189
Hamaturia .. ......................188

Hxmin..............................107 crystal test............9S, 107 Hæmochromogen.............103, 107 Hamoglobin................38, 42, 189 estimation...........27t

Hamoglobinuria ...................189

Hammarsten's method............263 Haycraft's method.................255

Heintz's method.....................254

Heller's test......................42, 108

Hemi-albumose..................... $66^{\circ}$

Hemi-pepton.......................66, 79

Hetero-albumose.............65, 186

Heteroxanthin ......................153

Hexose.............................15, 16

Hippuric acid..................156, 209

". "tests................160

Histidin ............................... 37

Histon.................................38, 187

Histozym............................... 161

Hoffmann's reaction............... 85

Hofmeister's method.............. 51

Homogentisinic acid.........170, 183

Hopkin's method...................25

Hoppe-Seyler's test............91, 106

Hüfner's method....................25'2

Humin substances............. 17, 24

Huppert's reaction.................. 92

Hyaline casts.........................204

Hydantoic acid.....................152

Hydrobilirubin ..................... 89

Hydrochloric acid................61, 62

$$
\text { "6 } 3 \text { " }
$$
bined....................61, 62

Hydrogen sulphide..................196

Hydroquinon ........................171

Hydrothionuria ........................197

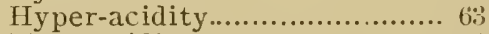

Hypo-acidity ....................... $6: 3$

Hyposulphurous acid................198

Hypoxanthin.........................15i

Indican, see Indoxyl.

Indigo......................19, 162, 211

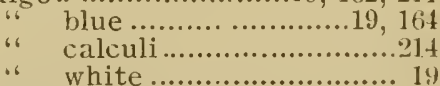

Indigogen, see Indoxyl.

Indol.............................162, 164

Indoxyl ............................... $16_{2}^{*}$

" $\quad$ red............................... 16:3

test .........................10t

Inulin.................................22, 26

Invert sugar . ........................ 
Invertin ......................23, 38, 78

Iodine test........................ 92

Iodoform test...................... 178

Iso-maltose.................15, 26, 28

Jaffe's test .........................164

Jolles' test ........................... 93

Kephir

Keratin ............................... 38

Kjeldahl method........................258

Kumyss... ........................... 25

Lact-albumin ...................38, 117

Lactic acid...................25, 61, 116

" " 6 test ................ 70

Lacto-caramel ....................... 24

Lacto-globulin........................38, 117

Lactose ....................16, 24, 118

Lactosozon.......................... 25

Lævulin................................ 26

Lavulinic acid ................17, 1s, 24

Lævulose.................... 15, 16, 22

Laky blood ....................98, 106

Lard ............................... 7,12

Lead plaster ......................... 13

Lecith-albumin......................235

Lecithin............................... s8

Legal's test .............................178

Leucin.........37, 66, 79, 82, 175, 301

"6 tests.........................83, 86

Leucocytes ..........................98, 203

Lieben's test .........................178

Liebermann's reaction............ 40 "6 cholesterol reaction 95

Liebig's method........................251

Lipaciduria .........................180

Lipuria.................................180

List of reagents.......................317

Lithemia .............................141

Ludwig's method.... ...............254

Lymph........................10, 99, 125

Lymphoid cells....................... 98

Lysidin..................................143

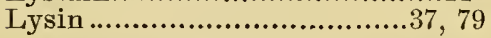

Magnesium ammonium phosphate............200, 299, 307

Malonyl urea, see Barbituric acid.

Maltodextrin...................... 28

Maltose..................15, 16, 25, 28

Maltosozon ............................ 26

Mannite...............................15, 16

Margaric acid...................... 10

Margarine............................. 10

Marsh gas................................. 34

Melting point, determination..131
Mesoxalic acid......................149

Mesoxalyl urea, see Alloxan.

Methæmoglobin..............108, 190

Methyl violet test..................... 69

Micrococcus ureæ..............207, 313

Milk ................................116

" analysis......................267

" composition ..............116, 271

" fat...........................8, 118

" serum .......................116

" sugar, see Lactose.

" tests...........................119

Millon's reaction..................... 39

Mitscherlich polarimeter........ 35

Mohr's method.........................242

Molisch's reaction................... 17

Mono-saccharides................15, 16

Moore-Heller test.................... 18

Mörner-Sjöqvist method ........ 253

Mörner's test.........................179

Moulds ..........................208, 313

Mucic acid........................22, 25

Mucin...........38, 57, 58, 87, 183, 187

Mucoid ............................... 38

Mucous corpuscles ....................203

Murexid..........................145, 152

"6 test.............................. 145

Muscle albumin ........................ 38

Myosin .............................. 38

Neubauer's method .........155, 233

Neutral calcium phosphate.....199 "6 sulphur...............195, 197

Nitric acid test...........43, 111, 185

Nitro-cellulose ...................... 34

Nitro-glycerin.......................... 14

Nitroprusside test.................178

Nitrogen, estimation...............258

Nitrous acid........................... 58

Normal solution.........................220

Nuclein... . . .....................38, 64 "6 bases, see Xanthin

bases.

Nucleinic acid,.....................192

Nucleo-albumin.........38, 42, 87, 187

Nucleo-histon....................38, 191

Nucleo-proteid ..................... 64

Nylander's test...................... 20

Oleic acid.............................. 12

Olein...................................... 7

Oleomargarine....................... 10

Oliguria.....................................2226

Organized sediment....................202

Ornithin ................................158

Ornithuric acid ........................158

Osazon ....................................... 21

Oxalate plasma......................100 
Oxalic acid ................ $28,146,175$ ()xaluria ............................ Oxaluric acid................ 14t, 15: Oxalyl urea, see l'arabanic acid.

Oxidized sulphur...................195

Oxy-butyric acid..................179

Oxy-haemoglobin ..................102

Oxyuris vermicularis............208

Palmitic acid ...................... 10

Palmitin .............................. 7

Pancreatic secretion............ 76

Papayotin .......................3s, it

Parabanic acid...............146, 15:2

Para-casein.................s, 117, 120

I'ara-glohulin, see Serum globulin.

Para-nuclein ........................ 17

Paraxanthin.........................531

Parchment .......................3:3, 35

Pavy's solution....................... 20

Pentite............................. 16

Pentosane .......................15, 16;

Pentose. ..................15), 16, 181

Pentosuria............................1s1

Penzoldt's test......................178

Pepsin.....................88, (1), 121 test....................... 74

Pepsin-hydrochloric acid........ 62

Peptic digestion................. 71

Pepton...38, 42, 45, 51, 66, 79, 99, 186 detection ................ 51

Pettenkofer's test................... 90

Phenol....................162, 16it, 169

Phenyl-glucosozon................ 21

Phenyl-hydrazine test ........... 21

Phloro-glucin vanillin test..... 68

1'hosphates....................210, 299

Phosphatic calculi.................213

Pliosphoric acid....................198

66 estimation....245

Phytosterin.......................... 95

Phospho-sarkinic acid........... 67

Pialyn, see Steapsin.

Piperazin 143

piria's reaction..................... 85

Plant albumin..................... \&s globulin....................... 38

T'ohl's method.......................26;3

l'oly-salccharides................ 15

l'olyuria ........................184, 220

Potassium phenol sulphate..... I is

l'rimary albumose............... 65 66 (6)

l'roteids.............................. ;8

"6 tests..................., :9

${ }^{\prime} r o t e i n s \ldots \ldots \ldots \ldots \ldots \ldots \ldots \ldots \ldots \ldots \ldots \ldots, 37$
Proteins classification........... is

Proteinochrom..................... 81

Proteinochromogen..........7!

Proteose . ...................... 66

Prothrombin................ 101, 114

Proto-albumose ................... (65

Pseudo-casts ..................... 20t;

Pseudo-nuclein..............6t, 117

Ptyalin ...........................56, 59

Ptyalism ........................ 5i)

Purpuric acid..................145, 15:

Pus ...................105, 190, 203, :311

"6 casts......................... 206

Putrescin ...................173, 174

Pyrocatechin .................25, 169

Pyroxylin ............................ 3t

Pyuria............................190

Quantitative analysis............217

$$
\begin{aligned}
& \text { "6 of blood..273 } \\
& \because 6 \quad \text { analysis of milk ... } 67
\end{aligned}
$$

Iieduced hæmatin...................10:;

"6 hamoglobin................102

Rennin ........................8s, 61,67

lihamnose .......................... 15

Roberts-Stolnikofl method......262

liosenbach's test.................. 92

Saccharic acid ..............25, 28

Saccharide......................... 15

Saccharose, see Cane sugar.

Saliva................ .............. 55

Salivary calculi..................... 56

Sarkinic acid......................... 67

Scherer's method......................261

test......................84, 85

Schweizer's reagent............... 34

Secondary albumose............... 66 "6 calculi....................212

Serum albumin...............88, 47,99 " grlobulin..................8, $47,99,110$ Skatol … ......................162, $16 \pi$

Skatoxyl ..............................

skeltal substances.................. 38

Soap.........................13, 76,88 Soleil-Ventzke saccharimeter. 83 Soluble starch.................27, 28 Specific gravity, determination

Spermatozoa..................204, 211

standard solutions..................218

Stitrch............................15, 26

“ granules..................... 27

"، iodide......................... 28 
Starch paste ...................... 28 sugar, see Dextrose.

Steapsin ..................9, 38, 76, 80

Stearic acid......................... 10

Stearin................................ 7

Stercobilin.......................... 89

Stercorin............................ 95

Stokes' reagent......................102

Stomach contents, examination

Stroma.............................. 98

Sucrose, see Cane sugar.

Suet

Sulphocyar

Sulphocyanate...................58, 194

Sulphur .............................194

Sulphur-methæmoglobin ..........106

Sulphuric acid .......................197

" " estimation ......244

Syntonin, see Acid albumin.

Table of atomic weights........216

'Tallow................................. .7, 12

Tartar ...................................... 56

Tartaric acid ......................... 28

Tartronyl urea, see Dialuric acid.

Taurin ...........................88, 195

Taurocholic acid...................... 88

Thein ...................................... 153

Theobromin..................................153

Thiosulphuric acid...................198

Thrombosin.............................101

Thymic acid...............................192

Thymin .................................192

Tissue débris..........................204

Toepfer's method ....................272

Transudates............................ 99

'Trichomonas vaginalis............208

'Triple phosphate, see Magnesium ammonium phosphate.

Trommer's test...................... 19

Tropzolin OO test.................... 69

Trypsin..............................38, 78

Trypsinogen........................ 78

Tryptophan ............................. 81

Tubercle bacillus ................... 207

Tunicin................................. 32

Tyrosin...37, 66, 79, 84, 175, 210, 30 L " tests....................85, 86

Uffelmann's test.................... 70

Unknowns....................75, 277, 280

Unorganized sediment .............209

Unoxidized sulphur....................195

Uræmia .................................128

Uramil, see Dialuramid.

Urase
Urates ........................209, 299

Urea.. ..........................8s, 125

" detection ........................136

" estimation........................251

" nitrate................................132

"، oxalate ...............................132

" preparation.......................129

"، properties ........................131

"6 tests.................................133

Uric acid............................

$125,138,152,153,209,299,301$

، " " calculi......................213

‘, ، "

$\therefore$ " " 4 preparation ................. 142

" " " " 6 properties.................143

"، " tests............................144

Urinary calculi ....................211

"6 "6 analysis...........214

، sediment ............201, 283

Urine.....................................123

" composition...............265, 266

" examination .....................283

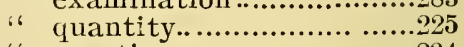

"6 reaction ...........................234

“ specific gravity............230, 253

" total solids....................233

Urobilin.................................. 89

Urocyanin...............................165

Uroglaucin.............................. 165

Uroleucinic acid...................170, 183

Urohæmatin .............................165

Urophæin.............................124

Uroroseïn ..............................165

Urorubin ...............................165

Urostealith ..........................214

Varrentrapp-Will method.......260

Vegetable oils...................... 7

organisms..............206

Vitali's guajac test..................191

Vitellin ................................. 38

Volatile alkali..................138, 236

Volhard's method ....................241

Waxy casts ..........................205

Whey ....................................116

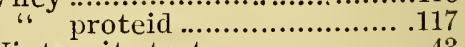

Winternitz test ...................... 43

Wood-silk .............................. 34

Xanthin................139, 153, 211

" bases....................139, 153

"، calculi.......................214

Xantho-creatinin .......................155

Xantho-proteic reaction......... 40

Yeast cells......................208, 303 




\section{A LIST OF BOOKS}

PUBLISHED BY

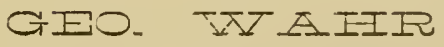

Publisher and Bookseler to the UNiversity of MrChigan, ANN IRBOR.

Any book in this list will be sent, carriage free, to any address in the world on receipt of price named.

BOWEN.-A Teachers' Course in Physical Training. By Wilbur $P$. Bowen, Director of Physical Training, Michigan State Normal College. In Press.

CHEEVER.-Select Methods in Inorganic Quntitatize Analysis. By Byron W. Cheever, A.M., M.D., late Acting Professor of Metallurgy in the University of Michigan. Revised and enlarged by Frank Clemes Smith, Professor of Geology, Mining and Metallurgy in the State School of Mines, Rapid City, S. D. Parts I. and II. Third edition. I2mo. \$1.75.

The first part of this book, as indicated by the title, consists of Laboratory Notes for a Beginner's Course in Quantitative Analysis. It considers the subjects of Gravimetric and Volumetric Analysis, for beginners, by means of the chemical analysis of a set of substances. properly numbered, in each case giving the methods to be followed in such analysis; also the methods for calculating and preparing volutnetric standard solutions, generally following the course offered by Professor Cheever to his students. It also considers the methods for the determination of the specific gravities of various liquids and solids.

Although a number of the analyses contained in Part I. may be of only approximate accuracy, and of small commercial value, such are yet included with a special purpose, to wit:- that they may supply the student with a wider range of work and a greater diversity of chemical manipulation. This was Professor Cheever's idea, and it is certainly a good one, especially since, in most cases, the work of the beginner simply serves to emphasize the necessity of careful scrutiny of details and methods for practical work in the future.

Part $\mathrm{I}$, is offered, then, for the use of schools and colleges, and it is intended to supply a source of elenentary information upon the subject of Quantitative Chemical Analysis rarely oflered in such form in works upon that subject.-Preface.

The author was for inany years Professor of Mletallurgy in the Uoiversity of Michigan, and the methods here presented are those mostiy offered by him to his students. As a beginner's book in quantitative analysis, it will be found eminently practical, and it can be honestly recominende-l to the student who desires a source of elementary information upon this branch of applied science The book is divided into two paris, the first consisting of laboratory notes for beginners. Tlie subjects of gravimetric and volumetric analysis are considered by means of the chemical analysis of a set of substances, propecly numbered, in eacli case giving the methods to be followed in such analysis, and also the methods ol calculating and preparing volumetric standard solutions, etc. Metlods for the determination of specific kravities of various liquids and solids are also considered.

Part 11. contains a uumber of select methods in inorganic quantitative analysis. such as the analysis of limestone, iron ores, manganese ores, stecl, the analysis of 
coal, water, mineral plısplıates, smeling ores, lear slags, copper, arsenic, bismut $\mathbf{b}$, etc. A chapter on reagents concludes the work.-Pharriaceucicil Ela.

DEWEY.-The Study of Ethics. A Syllabus. By Jonn Dewey, Professor of Philosophy in the University of Chicago. Octavo. I44 pages. Cloth, \$I.25.

D'OOGE.-Helps to the Study of Classical Mythology; for the Lower Grades and Secondary Schools. By B. L. D'Ooge, Professor in the Michigan State Normal College. I 2 mo. ISo pages. Cloth. 45 cents.

A bibliography based on practical experience. The author is a professor in the Michigan State Normal College. As the myths of all narions manifest themselves first in religion, secondly in art, and third in literature, these reading references are grouped in the above classes. One section is devoted to the study of mythology in the grades, and an introductory chapter gives hints for teaching the subject in the lower grades. The books suggested in the body of the work are given in one alphabet at the end, with publishers and prices; there are also blank pages for additional references, and a good general index.-Publishers Weekly,

DOW.-Brief Outlines in European History. A Syllabus for the Use of Students in History, Course I., in the University of Michigan. By Earl Wilbur Dow. 4 I pages. Pamphlet, 35 cents.

DOW.-Brief Outlines in European Fistory. A Syllabus for the Use of Students in History, Course II., in the University of Michigan. By Earl Wilbur Dow. 47 pages. Pamphlet, 35 cents.

DZIOBEK. - Mathematical Theories of Planetary Motions. By Dr. Otto Dziobek, Privatdocent in the Royal Technical High School of Berlin, Charlottenburg. Translated by Mark W. Harrington, formerly Chief of the United States Weather Bureau, and Professor of Astronomy and Director of the Observatory at the the University of Michigan, President of the University of Washington, and Wm. J. Hnssey, Assistant Professor of Astronomy in the Leland Stanford, Jr. University. 8vo. 294 pages. $\$ 3.50$.

The determination of the motions of the heavenly bodies is an important problem in and for itself, and also on account of the influence it has exeried on the development of mathematics. It has engaged the attention of the greatest mathematicians, and, in the course of their not alrogether successful attempts to solve it, they have displayed unsurpassed ingenuity. The methods devised by them have proved useful, not only in this problem, but have also largely determined the course of advance in other branches of mathematics. Analytical mechanics, beginning with Newton, and receiving a finished clearness from Lagrange, is especially indebted to this problem, and in turn, analytical mechanics has been so suggestive in method as to determine largely both the direction and rapidity of the advancement of mathematical scienoe.

Hence, when it is desired to illustrate the abstract tbeories of analytical mechanics, the profundity of the mathematics of the problem of the motions of the heavenly bodies, its powerful influence on the historical development of this science, and finally the dignity of its object, all point to it as most suilable for this purpose.

This work is intended not merely as an introduction to the special study of astronomy, but rather for the student of mathematics who desires an insight into the creations of his masters in this field. The lack of a text-book, giving, within moderate limits and in a strictly scientific manner, the principles of mathematical astronomy in their present remarkably simple and lucid form, is undoubredly the reason why so many mathematicians extend their knowledge of the solar system but little beyond Kepler's law. The author has endeavored to meet this need, and at the same time to produce a book which shall be so near the present state of the science as to include recent investigations and to indicate unsettled questions.

FORD.-The Cranial Nerves. I2 pairs. By C. L. Ford, M.D., late Professor of Anatomy and Physiology in University of Michigan. Chart, 25 cents. 
FORD.-Classification of the Most Important Muscles of the Human Bady, With Qrigin Insertion, Nervous Supply and Principal Action of Each. By C. L. Ford, M.D., Iate Professor of Anatomy and Physiology in the University of Michigan. Chart, 50 cents.

FRANCOIS.-Les Aventures Du Dernier Abencerage Par Chateanbriand, Edited with Notes and Vocabulary. By Victor E. Francois, Instructor in French in the University of Michigan. Pamphlet, 35 cents.

GRAY.-Outline of Anatomy. A Guide to the Dissection of the Human Body, Based on Gray's Anatomy. 54 pages. Boards, 60 cents.

The objects of the outline are to inform the students what structures are found in each region and where the description of each structure is found in Gray's Anatomy. -Thirteenth edition, dated 1897 .

GREENE. - The Action of Materials Under Stress, or Structural Mechanics. With examples and problems. By Charles E. Greene, A.M., M.E., Professor of Civil Engineering in the University of

Michigan. Consulting Engineer. Octavo. Cloth, \$3.00.

Contents.-Action of a Piece under Direct Force. Materials. Beams. Tor sion. Moments of Inertia. Flexure and Deflection of Simple Beams. Restrained Beams: Continuous Beams. Pieces under Tension. Compression Pieces:-Columns, Pcsts and Struts. Safe Working Stresses, Internal Stress: Change of Form. Rivets: Pins. Envelopes: Boilers, Pipes, Dome. Plate Girder, Earth Pressure: Retaining Wall: Springs: Plates. Details in Wood and Iron.

HERDMAN-NAGLER.-A Laboratory Manual of Electrotherapentics. By IVilliam James Herdman, Ph.B., M.D., Professor of Diseases of the Nervous System and Electrotherapeutics, University of Michigan, and Frank IV. Nagler, B.S., Instructor in Electrotherapeutics, University of Michigan. Octavo. Cloth. I63 pages. 55 illustrations. $\$ 1.50$.

It has been our experience that the knowledge required by the student of medi cine concerning electricity and its relation to animal economy is best acquired by the laboratory method. By that method of instruction each principle is impressed upon the inind through several separate paths of the sense perception and a manual dexterity is acquired which is essential to success in the therapeutic applications.

This has been the plan adopted for teaching electrotherapentics at the University of Michigan. Every form of electric modality that has any distinctive physiological or therapeutical effect is studied in the laboratory as to its methods of generation, control and application to the pattent. We believe this to be the only practicable way for imparting the kind of instruction required for the practice of electrotherapeutics, but in our attempt to develop a naturally progressive and at the same time complete and consistent course of laboratory instriction we have found it a thing of slow growih.

This laboratory manual is the final result of our various trials and experiences, and while we do not claim for it either perfection in the arrangement of matter or completeness in detail, we feel that the time lias come for putting our plans in a form that will permin for it a wider usefulness as well as gain for it in the intelligent criticism of the experienced workers to the field which it seeks to cullivate.-From Preface.

HOWELL.-Directions for Laboritory Work in Physiology for the Use of Medical Classes. By W. H. Howell, Th.D., M.D., Professor of l'hysiology and Histology. Pamphlet. 62 pages. 65 cents.

HUBER.-Directions for Work in the Ilistolosical Laboratory. By G. Carl Huber. MI.D., Assistant Professor of Histology and Embryology, University of Michigan. Second,edition, revised and enlarged. Octavo. I9I pages. Cloth, \$1.50.

It is adapted for classes in medical schools and elsewhere where it is desired to furnish the class with material already prepared for the demonstration of strucure rather than to give instructiothin the technigue of the laboratory Provision for the 
latter is inade, however, by the addition of a section of about 50 pages on the methods for laboratory work. This section includes methods of macerating, hardening and fixing, decalcifying, impregnation, injecting, embedding, c:aining, and methods for preparing and staining blood preparations. The last is accompanied by an excellent plate of blcod elements. The selection of methods has in the main been judicious. The expositions are both clear and concise.-Journal of Comparative Neurologis.

In this little book Dr. Huher has given ns a model manual of microscopical technique in the laboratory study of histology. The subject matter is divided into convenient chapters, commencing with the cell and cell division (karyokinesis) in plant and animal life, and gradually developing, by easy stages, the most complex tissues of the animal and vegetable organism. Between each lesson blank pages are interleaved, to be used by the student for drawing the objects seen by him with a pencil or crayon-a most excellent plan as nothing fixes the appearance and characteristics of objects more firmly on the mind than drawing them, either free-hand or with a camera lucida (the former being preferable, as it educates the hand and eye). With each subject is given the source and origin, the best methods for obtaining and preparing it, and attention is called to the most noteworthy or characteristic points for examination.

The second part of the book is devoted to methods for laboratory work: soften ing, hardening, decalcification, etc., of the matter in gross; embedding, sectioning, staining and mounting. erc. The best stains, with methods of preparing the same, and, in short, a general formulary for the various reagents, etc., concludes the work, which is intended, as stated, as an aide meinoire supplementary to a course of lectures on histology.

We congratulate Dr. Huber on the skill with which he has developed the idea, and the didactic methods which he has employed. Such a book cannot but prove a great help to both student and teacher, and it should he more widely known.-St. Louis Medical and Surgeon's Juurnal.

JOHNSON.-Elements of the Law of Negotiable Contracts. By E. F. Johnson, B.S., LL.M., Professor of Law in the Department of Law of the University of Michigan. Svo., 735 pages. Full law sheep binding. $\$ 3.75$.

Several years of experience as an instructor has taught the anthor that the best method of impressing a principle upon the mind of the student is to show him a practical application of it. To remember abstract propositions, without knowing their application, is indeed.difficnlt for the average student. But when the primary principle is once associated in his mind with particular facts illustrating its application. it is more easily retained and more rapidly applied to analo ons cases.

It is deemed advisable that the student in the law sh ould be required, during his course, to waster in connection with each general branch of the law, a few well-selected cases which are illustrative of the philosophy of that subject. To require each student to do this in the larger law schools has been found to be impracticable, owing to a lack of a sufficient number of copies of individual cases. The only solution of this difficulty seems to be to plave in the hands of each student a volune containing the desired cases. In the table of cases will be found many leading cases printed in black type.-From Preface.

\section{LEVI-FRANCOIS.-A French Reader for Beginners, with Notes ana} Vocabulary. By Moritz Levi, Assistant Professor of French, University of Michigan, and Victor E. Fiancois, Instructor in French, University of Michigan. $12 \mathrm{mo}$. $26 \mathrm{I}$ pages. \$1.00.

This reader differs from its numerous predecessors in several respects. First, being aware that students and teachers in the French as well as in the German departm? nts of high schools and colleges are becoming tired of transiating over and over again the same old fairy tales, the editors have avoided them and selected some interesting and easy shor stories. They have also suppressed th 2 poetic selections which are never translated in the ciass room. Finally, they have exercised the greatest care in the gradition of the passages chosen and in the preparation of the vocabulary, every French word being followed not only by its primitive or ordinary meaning, but also by the different English equivalents which the text requires. Afrer careful examination, we consider this reader as one of the best on the American market.

LYMAN-HALL-GODDARD.-Algebra. By Elmer A. Lyman, A.B., Edwin C. Goddard, Ph.B., and Arthur G. Hall, B.S., Instructor in Mathematics, University of Michigan. Octavo. 75 pages. Cloth, go cents. 
MATTHEWS.-Syllabus of Lectures on Pharmacology and Therapemtics in the University of Michigan. Arranged Especially for the Use of the Classes Taking the Work in Pharmacology and Therapentics at the University of Michigan. By S. A. Matthews, M.D., Assistant in Pharmacy and Thorapeutice, University of Michigan. I $2 \mathrm{mo}$. II 4 pages. \$I.00.

MEADER.-Chronological Ontline of Roman Literature. By C. L. Meadér, A.B., lnstructor in Latin in University of Michigan. Chart, 25 cents.

MICHIGAN BOOK.-The U. of $M$. Book. A Record of Student Life and Student Organizations in the University of Michiran. Articles contributed by members of the Faculty and by prominent Alumni. $\$ 1.50$.

MONTGOMERY-SMITH.-Laboratory Manual of Elementary Chenistry. By Jabez Montgomery, Ph.D., Professor of Natural Science, Ann Arbor High School, and Roy B. Smith, Assistant Professor in Chemical Laboratory, Ann Arbor High School. I2 mo. 150 pages. Cloth, $\$ \mathrm{I} .00$.

This Work is intended as a laboratory guide to be used in connection with a good text-book or course of lectures, and in its arrangement and scope it is based upon the practical experience of two instructors in the Ann Arbor High School. It is therefore restricted to snch work as may be done by the average high school pupil. The experiments which are dis ected are given more to enable the student to comprehend the methods of analytical chemistry than to acquire particular proficiency in the work of chemical analysis. The work is characterized by minuteness of explanation, a feature which will be appreciated by the beginner.-Pharmaceutical tra.

NETTO.-The Theory of Substitutions and its Application to Algebra. By Dr. Eugene Netto, Professor of Mathematics in the University of Giessen. Revised by the author and translated with his permission, by F. N. Cole, Ph.D., formerly Assistant Professor of Mathematics in the University of Michigan, Professor of Mathematics, Colnmbia University. 8 vo. 301 pages. Cloth. \$3.00.

NOVY.-Laboratory Work in Physiological Chemistry. By Frederick (i. Novy, Sc.D., M.D., Junior Professor of Hygiene and Physiological Chemistry, University of Michigan. Second edition, revised and enlarged. With frontispiece and 24 illustrations. Octavo. Cloth, $\$ 2.00$.

This took is designed for directing laboratory work of medical students, and in showing them how to study the physics and physiology of the digestive functions of the blbod, the urine and other substances which the body contains normally, or which it specdily eliminates as effete material. The second edition has appeared within a very short time after the publication of the first. The firsi chapters deal with the facts, the carbohydrates and proteids. Then follow others upon the saliva, the gastric juice, the pancreatic secretion. the bile, blood, milk, and urine, while the closing cliapter deals with a list of reagents.

While the book is manifestly desigired for the use of Dr. Novy's own students. we doube not that other teachers will find it a valuable aid in their work. At the close of the volume are a number of illustrations of the various sedimentary substances found in the urine, taken from the work of voll Jaksch.--The Therapeutic uazelte

This book, although now in its second edition, is practically unknown to British readers. Up to the present, anyone wishing to find out liow a particular analytical metlod in physiological chemistry ought to be carried out, had of necessity to refer to a German text-book. This comparatively small book-for it only covers some three hundred pages-gives as good a generdi account of ordinary laboratory methods as any teacher or student could desire. Alshough the author refers in his preface to lielp derived from the works of Salkowski, Ilammasten and others, it is but Iair to say that the bonk lias undoubtedly becn written by one who has worked out the metlinds and knows the importance of exact practical details-Edinthurgh Med. Jurur., Scotland. 
Physiological chemistry is one of the most important studies of the medical curriculum. The cultivation of this field has until recently been possible to but few. The rapid development of this department of science within a few years past has thrown much and needed light upon physiological processes. It is from this quarter and from bacteriological investigations that progress must chiefly be expected. The rapid growth of this branch of chemistry is attended by another result. It necessitates the frequent revision of text-books. The present edition of Dr. Novy's valuable book is almost wholly rewritten. It is representative of the present state of knowledge and is replete with information of value alike to student and practitioner. Few are better prepared to write sucl a book than Dr. Novy, who has himself done much original work in this field.-The Medical Bullelin, Philad+lphia.

This is a greatly enlarged edition of Dr. Novy's work on Physiological Chemistry, and contains a large amount of new material not found in the former edition. It is designed as a text-book and guide for students in experimental work in the laboratory, and does not therefore cover the same ground as the works of Gamgee, Lea, and other authors of books on physiological chemistry. As a laboratory guide it should be adopted by our merlical colleges throughout the country, because it is an American production, contains only such directions and descriptions as have been verified by actual practice with students, and because it is clear, concise and definite in all its statements. Its tirst ten chapters treat of fats, carbohydrates, proteins, saliva, gastric juice. pancreatic secretion, bile, blood, milk, and urine. Chapter xi. is devoted to the quantitative analysis of urine, milk, gastric juice, and blood, while chapter xii. gives tables for examination of urine and a list of reagents.- $A m$. Medico-Surgical Bulleton, N.Y.

NOVY.--Laboratory Work in Bacteriology. By Frederick G. Novy, Sc. I)., M.D., Junior Professor of Hygiene and Physiological Chemistry, University of Michigan. Second edition, entirely rewritten and enlarged, 563 pages. Octavo. \$3.00.

As a teacher of bacteriology, the author has had extensive experience, and the second edition of his book will be highly prized by students for its practical service and thoroughness. The methods of investigation described are inainly those which have been employed in the hygienic laboratory or the University of Mlichigan, and they have stood the test of practical demonstration and usefulness. One of the mo: $t$ interesting parts of the book is the chapter on the chemistry of bacteria, and the general reader cannot fail to obtain from it a clear understanding of the con plex changes induced by these minute urganisms. The functions of the various ferments are also very cleverly discussed. An enumeration of the chapter headings will serve to show the scope of the work: Form and Classification of Bacteria; Size and Structure of Bacterial Cell; Life Hiscory of Bacteria; Environment of Bacteria; Chemistry of Bacteria; the Microscope; Cultivation of Bacteria; Non-Pathogenic Kacıeria; Bouillon, Agar, Milk and Modified Media, the Incubator and Accessories Relation of Bacteria to Disease-Methods of Infection and Examination; Pathogenic Bacteria; Yeasts, Moulds and Streptotrices; Examination of Water, Soil and Air; Special Methods of Work. To the latter subject, two chapters are devoted in which are very fully ontlined various special methods of value to advanced students.-Pharmaceutical Era, N. Y.

STRUMPELL.-Short Guide for the Clinical Examination of Patients. Compiled for the Practical Students of the Clinic, by Professor Dr. Adolf Strümpell, Director of the Medical Clinic in Erlangen. Translated by permission from the third German edition, by Jos. L. Abt. Cloth, 39 pages, 35 cents.

Preface to the Second Edition.-The second edition of this book has been improved by me in several parts, and particularly the sections treating of the examination of the stomach and nervous system nave bees slightly extended. The author trusts that the book may also fulfill its purpose in the future. in assisting the student to learn a systematic examination of the patient, and to impress on him the most importaut requisite means and methods.

SUNDERLAND.-One Uproard Look Each Day. Poens of Hope and Faith. Selected by J. T. Sunderland. Third Edition, I6 mo. White Binding, 30 cents; Cloth, 40 cents; Full morocco, 75 cents.

SUNDERLAND-Grains of Gold. Some Thoughts and a Brief Prayer For Each Day of the Months. Designed as Daily Helps in the Higher Life. Compiled by J. T. Sunderland. White Binding, 35 cents. 
WARTHIN.--Practical Pathology for Students and Physicians. A Mamual of Laboratory ana' Post-Mortem Technic, Designed Especially for the Use of Junior and Senior Students in Puthology at the Uniaersity of Michigan. By Aldred Scott Warthin, Ph. D., M. D., Instructor in Pathology, University of Michigan. Octavo. 234 pages. Cloth: \$I.5O.

We have carefully examined this book, and our advice to every student and practitiooer of medicine is-buy it. You will never regret having invested your money in it, and you will acquire such a large fund of information that the study of patloology will becone a pleasure instead of the drudgery which it sc unfortunately seems to be in many cases.

Part I. of this book, embracing some ${ }_{0} 3$ pages, deals with the materials, which includes the proper examination and notation of the gross changes which have occurred in every part of the body. In fact it is a complete exposé of what a com plete and accurate autopsy should be, the observance of which is oftener followed in the breach than in the actuality. Part Il., which includes 134 pages, deals with the treatment of the material. This is a very important part of tlue work, as it gives explicit directions in regard to the instruments to use, stains and staining methods, drawing, the preservation of specimens, nardening methods, in fact, of all those technical points connected with practical pathological microscopy. The examination of fresh specimens, injections, methods fixing specimens as well as special staining methods are taken up. In fact, space forbids us to give the entire, which are most valuable in every detail.--St. Louix Medical and surgical Journal.

WATSON. - Tables for the Calculation of Simple or Compound Interest and Discount and the Averaging of Accounts. The Values of Ammities, Leases, Interest in Estates and the Accumulations and Values of Investments at Simple or Compound Interest for all Rates and Periods; also Tables for the Conversion of Securities and Value of Stocks and Bonds. With full Explanation for Use. By James C. Watson, Ph.D., LI..D. Quarto. Cloth, \$2.50.

A book most valuable to bankers, brokers, trustees, guardians, judges, lawyers, accountants, and all concerned in the computation of interest, the division and settlement of estates, the negotiation of securities, or the borrowing and lending of inoney, is the above work of the late Professor James C. Watson, formerly Director of the Observatories and Professor of Astronomy at the Universities of Michigan and Wisconsin, and Actuary of the Michigan Mutual Life Insurance Company.

It contains, in addition to the usual tables for the calculation of simple or compound interest and discount, many tables of remarkable value, not found elsewhere, for the averaging of accoutns, the values of annuities, leases, interests in estates, and the accumulations and values of investments; also tables for the conversion of securities, and the values of stocks and bonds.

There are also given very full and clear explanations of the principles involved in financial transactions, and a great variety of miscellaneous examples are worked out in detail to illustrate the problems arising in interest, discount, partial payments, averaging of accounts, present values, annuities of different kinds, annual payments for a future expectation (as in life insurance), or for a sinking fund, conversion of securities, valucs of stocks and bonds, and life interests.

This book was issued from the press under the author's careful supervision. Professor Watson was noted for his clear insight into problems involving computations, and also for his wonderful ability in presenting the method of solution of such problems in a plain and simple manner. The varied array of practical examples given in connect:on with his "Table" shows these facts in a"remarkable manner. This book provides, for those least expert in calculations, the means of avoiding mistakes likely to occur; and for the man engrossed in the cares of business, the means of making for himself, with entire accuracy, the calculation which he may need, at the inoment when it is needed.

WRENTMORE-GOULDING.-A Text-Book of Elementary Mechanical Drazing for Use in Opice or School. By Clarence G. Wrentmore, B.S., C.E., and 1 Icrbert J. Goulding, B.S., M.E., Instructors in Descriptive Geometry and Drawing at the University of Michigan. Quarto. IO9 pages and I65 cuts. \$I.OO.

Tlis book is intended for a beginuers course in Elementary Mechanical Drawing for the office and scliool. Illustrations liave not been spared, and the explanations bave been made in a clear and concisc manner for the purpose of bringing the stu- 
othe desired results by the shortest route consictent with the imparting of an accurate knowledge of the subject.

The first chapter is devoted to Materials and Instruments; the second chapter, Mechanical Construction: third chapter, Penciling. Inking, Tinting; fourth chapter, Linear Perspective; fifth chapter, Teeth of Grass.

WRENTMORE.-Plain Alphabets for Office and School. Selected by C. G. Wrentmore, B.S., C.E., Instructor in Descriptive Geometry and Drawing, University of Michigan. Oblong. Ig plates. Half leather, 75 cents.

Souvenir of the University of Michigan, Ann Arbor. Containing 38 photo-gravures of President James B. Angell, prom.nent University Buildings, Fraternity Houses, Churches, Views of Ann Ar'bor, Etc., Etc. Done up in blue silk cloth binding. Price, 50 cents, postpaid.

Physical Laboratory Note Book.-A Note Book for the Physical Laboratory. Designed to be used in connection with Chute's Physical Laboratory Manual. Contains full directions for keeping a Physical Laboratory Note Book. II2 pages of excellent writing paper, ruled in cross sections, Metric System, size $7 \times 9 \frac{1}{2}$ inches. Bound in full canvass, leather corners. Price, by mail, 30 cents. Special prices to Schools furnished on application.

Botanical Laboratory Note Book.-A Note Book for the Botanical Laboratory. Contains directions for Botanical Laboratory. 200 pages of best writing paper, ruled with top margins. Pocket on inside of front cover for drawing cards. Bound in substantial cloth cover and leather back. Size $6 \times 91 / 2$. Price, by mail, 35 cents. Special prices to schools furnished on application. 




QP519

N85

Iovy, F.G.

Laboratory work in physiological

chemistry. 


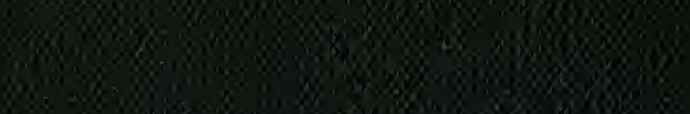

8.5.

$\frac{1}{2} \times \frac{2}{2}$ 